VALUES AND THEIR RELATIONSHIP TO PRO-ENVIRONMENTAL

ENGAGEMENT: A COMPREHENSIVE AND SYSTEMATIC INVESTIGATION

\author{
By Pollyane Kahelen da Costa Diniz
}

\author{
A thesis \\ submitted to the Victoria University of Wellington \\ in fulfilment of the \\ requirements for the degree of \\ Doctor of Philosophy \\ in Psychology
}

Victoria University of Wellington

2016 

In memory of my younger brother Pedro da Costa Diniz $(16 / 05 / 1987$ to $25 / 08 / 2012)$ 

Environmental problems are a result of maladaptive human behaviour. One way to tackle these problems is by fostering values that underlie pro-environmental engagement. Research has shown that self-transcendence values (e.g., social justice, equality, and world at peace) are positively correlated to environmental attitudes and behaviours. The present research aims to advance past research by systematically assessing the role of values in motivating individuals' pro-environmental engagement. Three empirical studies were conducted. Study 1 used a meta-analytical approach to provide a quantitative summary of research on the link between values and environmental outcomes. The final dataset included 47,660 participants from 41 countries and 90 independent samples. Results showed that the self-transcendence versus self-enhancement values dimension is the most often considered in the literature and, as expected, self-transcendence values are the best predictors of environmental outcomes. The analysis also showed that methodological aspects, such as the type of values measured, affected the association between values and environmental outcomes. Study 2 comprised two experiments using the value self-confrontation technique to promote value change and influence individuals' environmental behavioural intentions. Experiment 1 was conducted with 189 university students $(M=20.00, S D=3.43)$. Results from Experiment 1 showed that values were susceptible to change and that value change predicted environmental behavioural intentions. Experiment 2 was conducted with a sample of 115 participants from the general population $(M=35.00, S D=4.61)$ and partially replicated the findings of Experiment 1. Importantly, Experiment 2 found that value change was dependent on how strongly individuals felt connected to the reference group. Study 3 tested the moderation effect of moral identity, self-efficacy, self-control and consideration of future consequences in the link between values and environmental behavioural intentions. A total of 221 participants $(M=21.68 ; S D=5.92)$ took part in an online survey. Results indicated that only moral identity moderated the relationship between values and environmental behavioural intentions. It was found that moral identity enhanced the positive influence of selftranscendence values on environmental behavioural intentions. Findings are discussed considering the key role of self-transcendence values in fostering environmental behavioural intentions and the importance of identity in the values-environmental behavioural intentions link. Practical implications of the findings are also discussed.

Note: This thesis was a finalist for the Outstanding Postgraduate Research Award. 


\section{ACKNOWLEDGMENTS}

Kia Kaha

My $\mathrm{PhD}$ journey started even before moving to New Zealand when I was still doing the first year of my master's degree and working tirelessly in the lab, saving money for a flight overseas, volunteering in projects, building a CV and absorbing as much knowledge as I could at every single opportunity in my university in Brazil. All of that was with the aim of pursuing a dream: doing a $\mathrm{PhD}$ overseas. In the same month that I defended my master's thesis, I arrived in New Zealand. Getting through the $\mathrm{PhD}$ process was challenging but it was also full of good moments that brightened my personal "long walk to freedom". Many people have contributed in many ways for the completion of this goal and I am deeply grateful for their support.

Firstly, thanks to the participants of my empirical studies. Without them this thesis could not be done. Thanks also to all international scholars around the world who contributed data for my meta-analysis.

I am also very grateful to my PhD supervisors, Dr Taciano Milfont and Professor John McClure for agreeing to take me on and supervise this thesis. Thanks for their support and patience to read several drafts of chapters.

Thanks to Associate Professor Ronald Fischer for helping me advance my statistical knowledge, for believing in and supporting me, and for his mentorship and friendship. I deeply appreciate all the time you have invested and advice you have given to me. Thanks to Dr. Matt Crawford for the crucial feedback on independent studies composing this thesis.

Thanks to the examiners of this thesis Associate Professor Marc Wilson, Dr Nikki Harre and Dr Wesley Schultz for their expertise and constructive feedback.

I am also very grateful for the amazing work from all professional staff at Victoria University of Wellington, in particular, Al Abenoja, Alana Hamill, Barry Lewis, Crystal Li, Dalice Sim, Helen Gray, Kelly Atherton, Kerry Taplin, Kirsten Reid, Dr Lizzie Towl, Maria Goncalves-Rorke, Michael France, Ngaire Lavery, Patricia Stein, Professor Peter Whiteford, Shona de Sain, Sue D'ath, Sue O'Donnell, Tara Fischer, and Wendy Ward. Their understanding, humanity and efficiency were crucial in helping me to go through these past years. I will also be always grateful to Cathy Dole and Lynn Phillips for helping me to grow as a person. 
Thanks to Victoria University of Wellington for the financial support with the Victoria PhD Scholarship and the Submission Scholarship. I was awarded my very first scholarship in primary school for achieving the best grades, and after that continued to earn scholarships and awards for academic merit throughout my entire educational journey. Coming from a humble family I know the importance of having a scholarship to give high achievers the opportunity to strive for higher education when otherwise it would not be possible. I will always be grateful to those who make these scholarships available. Also thanks to the Faculty of Science, School of Psychology, Jacobs Foundation, and SASP for providing funding to attend conferences.

Thanks to every single member of the CACR. I could not ask for a better bunch of people to work, share life and travel with. Since the very beginning I felt welcomed and always found moral support here. Thanks to Morgan Davie, Rochelle Stewart-Allen and Maree Kibblewhite for been such helpful managers. Thanks to Dr Carla Crespo, Dr Diana Boer, Dr Katja Hanke, Dr Melanie Vauclair, Dr Sammy Khan and Dr Vivian Lung for the warm welcome and for bouncing back ideas. Thanks to Dr Aidan Tabor, Alana Cornforth, Alexis Garland, Alicia Bethel, Angela Robinson, Ben Tilyard, Camila Read, Carmen Leong, Dr Chris Low, Claudia Recker, Dr Clara Cantal, Dr Eunro Lee, Floor Spijkers, Dr Jessie Wilson, Laina Isler, Dr Mohsen Joshanloo, Dr Paula Speer, Dr Paul Reddish, Dr Penny Tok, Reneeta Mogan, Shachi Phadke and Tamara Khair for the companionship and baked goods. Thanks also to Dr Adrienne Girling for all the proofreading, advice and encouragement to continue. Your friendship and support were fundamental for the completion of this journey. Thanks for being by my side and for helping so much with everything. Thanks also to Dr Agnes Szabo for sharing all her research and statistical knowledge. Thanks to Dr Larissa Kus (Larissinha) for the wise advice and lovely friendship. Thanks to Dr Elena Maydell for checking formatting. Thanks to Dr Raquel Pérez for the 'anytime' online support. You are all the most precious things that came out of this experience. Thanks to Chelsea Rose for accommodating my tutor duties with my PhD work. Thanks to Štěpánka Dvořáková, Samantha Rae and Teressa Cho for helping with data-collection.

A big thank you to my best friend Ingrid Kennedy for being with me since the very first day I arrived in New Zealand until now; in happy and sad moments. Thanks for always believing in my potential and for the hugs too! 
Thanks to my Brazilian friends that, even from afar, continuously cheered and supported me: Luciana Chacón, Jane Palmeira, Camila Mendes, Jana Barros, Katharine Fontes, Laysa Silva Soares, Juliana Teixeira, Renata Athayde and Tyrza Lima.

Thanks to all my New Zealand friends, especially Liviu Sas, Ara Kim, Gillian Tito for always been there to help when I needed, share laughs and yummy dinners. Thanks to my Capoeira group for keeping me close to my country and offering moments of stressrelease and joy that kept me sane.

Thanks to my family for enduring with my absence. Thanks to my mum, dad, brothers (to my strong older brother and to my younger brother who tragically left us while I was doing this thesis; I am so grateful you shared your years on earth with me), niece and nephew for holding onto me, supporting me and bringing us all together. Especially thanks to my mum for the inspiring strength and for her sacrifices to protect her children.

Thanks to my partner Ryan McMillan for being always by my side, and putting up with "PhD Polly". Thanks for being understanding in all occasions, for believing and reassuring me that I will do it and above all thanks for your love and support. I would not have done it without you.

This thesis is not only an academic accomplishment but also a very important chapter in my life. Thanks to all of you close or afar that shared it with me. The $\mathrm{PhD}$ took me around the world to study and travel, enriched me intellectually, challenged my determination and externalised my fears. It gave me the opportunity to meet a lot of very interesting people from ego-maniacs to the golden-hearted, and to see many places I have never imagined I would before. It was a bumpy road, and just like life, uncontrollable events happened. On many occasions it felt unachievable, but having come out the other end I can reassure you that everything is possible. Everyone has their own journey and a little bit of hope, a lot of perseverance and a handful of true friends are your most powerful assets.

I had to overcome enduring hardship (financial, health and grief) to achieve my goals, but education is a very precious thing and I am very proud to be the first in my family to get a university degree. Along the years, many people told me I would not get that far, so I wanted to register to anyone with a big dream who feels like they don't have the means to achieve it that everything is possible and you can do whatever you put your mind and heart into. So never ever let anyone tell you otherwise and above all enjoy the ride. 


\section{TABLE OF CONTENTS}

Acknowledgements $\mathrm{V}$

Table of Contents . .......... ix

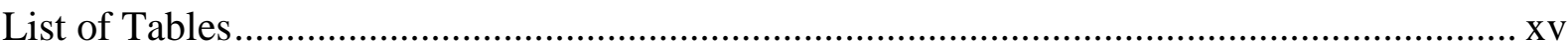

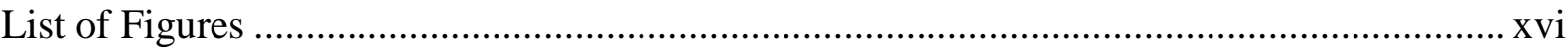

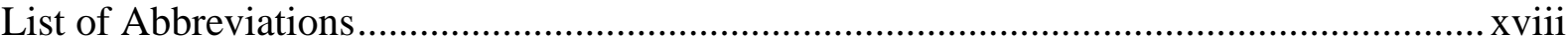

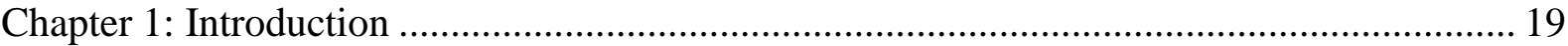

The Urgency of Knowing the Psychological Causes of (non) Ecological Acts - Why Are

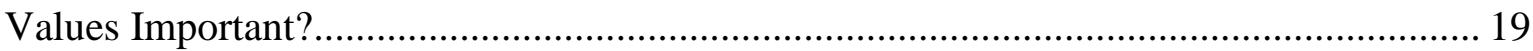

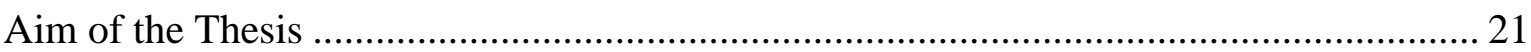

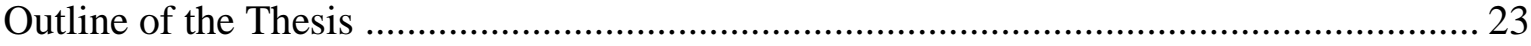

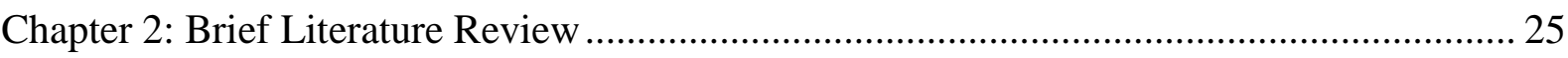

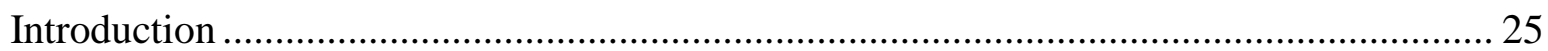

Section 1. Environmental Engagement: Conceptualisation and Measurement................... 25

Brief Overview of Environmental Psychology …....................................................... 25

Defining Environmental Engagement for the Context of This Thesis .......................... 26

Explaining the Relationships between Environmental Attitudes and Behaviours:

Contributions from the Theory of Planned Behaviour (TPB) and Identity Research .... 28

Measuring Environmental Engagement (Attitudes, Behaviours, Concern and

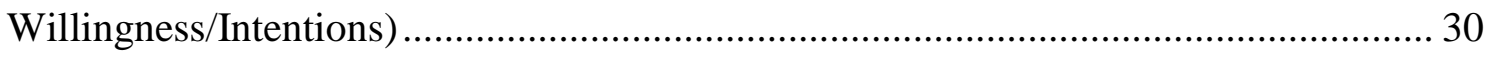

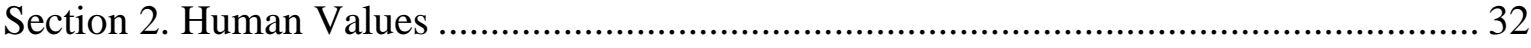

Theoretical Approaches on Values: Rokeach, Inglehart, Hofstede and Schwartz ......... 33

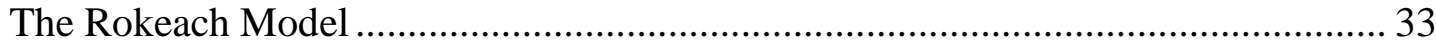

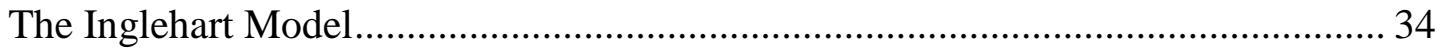

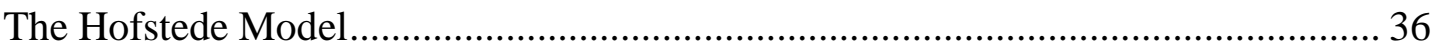

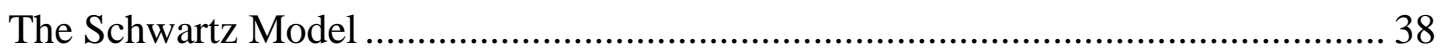

The Schwartz Value Survey (SVS) .................................................................. 41

Critiques and new developments on Schwartz's values theory and measure............. 43

Application of Schwartz's values theory and environmental engagement: Selftranscendence / self-enhancement values and environmental outcomes 


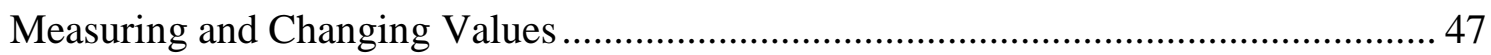

Development of a Model of Environmental Values ......................................................... 48

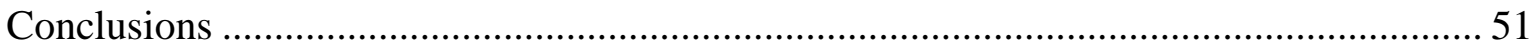

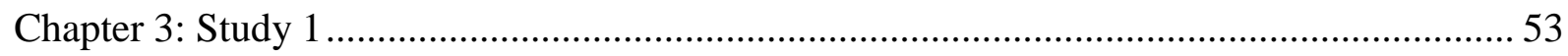

A Meta-analysis on the Relations between Values and Environmental Engagement ......... 53

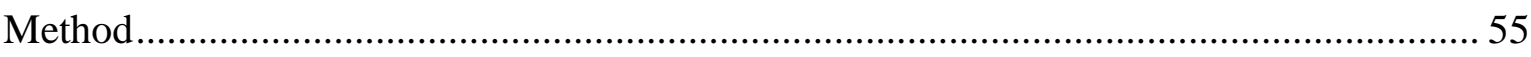

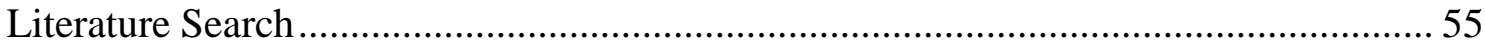

Inclusion-exclusion Criteria and Coding of Study Characteristics ................................ 55

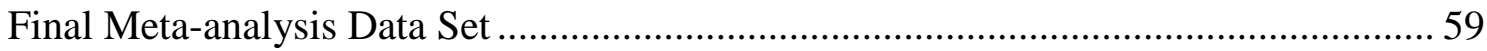

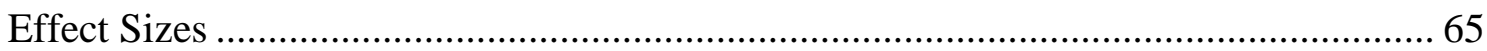

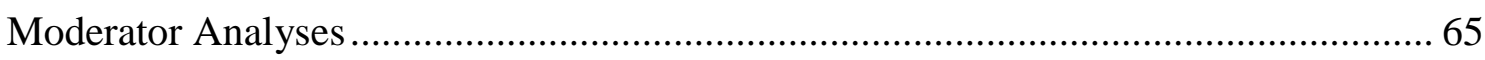

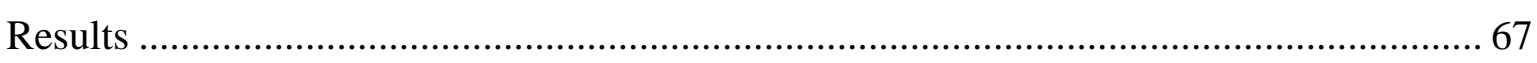

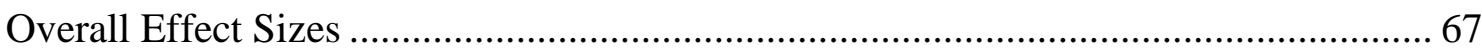

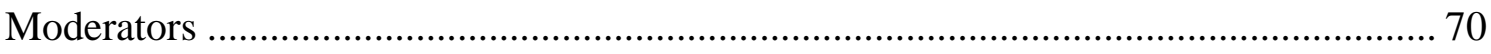

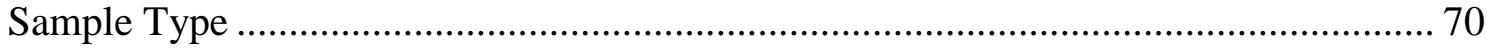

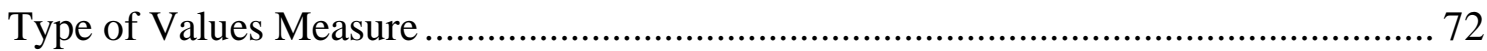

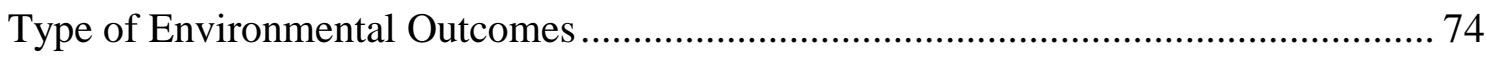

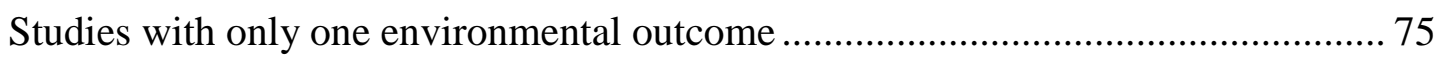

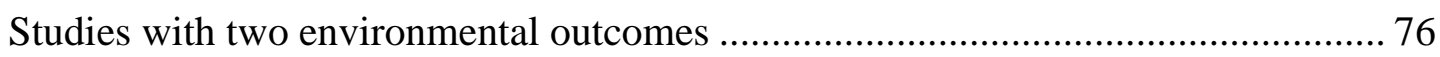

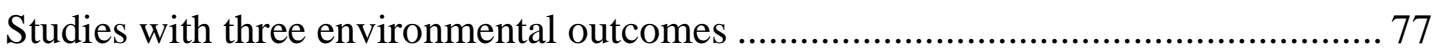

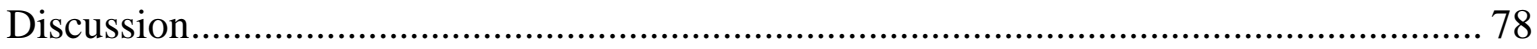

On the Associations between Values and Environmental Engagement ......................... 78

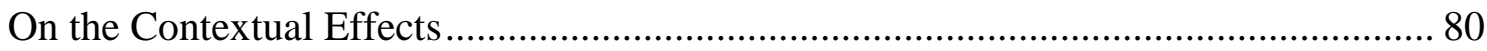

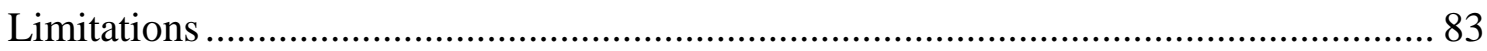

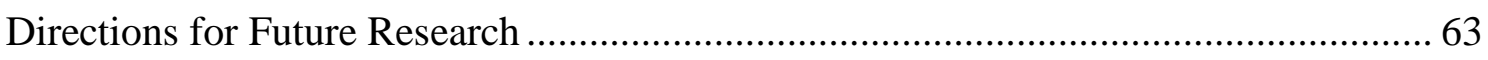

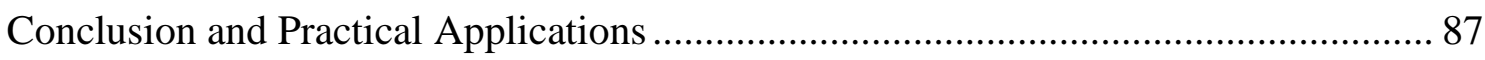

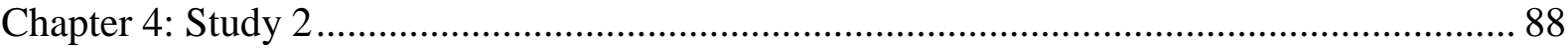

Experimentally Manipulating Values to Motivate Environmental Behavioural Intentions 88

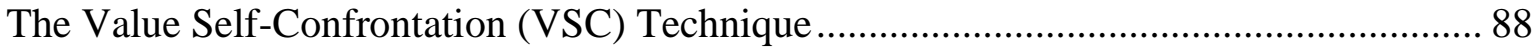

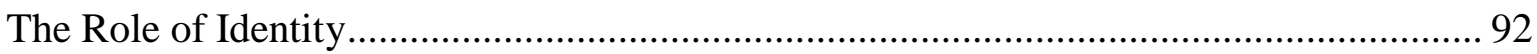

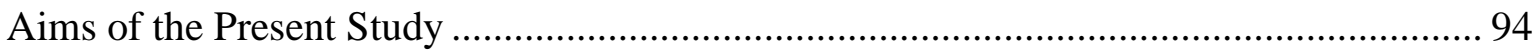

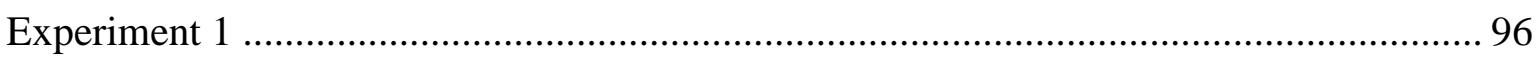

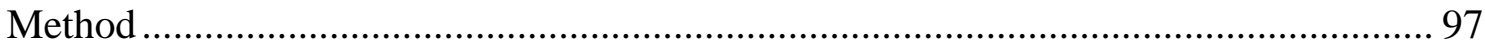




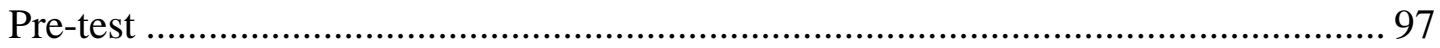

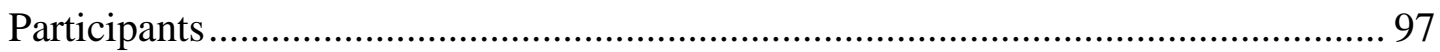

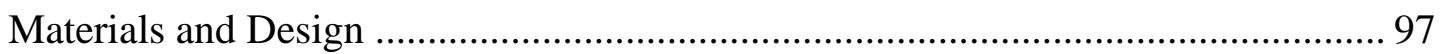

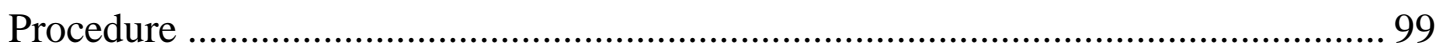

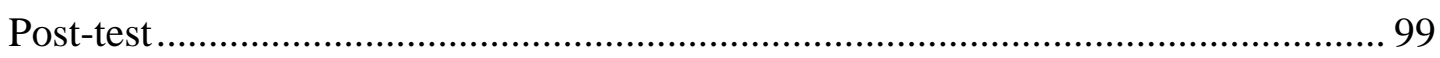

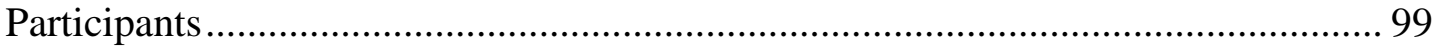

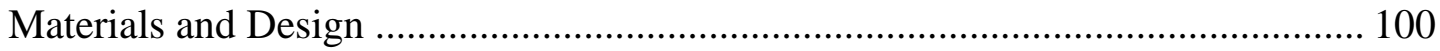

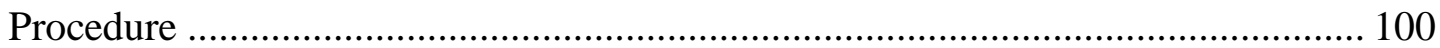

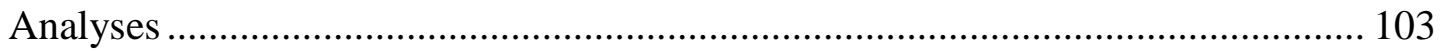

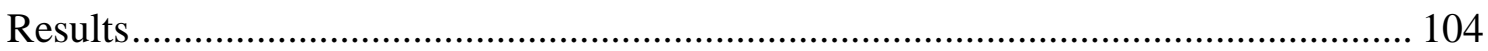

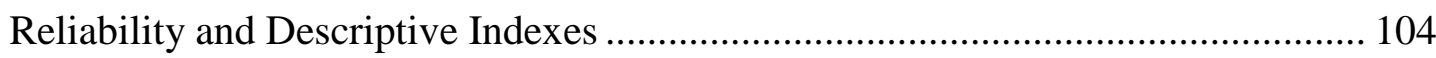

Time, Values and Condition ............................................................................. 104

Time, Condition and Student Identity .............................................................. 109

Change on Values and Environmental Behavioural Intentions .............................. 113

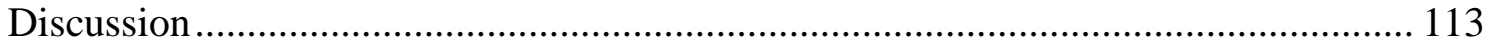

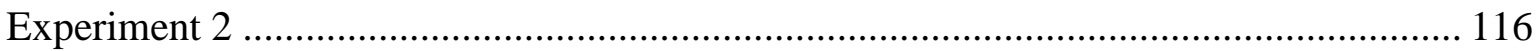

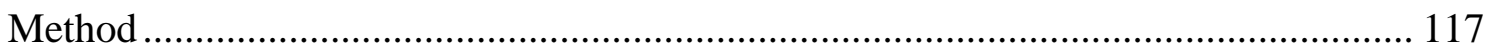

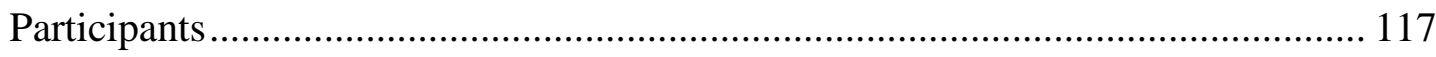

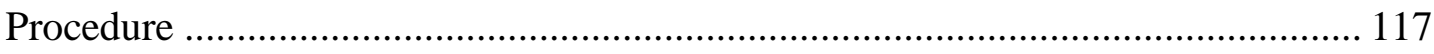

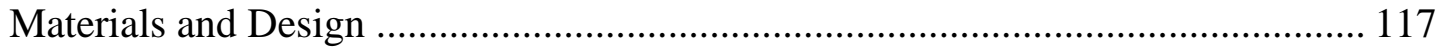

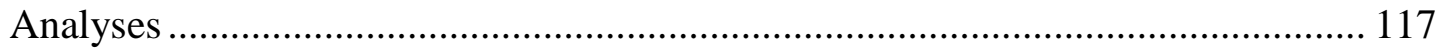

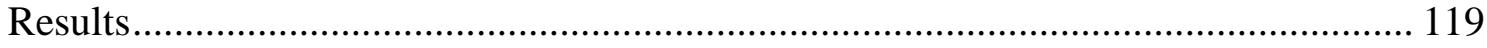

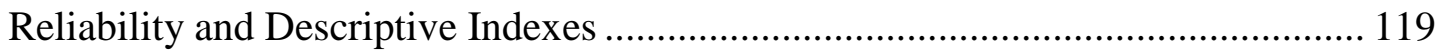

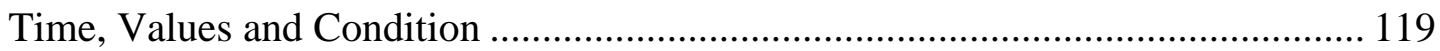

Time, Condition and National Identity .......................................................... 124

Change on Values and Environmental Behavioural Intentions ............................ 127

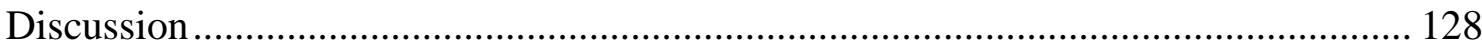

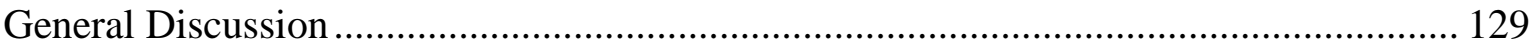

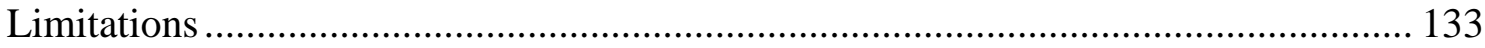

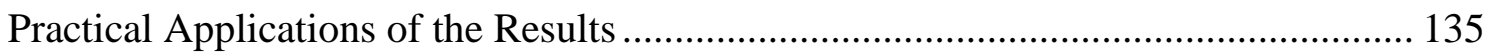

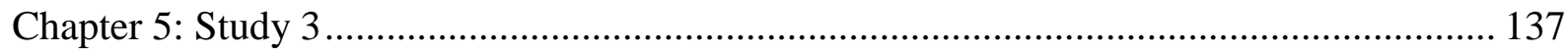

Testing the Moderator Effect of Individual Differences in the Relationship between Values

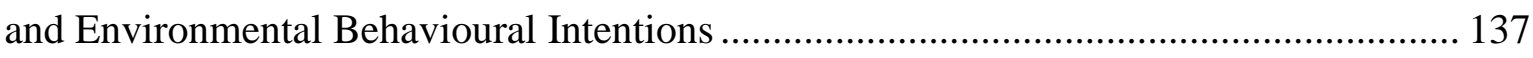


Why Moral Identity, Self-control, Self-efficacy and Consideration of Future

Consequences?

Moral Identity as Moderator of the Values-Environmental Behavioural Intentions

Link

Self-control as Moderator of the Values- Environmental Behavioural Intentions

Link

Self-efficacy as Moderator of the Values- Environmental Behavioural Intentions

Link

Consideration of Future Consequences as Moderator of the Values- Environmental

Behavioural Intentions Link

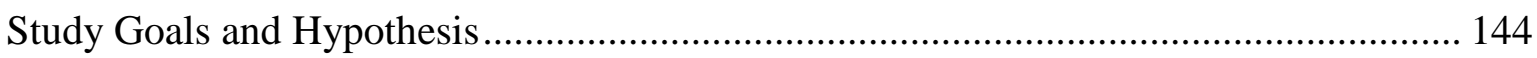

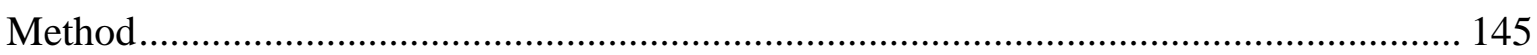

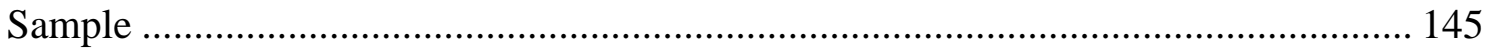

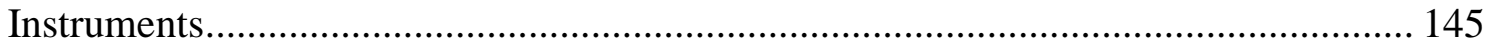

General Ecological Behaviour Scale (GEB) …..................................................... 145

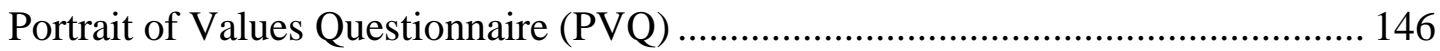

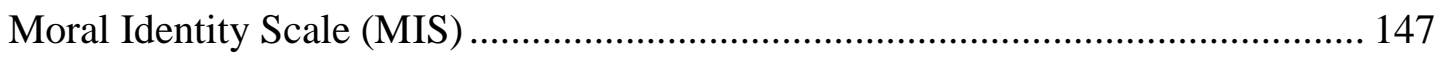

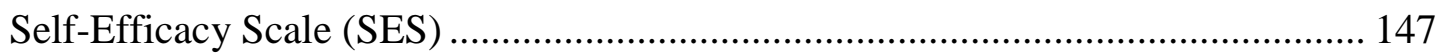

Brief Self-Control Scale (BSCS) ................................................................... 147

Consideration of Future Consequences (CFC) Scale ............................................ 147

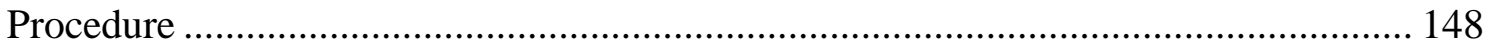

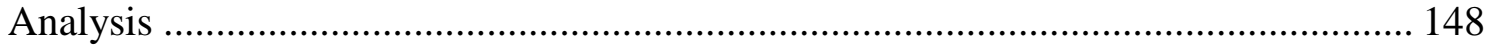

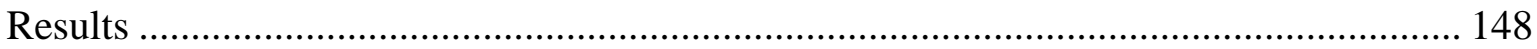

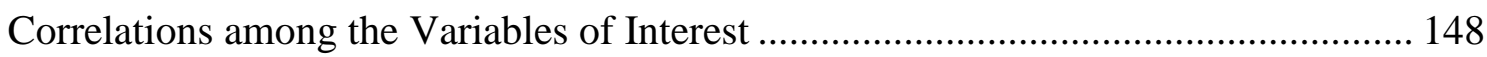

Relationships between Demographic Variables and the Dependent Measure ............. 151

Moderated Regression Analysis and Simple Slopes Calculations .......................... 151

Examining the Robustness of the Moral Identity Moderation ................................. 156

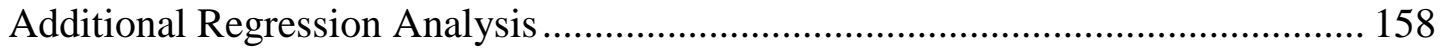

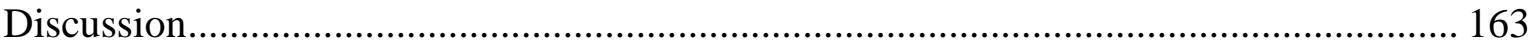

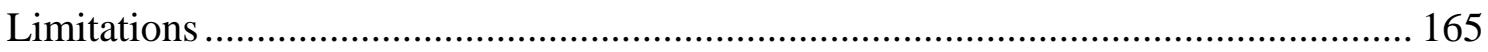

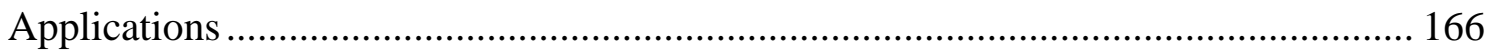

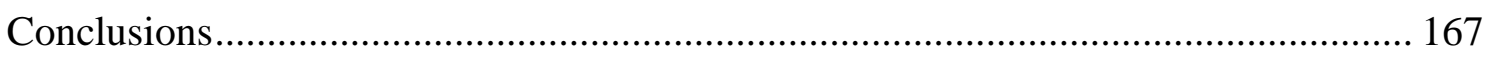

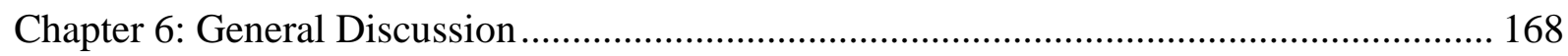


Overview 168

Key Research Findings

1. Self-transcendence Values are the Primary Values Predicting Environmental Outcomes

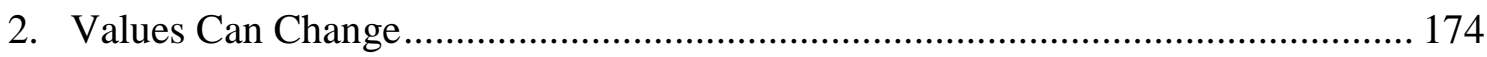

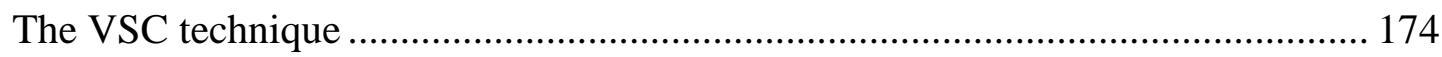

The prediction of environmental behavioural intentions ...................................... 176

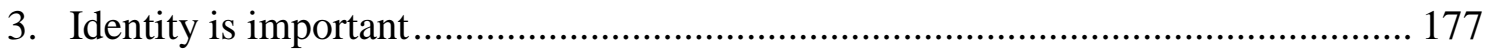

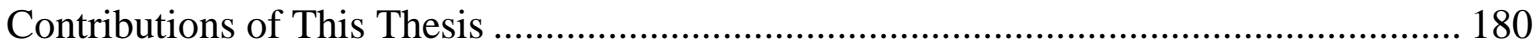

1. Theoretical Implications of the Current Thesis …................................................. 180

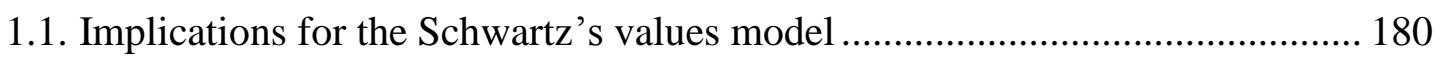

1.2. Implications for the use of the VSC technique: The underlying mechanism of value-change and the role of identity ........................................................... 182

2. Methodological Contributions of the Current Thesis ........................................... 184

3. Integrated Model of Values, Identity and Environmental Outcomes ..................... 186

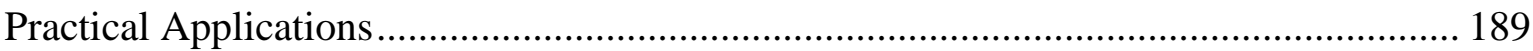

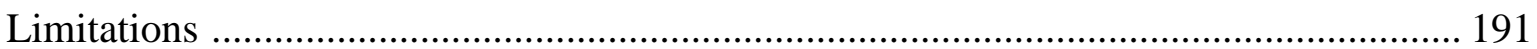

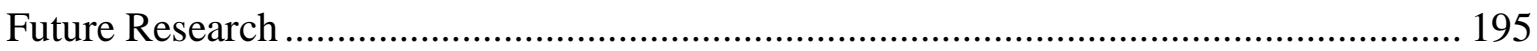

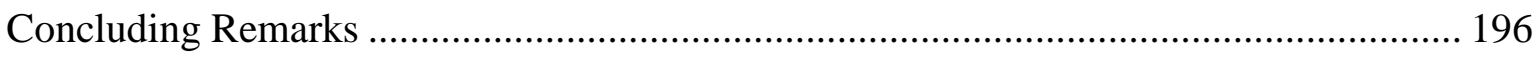

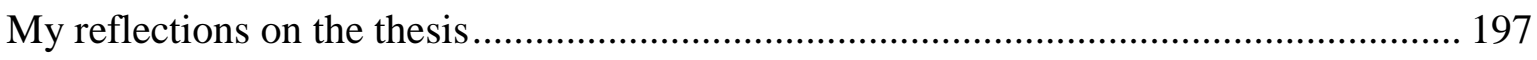

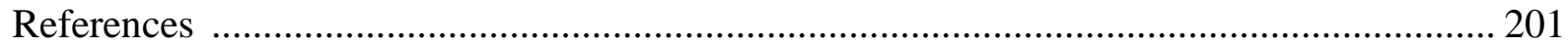

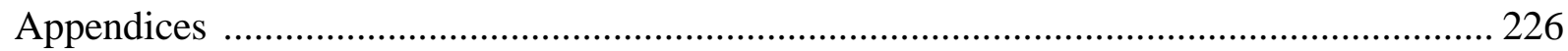

Appendix A. Additional Information on Adaptation of GEB Scale................................. 226

Appendix B. Questionnaire for Pilot Study - Adaptation of General Environmental

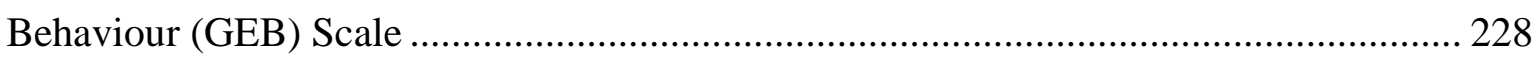

Appendix C. Information Sheet for Pre-test (mass testing) - Experiment 1 .................... 232

Appendix D. Questionnaire for Pre-test (mass testing) - Experiment 1 ........................... 234

Appendix E. Information Sheet for Post-test - Experiment 1 .......................................... 238

Appendix F. Self-transcendence Values Condition - Experiment 1................................ 240

Appendix G. Self-enhancement Values Condition - Experiment 1................................. 248

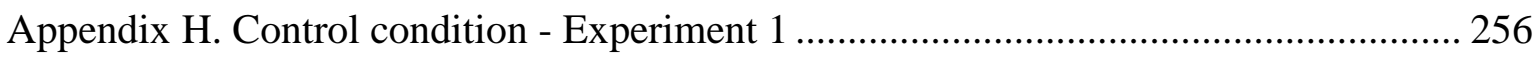

Appendix I. Email and Debriefing Sheet for Experiment 1 ......................................... 265

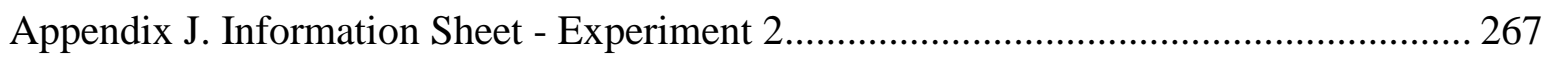


Appendix K. Self-transcendence Values Condition - Experiment 2 ............................... 268

Appendix L. Self-enhancement Values Condition - Experiment 2 ................................ 276

Appendix M. Control Condition - Experiment 2 …...................................................... 284

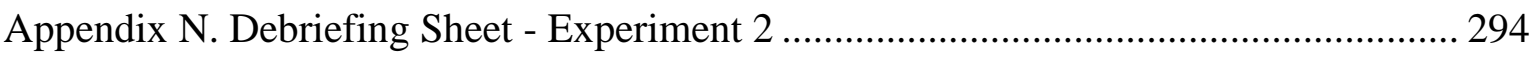

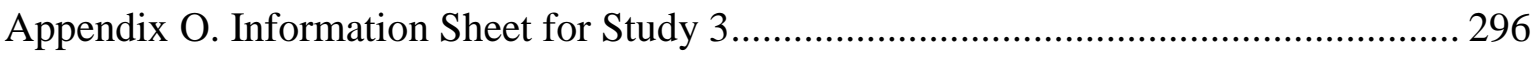

Appendix P. Measures Included in the Online Survey for Study 3 .................................. 298

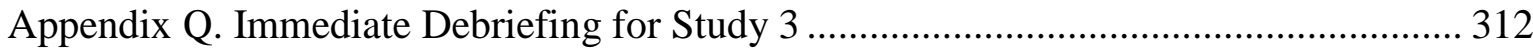




\section{LIST OF TABLES}

Table 3.1. Studies Included in the Meta-analysis and Coding Variables 60

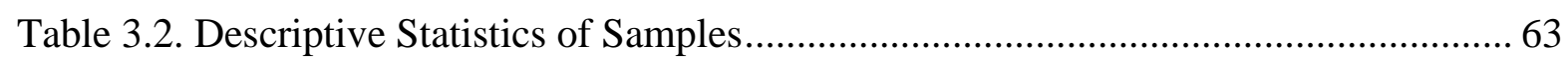

Table 3.3. Number of Samples and Subjects by Country and Region of the World ............. 64

Table 3.4. Summary of Effect Sizes for Values and Environmental Engagement ................ 68

Table 4.1. Reliability and Descriptive Indexes of Measures Used for Experiment 1......... 104

Table 4.2. Reliability and Descriptive Indexes of Measures Used for Experiment 2 ......... 119

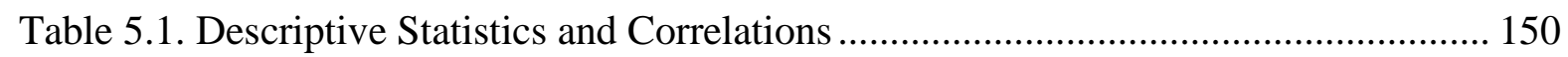

Table 5.2. Hierarchical Regression of the Prediction of Environmental Behavioural

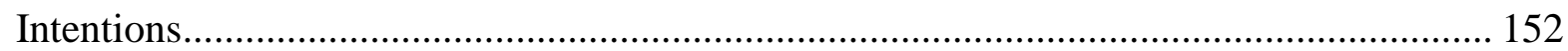

Table 5.3. Hierarchical Regression of the Prediction of Environmental Behavioural

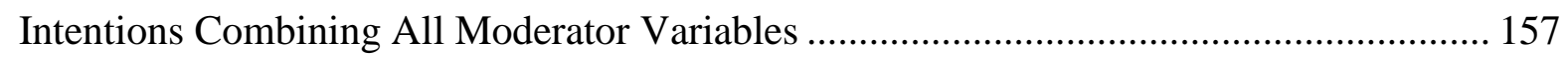

Table 5.4. Hierarchical Regression of the Prediction of Environmental Behavioural Intentions for Self-transcendence Values 159

Table 5.5. Hierarchical Regression of the Prediction of Environmental Behavioural Intentions for Self-enhancement Values 160 


\section{LIST OF FIGURES}

Figure 2.1. Circular model of relations among ten motivational value types at the individual level.

Figure 4.1. Changing some specific values increased the importance of values that served the same or similar motives and decreased the importance of values that served opposing motives

Figure 4.2. Priming specific values caused participants to exhibit more behaviour consistent with the primed values, while less behaviour consistent with the opposed values

Figure 4.3. Values scores according to time and type of values

Figure 4.4. Values scores according to type of condition (self-transcendence, selfenhancement and control conditions), type of values (self-transcendence and selfenhancement values) and time (pre-test and post-test)

Figure 4.5. Self-transcendence minus self-enhancement values scores according to time (pre-test and post-test) and type of condition (self-transcendence, self-enhancement and control conditions)

Figure 4.6. Self-transcendence minus self-enhancement values scores according to time (pre-test and post-test) and type of condition (self-transcendence, self-enhancement and control conditions) in low (A) and high identity score (B) groups

Figure 4.7. Values scores according to type of values (self-transcendence and selfenhancement values) and type of condition (self-transcendence, self-enhancement, and control conditions).

Figure 4.8. Values scores according to time (pre-test and post-test) and type of values (selftranscendence and self-enhancement values)

Figure 4.9. Values scores according to type of condition (self-transcendence, selfenhancement and control conditions), type of values (self-transcendence and selfenhancement values) and time (pre-test and post-test)

Figure 4.10. Self-transcendence minus self-enhancement values scores according to time (pre-test and post-test) and type of condition (self-transcendence, self-enhancement and control conditions) in low (A) and high identity score (B) groups

Figure 5.1. Model depicting the proposed conditional effect of values on environmental behavioural intentions moderated by moral identity, self-control, self-efficacy and consideration of future consequences 
Figure 5.2. The levels of moral identity enhanced the positive effects of 'pure' selftranscendence values scores on environmental behavioural intentions (moderators included separately in four different sets of analyses)

Figure 5.3. The levels of moral identity enhanced the positive effects of 'pure' selftranscendence values scores on environmental behavioural intentions (moderators included

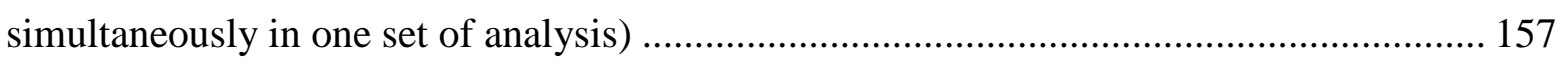

Figure 6.1. Schematic summary of main findings from each study ................................. 170

Figure 6.2. A comprehensive portrayal of values, identity and environmental engagement 188 


\section{LIST OF ABBREVIATIONS}

CFC Consideration of Future Consequences

PVQ Portrait of Values Questionnaire

SE Self-enhancement

ST Self-transcendence

SVS Schwartz Values Survey

TPB Theory of Planned Behaviour 


\section{CHAPTER ONE: INTRODUCTION}

\section{The Urgency of Knowing the Psychological Causes of (Non) Ecological Acts - Why Are Values Important?}

Research suggests that environmental problems, such as global warming and damaged water supplies, are a result of human actions that harm the natural environment (Maloney \& Ward, 1973; Schultz \& Zelezny, 1998). Many scholars point to environmental problems as the most significant current global issue and perhaps the greatest challenge to current civilisation (Bain, Hornsey, Bongiorno, \& Jeffries, 2012; Milfont \& Page, 2013; Spence, Leygue, Bedwell, O'Malley, 2014; Steg, Bolderdijk, Keizer, \& Perlaviciute, 2014; Zelezny \& Schultz, 2000). As a result, the issue has gained global attention. It is not by chance that environmental problems form a part of the United Nations Development Goals and international discussions on the topic (see, e.g., the Copenhagen Climate Change Conference 2009 and subsequent conferences in Cancun 2010 and Warsaw in 2013 by UNFCCC) are frequent and ongoing. The news media quite often issue reports and notices on the topic, bringing the need of reinforcing environmental concern to the attention of the global community. For example, National Geographic Magazine has a range of online publications focused on "Environment" which cover topics such as the energy challenge, struggles with freshwater resources, and global warming. Most recently a youtube.com video of 'Last week tonight with John Oliver' (2014, May 11), from the HBO TV program showcasing a debate between a well-known scientist, a comedian and a 'denier ${ }^{1}$ ' on the topic of climate change went 'viral' among social media users (week from 12-16 May, 2014).

While public debate continues, the Intergovernmental Panel on Climate Change designates human behaviour as the primary cause of global temperature increases since the mid-20 ${ }^{\text {th }}$ century (Pachauri \& Meyer, 2014; Pachauri \& Reisinger, 2007). Due to rising awareness of the consequences of human impact on the natural environment (Crompton, 2010; Zelezny \& Schultz, 2000), environmental, governmental and non-governmental organisations have been discussing the need for promoting beliefs, values, attitudes, and

\footnotetext{
${ }^{1}$ The term 'denier' refers to people who proclaim that global warming and climate change is nothing more than a hoax (Bain et al., 2012). Also called nonbelievers, they deny that climate change is a true concern.
} 
behaviours in regard to environmental sustainability (Crompton, 2010; Lawrence, Cornforth, \& Barrett, 2011; Manning, Reisinger, \& Wratt, 2009). Given the potential benefits that may be gained by understanding the role of values in this context, social scientists have been working to understand more about how humans relate to the environment, and have thus uncovered techniques that could be used to encourage people to live in a more sustainable manner (Gardner \& Stern, 2002; Hawcroft \& Milfont, 2010). For example, Barr (2007) states that the household waste problem is one that is likely to be resolved only when policies are implemented that are based on a clear understanding of what factors influence individual intentions and behaviours, which in turn need to be grounded in rigorous psychological and social research. Thus, the field of psychology can play an important role in reducing environmental problems by investigating the motivations and underlying determinants of human behaviour which affect the natural environment, and by understanding the formation of habits, and the process of socially learned pro-environmental behaviour. The focus on psychological mechanisms for reducing environmental problems combined with the knowledge from the natural sciences on the objective effects of harming the natural environment, can inform public policies aimed at reducing environmental problems.

Many countries have adopted a range of strategies to protect the environment involving public policies of large scale such as signing the Kyoto protocol or regulating people's daily behaviour by investing in campaigns to promote environmentally friendly acts (e.g., picking up rubbish from the beaches and participating in a well organised and efficient recycling system) (Crompton, 2010). However, it is still unclear why many people say they are environmentally friendly but do not act as if they are. For example, deciding to take a bath after a day's work instead of taking a quick shower when water supplies are limited, or cleaning the streets with running water in locations where communities are suffering from water restrictions, are evidence of the contradictions between intentions and actions. Unfortunately, the reasons that individuals act in environmentally unsustainable ways are still not well understood. For example, it has been assumed that social desirability motives influence how individuals respond when they are directly questioned about whether they preserve the environment (Beckman, 2005). However, work from Kaiser, Wölfing, and Fuhrer (1999) and Milfont (2009a) has shown that social desirability does not necessarily have a large direct effect on people's individual environmental behaviour. Therefore, it can be argued that there must be many more multidimensional psychological aspects at play to explain how humans engage with the environment. 
Hence there is substantial value in clarifying how psychological constructs influence an individual's environmental engagement. An important promising step towards achieving this goal is to study human values. Individuals apply values as core assumptions, which they use in making decisions in everyday life, such as recycling and saving water. In a report by the World Wide Fund for Nature (Crompton, 2010) there is a clear recognition that human values are important to understand people's behaviour towards the environment. According to the report, it is increasingly evident that resistance to action on global challenges, including campaigning against humanitarian and environmental crises, will only be overcome if we engage with the values underpinning this resistance (Crompton, 2010). Furthermore, research on values suggests that evidence-based programs aimed at promoting sustainability by changing behaviours through social influence are more effective than programs based on education (Schultz \& Kaiser, 2012; Schultz, Khazian, \& Zaleski, 2008). The research presented in this thesis will attempt to highlight and target values as one important factor that influences individuals' intentions and behaviours towards the environment.

\section{Aim of the Thesis}

This research endeavours to clarify the link between values and environmental engagement in a number of ways. First, although previous studies have examined the relationship between values and environmental engagement (for example, Milfont, Duckitt \& Wagner, 2010; Schultz et al., 2005; Stern, Dietz, Kalof, \& Guagnano, 1995), so far no study has attempted to quantitatively integrate previous findings on values and environmental outcomes (leading to Study 1).

Second, although there is an assumption (based on findings from survey-based research) that values predict environmental behaviour, no previous study has systematically assessed whether values can be manipulated to influence individuals' behavioural intentions towards the environment. According to the theory of reasoned action (Ajzen \& Fishbein, 1980) and its revised formulation, the theory of planned behaviour (TPB) (Ajzen, 1991), behavioural intention is the immediate antecedent of overt behaviour. That is, behavioural intentions are better predictors of actual behaviour than more distal constructs such as attitudes. Intentions, in turn, are affected by psychological constructs such as subjective norms, perceived control and attitudes. In line with the TPB, research has shown that values are related to intentions to engage in environmental behaviours (Axelrod, 1994). Given the link between intention and behaviour and the influence of values on intentions, as outlined 
above, there is importance in understanding how values can be experimentally manipulated in order to change people's priorities, and how this change may affect environmental behavioural intentions (leading to Study 2).

Furthermore, research has shown that identity, especially social identity, plays an important role in promoting values (Hitlin, 2003; Nakashima, Isobe, Souma, \& Ura, 2013; Terry, Hogg, \& White, 1999) and also in explaining intentions to engage in environmental acts (Fielding, McDonald, \& Louis, 2008). Studies have suggested that values change through norms (Milfont, Sibley, \& Duckitt, 2010; Oreg \& Katz-Gerro, 2006), and that people are more prone to conform to a social norm if they have a strong affiliation with their norm group (Berzonsky \& Sullivan, 1992; Marcia, 1966). Moreover, researchers have found that group identification and national identification relates to pro-environmental actions and that framing environmentalism as patriotic is an efficient strategy to diminish denial of environmental issues and promote more pro-environmental actions (Feygina, Jost, \& Goldsmith, 2010). These findings suggest that people with high levels of group identity can become more environmentally friendly. Therefore, Study 2 will also investigate the effect that group identity (i.e., university student) and national identity (i.e., New Zealander) exert on the relationship between value change and the promotion of environmental behavioural intentions.

Third, although the multinational research on environmentalism published to date has focused on the relationship between environmental attitudes, behaviours and values (e.g., Dunlap \& Mertig 1995; Schultz \& Zelezny, 1999), studies exploring the valuesenvironmental engagement link have so far neglected variables, such as individual characteristics, that could moderate this relationship. Clarifying moderating variables (e.g., moral identity, self-efficacy, self-control and consideration of future consequences-CFC) will enable researchers to understand how to strengthen the relationships between values and environmental engagement, and thereby promote environmental acts. An investigation of the moderating variables will shed light on the optimal conditions under which values can be used to evoke environmental outcomes (leading to Study 3 ).

In sum, this thesis will be one of the first endeavour to exam meta-analytically the main values theories that influence environmental engagement. It assesses changes in values using an experimental approach, while investigating the effect of this manipulation on behavioural intentions toward the environment. Furthermore, it also provides a novel attempt 
to investigate the effect of student and national identity and the moderating role of individual characteristics on the relationships between the two primary constructs of this thesis: values and environmental behavioural intentions.

Besides the potential theoretical contribution to the field, there are also practical applications of the present research. First, the findings will be able to provide robust researchbased knowledge that can be used to improve methods aimed to deploy our values in collective decision-making. Second, the present research has the potential to improve environmental behaviour change campaigns by applying a novel value change approach. In the past, such campaigns have almost exclusively relied on environmental education - a technique that is typically unsuccessful (see Schultz \& Kaiser, 2012). However, as Crompton (2010) points out, communicators would be able to have more effective campaigns by understanding the integrated nature of value systems and how their communications can activate (or strengthen) certain values that have a positive impact on people's environmental behaviours.

\section{Outline of the Thesis}

Chapter Two of this thesis consists of a literature review of theory and research on environmental engagement and human values. Section one of Chapter Two starts with a brief overview of environmental psychology followed by the definitions of the different concepts that together make up the term 'environmental engagement' (environmental attitudes, behaviours, concern, and willingness). Section one also presents a brief review of the different measures of environmental outcomes; specifically, it focuses on describing the most widely used questionnaires to assess these constructs, highlighting the flaws and the progress made in trying to accurately measure a diverse number of environmental outcomes. Section two consists of a review of the literature on values, specifically to provide a brief description of four theoretical approaches on values, Rokeach's model, Inglehart's model, Hofstede's model, and Schwartz's model. This section addresses how values are defined and presented in the relevant literature and how they are related to environmental engagement. The chapter also outlines the Schwartz's values theory as the theoretical framework used in the thesis providing a discussion about the main concepts proposed by the theory, the Schwartz Value Survey, some critiques and new developments in Schwartz's values theory and measure, with an emphasis on the Portrait Values Questionnaire. Chapter Two concludes by presenting the 
applications of Schwartz's values theory to explain environmental outcomes and discusses how to measure and change values.

Chapters Three, Four and Five of this thesis report three empirical studies. Chapter Three (Study 1) describes a meta-analysis, which tested the effect of values on environmental engagement by gathering and quantitatively summarising the available data on the topic. Chapter Four (Study 2) describes two experiments. In both experiments, values dimensions were manipulated to assess the change in values to investigate the effect of this change on participants' environmental behavioural intentions. Study 2 also examines the effect of student and national identity on the mechanisms underpinning a change in values. Chapter Five (Study 3) describes a web-based survey that assesses the effect of four individual variables (i.e., moral identity, self-efficacy, self-control, and CFC) as moderators of the link between values and environmental behavioural intentions.

Chapter Six summarises and discusses the main findings from the three empirical studies. It also discusses the theoretical implications of the empirical studies as well as the limitations of the research and suggests directions for future investigations based on the findings. 


\section{CHAPTER TWO: BRIEF LITERATURE REVIEW}

\section{Introduction}

This chapter reviews the psychological literature on human values and environmental engagement. It is not intended to be an extensive literature review but rather an introduction to the major constructs examined in this thesis. This chapter has two sections. Section 1 focuses on providing a more general review of environmental psychology, and more specifically a review of environmental engagement. In particular, Section 1 covers the definition, measurement, structure and functions of environmental engagement. Section 2 provides a brief review of different theoretical approaches on the context of human values. Moreover, it summarises the traditional theoretical frameworks in the study of values, with a specific focus on the research tradition by Schwartz.

\section{Section 1. Environmental Engagement: Conceptualisation and Measurement}

\section{Brief Overview of Environmental Psychology}

Environmental psychology is considered a recent approach in psychology that was only established as a recognised field of research four or five decades ago, although social scientists have worked on environmental issues for longer (Gifford, 2007). This area of research has gained relevance in recent years by dealing with urgent topics that are highly important for current challenges to the ecology of the planet (Gifford, 2014). It focuses on understanding the relationships between people and both natural and built environments. Although environmental problems have a long history, it is only in recent decades that more actions have been taken to address those problems and contribute to a change in the global situation. Gifford (2007) argues that it took some time for people to not only recognise environmental problems, such as sustainability, pollution, and energy shortages, but also to realise that psychology could be a great contributor to better understanding why people do or do not recycle, support environmental groups, and save energy and water.

The first person to use the term environmental psychology was Egon Brunswik, in 1943. However, Kurt Lewin is considered a leading figure in the field because it was due to the contributions of two of his students, Roger Barker and Herbert Wright, that the environment (at least the physical environment) was taken more seriously within psychology 
and the field received the title ecological psychology in 1940s. Reviews of the roots of environmental psychology note that it began in 1910 with atheoretical studies looking at the effect of noise and heat on work performance (Gifford, 2007). Early theoretical work began in 1940, picking up in 1950 and growing through the 60s and 70s. Since then, environmental psychology has been fast growing, incorporating an interdisciplinary approach and various methods of empirical investigation (see Gifford, 2007, for a review).

Broadly speaking, the main overall focus of environmental psychology is the investigation of how human emotions, cognition and behaviour affect (and are affected by) natural and human-made environments (Gifford, 2007). Due to pressing environmental problems, recent studies have focused on how particular psychological constructs, such as values (for example, Schultz et al., 2005; Williams \& Schaefer, 2013), personality (Kaiser \& Byrka, 2011; Milfont, \& Sibley, 2012), identity (Fielding et al., 2008; Matsuba et al., 2012), and time perspective (Milfont, Wilson \& Diniz, 2012), can explain environmental engagement.

\section{Defining Environmental Engagement for the Context of This Thesis}

Research has examined many constructs in relation to environmental protection, including environmental attitudes (e.g., Chun, 2009), behaviours (e.g., Schultz \& Zelezny, 1998), concern (Hansla, Gamble, Juliusson, \& Garling, 2008) and willingness to act (Nilsson, von Borgstede, \& Biel, 2004). In the present thesis, we will use the term environmental engagement to refer to the aggregated group of attitudes, behaviours, concerns and willingness to act that is positively directed towards the natural environment. Furthermore, it is necessary to provide definitions for the individual components of environmental engagement that will be used throughout this thesis, i.e., environmental attitudes, environmental behaviours, environmental concern and environmental willingness/intentions.

In this thesis environmental attitude is understood as the evaluative tendency or disposition (Eagly \& Chaiken, 1993) to respond in a favourable or unfavourable manner towards environmental problems (Milfont, 2009). Environmental attitudes consist of the collection of beliefs, effects, and behavioural intentions that a person holds about environmentally-related activities or issues (Schultz et al., 2005). In turn, environmental behaviour is defined as people's acts towards the environment, such as "I recycle", and "I turn off lights when I leave the room". 
With regard to concern, Zimmer, Stafford, and Stafford (1994) defined environmental concern as a "concept that can refer to feelings [that consumers have] about many different green issues" (p. 64). Other authors have defined environmental concern as a worry about environmental problems (e.g., Royne, Levy, \& Martinez, 2011). Examples of statements expressing concern are, "I am aware of the consequences of water pollution", and "I often read about climate change and its effect on the rise of the sea level concerns me" (Royne et al., 2011). The term environmental concern has also been associated with terms such as environmental knowledge and environmental awareness about the negative consequences of the environmental crisis and a continued interest in sustainable initiatives, such as alternative forms of energy generation (Hausbeck, Milbrath, \& Enright, 1992; Royne et al., 2011). In the literature, there is evidence demonstrating that the three terms, concern, knowledge, and awareness, can be considered independent processes that are intrinsically connected to acquire knowledge about the local or global environmental situation (Gökşen, Adaman, \& Zenginobuz, 2002). These processes make people aware of environmental issues (Royne et al., 2011), the consequences of their behaviour, as well as the possible solutions to make things better. For example, Milfont (2012) shows that knowing more about climate change increases overall concern, which in turn leads to greater sense of responsibility to help in solving climate change issues. In the present thesis, the term 'concern' refers to experiences of awareness and feelings of worry triggered by knowledge of environmental issues (Royne et al., 2011). Royne et al. (2011) proposed that these initial feelings of worry lead people to consider consequences of their actions and evaluate possible solutions to environmental issues.

With regard to willingness, it can be argued that, while environmental concern takes a cognitive and emotional approach, environmental willingness is an indicator of people's behavioural intentions (Gökşen et al., 2002). People's environmental intentions are usually assessed in relation to a positive outcome. For example, "I am willing to pay more for ecofriendly products", and "I am willing to donate money to environmental groups" (see more examples of items from the adapted GEB scale in Appendix A). Indeed, some studies point out the similarity between willingness to act and behavioural intentions (Ajzen \& Fishbein, 2005; Fishbein, 2008). Only one study attempting to distinguish between behavioural intentions, behavioural willingness and behavioural expectations was found in the literature (see Pomery, Gibbons, Reis-Bergan, \& Gerrard, 2009). However, the authors argued that intention, willingness and expectation are three very specific versions of the same construct. 
For this reason, and in order to simplify nomenclature, willingness and intention are conceptually treated as synonyms in the present thesis.

From the literature review described above, it is possible to identify the many terms used to describe feelings, thoughts, and acts towards the environment. Often these terms are used as synonyms in the literature but at other times they do refer to completely distinct concepts or measures. This difficulty in defining the key environmental concepts and the need for more consistent nomenclature across studies is a key issue for environmental psychologists (Gifford, 2007; Milfont, 2007), and of importance for the accurate measurement of these constructs. After providing separate definitions for the individual components of environmental engagement in this section, it is worth emphasising that these components are often interrelated and the relationships between them have been investigated in past research. For example, the relationship between attitudes and behaviours has been explored extensively in previous research (e.g., Fazio \& Towles-Schwen, 1999; Fazio \& Zanna, 1978; Fishbein \& Ajzen, 2005; Gollwitzer, 1999; Krosnick \& Petty, 1995; Ouellette $\&$ Wood, 1998; Strack \& Deutsch, 2004). The following section of this thesis will explain the relationships between these variables.

\section{Explaining the Relationships between Environmental Attitudes and Behaviours: Contributions from the Theory of Planned Behaviour (TPB) and Identity Research}

Different theoretical approaches have attempted to explain the relationships between pro-environmental attitudes and behaviours. For example, Kaiser, Byrka, and Hartig (2010) published a review of two different approaches that aimed to understand the attitudebehaviour link: a formal versus a causal approach. According to the formal approach, attitude and behaviour concepts can be treated as formally related - that implies treating them as aspects of a unit. In particular the formal approach argues that, "a latent attitude is a disposition to act, which becomes a manifest reality in its behavioural indicators" (p. 2). The attitude gives a subjective significance for the behaviours whereby it becomes a personal reality. On the other hand, the causal approach examines the distinction between general and specific attitudes and behaviours. According to this approach, attitudes and behaviours are distinct from each other and individual attitudes causally control behaviours. The attitude has to be triggered and then it will produce the behaviour. The causal approach can be found in many contemporary attitude models. Amongst the models that are in line with the causal approach, the TPB has been cited as a key theoretical framework explaining why individuals 
decide to perform pro-environmental acts (Inoue \& Alfaro-Barrantes, 2015). Particularly, the TPB has been one of the most remarkable models used to explain the attitude-behaviour link, and this model is thus useful to understand the relationships between environmental attitudes and behaviours discussed in this thesis.

Specifically, the TPB is a social-psychological model that proposes that intention is the most proximal determinant of an individual's behaviours. Intentions are in turn predicted by attitudes, subjective norms ${ }^{2}$ and perceived behavioural control. Perceived behavioural control is a key component of the TPB, referring to the extent to which individuals perceive their behaviour to be under their autonomous control (Fielding et al., 2014). Several studies have shown that perceived behavioural control exerts a moderation effect in the intentionbehaviour relationship (e.g., Terry \& O'Leary, 1995). Furthermore, Ajzen (1991) argues that the power of perceived behavioural control to predict intention depends on the importance of the attitude and subjective norms. It also depends on the type of behaviour and the nature of the situation. In other words, the author argues that there are no simple formulas in the prediction of social behaviour and "however strongly held, the implementation of an intention into action is at least partially determined by personal and environmental barriers" (Ajzen, 1991, p. 472). For this reason, the perceived behavioural control can be powerful when conscious control over behaviour decreases. Ajzen suggests that, in optimal conditions of complete volitional control, the relationship between intention and behaviour should also be optimal, which means that perceived behavioural control should wield a weak influence (or none) on this relationship. On the other hand, perceived behavioural control acts as a strong moderator of the intention-behaviour relationship if the behaviour is not under complete volitional control.

In the environmental psychology literature, the TPB has also been applied to better understand the influence of values and perceived control on pro-environmental acts. Studies such as the one conducted by Maio, Pakizeh, Cheung, and Rees (2009) have used the TPB as the theoretical base to explain the mechanisms that underlie value change and consequently how values affect environmental behavioural intentions. Other studies have focused on adding identity to the model to better explain environmental engagement. For example, a study conducted by Fielding et al. (2008) incorporated identity into the TPB to investigate

\footnotetext{
${ }^{2}$ Subjective norms are individuals' perceptions of whether other people would want them to perform certain behaviour (Ajzen \& Fishbein, 1980).
} 
intentions to engage in environmental activism. They measured TPB constructs, environmental group membership (i.e., group identity) and a stronger sense of themselves as an activist (i.e., self-identity) in a sample of university students. The authors found that students who were more involved with environmental groups and had a stronger sense of themselves as activists, demonstrated stronger intentions to engage in environmental activism. Other studies have also shown the important role of identity as an additional predictive variable of intention within the TPB framework (e.g., Feldman, 1984; Nigbur, Lyons, \& Uzzell, 2010; Stryker \& Burke, 2000).

To summarise, the TPB is a useful theoretical model to explain the relationships between attitudes, intentions and behaviours including those directed towards the environment. Insights provided by this model will help to explain the relationships among the environmental outcomes investigated in this thesis, especially in Study 1. The addition of perceived behavioural control and identity into the TPB to understand environmental outcomes will also contribute to important discussions in the empirical chapters of this thesis (Study 2 and Study 3).

\section{Measuring Environmental Engagement (Attitudes, Behaviours, Concern and Willingness/Intentions)}

Different types of measures have been used to assess attitudes, behaviours, concern and willingness/intentions. Traditionally, environmental attitudes have been measured using self-report methods such as interviews and more commonly, questionnaires. Less common is the use of implicit techniques, such as observations and priming techniques. The number of studies using self-reported techniques is large, for example, Milfont (2009b) reported that at least 700 measures have been used in the literature to assess environmental attitudes. Milfont (2009b) highlights three main measures of environmental outcomes that have been extensively used and had their validity and reliability checked. These are the Ecological Scale (Maloney \& Ward, 1973), the Environmental Concern Scale (Weigel \& Weigel, 1978), and the New Environmental Paradigm Scale (Dunlap, Van Liere, Mertig, \& Jones, 2000). The Ecological Scale was proposed originally by Maloney and Ward (1973) and is composed of 130 items. A shorter version consisting of only 45 items was proposed later in 1975 (Maloney, Ward, \& Braucht, 1975). The Environmental Concern Scale by Weigel and Weigel (1978) comprises 16 items, whereas the New Environmental Paradigm (NEP) scale incorporates 15 items. The NEP is considered a concise measure that shows an advantage in 
relation to the other two measures. The advantage of the NEP is the use of only general environmental items that do not become dated (Milfont, 2009).

The available measures assessing environmental behaviour have focused mainly on assessing past behaviour. Some assess broad behaviours and easy tasks while others focus on more specific behaviours and high risk tasks. The number of measures assessing environmental behaviour is large and, based on articles collected for the meta-analysis presented as Study 1 of this thesis, most researchers create their own measure of behaviour towards the environment. Because of this, it is difficult to keep track of all behavioural measures as they often are particular to a single study. However, one example of a behavioural measure that is useful for application in contexts other than the one it was developed for is the General Ecological Behaviour Scale. Developed originally by Kaiser and Wilson (2004), this measure assess a variety of different environmental acts and has good psychometric properties indicating that it is a precise and a valid measure. This measure is detailed in Chapter 4 and an adapted version will be used in the empirical studies described in this thesis.

With regard to measures assessing environmental concern and environmental willingness/intention, these are usually confounded with measures of environmental attitudes - just as the concepts are. Measures of environmental concern (e.g., Hansla et al., 2008) are more difficult to identify and distinguish from measures of environmental attitudes. However, consistent with the definition of environmental concern, a measure of environmental concern should explicitly integrate items that deal with information, awareness and feelings of worry for the environment. Measures of environmental willingness (e.g., Nilsson et al., 2004) or intention are usually similar to environmental behavioural measures but the wording of the items change (e.g., "I am willing to recycle" instead of "I recycle"), as well as the response scale (e.g., scales generally range from not willing to very willing). 


\section{Section 2. Human Values}

The investigation of values started in ancient philosophy, seeking understanding of the dichotomy between what is good and what is evil (Farley, 1990). In contemporary literature on the topic, the investigation of values is scientific and empirical, encompassing different fields such as psychology, sociology and economics. In psychology, values have been related to several outcome variables, such as: helping behaviour (Diniz, 2009), personality (Musek, 1990), sexual experience (Levine, 1997), voting intention (Caprara, Vechione, \& Schwartz, 2012), well-being (Welzel \& Inglehart, 2010), drug use (Carlson \& Edwards, 1987), and decision making (Fritzsche \& Oz, 2007), to cite a few. Values are critical in the context of this thesis as they have been shown to underpin people's environmental engagement (Maio \& Olson, 1995). The link between values and environmental engagement is a recent finding because environmental psychology is a young area of investigation. However, the study of values has a strong tradition and extensive research exists.

There are many different definitions of values. According to a review by Harding and Phillips (1986), there were around 180 different conceptualisations of values in the 1980s. Despite this diversity, all of these definitions share two commonalities that reflect the essence of the value concept, that is, 1) values are positive and 2) values express what is desirable in a social group or culture. The first statement that values are positive implies that values always have a positive connotation endorsed by a person or by a collective unit (Roe \& Ester, 1999; Rokeach, 1973), and that values have an intrinsic worth that varies for each individual. In this case, a value that is endorsed by one person may not be interpreted as something positive by another person and, as a consequence, that person would not regard that particular value as a guiding principle in his/her life. The second statement that values express what is desirable implies that values are something that is expected and considered acceptable by a group or person. The fact that values are seen as having positive connotations is implicitly linked to the concept of moral values (Vauclair, 2010), as this implies the conceptualisation of what is right or prescriptive (Kilby, 1993). Indeed, researchers agree that values contain a moral connotation (see Feather, 1996; Harding \& Phillips, 1986; Kilmann, 1981; Schwartz, 1994; Smith \& Schwartz, 1997; Rokeach, 1973).

Moreover, the study of values as positive and desirable constructs encompasses two levels of analysis that reflect two different scopes or approaches. The first approach assesses 
values at the individual level (or personal values) and the second approach assesses values at the national/cultural level (or cultural values). Different research traditions and methodologies are used when assessing personal values as opposed to cultural values (e.g., Inglehart, 1997; Hofstede, 1983). However, some of these research traditions may simultaneously investigate values at both the individual and cultural level (e.g., Schwartz, 1992; Schwartz, 2004).

Furthermore, there are several research approaches to values that are applicable to the study of environmental issues (see, Inglehart, 1997; Hofstede, 1983, 1991; Rokeach, 1973; Schwartz, 1992). In recent years, studies have mainly used Schwartz's $(1992,1999)$ model of human values to investigate the influence of values on environmental outcomes (e.g., Milfont, Sibley, \& Duckitt, 2010; Schultz, 2001; Schultz et al., 2005; Stern, Dietz, \& Kalof, 1993). The remarkable stability of the Schwartz's values model across different countries and the similarity of the patterns of values among cultures made Schwartz's values theory one of the most acknowledged theories in psychology (Schwartz, 2012). For these reasons, the current research will focus on this value theory, described in detail later in this thesis. However, before describing Schwartz's values theory, a brief review of other key research traditions on values is presented below with the aim of providing a broader overview of the main values theories. In particular, the work of Rokeach, Inglehart, and Hofstede will be reviewed as they have all had a substantial influence on our understanding of human values.

\section{Theoretical Approaches on Values: Rokeach, Inglehart, Hofstede and Schwartz}

\section{The Rokeach model}

Rokeach (1973) defined values as "enduring beliefs that a specific mode of conduct or end-state of existence is personally or socially preferable to an opposite or converse mode of conduct or end-state of existence" (p. 5). For Rokeach, values reflect concepts or modes of behaviour that serve as principles that guide people's lives and vary in importance. In Rokeach's view, values can be grouped into two categories: instrumental values (i.e., modes of conduct, such as to be helpful) and terminal values (i.e. end-states, such as equality) (Heath \& Fogel, 1978; Rokeach, 1973). Both instrumental and terminal values are each measured by 18 items on the Rokeach Value Survey (RVS). Instrumental values include for example, "ambitious (hard-working, aspiring)", and "broad-minded (open-minded)", while terminal values include "a comfortable life (a prosperous life)" and "an exciting life (a stimulating, active life)". 
Instrumental values are grouped into moral values (e.g., honesty, love) and competence values (e.g., imaginative, logical). Moral values refer to modes of behaviour and do not necessarily include values that express end-states of existence; feelings of guilt are associated with a lack of commitment to these value types (Rokeach, 1973). Competence values, also known as self-actualisation values, have a personal rather than interpersonal focus; feelings of shame are associated with failure to preserve these values (see Rokeach, 1973). On the other hand, terminal values can be distinguished from personal (e.g., world peace and brotherhood) and social values (e.g. salvation and peace of mind). Personal values encompass values that are self-absorbed, while social values include values that are societycentred.

Research using Rokeach's value system to assess the influence of values on environmental engagement is limited (Dunlap, Grieneeks, \& Rokeach, 1983; Neuman, 1986). One of the few more recent studies on the topic has shown that personal values influence environmental behaviour, such as water consumption (Pinto, Nique, Añaña, \& Herter, 2011). In this specific study, consumers with higher concern for the environment seemed to attribute more importance to personal values, particularly to conformity and personal virtues, rather than other types of values.

Rokeach (1973) was also interested in assessing value change and proposed the value self-confrontation (VSC) method, which is discussed in more detail in Chapter 4 of this thesis. This method represents one of the most important contributions of Rokeach to the study of values. Moreover, the related theoretical framework had a strong and important impact on the literature and provided the basis for the development of Schwartz's values theory (Schwartz, 1992; Schwartz \& Bilsky, 1990). The instrumental/terminal distinction of values dimensions as well as the values measure proposed by Rokeach, were used in the original article by Schwartz and Bilsky (1990). Schwartz's values theory is outlined later in this chapter.

\section{The Inglehart model}

Inglehart $(1981,1990)$ is also a pioneer in the investigation of values. He proposed that values are expressions of human needs that can be grouped into two main streams: materialistic and post-materialistic values. His theory was derived by reducing Maslow's hierarchy of needs into two basic needs which conceptually represent the extremes of a "unidimensional value continuum" (Datler, Jagodzinski, \& Schmidt, 2013). On one end of 
the continuum, materialism refers to the need for material and physical security while on the other end, post-materialism emphasises the need for freedom, self-expression, participation, or beauty (Inglehart, 1990). Later Inglehart's theory was refined and the original value continuum was renamed to survival vs. self-expression dimension, and values of interpersonal trust, happiness and liberal sexual morality were incorporated as further indicators (Inglehart, 1990). In addition to renaming the original dimension, a second dimension was added which contrasts traditional and secular societies. The two dimensions reflect a phase in history where the process of industrialisation was linked to value change in society. Datler et al. (2013) claimed that, during the change from an industrial to postindustrial society, self-expression values became the predominant values.

Inglehart's materialism/post-materialism (MPM) dimension has been criticised and reviewed over the years (Braithwaite, Makkai, \& Pittelkow, 1996), but his theory still adds significantly to the study of values, especially at a cultural level of analysis. For example, in a study conducted by Inglehart and Baker (2000), the authors used three waves of the World Values Survey (see Inglehart, 2008, for a review). The study included 65 societies representing $75 \%$ of the world's population and showed how economic development brings systematic cultural changes in values (Inglehart \& Barker, 2000).

Studies have also demonstrated the relationship between Inglehart's values and environmental engagement (Inglehart, 1995; Inglehart \& Baker, 2000). Some of the empirical studies used Inglehart's materialism and post-materialism values dimensions to explain the influence of values on local environmental concern and attitudes (Gökşen et al., 2001; Gooch, 1995). The common finding from these studies is that post-materialistic values influence environmental engagement. For example, a study carried out by Gökşen et al. (2001) showed that individuals oriented by post-materialistic values were willing to pay more for improvement in both local and global environmental problems than individuals with materialistic values. In turn, a study by Gooch (1995) showed that post-materialistic values mediate the relationship between economic factors and support for the environment. The findings revealed that, although economic factors predicted pro-environmental attitudes at a societal level more than they did at an individual level, post-materialistic values mediated the relationship between economic factors and pro-environmental attitudes at both individual and societal levels (Gooch, 1995).

Relevant to the study of environmental outcomes, Inglehart's values theory focuses more on the interpretation of what happens within societies. Although useful, researchers 
may wish to examine the influence of values and environmental engagement at the individual level. Although it is interesting to assess and reflect on the influence of political, economic and technological values, it is beyond of the scope of the present thesis to assess values on a national/cultural level.

\section{The Hofstede model}

Hofstede's $(1983,2001)$ values framework was also designed to assess culture, particularly in the context of organisations (Rinne, Steel, \& Fairweather, 2012). Hofstede conducted a comparative study among employees from more than 40 cultures. The results from his studies allowed a distinction between four cultural dimensions relating to basic societal issues (Hofstede \& Bond, 1984). The four factors described by Hofstede are listed below:

- Power distance. The extent to which a society accepts that relationships with authority are unequal so that it is legitimate that the power in organisations is distributed unequally.

- Uncertainty avoidance. Refers to ways of dealing with uncertainty. When people feel uncomfortable or threatened by an ambiguous situation, they tend to place greater value on security and conformity.

- Individualism-collectivism. The extent to which individuals care for themselves or care about the welfare of the group. Individualists value independence and selfexpression while collectivists value group interests above individual interests. Collectivists also strongly value reciprocation of favours, loyalty and maintenance of tradition.

- Masculinity-femininity. The extent to which society reinforces male or female stereotypical values. A masculine society prioritises success, money, personal accomplishments, and people are more ambitious and aggressive. A feminine society emphasises a preference for relationships, caring for others, quality of life. People in a feminine society tend to be more modest, humble and nurturing.

Later Hofstede added a new dimension to his model (Hofstede, 2001), which was first called confucian/dynamism and later labeled long-term vs. short-term orientation. The longterm values dimension refers to the orientation towards the future and rewards, including values of persistence, saving, and capacity to adapt; in contrast, the short-term values 
dimension refers to the orientation towards past/present. The long-term vs. short-term orientation includes values of stability, respect for tradition, saving face, reciprocation and satisfying social obligations.

In environmental engagement studies where Hofstede values were used to predict environmental outcomes, individualism vs. collectivism is the most commonly investigated dimension. The main prediction that has been tested is that individualistic countries tend to care less about the environment compared to collectivistic countries. As individualists concentrate on personal gains and benefits (Hofstede, 1983), they act in environmentally friendly ways to gain social approval or feel better about themselves (Cho, Thyroff, Rapert, Park, \& Lee, 2013) and not so much because they genuinely care about the environment. On the other hand, the majority of research indicates that collectivists are generally more concerned about the environment than individualists are (for example Cho et al., 2013). In collectivistic cultures, environmental concern is linked to the sense of living up to the expectation of others and being socially accepted. The study conducted by Cho et al. (2013) found strong support for this claim. However, a few contradictory results suggest that sometimes the relationship between individualistic/collectivistic values and environmental engagement may not always follow the predictable directions. For example, in another study conducted by Cho et al., results indicated there was a positive relationship between individualistic values and environmental engagement when a negative relationship was expected (Cho et al., 2013). Also, it can be argued that the individualism vs. collectivism dimension is too broad and does not look at particular aspects of collectivistic and individualistic cultures, especially in regards to the environment. For example, China is considered a collectivistic country but does not have a good environmental track record, with high levels of air pollution among other things (OECD, 2014).

In a related strand of research, other studies investigated independent and interdependent self-construals linked to environmental issues. Individuals with independent self-construal are individuals who define themselves by differentiating themselves from others, focusing on their own unique attributes (Markus \& Kitayama, 1991). These people tend to show egoistic environmental concern and competitiveness for shared resources. On the other hand, people with interdependent self-construal define themselves based on relationships (Markus \& Kitayama, 1991). They also tend to place a stronger emphasis on harmony with others and were more inclined to cooperate in the sharing of resources. In the environmental domain, Arnocky, Stroink and DeCicco (2007) proposed a third individual 
characteristic, the meta-personal self-construal, which is represented by individuals interconnected with all living things and who are self-defined through this unified connection. The authors found that meta-personal self-construal predicted biospheric environmental concern (environmental concern in situations where other species and the natural environment are under threat), ecological cooperation, and environmental conservation behaviour.

Hofstede's framework has often assessed values at a country level, with 'distance scores ${ }^{3}$ representing stable differences between two countries. Although some studies also report that Hofstede's theory has been applied using individual consumers as the unit of analysis (Blodgett, Bakir \& Rose, 2008), this theory does not clarify which personal values are related to environmental outcomes. For that reason it will not be tested in this thesis.

\section{The Schwartz model}

As mentioned above, Schwartz developed distinct theories for individual (Schwartz, 1992) and cultural levels (Schwartz, 1999) of analyses. Given the focus of the present thesis, only his individual level theory will be discussed. Schwartz (1992) defined values as transsituational benchmarks or goals organised by importance as guiding principles in one's life. He developed a broad model for classifying the dimensions of values, with value-items clustered into ten universal value types at an individual level of analysis (Schwartz, 1992, 1994). The ten value types are: power, achievement, universalism, benevolence, selfdirection, stimulation, hedonism, security, conformity, and tradition. Schwartz's (2006) description of each value type is presented below:

- Power: emphasise social status, control, prestige and dominance over resources and people;

- Achievement: emphasise personal success and competence according to social expectations;

- Universalism: emphasise appreciation, tolerance, understanding, and protection for the welfare of people and nature;

- Benevolence: emphasise preserving and enhancing the welfare of the in-group;

\footnotetext{
${ }^{3}$ The distance between two probability distributions or probability measures (Leonenko, Los \& North, 2013).
} 
- Self-direction: emphasise independent thought and action; the ability of choosing, creating, exploring;

- Stimulation: emphasise novelty, excitement and challenge in life;

- Hedonism: emphasise pleasure and sensuous gratification for oneself;

- Security: emphasise safety, harmony and stability of the self, relationships and society;

- Conformity: emphasise restraint of actions and impulses likely to upset others and violate social norms;

- Tradition: emphasise acceptance, commitment and respect of the ideas and customs of a traditional culture or religion.

These ten value types can be further classified into four higher level value categories: openness to change, conservation, self-transcendence (ST), and self-enhancement (SE) (see Figure 2.1). First, openness to change is composed of values of self-direction, stimulation, and hedonism. This dimension emphasises independent action, thought and feeling and readiness for new experiences. Second, conservation is defined by values of tradition, conformity, and security. In turn, this dimension focuses on self-restriction, order and resistance to change. Third, ST is characterised by values of universalism and benevolence. This dimension involves concern for the welfare and interest of others. Finally, SE is defined by values of power and achievement. This dimension emphasises the pursuit of self-interests. 


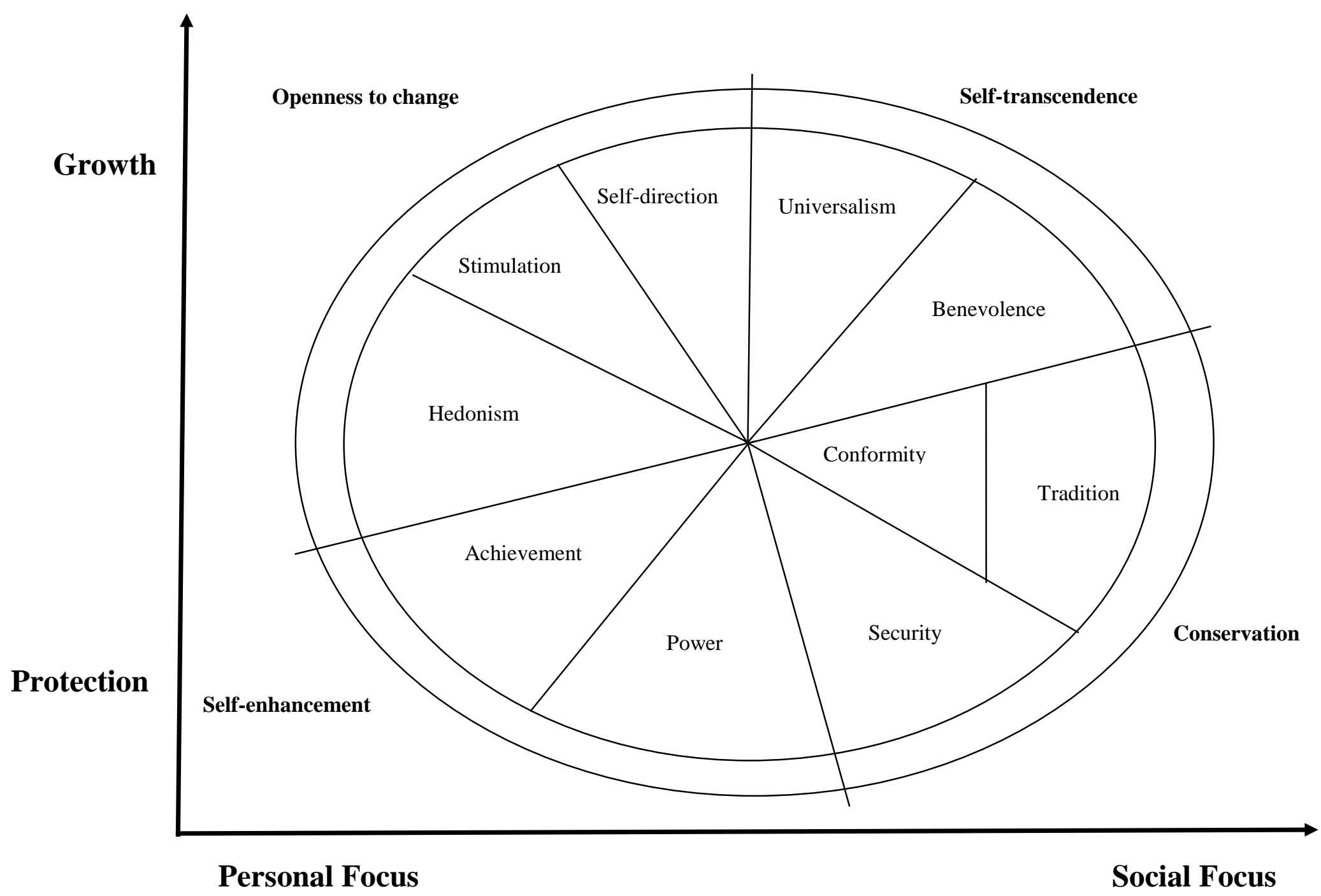

Figure 2.1. Circular model of relationships among ten motivational value types at the individual level (adapted from Schwartz, 2006, p. 965; Schwartz \& Sagiv, 1995, p. 96). 
Schwartz (1992) argues that there is a universal structure of oppositions and compatibilities between values, and his model has been replicated in more than 70 countries (Schwartz, 1992, 2006). According to his findings, individuals have the same value types but differ widely in how they prioritise different values. The two axes that congregate the ten value types into four higher order dimensions represent the progressive ascent from a personal to a social focus in value types along the $\mathrm{x}$ axis; while the $\mathrm{y}$ axis shows progressive ascent from a motivation to protect certain value types to a motivation for growth.

Figure 2.1 illustrates how the ten value types interact with each other and how they are divided into four higher order dimensions. The circle is organised by motivational similarities and dissimilarities. It represents the relationships of conflict and congruity between value types. The closer the values are to each other in any direction around the circle, the more comparable their basic motivations. Accordingly, values that are next to each other are likely to be positively correlated, whereas values at about 90 degrees are more likely to have negative or null correlations. For example, in the top right side, ST is composed of universalism and benevolence values, and is opposite to the SE values in the bottom left composed of achievement and power. According to the idea of conflicting values, a person who gives priority to ST values gives less priority to SE values because they are conflicting extremes of the same axis. The more distant the values are from each other, the more incompatible their basic motivations. Based on the conflicts and congruities observed between these ten value types, a cohesive structure of values was proposed by Schwartz (1992). This structure can be summarised with two orthogonal dimensions: 1) the ST vs. SE axis; and 2) the openness to change vs. conservation axis. For the purpose of this thesis the ST vs. SE axis is of particular interest because of its empirically tested relationship with environmental outcomes (e.g., Schultz et al., 2005), as explained in detail later in this chapter.

\section{The Schwartz Value Survey (SVS)}

To measure the ten value types, Schwartz proposed a 58 item value measure (Schwartz, 2007) called the Schwartz Value Survey (SVS). The SVS includes a comprehensive set of values that is highly suitable for cross-cultural research (Schwartz, 1992). Individuals receive a list containing each of the value items followed by its description. Respondents should rate how important each of the items is for them as a guiding principle in their lives. To test his measure, Schwartz conducted a cross-cultural study in more than 70 nations with diverse samples composed of university students, teachers and 
members of the general public. The results enabled him to establish cross-cultural stability for the items of his measure, as they clustered together in the expected dimensions in $70 \%$ of the samples (Schwartz, 1992; Schwartz \& Sagiv, 1995).

To compute indexes for each value type, it is necessary to average the ratings of the value items representative of the corresponding value type. This procedure enables value type indexes to be correlated to each other and other relevant variables. The model predicts that the correlations between values and other variables will produce a sinusoidal curve, which is a statistical representation of the circumflex structure pictured in Figure 2.1. For example, religious people tend to favour values that promote conservation of social and individual order (Tradition, Conformity, and to a lesser extent, Security) and, conversely, to dislike values that promote openness to change and autonomy (Stimulation, Self-direction); they also favour values that allow for a limited ST (Benevolence, but not Universalism), and dislike Hedonism and, to a lesser extent, dislike values that promote SE (Achievement, Power) (Saroglou, Delpierre \& Dernelle, 2004). The value-religiosity correlation fits the curve, where in the example above, the curve changes its shape following the order of the value types in the circle. Moving along the diagonal, the curve reaches a peak when the correlation scores increase and falls to a low point when the correlation decreases. Higher scores are expected between the value type and the variable of interest theoretically related to said value type (for example religiosity); low scores are expected between the opposite value type and the variable of interest. The same-shaped curve was found by Boer and Fischer (2013) with data comparing the ten value types with environmental orientations.

It is also possible to compute indexes for the four higher order values dimensions. This offers a less refined distinction between value types but is useful for a more concise interpretation of the values dimensions (for example, Maio et al., 2009). Mean scores are computed by averaging the value items that make up each of the value types. A score for conservation is calculated from the mean of items in conformity, security and traditional value types. In the same way, openness to change is computed by averaging the items that assess self-direction, stimulation and hedonistic value types. For ST, the mean of the value items that compose benevolence and universalism is used. Finally, for SE the score is computed by averaging items on power and achievement value types.

As already mentioned in previous sections of this thesis, Schwartz's measure has shown strong stability and satisfactory psychometric properties across different cultures. For 
this reason, Schwartz measure as well as his theory will be used in the present research endeavour. The next section of this chapter will discuss the critiques regarding Schwartz's theory and measure.

\section{Critiques and new developments on Schwartz's values theory and measure.}

Critiques: Although Schwartz greatly contributed to the advance of the study on values and his theory and measure are widely used, there are also criticisms. Currently, one of the main issues is the debate about whether researchers should differentiate between two levels of analyses (cultural and individual levels). The cultural level of analysis looks at the data formed by national means, while, the individual level of analysis looks at the data formed by each participant's means. If the joint value structures in the cultural and individual levels of analyses have strong enough similarities, they can be considered to have the same psychological meaning. Traditionally, research has examined the relevance of differentiating cultural and personal values. Schwartz (2010), for example, proposed that at both cultural and individual levels of analyses, the cultural and personal values should be kept "strictly theoretically and statistically independent" (Dobewall \& Rudnev, 2013, p. 48). Although Schwartz claims a differentiation between the two levels, Fischer (2012) found some similarities among structures of Schwartz's values across these levels of analysis (Fischer, 2012; Fischer \& Poortinga, 2012; Fischer, Vauclair, Fontaine, \& Schwartz, 2010).

This issue was also studied by Dobewall and Rudnev (2013) who, at both levels of analyses, were able to generally confirm Schwartz's originally proposed dimensions. However, the authors also found that the commonalities and unique components were not entirely the same across the two levels. They concluded that strict equivalence between individual and cultural levels is not fully supported. Moreover, they suggested that differentiating between the two levels of analysis is just as important as considering substantial similarities. Although, it is important to acknowledge the issue around identifying similarities and discrepancies between individual and cultural levels of analysis as one of the main criticisms of Schwartz's values theory and model, the debate around this issue is less relevant for this thesis because the focus of this work is on the individual level of analysis only.

Another important aspect of the study by Dobewall and Rudnev (2013) is the examination of the similarities between the models proposed by Inglehart and Schwartz. They found that these models theoretically overlap in both individual and cultural levels of 
analyses. In an earlier study, Wilson (2005) also showed similarities between the Inglehart and Schwartz's models at the individual level of analysis. In particular, Wilson (2005) showed that SE and ST value dimensions converge and overlap with materialism and postmaterialism. The finding that these models overlap has implications for the meta-analysis reported in the next chapter where studies using distinct measures are grouped together because the values measures are theoretically similar.

Portrait Values Questionnaire (PVQ): One of the most recent developments relating to the Schwartz's values theory and measure is the Portrait Values Questionnaire (PVQ). It is a 57 item measure with a six-point Likert-type answer scale ranging from 'not like me at all' to 'very much like me' (Schwartz, 2006; Schwartz et al., 2012). There are also shorter versions of the PVQ, for example a version composed of 21 items (Schwartz, 2003). Fundamentally, one of the main characteristics of the PVQ is that the items are written as short verbal portraits and matched to the gender of the respondent (Schwartz, 2006; Schwartz et al., 2001). Respondents indicate how similar the person described in the item is to them. The values that respondents prioritise are inferred from their self-reported similarity to people described in terms of particular values. The PVQ has also been used to assess values in studies that investigate the relationships between values and environmental engagement.

In short, Schwartz's values theory has been widely used and supported. Its values dimensions have been able to predict a large number of variables in psychological research including environmental outcomes (e.g., Boer \& Fischer, 2013). A more detailed description of the main studies relating to Schwartz's values theory and environmental outcomes is outlined below.

\section{Application of Schwartz's values theory and environmental engagement:}

\section{Self-transcendence/self-enhancement values and environmental outcomes}

Many studies have used Schwartz's values theory to assess the relationship between values and environmental engagement (e.g., Becker \& Félonneau, 2011; Feather, 2002; Fukukawa, Shafer, \& Lee, 2007; Milfont, Sibley, \& Duckitt, 2010; Nordlund \& Garvill, 2002; Schultz \& Zelezny, 1999; Schultz et al., 2005; Stern, Dietz, \& Guagnano, 1998). One of the best known studies on the topic was conducted by Schultz and Zelezny (1999) with 2,160 individuals from 14 countries (i.e., Argentina, Canada, Colombia, Costa Rica, the Dominican Republic, El Salvador, Ecuador, Mexico, Panama, Peru, Paraguay, Spain, the 
United States, and Venezuela). The authors investigated the relationship between values and environmental attitudes. Their findings reinforced the claim that ST values predict environmental attitudes, especially biospheric attitudes involving beliefs about the consequences of environmental conditions for plants and animals. Additionally, their study provided empirical support for the claim that SE values positively predict egoistic attitudes (beliefs about the consequences of environmental problems for the individual) and negatively predict biospheric attitudes (beliefs about the consequences of environmental problems for other species and the natural environment). Most importantly, this study found supportive evidence for these relationships consistently across all the countries sampled.

The link between values and environmental outcomes has been shown for environmental attitudes as well as for environmental behaviours. In a study also conducted by Schultz and Zelezny in 1998, with a smaller sample composed of five countries (Mexico, Nicaragua, Peru, Spain and The United States), the authors found evidence for the relationship between Schwartz's values and pro-environmental behaviour (such as recycling, energy conservation, water conservation, purchasing environmentally safe products, and using public transportation). In this study, the ST values were categorised into two components: nature and general. The 'nature' component consisted of three items - a world of beauty, unity with nature and environmental protection. This component measured what Stern and Dietz (1994) have labelled biospheric values. The 'general' component consisted of the remaining five ST items - broad-minded, helpful, honest, forgiving, and loyal. The results showed strong positive correlations between ST values and self-reported pro-environmental behaviour for each one of the countries investigated. Additionally, the study found a consistent pattern across countries showing strong negative correlations between SE values and pro-environmental behaviour. A few weak but significant correlations were also observed between conservation and openness to change values, and pro-environmental behaviour.

Another important article on the topic of values and environmental outcomes was also published by Schultz and colleagues in 2005 . They conducted a study among six different cultures (Brazil, Czech Republic, Germany, India, New Zealand and Russia) with around 720 participants. They assessed how Schwartz's values, specifically ST and SE values, predict concern for environmental problems and general pro-environmental behaviour. They found that, as predicted, ST values are positively related to environmental concern, while SE values are negatively related to general concern. These findings generalised across the different countries assessed in the study. 
Milfont, Sibley and Duckitt (2010) found similar results when they tested the moderating role of norm activation components on the link between values and environmental behaviour. The authors found that individuals motivated by ST values are more likely to exhibit pro-environmental behaviour if they have a high level of awareness of the impact of their actions on the environment or if they feel high levels of responsibility for the harm caused by their actions to the environment. However, for SE values, their results did not support the norm activation hypothesis, leading the authors to believe that a different mechanism is at work for this value type. Possible explanations for these results include that environmental behaviours are often portrayed by the media as difficult to perform, requiring effort and commitment with little reward. Therefore this type of behaviour is incompatible with self-interest. Another explanation is that although environmental problems are seen as serious on a global scale they may not be obvious in local communities. Furthermore, the authors argued that self-interest would only be positively associated to environmental engagement in situations that offer a clear threat to the individual.

In addition to the studies reviewed so far, other studies on environmental issues have consistently shown that ST values (e.g., social justice and a world at peace) are positively related to pro-environmental engagement, while SE values show a negative correlation to pro-environmental engagement (e.g., Bonnes, Passafaro, \& Carrus, 2011; Karp, 1996; Mirosa, Lawson \& Gnoth, 2013; Steg et al., 2014). For instance, in a meta-analysis conducted by Boer and Fischer (2013), based on both the SVS and PVQ, and using a sample of 30,357 participants from 31 countries, it was found that Schwartz's values dimensions are correlated with a diverse number of social attitudes, including pro-environmental attitudes. For the purpose of this thesis, one of the relevant findings of Boer and Fischer's (2013) study showed that pro-environmental attitudes were primarily underpinned by ST values with an average effect size of 0.35 (95\% CI [0.24, 0.46]). Additionally, Nordlund and Garvill (2002) found that not only does the ST vs. SE values dimension have a direct effect on environmental engagement, but openness to change vs. conservation values dimension is also related to environmental engagement. More specifically, the authors found that people who are motivated by high levels of openness to change are more willing to try alternative, environmentally friendly transportation (e.g., taking a train to work instead of driving a car). Although Nordlund and Garvill found evidence for a relationship between the openness to change vs. conservation dimension and environmental engagement, Schultz and Zelezny 
(1998) showed that the most pronounced results are for the relationships between ST vs. SE values dimensions and pro-environmental outcomes.

In conclusion, the studies described in this section reinforce that ST and SE values are the strongest dimension to relate to environmental outcomes. For those reasons, ST and SE values are the two main dimensions explored in the empirical studies in this thesis. The following section covers applicable methods used to measure and change values dimensions.

\section{Measuring and Changing Values}

There are different ways of assessing values in psychological research. Perhaps one of the most traditional ways of assessing values is by considering them as stable (and unchangeable) entities at a specific time point. This has been the typical approach for assessing values of individuals from different cultures in cross-sectional studies (for example, the studies conducted by Evans et al., 2013, Experiment 2; Schultz \& Zelezny, 1999; Schultz et al., 2005). Another way of assessing values is considering how they change across the years or at a certain time point. This is possible by conducting longitudinal studies such as the NZ Values Survey (Sibley, 2014) or by employing techniques that promote short term value change. Two types of techniques can be identified in the literature. The first type is the priming techniques and the second type is techniques that emphasise conflict between values. Studies using priming techniques aim to make a specific value salient and it is expected that, as a consequence, the primed value will be incorporated by the individual (e.g., Djupe \& Calfano, 2012; Mescheloff-Faran, 2010), and consequently promote short term changes in values (Djupe \& Calfano, 2012; Mescheloff-Faran, 2010).

In regards to the type of techniques emphasising conflict between values, the value self-confrontation (VSC) technique developed by Rokeach (1973) is the most commonly used. This technique works on the basis of conflict between opposed dimensions of values and it was largely employed by Maio et al. (2009), as well as explored by Schwartz and Inbar-Saban (1988). In this approach, change is actively sought by using psychological manipulations, usually experimental procedures.

Furthermore, studies have suggested that the VSC technique can promote somewhat long term changes on values, however the length of this change is still debated (see Bardi, Buchanan, Goodwin, Slabu, \& Robinson, 2014; Bardi \& Goodwin, 2011, for a review). Bardi and Goodwin argue that the emphasis in psychological literature has been on values stability, 
and that change on values can be short-term (as in response to an experiment), or long term. On the other hand, Maio et al. (2009) argue that if values are considered abstract dimensions in continuous action, it would be expected that individuals exposed to new values would be more likely to change their motivational priorities in specific conditions. By being led to rationally question their motivations (self-confrontation) and principles in life, participants would be more likely to change their values and maintain that change long term.

The VSC technique has been used with Schwartz's values items (Maio et al., 2009) producing promising results for changing values, similar to those results produced using the same technique with Rokeach's value items (Rokeach, 1973). Based on these similar outcomes and the results from studies described in the previous section of this thesis that show the relationship between the ST vs. SE values dimension and environmental engagement, it is reasonable to assume that the combination of the VSC technique using Schwartz's values items will also produce interesting results to promote environmental engagement. A detailed description of this technique is presented in Chapter 5.

The following section covers how Schwartz's values model was conceptually revised and has inspired new theoretical developments in environmental psychology that highlight environmental values dimensions.

\section{Development of a Model of Environmental Values}

Even though Schwartz successfully proposed a model that can be applied to the study of environmental outcomes, some environmental psychologists suggested that there was a need to give better coverage and greater emphasis to environmental values (Stern et al., 1993). Therefore, Stern and Dietz (1994) proposed the 'values basis of environmental concern' model. The outline of this model started with a study by Stern et al. (1993). According to the authors, the basis of their 'Schwartz-derived model' is that proenvironmental attitudes fall within a general altruistic value orientation towards the welfare of others (similar results were also found by Dobewall \& Rudnev, 2013). They stated that "environmentally relevant behaviour can reflect a trade-off between altruistic and egoistic motivations and, therefore, egoistic value orientations as well as social-altruistic ones are implicated in environmental attitudes and behaviours" (Stern et al., 1993, p. 325). Although the egoistic and social-altruistic dimensions proposed by Stern et al. (1993) are aligned with the ST vs. SE values dimension proposed by Schwartz, Stern et al. identified the need to 
incorporate human beings, as well as "non-human species or the biosphere itself" (p. 325) into the model.

In their presentation of the theoretical model, Stern and Dietz (1994) proposed that value orientation leads to information seeking and, consequently, the development of beliefs about the consequences of actions. According to this assumption, people accept information selectively because "values act as filters for information" (p. 68). For example, someone who places value on economic development above any other social goal is more likely to accept information that suggests that environmental protection can be reconciled with economic goals. On the other hand, someone who prioritises the beauty of natural landscapes would be more prone to accepting information supporting beliefs that any environmental change offers a threat to that value (Stern \& Dietz, 1994).

Based on these premises, Stern et al. (1993) and Stern and Dietz (1994) proposed three value orientations towards the environment: egoistic, humanistic (or social-altruistic) and biospheric. These orientations are compatible and may be related to the extent that people's attitudes towards the environment reflect a combination of the three. Also, according to previous research, although these orientations are more frequently noted in Western literature on environmental concern, they may also be salient in other cultural contexts (see work by Schultz et al., 2005; Stern et al., 1993).

The egoistic value orientation is likely to produce environmental concern in a situation where protection of the environment would have benefits for the individual. In this case, the benefits may overshadow any potential costs the environmentally friendly act may incur to the individual. In contrast, a socio-altruistic values orientation is likely to produce environmental concern in conditions where the protection of other human beings is involved; because of this, the individual would accept personal costs to protect the environment. An example of socio-altruistic values orientation is when people became concerned about the effects of global warming when they see other people affected by natural disasters due to increases in global temperature (Stern \& Dietz, 1994). The environmental behaviour concerned with this orientation such as recycling, would be common among people who also engage in other forms of altruism, such as blood donation (Diniz, 2009). In turn, the biospheric value orientation can trigger environmental concern in situations where other species and the natural environment are under threat. An example of biospheric value 
orientations is the work of "deep ecologists", people with strong moral principals regarding safeguarding plants and animals (Stern \& Dietz, 1994; Stern et al., 1993).

According to Stern et al. (1993), a motivation to act is represented by an equation relating the egoistic, socio-altruistic and biospheric value orientations: $\mathrm{M}=\mathrm{V}_{\text {ego }} \mathrm{AC}_{\text {ego }}+$ $\mathrm{V}_{\text {soc }} A C_{\text {soc }}+\mathrm{V}_{\text {bio }} A C_{\text {bio, }}$ where $A C$ represents the beliefs about consequences for a valued object and $\mathrm{V}$ is the weight of the value orientation towards that object. AC and V are summed across value orientations and the final product is the motivation to act. The model and equation are inspired by the Schwartz norm-activation theory. While in the original model proposed by Schwartz the variables "ascription of responsibility and "personal norms" mediated the relationship between $\mathrm{AC}$ and behaviour, these two variables were not included in Stern's model.

The method to assess value orientations based on the Stern and Dietz's (1994) model is to ask individuals to express an attitude or action regarding an environmental phenomenon. The individuals automatically review their beliefs about the phenomenon and consider whether it affects the things they value, in a process where value-expectancy relationships are in place. More recently, Stern et al. (1998) proposed a brief inventory of values composed of four 3-item scales derived from Schwartz's 56 item instrument. This inventory measured the four main clusters of ST, SE, openness to change and conservation values, and produced acceptable reliability in the prediction of pro-environmental attitudes and actions. Another important finding was the ability to discriminate between biospheric and altruistic value orientations in a sample of environmental activists, although this distinction was not possible in earlier studies (Stern et al., 1993; Stern \& Dietz, 1994) with samples from the general population. In the literature on Stern's model, Schwartz's ST values are expressed by the relationship between social-altruistic and biospheric orientations, while his SE values are expressed by the egoistic orientations proposed by Stern and Dietz (1994).

Further developments of the Stern and Dietz's (1994) value-basis theory of environmental issues can be found in the literature. For example, Schultz (2001) proposed a tripartite model of environmental concern composed of three factors which are intrinsically related to egoistic, altruistic and biospheric value orientations. Schultz confirmed these three factors of environmental concern using a sample of college students from ten different countries, providing cultural stability for his model. It is also important to mention that De Groot and Steg $(2007,2008)$, developed a similar value orientation measure based on 
Schwartz's values scale (1992, 1994), assessing egoistic, altruistic and biospheric value orientations through 13 value items. The structure and measure proposed by these authors is similar to the previously described measure proposed by Stern (Stern \& Dietz, 1994). Furthermore, in studies conducted by De Groot and Steg (2008, 2010), the results showed a pattern where people who were altruistically and biospherically oriented were more likely to act pro-environmentally, whereas individuals who endorsed egoistic values were less likely to act in a pro-environmental way. These results mirrored previous results by Stern and Dietz (1994). In addition, De Groot and Steg (2008) found that biospheric values were more strongly related to pro-environmental intentions and behaviours than altruistic values. These findings also confirmed previous findings by Stern et al. (1998).

In summary, the Stern and Dietz's (1994) values model of environmental concern expressed the assessment of environmental value orientations. This model drew from Schwartz's values theory by splitting the ST dimension into two related orientations, socialaltruistic and biospheric orientations. Furthermore, Stern's model also assessed Schwartz's SE dimension labelling it as egoistic orientation. Openness to change and conservation values are also proposed by Stern's model following Schwartz's values theory. The Stern and Dietz's (1994) model of environmental values is widely used in environmental psychological research with researchers developing upon its ideas (De Groot \& Steg, 2007, 2008; Schultz, 2001). It is the third most important model in the study of values and environmental engagement, following Schwartz and Inglehart's values models (see the meta-analysis review in the next chapter) and is one of the prevailing applications of the Schwartz's values theory and items regarding the environment.

\section{Conclusions}

This chapter reviewed the literature on environmental engagement and values. More specifically, the review focused on defining environmental attitudes, behaviours, concern and willingness/intentions, all components of one broad term called environmental engagement. The conceptualization of these environmental outcomes is still an issue in the literature. While the literature can make it difficult to distinguish these constructs, the succinct definitions presented in this chapter enable them to be accurately and effectively investigated. This chapter also explained that the Theory of Planned Behaviour (TPB, Ajzen, 1991) helps to understand the relationships between some environmental outcomes such as environmental attitudes and behaviours. Additionally, the inclusion of perceived behavioural control and 
identity into the TPB has contributed to explain the intention-behaviour relationship. Furthermore, the literature suggests that people may be more willing to perform proenvironmental acts if they feel their behaviour is under their autonomous control and if they identify themselves in an environmentally friendly way. Moreover, the reliable measurement of environmental outcomes is of fundamental importance, and a number of measures are available in the literature for that purpose.

This review has also addressed values, one of the main predictors of environmental outcomes. Specifically, this chapter described Rokeach, Inglehart, Hofstede and Schwartz's theories to explain values. These perspectives are discussed regarding conceptualization, measurement, structure and functions of values. Particularly, the present research emphasised Schwartz's values theory, as the ST and the SE dimensions of this theory has been largely used to study environmental engagement. Furthermore, care was taken to discuss the critiques and new developments in Schwartz's values theory and measure, such as the PVQ. Most importantly, Schwartz's values theory can be applied to explain environmental outcomes. From this review it can be concluded that the majority of studies described in this chapter have shown that values predict environmental outcomes. Additionally, Schwartz's values theory, specifically ST and SE values, are the most commonly used dimensions in the study of the relationships between values and environmental issues. Importantly, value change can be possible with the use of techniques such as the VSC technique. Furthermore, Schwartz's values theory has been developed further by Stern and colleagues to specifically highlight the environmental aspect of values orientations. The present thesis addresses the relationship between Schwartz's values theory and environmental outcomes using a series of empirical studies as reported in the next three chapters. Broadly speaking, this thesis predicts that ST values will be reliably and positively related to environmental outcomes, while SE values will be reliably and negatively related to environmental outcomes. More specific predictions are presented and tested in each of the empirical studies that follow in the next chapters. 


\section{CHAPTER THREE: STUDY $1^{4}$}

\section{A Meta-analysis on the Relationships between Values and Environmental Engagement}

As noted previously in this thesis, values are an important variable to be considered when explaining people's attitudes and behaviours towards the environment. To date, many studies have examined how particular dimensions of values relate to people's environmental engagement. Quite often the relationships between these constructs differ in terms of strength and also in terms of the environmental variable considered, such as attitudes, behaviours and concern (e.g., Grunert \& Juhl, 1995; Kelly, Tovey \& Faughnan, 2007; Milfont, Duckitt, \& Wagner, 2010; Schultz \& Zelezny, 1999, 2003). For example, research conducted by Schultz et al. (2005) has shown that there is a strong relationship between values and attitudes, while the relationship is weaker between values and behaviours (average effect size for ST values: .27 and .19; and SE values: -.16 and -.08 respectively for environmental attitudes and behaviours). The finding that values have a stronger association with attitudes than with behaviours is consistent with previous research that claims that values are better predictors of people's attitudes than they are of people's behavioural intentions (see Ajzen \& Fishbein, 1980; Homer \& Kahle, 1988; Milfont, Duckitt, \& Wagner, 2010). However, a study conducted by Lévy-Leboyer, Bonnes, Chase, Ferreira-Marques, and Pawlik (1996) showed a more positive and stronger relationship between values and behaviours than between values and attitudes. Overall, these findings suggest that it is important to examine the relationship between values and distinct measures of environmental engagement, such as attitudes and behaviours, as the influence of values might differ across environmental outcomes.

Despite an increasing number of studies examining the direct relationships between values and environmental outcomes, only a couple have systematically reviewed the extent to which values do indeed influence or relate to individuals' environmental engagement. As discussed in Chapter 2, two recent meta-analyses examined the associations between values and environmental outcomes. Hurst, Dittmar, Bond and Kasser (2013) focused on a particular

\footnotetext{
4 Parts of this study were presented in two international conferences. See Diniz, Fischer, Milfont, and McClure (2012) and Diniz, Milfont, Fischer and McClure (2013a).
} 
set of values (materialistic values), and found a significant medium-sized negative association between materialistic values and both environmental attitudes and behaviours (-.22 and -.24 , respectively). In addition, Boer and Fischer (2013) provided a meta-analytical review of the associations between the Schwartz's values and general social attitudes, including environmental attitudes. They showed that ST vs. SE values relate positively to proenvironmental and pro-social attitudes (average amplitude of .35). Additionally, Boer and Fischer (2013) found that ecological and cultural factors influence the value-attitude link. However, their results also showed that economic development or the country of the participants was not associated with variations in the value-attitude link.

These recent meta-analyses support the link between values and environmental outcomes, showing that the relationship between these variables yield small to medium effect sizes (Boer \& Fischer, 2013; Hurst et al., 2013). However, these studies focused only on two specific sets of values (i.e., materialistic values and Schwartz's values dimensions). The present meta-analysis extends the previous meta-analyses by focusing not only on measures that assess values using the Schwartz's values model or a specific set of values such as materialistic values. This study will offer a broader review that captures other value measures that are found in this specialised literature, and have been used to explain environmental engagement. In addition, the present study assesses a broader range of environmental outcomes. Whereas the meta-analysis conducted by Boer and Fischer (2013) only assessed environmental attitudes and the study conducted by Hurst et al. (2013) assessed attitudes and behaviours towards the environment, the present meta-analysis examines not only attitudes and behaviours, but also concern, and willingness/intentions to protect the environment.

To address these gaps in previous studies and provide a more comprehensive summary of the field, this meta-analysis will be performed to systematically assess the relationship between any theory and measure of values (going beyond assessing the narrow use of only materialistic values and Schwartz's values dimensions) and environmental engagement (attitudes, behaviours, concern and willingness). By definition, a meta-analysis is "the statistical analysis of a large collection of analysis results for the purpose of integrating the findings" (Glass, 1976, p. 3) and, therefore, it can help researchers and the general public to get an overall and more precise picture of the associations between particular constructs. It is hoped that this procedure will assist us to get a broad idea of the associations between values and environmental engagement. 
For the present study, it is expected that a positive and strong relationship will be found between ST values and environmental outcomes (attitudes, behaviours, concern and willingness). Moreover, a stronger positive correlation is expected between ST values and more general measures of environmental outcomes (environmental attitudes, concern and/or willingness) than between ST values and environmental behaviours, which would be in line with previous research (e.g., Milfont, Duckitt, \& Wagner, 2010). It is also hypothesised that a negative correlation will be found between SE values and environmental outcomes (attitudes, behaviours, concern, and willingness). Again, it is expected that this negative relationship will be stronger between SE values and environmental attitudes, concern and/or willingness than between SE values and environmental behaviours.

\section{Method}

\section{Literature Search}

A literature search of all studies, involving values and environmental engagement published until April 2012 was conducted using three different methods. First, studies were located through an electronic database search of PsychInfo and ProQuest using relevant search terms or keywords following APA guidelines (American Psychological Association, 2012) of scientific terms (i.e., values, world view, environmental attitude, environmental behaviour, ecological behaviour, and conservation). The search on electronic databases resulted in 470 references. Second, data requests were sent to electronic mailing lists for organizations related to environmental psychology, such as the International Association of Applied Psychology (Division of Environmental Psychology), and the International Society for People-Environment Studies; as well as other associations related to social and crosscultural psychology, such as the European Association of Social Psychology, and the International Association of Cross-Cultural Psychology. Finally, emails were sent directly to authors of relevant articles asking for any other unpublished data they might have. Replies from electronic mailing lists and direct email contacts resulted in 21 references, including unpublished datasets.

\section{Inclusion-exclusion Criteria and Coding of Study Characteristics}

Two main inclusion criteria were used in order to select studies for the meta-analysis. First, all studies were required to include measures of both human values and environmental outcomes. The values measures identified in the different studies assessed various values 
dimensions, but most studies identified in the literature used the Schwartz's model or related models. To simplify the presentation, and given that most of the dimensions presented in the studies could be labelled as ST and SE values dimensions, the Schwartz's model was used to classify studies using other value models. To illustrate, the Inglehart's post-materialistic vs. materialistic values were labelled as ST vs. SE values, respectively. Examples of values instruments included in this meta-analysis and the number of studies (represented by k) using the particular values instruments are listed below:

1) The Schwartz Value Survey ( $k=49$; SVS; Schwartz, 1992), which is composed of around 50 items measuring four dimensions: ST, SE, conservation and openness to change. For the purpose of this study, only the ST and SE values dimensions were considered;

2) The Portrait Value Questionnaire ( $k=1$; PVQ; Schwartz et al., 2001), which is a distinct values measure composed of 40 items measuring the same ten motivational types and four dimensions proposed by Schwartz's model. In this measure, each item describes a person briefly, forming portraits that correspond to a specific value. Again, in the present study only the ST and SE values dimensions were considered;

3) The Brief Inventory of Values ( $k=3$; Stern et al., 1998), which is composed of 15 items based on Schwartz's values model. These items represent six dimensions: ST, SE, conservation, openness to change, altruistic and biospheric. Only the ST, $\mathrm{SE}$, altruistic and biospheric values dimensions were considered in the present study;

4) Stern's Environmental Values measure $(k=6)$ proposed by Stern et al. (1995), which covers only one dimension called biospheric-altruistic value orientation. This dimension is similar to Schwartz's ST values dimension being the only one considered in the present study;

5) Rokeach Values Survey $(k=1)$, which is composed of 36 items and was originally proposed by Rokeach (1973). This measure covers six dimensions: ideal world, conformity, emotional stability, conservation, personal virtues, and exciting life. These values were combined in the present study and theoretically represented three dimensions of values proposed by Schwartz (i.e., ST, conformity and openness to change). In this way, ideal world was categorised in the ST values 
dimension; conformity, conservation and emotional stability were categorised in the conformity values dimension; and personal virtues, and exciting life were categorised in the openness to change values dimension. In the present study only the ideal world dimension representing the ST values dimension was considered;

6) The Quality of Life Indicators ( $k=1$ ), developed by Poortinga et al. (2004) and composed of 22 indicators of participants' life. The indicators were developed based on Rokeach and Schwartz's values and also comprised environmental values. This measure encompasses quality of life aspects of aesthetic beauty, challenge/excitement, change/variation, comfort, education, environmental quality, freedom, health, identity/self-respect, leisure time, material beauty, money/income, nature/biodiversity, partner and family, privacy, safety, security, social justice, social relations, spirituality/religion, status/recognition, and work. Only the quality of life aspects representing SE values dimension (e.g., status/recognition) and ST values dimension (e.g., social justice) were considered for the present study.

7) The Value Orientations $(k=2)$, an adapted version of the Schwartz Value Survey developed by De Groot and Steg $(2007,2008)$ that comprises 13 values measuring egoistic, altruistic, and biospheric value orientations. Egoistic values were categorised as representing the SE values dimension, while both altruistic and biospheric values were categorised in the ST values dimension and these were the values considered in the present study;

8) The Allport-Lindzey Study of Values $(k=1)$ (Allport, 1960), which includes theoretical, economic, aesthetic, social, political, and religious dimensions. For the present study, only the social dimension was treated as representing the ST values dimension and considered for the present meta-analysis;

9) Inglehart's Post-materialistic Values measure $(k=26)$ was proposed by Inglehart (1990). This measure assesses respondents' preferences for the following societal goals: maintaining law and order in the country, fighting rising prices, giving people more say in important political decisions, and protecting freedom of speech. The first two goals measure materialistic values, and the second two goals measure post-materialistic values. Materialistic values were treated as representing the SE values dimension while post-materialistic values were judged to relate to 
ST values dimension proposed by Schwartz (1994) and these were the values considered in the present study.

The studies reported in this meta-analysis presented a variety of environmentallyrelated variables. To make it more parsimonious for the meta-analysis, these variables were coded into attitudes $(k=29)$, behaviour $(k=62)$, concern $(k=30)$, and/or willingness $(k=$ 16). To enable the accurate selection of and distinction between these four dependent variables, a list was created of all the different types of environmental outcomes assessed in the articles considered for this meta-analysis. Then, and in order to validate these four categories, a list of all environmental variables coded in the meta-analysis was presented to two experts in the field and they were asked to group the measures into the four categories (attitudes, behaviours, concern and willingness). The experts were also asked to recommend any other categories. By doing this, all the environmental outcomes were grouped into similar clusters - therefore making the meta-analysis possible. This procedure is recommended by Lipsey and Wilson (2001) and Rosenthal and DiMatteo (2001).

The measures of attitudes included, for example, the New Environmental Paradigm (NEP Scale; Dunlap \& Van Liere, 1978) comprising 12 items. The NEP Scale assesses environmental orientation, although sometimes it was described as measuring concern, attitudes and values. A revised version of this measure contains 15 items and was proposed by Dunlap et al. (2000). The 15 item version is considered the "most widely used environmental measure of values or attitudes worldwide" (Anderson, 2012, cited in Hawcroft \& Milfont, 2010, p. 261). Measures of behaviour included the frequency of conservation activities, such as turning off the lights when leaving the room. Measures of concern included, for instance, the degree to which participants are concerned about harmful effects of environmental problems for themselves, other people, and plants and animals (Schultz, 2001). Measures of willingness included, for example, the extent to which individuals are willing to donate money to an environmental organisation, or to vote for parties that promote environmental protection. Any studies in which measures of human values or environmental engagement could not be distinguished from other unrelated variables were excluded (e.g., instruments combining all different dimensions of values in one overall score; studies using the NEP scale as a measure of values and not as a measure of attitudes).

To meet the second inclusion criteria, studies had to report correlation coefficients $(r)$ between the variables or report relevant statistics to be used as the effect size measure. 
Included studies therefore comprised those reporting correlation coefficients for the relationship between values and environmental attitudes and/or behaviours, or studies reporting other relationship indices with the potential to calculate a correlation coefficient (e.g., $F$-values, $t$-values, Betas, etc.). Correlation coefficients $(r)$ were used due to their prevalence in meta-analyses and because they are the common statistic reported by the majority of studies selected for the current meta-analysis. Furthermore, Rosenthal and DiMatteo (2001) also recommend using correlation coefficients as indicators of effect size due to their properties that allow easier calculations.

Finally, any negative correlation coefficients that indicated a negative score on environmental engagement (i.e., a higher score on the measure meant low environmental engagement) were reversed. Using this method, all environmental engagement measures were guaranteed to assess environmental acts in the same direction. Non-significant correlations were also coded.

\section{Final Meta-analysis Data Set}

From the 470 studies initially identified through online databases, 434 were excluded because they did not fit the criteria described above (i.e., studies had to contain measures of both human values and environmental outcomes and also, studies had to report correlation coefficients between the variables or relevant statistics to be used as the effect size measure). The most common reasons for exclusion were that most of the studies were theoretical or qualitative and did not present statistical indicators that could be used for the transformations conducted in the meta-analysis.

The final data set contained a total of 36 independent studies (34 published articles and 2 unpublished raw data-sets). Some studies included in the meta-analysis reported information from multiple samples of participants. Each sample was then considered independently. Close to one hundred samples were included in the meta-analysis $(k=90)$, representing a total of 47,660 participants from 41 countries. A list of the studies included in the meta-analysis and coding variables is presented in Table 3.1. A summary of the samples' characteristics is presented in Table 3.2. 
Table 3.1

Studies Included in the Meta-analysis and Coding Variables

\begin{tabular}{|c|c|c|c|c|c|}
\hline Study & Country & Sample type & $\begin{array}{l}\text { Sample } \\
\text { size }(n)\end{array}$ & Values measure & Environmental outcomes measure \\
\hline Axelrod (1994) & $\overline{\text { USA }}$ & University students & 117 & Environmental orientations (Axelrod, 1994) & Environmental attitudes \\
\hline $\begin{array}{l}\text { Becker \& Félonneau, } \\
\text { (2011) }\end{array}$ & France & University students & 191 & $\begin{array}{l}\text { Portrait Value Questionnaire (PVQ; Schwartz et al., } \\
\text { 2001) }\end{array}$ & $\begin{array}{l}\text { Ecologic behaviour and environmental } \\
\text { attitudes }\end{array}$ \\
\hline $\begin{array}{l}\text { Bonnes, Passafaro, \& } \\
\text { Carrus (2011) }\end{array}$ & Italy & General population & 500 & Schwartz Value Survey (Schwartz, 1992) & General proenvironmental attitudes \\
\hline $\begin{array}{l}\text { Branzei, Vertinsky, } \\
\text { Takahashi, \& Zhang (2001) }\end{array}$ & China & $\begin{array}{l}\text { Senior executives and environment } \\
\text { managers }\end{array}$ & 300 & Environmental values (Branzei et al., 2001) & $\begin{array}{l}\text { Environmental attitudes and environmental } \\
\text { training }\end{array}$ \\
\hline Chun (2009) & China & $\begin{array}{l}\text { Employees from energy companies of } \\
\text { coal mining and washing, electricity, } \\
\text { and aluminum }\end{array}$ & 472 & Schwartz Value Survey (Schwartz, 1992) & Environmental attitudes scale \\
\hline Coelho et al. (2006) & Brazil & University students & 208 & Schwartz Value Survey (Schwartz, 1992) & $\begin{array}{l}\text { Ecocentric and antropocentric attitudes scale } \\
\text { (Thompson \& Barton, 1994; Schultz \& } \\
\text { Zelezny, 1999) }\end{array}$ \\
\hline Collins \& Chambers (2005) & Australia & University students & 205 & Brief inventory of values (Stern et al., 1998) & Preference for public transport \\
\hline Collins et al. (2007) & Netherlands & Costumers at supermarket & 198 & Schwartz Value Survey (Schwartz, 1992) & $\begin{array}{l}\text { Beliefs on environmental sustainability and } \\
\text { environmental behaviour }\end{array}$ \\
\hline De Groot \& Steg (2010) & Netherlands & University students & $\begin{array}{l}304 \\
520\end{array}$ & Value orientations (De Groot \& Steg, 2007, 2008) & Pro-environmental intentions \\
\hline $\begin{array}{l}\text { Deng, Walker \& } \\
\text { Swinnerton, G. (2006) }\end{array}$ & Canada & General population & 160 & Environmental values (Stern et al., 1995) & NEP \\
\hline $\begin{array}{l}\text { Deng, Walker \& } \\
\text { Swinnerton, G. (2006) }\end{array}$ & China & General population & 178 & Environmental values (Stern et al., 1995) & NEP \\
\hline $\begin{array}{l}\text { Fatos, Fikret, \& Unal } \\
(2001)\end{array}$ & Turkey & General population & 1565 & The postmaterialism scale (Inglehart, 1990) & Environmental concern measure \\
\hline Feather (2002) & Australia & General population & 324 & Schwartz Value Survey (Schwartz, 1992) & Environmental attitudes \\
\hline Fukukawa et al. (2007) & USA & University students & 100 & Schwartz Value Survey (Schwartz, 1992, 1994) & $\begin{array}{l}\text { Social and environmental accountability scale } \\
\text { (SEA) - (CDCAC, 2002; Fukukawa et al., } \\
\text { 2007) }\end{array}$ \\
\hline Gooch (1995) & $\begin{array}{l}\text { Sweden } \\
\text { Latvia } \\
\text { Estonia }\end{array}$ & General population & $\begin{array}{l}278 \\
407 \\
400\end{array}$ & The postmaterialism scale (Inglehart, 1990) & Environmental concern \\
\hline Hansla et al. (2008) & Sweden & General population & 494 & Schwartz Value Survey (Schwartz, 1992) & $\begin{array}{l}\text { Environmental concern measure (Schutz, 2001) } \\
\text { and awareness of consequences measure } \\
\text { (Garling et al., 2003) }\end{array}$ \\
\hline $\begin{array}{l}\text { Kaiser, Ranney, Hartig, \& } \\
\text { Bowler (1999) }\end{array}$ & Swiss & General population & 445 & Environmental values (Kaiser et al., 1999) & $\begin{array}{l}\text { General Ecological Behaviour scale (GEB), } \\
\text { environmental knowledge and ecological } \\
\text { behavioural intention }\end{array}$ \\
\hline $\begin{array}{l}\text { Kaiser, Ranney, Hartig, \& } \\
\text { Bowler (1999) }\end{array}$ & USA & University students & 488 & Environmental values (Kaiser et al., 1999) & $\begin{array}{l}\text { GEB scale, environmental knowledge and } \\
\text { ecological behavioural intention }\end{array}$ \\
\hline $\begin{array}{l}\text { Kemmelmeie,Król \& Kim } \\
(2002)\end{array}$ & Australia & General population & 1465 & The postmaterialism scale (Inglehart, 1990) & Environmental willingness \\
\hline
\end{tabular}




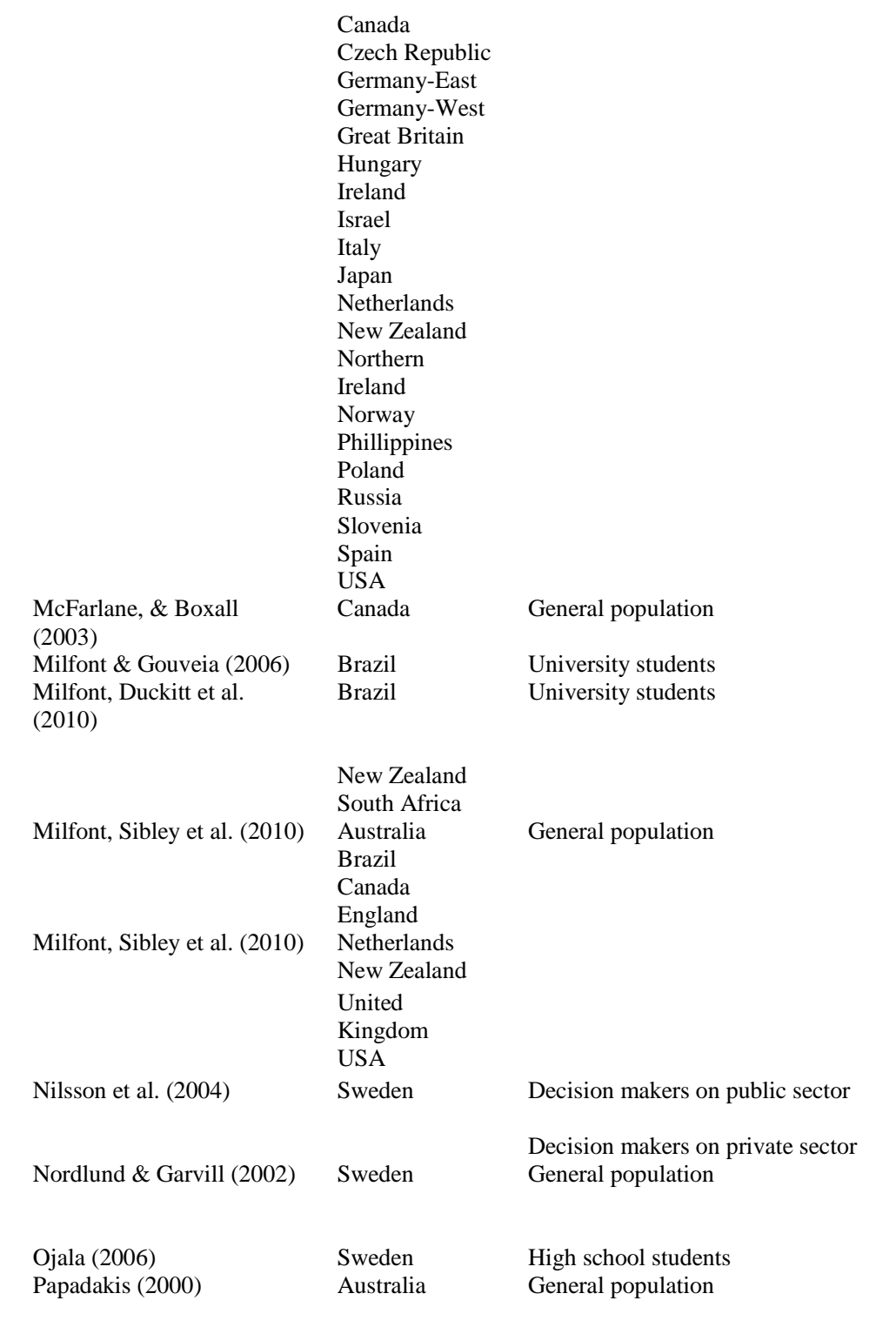

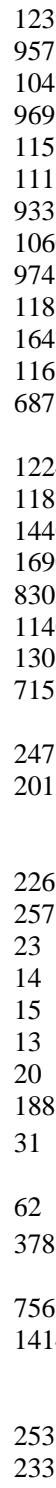

Value orientation (McFarlane \& Boxall, 1996, 1999)

Brief inventory of values (Stern et al., 1998)

Schwartz Value Survey (Schwartz, 1992; 1994)

Schwartz Value Survey (Schwartz, 1992)

Schwartz Value Survey (Schwartz, 1992)

Schwartz Value Survey (Schwartz, 1992) and Thompson \& Baron (2004) anthropocentric an ecocentric values

Schwartz Value Survey (Schwartz, 1992)

Ecocentric and utilitarian values
Environmental attitudes and environmental behaviours

Preservation Scale (Milfont 7 Duckitt, 2004)

Environmental Attitudes Inventory, Ecological

Behaviour Scale and Perceived Environmental
Threat Scale

Self-reported Environmental Behaviour Scale

Willingness to accept policies to reduce negative climate change effects

Pro-environmental behaviours

Worry about environmental risks

Environmental political participation (voting intention for green party) 


\begin{tabular}{|c|c|c|c|c|c|}
\hline Pinto et al. (2011) & Brazil & General population & 400 & Rokeach Value Survey (RVS - Rokeach, 1973) & $\begin{array}{l}\text { Environmental awareness (attitudes) and } \\
\text { Wasteful habits (behaviour) }\end{array}$ \\
\hline $\begin{array}{l}\text { Poortinga, Steg \& Vlek, } \\
\text { (2004) }\end{array}$ & Netherlands & General population & 455 & $\begin{array}{l}\text { Quality of life indicators (list based on Rokeach } \\
\text { (1973), Schwartz (1992) values and environmental } \\
\text { values (Poorting, Steg \& Vleg, 2004) }\end{array}$ & $\begin{array}{l}\text { NEP, Concern about Global Warming (CGW) } \\
\text { Scale, environmental behaviour and } \\
\text { environmental attitudes }\end{array}$ \\
\hline $\begin{array}{l}\text { Rabinovich et al. (Study } 2 \text {, } \\
\text { 2009) }\end{array}$ & Not stated & General population & 152 & $\begin{array}{l}\text { Value of collective environmental actions } \\
\text { (Environmental values, Milfont \& Duckitt, 2004) }\end{array}$ & $\begin{array}{l}\text { Environmental behaviour and environmental } \\
\text { attitudes }\end{array}$ \\
\hline $\begin{array}{l}\text { Raymond, Brown, \& } \\
\text { Robinson, (2011) }\end{array}$ & Australia & General population & 1323 & Environmentally relevant items & Intention on planting of native vegetation \\
\hline Rioux (2011) & France & Pupils & 162 & Brief inventory of values (Stern et al., 1998) & Battery collection behaviour \\
\hline \multirow[t]{2}{*}{ Schultz \& Zelezny (1998) } & Mexico & University students & 187 & Schwartz Value Survey (Schwartz, 1992; 1994) & Environmental behaviour \\
\hline & $\begin{array}{l}\text { Nicaragua } \\
\text { Peru }\end{array}$ & & $\begin{array}{l}78 \\
160\end{array}$ & & \\
\hline \multirow[t]{2}{*}{ Schultz \& Zelezny (1998) } & Spain & University students & 187 & Schwartz Value Survey (Schwartz, 1992; 1994) & Environmental behaviour \\
\hline & USA & & 345 & & \\
\hline \multirow[t]{7}{*}{ Schultz (Study 4; 2001) } & Colombia & University students & 149 & Schwartz Value Survey (Schwartz, 1992; 1994) & Self-report proenvironmental behaviour scale \\
\hline & Costa Rica & & 213 & & \\
\hline & El Salvador & & 194 & & \\
\hline & The Dominican & & 121 & & \\
\hline & Republic & & & & \\
\hline & Ecuador & & 201 & & \\
\hline & Panama & & 100 & & \\
\hline \multirow{4}{*}{ Schultz (Study 4; 2001) } & Paraguay & University students & 200 & Schwartz Value Survey (Schwartz, 1992; 1994) & Self-report proenvironmental behaviour scale \\
\hline & Peru & & 224 & & \\
\hline & Spain & & 104 & & \\
\hline & Venezuela & & 194 & & \\
\hline \multirow[t]{2}{*}{ Schultz et al. (2005) } & Brazil & University students & 208 & Schwartz Value Survey (Schwartz, 1992; 1994) & $\begin{array}{l}\text { Environmental behaviour and environmental } \\
\text { concern }\end{array}$ \\
\hline & Czech Republic & & 113 & & \\
\hline \multirow[t]{4}{*}{ Schultz et al. (2005) } & Germany & & 120 & & \\
\hline & India & & 210 & & \\
\hline & New Zealand & & 217 & & \\
\hline & Russia & & 120 & & \\
\hline Shean, \& Shei (1995) & USA & Volunteers from environmental groups & 62 & The Allport-Linzey study of values (1960) & $\begin{array}{l}\text { The Measurement of Ecological Attitudes and } \\
\text { Knowledge Revised (MEAK-R) scale }\end{array}$ \\
\hline $\begin{array}{l}\text { Stern, Dietz, \& Guagnano, } \\
\text { (1998) }\end{array}$ & USA & General population & 420 & Schwartz Value Survey (Schwartz, 1992; 1994) & Pro-environmental behaviour and willingness \\
\hline Takahashi, \& Zhang (2001) & Japan & & 600 & Environmental values (Branzei et al., 2001) & $\begin{array}{l}\text { Environmental attitudes and environmental } \\
\text { training (behaviour) }\end{array}$ \\
\hline
\end{tabular}


Table 3.2

Descriptive Statistics of Samples

\begin{tabular}{|c|c|c|c|c|c|c|c|c|}
\hline Variable & $\%$ & Min & Max & $M$ & $S D$ & $N$ & $k$ & Not stated $(k)$ \\
\hline Sample size $(n)$ & - & 13 & 2338 & - & - & 47660 & 90 & - \\
\hline Age (mean) & 48 & 15 & 55 & 29.70 & 10.44 & 11097 & 43 & 47 \\
\hline Gender & & & & & & & & 44 \\
\hline Female & 56 & - & - & - & - & 6235 & 46 & - \\
\hline Male & 44 & - & - & - & - & 4953 & 46 & - \\
\hline \multicolumn{9}{|l|}{ Sample type } \\
\hline General population & 61 & 13 & 2338 & - & - & 40536 & 55 & - \\
\hline University students & 39 & 78 & 520 & - & - & 7124 & 35 & - \\
\hline \multicolumn{9}{|l|}{ Type of value's measure } \\
\hline Schwartz's values measure & 54.4 & - & - & - & - & 12082 & 49 & - \\
\hline Inglehart's values measure & 28.9 & - & - & - & - & 28086 & 26 & - \\
\hline Other values measures & 16.7 & - & - & - & - & 7492 & 15 & - \\
\hline \multicolumn{9}{|l|}{ Type of environmental measure } \\
\hline Attitudes & 29.45 & - & - & - & - & 9952 & 43 & - \\
\hline Behaviours & 34.25 & - & - & - & - & 13726 & 50 & - \\
\hline Concern & 23.97 & - & - & - & - & 33157 & 35 & - \\
\hline Willingness & 12.33 & - & - & - & - & 4088 & 18 & - \\
\hline
\end{tabular}

Note. $k=$ number of independent samples

As can be seen in Table 3.1, the year of data collection of the studies ranged from 1994 to 2011. Furthermore, all studies were published in English. According to Table 3.2, gender was not reported for $49 \%$ of the 90 samples. For those that provided this information, $56 \%$ were female. Data on age was missing for $52 \%$ of the 90 samples. For those studies where this information was reported, the age of participants ranged from 15 to $55(M=30.00 ; S D=10.44)$. Samples were coded into the categories of students (39\%) and general population (61\%). The proportion of countries represented in the final dataset was $45 \%$ from Europe, $14 \%$ from North America, 13\% from South America, 11\% from Oceania, 8\% from Asia, 6\% from Central America and the Caribbean, 1\% from Africa, 1\% from the Middle East, and 1\% did not state the country where the sample came from (see Table 3.3 below). 
Table 3.3

Number of Samples and Subjects by Country and Region of the World

\begin{tabular}{|c|c|c|c|}
\hline Countries and world regions & Samples & Subjects & $\%$ of total \\
\hline Europe & 40 & 26267 & 45 \\
\hline Bulgaria & 1 & 1036 & \\
\hline Czech Republic & 2 & 1070 & \\
\hline Estonia & 1 & 400 & \\
\hline France & 2 & 353 & \\
\hline Germany & 3 & 2136 & \\
\hline Hungary & 1 & 1112 & \\
\hline Ireland & 2 & 1620 & \\
\hline Italy & 2 & 1474 & \\
\hline Latvia & 1 & 407 & \\
\hline Netherlands & 6 & 3144 & \\
\hline Norway & 1 & 1224 & \\
\hline Poland & 1 & 1440 & \\
\hline Russia & 2 & 1810 & \\
\hline Slovenia & 1 & 830 & \\
\hline Spain & 3 & 1431 & \\
\hline Sweden & 6 & 3573 & \\
\hline Switzerland & 1 & 445 & \\
\hline Turkey & 1 & 1565 & \\
\hline United Kingdom & 3 & 1197 & \\
\hline North America & 13 & 5211 & 14 \\
\hline Canada & 4 & 2129 & \\
\hline Mexico & 1 & 187 & \\
\hline United States & 8 & 2895 & \\
\hline South America & 12 & 2406 & 13 \\
\hline Colombia & 1 & 149 & \\
\hline Ecuador & 1 & 201 & \\
\hline Paraguay & 1 & 200 & \\
\hline Venezuela & 1 & 194 & \\
\hline Brazil & 6 & 1278 & \\
\hline Peru & 2 & 384 & \\
\hline Oceania & 10 & 7472 & 11 \\
\hline Australia & 6 & 5678 & \\
\hline New Zealand & 4 & 1794 & \\
\hline Asia & 7 & 4129 & 8 \\
\hline China & 3 & 950 & \\
\hline India & 1 & 210 & \\
\hline Japan & 2 & 1782 & \\
\hline Philippines & 1 & 1187 & \\
\hline Central America and Caribbean & 5 & 706 & 6 \\
\hline Costa Rica & 1 & 213 & \\
\hline El Salvador & 1 & 194 & \\
\hline Panama & 1 & 100 & \\
\hline Dominican Republic & 1 & 121 & \\
\hline Nicaragua & 1 & 78 & \\
\hline Middle East & 1 & 1060 & 1 \\
\hline Israel & 1 & 1060 & \\
\hline Africa & 1 & 257 & 1 \\
\hline South Africa & 1 & 257 & \\
\hline Not stated & 1 & 152 & 1 \\
\hline TOTAL & 90 & 47660 & $100 \%$ \\
\hline
\end{tabular}




\section{Effect Sizes}

The correlation coefficients of the studies included in the meta-analysis were transformed using Fisher's $r$ to $z$ transformation (Hedges \& Olkin, 1985). This procedure allowed for a simpler calculation of weights (Fischer, 2013) and research has shown that this transformation minimises Type I error rates (Alexander, Scozzaro, \& Borodkin, 1989). The correlation coefficients were then weighted by sample size $(N-3)$ to provide a more accurate estimate of the corresponding population value (Lipsey \& Wilson, 2001). Using the weighted correlations, an average effect size per study was then calculated between values and environmental engagement (attitudes, behaviours, concern, and willingness). The effect size is based on random effects assuming that effect sizes are not drawn from the same population of studies (Lipsey \& Wilson, 2001). Mean effect size is reported ( $r$ ) with confidence intervals (CI), and a homogeneity test (Q) was applied to the effect sizes.

\section{Moderator Analyses}

A benefit of a meta-analysis is that it enables the consideration of potential moderators that might be difficult to assess within a single study. Identifying moderator variables is important, as doing so helps determine the conditions under which particular values dimensions have most influence on people's environmental acts and could thus inform possible interventions. As mentioned before, the observed strength of the associations between values and environmental attitudes and behaviours and the ways these variables have been measured varies across studies. Thus we coded each study to investigate the influence of these methodological differences and any unexplained variance in the relationship between values and environmental engagement. In the present meta-analysis three variables were considered as potential moderators that could influence the relationship between values and environmental engagement, i.e., sample type, type of values measure, and type of environmental outcomes.

The sample type refers to the type of population from which the sample is drawn. This constitutes an interesting moderator, as psychological research often relies on student samples because this population is easier to find and is more accessible (Henrich, Heine, \& Norenzayan, 2010). However, Peterson (2001) argues that student samples are often more homogeneous than 
samples from the general population and often the effect sizes differ in size and direction between student and general population samples.

Another study-level moderator is the type of values measure. This moderator provides some insight into what are the best measures to assess values in association with environmental engagement. If values have been assessed over time and shown to predict environmental engagement, it is likely that a diverse range of values measures have been used over the years, with some more popular than others. For example, Schwartz's values measure has been used to examine associations with a large number of variables, such as environmental attitudes (Boer \& Fischer, 2013). The challenge is to identify what are the most suitable values measures to explain environmental engagement.

The final moderator of interest is the type of environmental outcomes. This refers to whether environmental attitudes, behaviour, willingness or concern were assessed and which of these are more strongly associated with values. As previously noted, studies have shown that values influence attitudes, which then influence behaviour and there is a stronger association between values and attitudes than between values and behaviours (e.g., Ajzen, 1991). However, other studies have found that the association between values and behaviours is more direct than through the joint association with attitudes (e.g., Hurst et al., 2013). Furthermore, the strength of the association between values and other psychological measures, such as concern and willingness, may also differ considerably from the strength of the association between values, attitudes and behaviours. By establishing the strength of the association between values and attitudes, behaviours, concern and willingness, it is possible to consider the multiple ways in which values may be linked with different environmental outcomes.

The decision to conduct a moderator analysis with these three moderators was based on three main reasons: 1) Most of the meta-analyses reported in the literature (Boer \& Fischer, 2013; Hurst et al., 2013; Milfont et al., 2012) overlap in using the three moderators described in this study, which suggests these moderators are important; 2) All studies included in the present meta-analysis reported these three basic types of information, while other information was not available in all studies (such as gender and Cronbach's alpha); and 3) Including these moderators allows the current study to make a valuable contribution because they can help to identify the best measures to examine the associations between values and environmental engagement. 
In summary, focusing on these three moderators helps to determine whether the strength of the relationship between values and environmental engagement varies particularly in relation to the participants' grouping (students and general population), the values measure (Schwartz and others' measures) or the type of environmental act (i.e., attitudes, behaviours, concern, or willingness). More precisely, sample type was coded with 0 for students and 1 for general population, values measure was coded with 0 for the Schwartz's measure and 1 for other values measures, and the environmental measure was coded with 0 for attitudes, 1 for behaviours, 2 for concern, and 3 for willingness. The moderator analysis examined the heterogeneity of the overall effect size estimates and searched if these moderators explained any heterogeneity. For the moderator analysis, mean random effect sizes ${ }^{5}$ are reported $(r s)$ with confidence intervals (CI), which were tested with between-class homogeneity $\left(\mathrm{Q}_{\mathrm{B}}\right)$ and within-class homogeneity $\left(\mathrm{Q}_{\mathrm{w}}\right)$.

\section{Results}

\section{Overall Effect Sizes}

Individual samples with fewer than 10 participants were excluded from the analysis. For this reason, the final data set comprised a total of 90 individual samples. Participants' scores were checked regarding outliers using stem-and-leaf plots. Two cases of outliers were found for the relationships between ST values and environmental behaviours, and between SE values and environmental behaviours. The two extreme outlier cases were excluded from the final analysis.

Results described in Table 3.4 revealed that a significant positive small effect was found between ST values and environmental behaviours $r(k=39)=.19$, 95\% CI [.17, .21]; Q(38) = $157.40, p<.001$. A significant (albeit weaker) negative effect was also found between $\mathrm{SE}$ values and environmental behaviours, $r(k=37)=-.04,[-.06,-.02]$; $\mathrm{Q}(36)=145.28, p<.001$. Similar results were observed for environmental attitudes, with a significant positive medium effect between ST values and environmental attitudes, $r(k=28)=.24,[.21, .26]$; $\mathrm{Q}(27)=139.10, p$ $<.001$, and a significant negative weaker effect between SE values and environmental attitudes, $r(k=24)=-.13,[-.16, .10] ; \mathrm{Q}(23)=81.51, p<.001$.

\footnotetext{
${ }^{5}$ Mean fixed effect sizes were also calculated and yielded similar results as the mean random effect sizes.
} 
Table 3.4

Summary of Effect Sizes for Values and Environmental Engagement

\begin{tabular}{|c|c|c|c|c|c|c|}
\hline Variable & $R$ & $k$ & $N$ & $\begin{array}{c}95 \% \text { CI } \\
\text { (Lower-Upper) }\end{array}$ & $\begin{array}{c}\mathrm{Q}_{\mathrm{B}} \\
\text { (sample type) }\end{array}$ & $\begin{array}{c}\text { Qв }_{\text {в }} \text { type of values } \\
\text { measure) }\end{array}$ \\
\hline \multicolumn{7}{|l|}{ Environmental behaviours } \\
\hline Self-transcendence values & $.19 * * *$ & 41 & 8082 & .17 to .21 & $4.28 * *$ & $5.03 *$ \\
\hline Self-enhancement values & $-.04 * * *$ & 38 & 7737 & -.06 to -.02 & $20 * * *$ & $6.03 * *$ \\
\hline \multicolumn{7}{|l|}{ Environmental attitudes } \\
\hline Self-transcendence values & $.24 * * *$ & 28 & 5336 & .21 to .26 & $2.27 *$ & $4.47^{*}$ \\
\hline Self-enhancement values & $-.13^{* * *}$ & 24 & 4945 & -.16 to .10 & $25.10 * * *$ & 0.04 \\
\hline \multicolumn{7}{|l|}{ Environmental concern } \\
\hline Self-transcendence values & $.20 * *$ & 3 & 1388 & .14 to .25 & $4.77^{*}$ & $10.36^{* *}$ \\
\hline Self-enhancement values & $.09 * * *$ & 2 & 855 & .03 to .16 & - & - \\
\hline \multicolumn{7}{|l|}{ Environmental willingness } \\
\hline Self-transcendence values & $.53 * *$ & 2 & 537 & .44 to .61 & $6.94 * *$ & $6.94 * * *$ \\
\hline Self-enhancement values & - & 1 & 420 & - & - & - \\
\hline
\end{tabular}

Note. $r=$ average Pearson correlation; $k=$ number of samples in analysis; $N=$ total number of participants; $\mathrm{CI}=$ confidence interval; and $\mathrm{Q}_{\mathrm{B}}=$ between class homogeneity.

$* p<.05, * * p<.01, * * * p<.001$ 
Similar results were observed between ST and both environmental concern and willingness. A significant positive medium effect was found between ST values and environmental concern, $r(k=3)=.20,[.14, .25] ; \mathrm{Q}(2)=10.74, p<.01$ and a significant positive strong effect was found between ST values and willingness, $r(k=2)=.53[.44, .61] ; \mathrm{Q}(1)=$ $6.94, p<.01$. Surprisingly, a positive and significant trivial effect was found between SE values and environmental concern, $r(k=2)=.09[.03, .16] ; \mathrm{Q}(1)=14.94, p<.001$. In addition, there were not enough cases to run an analysis to check for the effect of SE values on environmental willingness (i.e., only one study/effect size was reported for this relationship).

Although the effect sizes visually differ in terms of strength between different types of environmental engagement, there was an overlap of effect sizes and the confidence interval for associations between ST values and environmental attitudes, behaviours, concern and willingness. This indicates that there is no difference in terms of strength of the associations between ST values and these types of environmental outcomes. On the other hand, there was no overlap between effect sizes and confidence interval for the associations between SE values and environmental attitudes, behaviours and concern. This indicates a difference of strength for associations between SE values and environmental outcomes, where the relationship between SE values and environmental attitudes is stronger compared to the association between SE values and environmental concern, followed by the association between SE values and environmental behaviours.

In summary, these results confirmed the prediction of a significant and positive association between ST values and environmental engagement (attitudes, behaviours, concern and willingness). The results also supported the hypothesis that a significant and negative association would be found between SE values and environmental engagement (mainly, environmental attitudes and behaviours). It was also predicted that stronger correlations would be found between ST/SE values and environmental attitudes, willingness and/or concern than with environmental behaviours. This hypothesis was only partially supported. The strength of the associations between ST values and the different types of environmental outcomes did not differ. However, SE values are in general more strongly associated with attitudes than with concern and behaviours. In conclusion, the strength of associations between values and different environmental outcomes depends on the value dimension investigated. 


\section{Moderators}

Heterogeneous effect sizes were found for the correlations between values dimensions (ST and SE) and environmental engagement (attitudes, behaviours, concern and willingness) (see Table 3.4). These findings indicate the existence of additional variability in the effect sizes. Moderator analyses were then conducted to examine whether the characteristics of the studies, such as sample type, type of values measure, and type of environmental outcomes, were significantly related to effect size variability.

\section{Sample Type}

Previous literature has reported that young people with higher education are more prone to act in a pro-environmental way (Fransson \& Gärling, 1999; Stern et al., 1998). Because of their younger average age and potentially more liberal ideals (Milfont et al., 2012), it would be expected that students may be more prone to change values and, consequently, more likely to engage in pro-environmental actions than the general population (Diamantopoulosa, Schlegelmilch, Sinkovics, \& Bohlen, 2003; Hawcroft \& Milfont, 2010). Thus, the first moderator analysis of this study involved the sample type, comparing students (pupils and university students) and the general population (including people from various locations, and also a specific sample of farmers and employees in non-governmental organizations).

The moderator analyses based on the sample type first focused on the relationships between ST/SE values and environmental behaviours. Sample type was found to moderate both of these relationships, $\mathrm{Q}_{\mathrm{B}}(1)=4.28, p<.05$ and $\mathrm{Q}_{\mathrm{B}}(1)=20, p<.001$, respectively. For the relationship between ST values and environmental behaviour, mean effect sizes were larger for studies using general population samples than those studies using student samples $(r \mathrm{~s}=.22$ and .17, respectively). However, the $95 \%$ CI for the effect size for general population samples $(.19, .25)$ did overlap with those for student samples $(.14, .20)$, suggesting that although there was a significant moderation effect of sample type for the association between ST values and environmental behaviour, this association is similar across student and population samples. For the relationship between SE values and environmental behaviour, in contrast, mean effect sizes were larger for studies using student samples than those studies using general population samples ( $r \mathrm{~s}=-.08$ and .02 , respectively). Indeed, the $95 \% \mathrm{CI}$ for the effect size for students sample (-.11, 
-.06) did not overlap with those for general population samples (i. e., -.02, .06) indicating that the relationship between SE values and environmental behaviour was stronger for student samples. It is also interesting to note that the average effect size for the associations between SE values and environmental behaviour was positive (.02, albeit non-significantly) for general population samples.

The moderator analyses based on the sample type (student or general population) were then examined for the relationships between ST and SE values and environmental attitudes. Sample type did not moderate the relationship between ST values and environmental attitudes, $\mathrm{Q}_{\mathrm{B}}(1)=2.27, p>.05$, but it did moderate the relationship between SE values and environmental attitudes, $\mathrm{Q}_{\mathrm{B}}(1)=25.10, p<.001$. For the relationship between $\mathrm{SE}$ values and environmental attitudes, mean effect sizes were larger for studies using student samples than those studies using general population samples ( $r \mathrm{~s}=-.16$ and .02 , respectively). The $95 \% \mathrm{CI}$ for the effect size for student samples $(-.19,-.13)$ did not overlap with the $95 \%$ CI for the effect size for general population samples $(-.04, .08)$. This finding indicates that the association between SE values and environmental attitudes were stronger for student samples compared to general population samples. Again, it is interesting to note that the average effect size for the associations between SE and environmental attitudes was positive (.02, albeit non-significantly) for general population samples.

Sample type also moderated the relationship between ST values and environmental concern, $\mathrm{Q}_{\mathrm{B}}(1)=4.77, p<.05$. Mean effect sizes were larger for studies using student samples than those studies using general population samples ( $r \mathrm{~s}=.28$ and .15 , respectively). However, the $95 \% \mathrm{CI}$ for the effect size for students samples $(.19, .37)$ overlapped with those for general population samples (i.e., .09, .22), meaning that although there was a significant moderation effect, the association between ST and environmental concern is similar across student and population samples. There were not enough cases to run moderation analyses for the relationship between SE values and environmental concern moderated by sample type (only two independent studies both using general population samples).

Furthermore, sample type also moderated the relationship between ST values and environmental willingness, $\mathrm{Q}_{\mathrm{B}}(1)=6.94, p<.01$. Mean effect sizes were larger for studies using general population samples than those studies using student samples $(r \mathrm{~s}=.59$ and .31 , 
respectively). The $95 \% \mathrm{CI}$ for the effect size for general population $(.49, .68)$ overlapped with those for student samples (i.e., .13, .50). This indicates that although a significant moderation was found, the association between ST values and environmental willingness is practically the same across student and general population samples. As mentioned previously, there were not enough cases to run analyses to check for the overall effect of SE values on environmental willingness (i.e., only one study/effect size was reported for this relationship), consequently, there were not enough cases to check the moderation effect of SE values on environmental willingness.

In summary, moderator analyses showed that sample type influences the correlations between ST values and environmental behaviours, concern and willingness, as well as the correlations between SE values and environmental behaviours and attitudes. Overall, correlations between values and environmental outcomes were stronger for student samples compared to general population samples. Although this moderation effect was statistically significant, confidence intervals of the effect sizes for the sample types tended to overlap for ST values.

\section{Type of Values Measure}

The second moderator analysis of this study included the type of values measure, comparing Schwartz's values measure and other values measures (including the Portrait Value Questionnaire, the Brief Inventory of Values, the Stern's Environmental Values measure, the Rokeach Values Survey, the Quality of Life Indicators, the Value Orientations, the AllportLindzey Study of Values, and the Inglehart's Post-materialistic Values measure). Because different types of values measures have been used in the literature to assess values (see for example, Inglehart, 1990; Rokeach, 1973; Schwartz, 1992), it was expected that these different types of values measures would moderate the relationship between values dimensions and environmental outcomes.

The moderator analyses based on the type of values measure first focused on the relationships between ST/SE values and environmental behaviours. Specific types of values measures significantly moderated the relationship between ST values and environmental behaviours, $\mathrm{Q}_{\mathrm{B}}(1)=5.03, p<.05$, and between $\mathrm{SE}$ values and environmental behaviours, $\mathrm{Q}_{\mathrm{B}}(1)$ $=6.03, p<.01$. For the relationship between ST values and environmental behaviour, mean 
effect sizes were larger for studies using the original Schwartz's measure (i.e., SVS, and PVQ) than those studies using other measures of values (e.g., Inglehart's values measure, and Stern's value measure) ( $r \mathrm{~s}=.20$ and .13 , respectively). However, for the relationship between SE values and environmental behaviours, the use of other measures of values showed larger (although positive) effect sizes than the use of Schwartz's scale ( $r$ s $=.15$ and -.05, respectively). Also, for the relationship between ST values and environmental behaviours, the 95\% CI for studies using Schwartz's scale (i.e., .18, .23) overlapped with the 95\% CI for studies using other scales (i.e., .08, .19). However, for the relationship between $\mathrm{SE}$ values and environmental behaviour, the $95 \%$ CI for studies using Schwartz's scale (i.e., -.06, -.02, respectively) did not overlap with the $95 \% \mathrm{CI}$ for studies using other scales (i. e., -.01, .30). This indicates that the relationships between ST values and environmental behaviours are similar across different value measures, while there are different associations between SE values and environmental behaviours depending on the type of values measure (Schwartz or others' scales).

The moderator analyses based on the type of values measure were then examined for the relationships between ST and SE values and environmental attitudes. Significant results were found for the relationship between ST values and environmental attitudes, $\mathrm{Q}_{\mathrm{B}}(1)=4.47, p<.05$, where the Schwartz's measure showed a larger effect size than the other measures of values $(r \mathrm{~s}$ $=.25$ and .18 , respectively). However, the 95\% CIs overlapped (i.e., .22, .28 for the Schwartz's measure and $.13, .24$ for other measures). On the other hand, the type of values measure did not moderate the relationship between SE values and environmental attitudes, $\mathrm{Q}_{\mathrm{B}}(1)=.04, p>.05$. These results indicated that although there is a significant moderator effect between ST values and environmental attitudes, this association is similar across studies using values measures, and that type of values measure does not moderate the associations between SE values and environmental attitudes.

The results also suggested a moderation effect of type of value measure for the association between $\mathrm{ST}$ values and environmental concern, $\mathrm{Q}_{\mathrm{B}}(1)=10.36, p<.01$. This specific relationship was larger with other measures of values than with the Schwartz's measure ( $r \mathrm{~s}=.26$ and .07 , respectively), with no overlap for the $95 \%$ CI for other measures (i.e., .19, .32) and Schwartz's measure (i.e., -.02, .17). This means that associations between ST values and environmental concern were stronger for other measures of values compared to the Schwartz's 
measure. There were not enough cases to proceed with an analysis for moderation effects of types of values in the association between $\mathrm{SE}$ values and environmental concern (only two independent studies, both using the Schwartz's values measure).

A significant moderation effect for the type of values measure was also observed for the relationship between $\mathrm{ST}$ values and willingness, $\mathrm{Q}_{\mathrm{B}}(1)=6.94, p<.001$, although there were only two studies that assessed these variables. The Schwartz's measure showed larger effect sizes than the other values measures ( $r \mathrm{~s}=.59$ and .31 , respectively), and the $95 \%$ CIs did not overlap (i.e., .49, .68 and .13, .49, respectively), indicating that the relationship between ST values and environmental willingness is stronger for studies using Schwartz's measure of values compared to studies using other measures to assess values. There were not enough cases to proceed with an analysis to test the moderation effect of type of values measure for the relationship between willingness and SE values (only 1 independent study using Schwartz's values measure).

In summary, the moderator analyses showed that the type of values measure influences the correlations between ST values and environmental behaviours, attitudes, concern and willingness, and between SE values and environmental behaviour. However, results also showed that the associations between ST values and environmental outcomes are in general similar across types of values measures (Schwartz and others' measures). The exception being for environmental concern, for which other values measures had a stronger association compared to the Schwartz's values measure, and environmental willingness, for which the Schwartz's measure had a stronger association compared to other values measures. Interestingly, other types of values measures are responsible for a stronger association between SE values and environmental behaviours.

\section{Type of Environmental Outcomes}

Finally, within-class homogeneity $\left(\mathrm{Q}_{\mathrm{w}}\right)$ was calculated to test whether the type of environmental measure moderates the relationships between values dimensions (ST/SE values) and environmental engagement (environmental behaviours, attitudes, concerns and willingness). Because environmental attitudes differ from environmental behaviours (see Ajzen \& Fishbein, 1980; Homer \& Kahle, 1988; Milfont, Duckitt, \& Wagner, 2010), and from environmental 
concern and willingness, it was expected that these different types of environmental outcomes would moderate the relationship between values dimensions and environmental engagement.

Some studies included in the meta-analysis only reported one environmental measure, which was either environmental attitudes, behaviours, concern or willingness. At the same time, other studies measured two or more of these environmental outcomes. Most studies only assessed one single environmental outcome (57\%; 51 studies), followed by studies that assessed two environmental outcomes $(29 \%$; 26 studies). Ten studies $(11 \%)$ assessed three environmental outcomes, and only three studies (3\%) assessed all four environmental outcomes. Due to the small number of studies assessing four environmental outcomes, moderation analyses for the type of environmental outcomes focused only on studies assessing one environmental outcome or a combination of two or three outcomes. Following this, each moderation effect was tested for each set of dependent variables separately (i.e., studies assessing only one environmental outcome, studies assessing two environmental outcomes, and studies assessing three environmental outcomes) ${ }^{6}$.

\section{Studies with only one environmental outcome}

For studies assessing only one environmental outcome, the results showed significant Qw between ST values and environmental behaviours, $\mathrm{Qw}_{\mathrm{w}}(39)=138.23, p<.001$, SE values and environmental behaviours, $\mathrm{Q}_{\mathrm{w}}(36)=157.47, p<.001$, ST values and environmental attitudes, $\mathrm{Qw}_{\mathrm{w}}(26)=134.24, p<.001$, and SE values and environmental attitudes, $\mathrm{Qw}_{\mathrm{w}}(22)=57.11, p$ $<.001$. The $\mathrm{Qw}$ value was not significant for the relationship between ST values and environmental concern, $\mathrm{Q}_{\mathrm{W}}(1)=5.97, p<.05$. An analysis could not be performed to test the moderation effect of types of environmental measures for the relationship between SE values and environmental concern, and for the relationships between ST/SE values and environmental willingness. This was because of the limited numbers of studies that provided correlations for

\footnotetext{
${ }^{6}$ Note that there is a distinction between the number of independent samples and the number of overall effect sizes considered. There were only 90 independent samples for the meta-analysis and each study was only counted once. However, one particular independent sample could have produced from one to four effect sizes, depending on the number of environmental outcomes measured.
} 
these relationships (two studies assessed both SE values and environmental concern; two studies assessed ST values and environmental willingness; and only one study assessed both SE values and environmental willingness).

Confidence intervals for the effect size of the association between ST values and environmental behaviours $(.17, .22)$ overlapped with those for environmental attitudes $(.21, .26)$, indicating that although the associations for ST values are stronger for environmental attitudes than for environmental behaviours, they are similar across the different types of environmental measures. For SE values, the $95 \%$ CI for environmental behaviours (-.07, -.02) did not overlap with those for environmental attitudes (-.16, -.10), indicating that the association between SE values and attitudes is stronger than the association between SE values and environmental behaviours.

\section{Studies with two environmental outcomes}

For the studies assessing two environmental outcomes, the results showed a significant moderator effect of the type of environmental outcomes in the association between ST values and environmental behaviours, $\mathrm{Qw}_{\mathrm{w}}(22)=101.29, p<.001$, and environmental attitudes, $\mathrm{Q}_{\mathrm{w}}(22)$ $=107.83, p<.001$. Confidence intervals for the association between ST values and environmental behaviours $(.13, .18)$ were positively weaker and did not overlap with those from the association between ST values and environmental attitudes $(.19, .25)$, indicating that these relationships differ according to the type of environmental outcome assessed. The type of environmental outcome also moderated the relationship between SE values and environmental behaviours, $\mathrm{Q}_{\mathrm{w}}(19)=29.83, p<.05$, and attitudes, $\mathrm{Q}_{\mathrm{w}}(19)=43.21, p<.01$. Moreover, confidence intervals did not overlap and were stronger for the association between SE values and environmental attitudes (-.19, -.13) than between SE values and environmental behaviours (-.06, -.01). This indicates that the type of environmental variable used could explain heterogeneity in effect sizes of the relationship between SE values and environmental behaviours and attitudes.

An analysis could not be performed to test the moderator effect of the type of environmental outcomes for the relationships between ST and SE values and environmental concern and willingness. This was because of limited number of studies that provided correlations for these relationships (all three studies assessed ST values and environmental 
concern; both two studies assessed SE values and environmental concern; and only one study assessed SE values and environmental willingness).

\section{Studies with three environmental outcomes}

For studies with three environmental outcomes, results showed a significant moderation of the type of environmental outcome in the association between ST values and environmental behaviours, $\mathrm{Q}_{\mathrm{W}}(8)=44.13, p<.001$, and attitudes, $\mathrm{Q}_{\mathrm{W}}(8)=45.56, p<.001$. Confidence intervals did not overlap for ST values and environmental attitudes $(.24, .32)$ and were positive and stronger than the confidence intervals between ST values and environmental behaviours $(.12, .20)$, indicating that these relationships differed across the type of environmental outcome assessed. Results were not significant for SE values and environmental behaviours, $\mathrm{Q}_{\mathrm{w}}(8)=$ $8.17, p>.05$, but a significant moderation was found for SE values and environmental attitudes, $\mathrm{Q}_{\mathrm{w}}(8)=29.89, p<.01$, and confidence intervals fell from -.19 to -.11.

An analysis could not be performed to test the moderator effect of the type of environmental outcomes on the associations between ST values and environmental concern and willingness and between SE values and environmental concern and willingness. This was because of the limited number of studies that provided correlation for these relationships (all three studies assessed ST values and environmental concern; both two studies assessed ST values and environmental willingness; both two studies assessed SE values and environmental concern; and only one study assessed SE values and environmental willingness).

In summary, when moderator analyses were performed to check if the type of environmental outcomes affects the association between values and environmental outcomes, generally the results showed that the moderation is significant and the relationship between $\mathrm{ST} / \mathrm{SE}$ values is stronger when the environmental measure assessed environmental attitudes compared to environmental behaviours. Overall, moderator analysis showed that the type of sample, type of values measures, and the type of environmental outcomes explained some of the heterogeneity exhibited by the effect size between value dimensions and environmental outcomes. No other sources of heterogeneity were explored in this study. 


\section{Discussion}

The aim of this study was to provide a quantitative review of the associations between values and environmental engagement. A meta-analytical approach was employed to integrate results of past studies to clarify the main value dimensions associated with attitudes, behaviours, concern and willingness towards the environment. The meta-analysis conducted in this study overcame limitations of previous meta-analyses (Hurst et al., 2013; Boer \& Fischer, 2013) specifically on two aspects: 1) the present meta-analysis assessed a broader range of values theories and measures (i.e., the Schwartz Value Survey, the Portrait Value Questionnaire, the Brief Inventory of Values, the Stern's Environmental Values measure, the Rokeach Values Survey, the Quality of Life Indicators, the Value Orientations, the Allport-Lindzey Study of Values, and the Inglehart's Post-materialistic Values measure); and 2) it assessed a broader range of environmental outcomes (i.e., attitudes, behaviours, concern and willingness).

The present study included 47,660 participants from 41 countries and 90 independent samples, and established that values play an important role in influencing a diverse number of environmentally relevant variables in the two main streams of values dimensions related to environmental engagement (labelled as ST and SE values dimensions). Supporting predictions, more collective values (such as ST values) had the strongest association with environmental engagement and this relationship followed the expected positive direction. The analysis also showed that methodological aspects, such as the type of values measured, affected the association between values and environmental engagement. The implications of these findings are discussed below, and recommendations for future research are made.

\section{On the Associations between Values and Environmental Engagement}

The results suggested that ST values best predict environmental engagement. In other words, the present meta-analysis attested that people who greatly value their relationships with the community, family and peers are also more inclined to care about the environment (Becker $\&$ Félonneau, 2011; Feather, 2002; Fukukawa et al., 2007; Hansla, Gärling, \& Biel 2013; Milfont, Sibley \& Duckitt, 2010; Nordlund \& Garvill, 2002; Schultz et al., 2005; Schultz \& Zelezny, 1999; Stern et al., 1998). As expected, the results of the present study supported previous 
findings that individuals who are oriented by ST values care about the environment and are more inclined to act to address environmental issues (e.g., Boer \& Fischer, 2013).

Overall, the results of the present study are consistent with other meta-analyses. These findings confirm previous research showing that the effect size for ST values in relation to proenvironmental engagement (Boer \& Fischer, 2013) is greater than the effect size observed for SE values and pro-environmental engagement (Hurst et al., 2013). Although the stronger effect of ST values on environmental outcomes is well supported, it is important to note that most of the studies in our meta-analysis did not report relationships between SE values and environmental outcomes. It is possible that a stronger, more consistent negative effect for SE values would have been observed in the current analyses if more researchers included these in their own publications. To address this issue, the associations between environmental outcomes and SE values should be more consistently investigated in future research.

It is also worth noting that the Schwartz's values measure includes environmentally related items within the ST dimension ("protecting the environment", "unity with nature", and "a world of beauty"). The inclusion of such items might inflate associations between ST values and pro-environmental engagement due to content overlap. Past research has created 'pure' altruistic values scores by excluding these environmentally related items from the ST dimension (e.g., Milfont, Sibley, \& Duckitt, 2010; Schultz et al., 2005). This means that the stronger effect sizes observed for ST values compared to SE values might also reflect methodological issues that may inflate the correlations between ST values and pro-environmental engagement. The creation of 'pure' altruistic values scores was not possible in the present meta-analysis (and also in the previous meta-analysis by Boer and Fischer, 2013) because in most of the cases we did not have access to all original datasets. However, this issue is addressed in Study 3 of this thesis.

Besides these two methodological points, a theoretical reason for the stronger effect of ST values relative to the small overall effect of SE values might trigger distinct value-based concerns, especially when it involves their influence on environmental engagement. For example, Schultz et al. (2005) claimed that people who prioritise SE values are concerned about environmental problems only if they perceive such problems as directly affecting their private interests. Therefore, it may be the case that in the present study, the small effect of SE values on 
environmental engagement is due to the fact that the participants who were oriented by SE values did not perceive environmental issues as potentially threatening their personal affairs.

Overall, the effect sizes for both ST values and environmental outcomes and SE values and environmental outcomes were small to medium. The strength of the effect sizes are dependent on the link formed by the variables they represent and some variables can yield a stronger link than others. Small effect sizes are not uncommon in psychology and can be practically important (Milfont et al., 2012). For example, in a meta-analysis conducted by Richard, Bond and Stokes-Zoota (2003), it was found that across the studies in social psychology on various topics, effect sizes yielded an average value of .21 and that around $30 \%$ of those studies yielded an effect size of .10 or less. As a result, studies reporting effect sizes (even small ones) contribute to a more complete understanding of the relationships between diverse numbers of variables that are important to psychological research.

In addition, this study has shown that ST values have comparatively stronger relationships with environmental attitudes than with environmental behaviours (and this was not affected by sample type or values measure). This finding is consistent with previous research that has shown that values can better predict attitudes than behaviours (e.g., Milfont, Duckitt \& Wagner, 2010; Schultz \& Zelezny, 1999). Nevertheless, it is still difficult to find a straight path between motivational guides or values and how people act (Ajzen, 1985; Ajzen \& Fishbein, 1980). Despite this, Hurst et al. (2013), for example, argued that by investigating the strength of value association with both attitudes and behaviour, it would be possible to investigate the multiple ways in which values are linked with environmental outcomes.

\section{On the Contextual Effects}

This study also examined possible contextual moderators of the association between values and environmental engagement. Three possible moderators were considered: sample type (students vs. general population), the type of values measure (Schwartz vs. others' measures), and the type of environmental outcome (environmental attitudes vs. environmental behaviours vs. environmental concern vs. environmental willingness).

It was found that sample type moderates the relationships between ST values and environmental behaviours, concern and willingness. Furthermore, sample type also moderated 
the relationships between SE values and environmental behaviours and attitudes. Although the correlations between SE values and environmental attitudes and behaviours differed across student and general population samples, indicating larger effect sizes for studies using student samples, the confidence intervals of the effect sizes for the sample types overlapped for ST values and environmental behaviours, concern and willingness. These results indicate that, contrary to SE values, the association between ST values and the environmental outcomes in general did not differ across student and general population samples. In conclusion, these findings partially supported the prediction that students are more likely to engage in proenvironmental actions than the general population (Diamantopoulosa et al., 2003; Hawcroft \& Milfont, 2010). The results suggested that further factors beyond sample type may be influencing the relationships between values (especially ST values) and environmental outcomes, such as the type of values measure.

Another interesting result that emerged concerns the unexpected positive correlations observed between SE values and environmental engagement (e.g., SE values and environmental behaviours had an effect size of .02 for the general population samples). The small effect sizes and the unexpected directions were found for the general population, which may indicate that the greater heterogeneity of the general population is affecting the correlations (note that the general population includes varied categories of citizens, such as teachers, compared with the more homogeneous sample of students). In contrast, for the student sample, the pattern is consistent and goes in the expected direction. Also, the correlation between SE values and some environmental outcomes was small. For instance, with the general population sample, the correlation between SE values and environmental behaviours was $r=.02$, while the correlation between SE values and environmental attitudes was $r=.08$. This suggests that, although significant, the effect of SE values on certain types of environmental outcomes is weak. However, the positive correlations between SE values and environmental outcomes can be seen as a theoretical implication instead of a limitation. Other articles had reported positive (unexpected) correlations (e.g., Schultz et al., 2005), so there are precedents in the literature for these anomalous results. One possible explanation for the positive relationships between SE values and environmental outcomes is that people motivated by these type of values would be willing to act in a pro-environmental way if they recognise that environmental problems can affect their comfort and welfare (Schultz et al., 2005). 
The type of values measure explained some of the heterogeneity of the effect estimate. The average effect size for studies measuring values from the Schwartz Value Survey (.20) was higher than those using other scales, for example, Inglehart's values measure (.13). One possible explanation for this distinction in effect sizes is content overlap. As noted above, the Schwartz's measure of ST values included environmentally-related value items (i.e., unity with nature, protecting the environment) which would methodologically inflate the correlation between the values dimension and criterion variables related to environmental engagement. This content overlap is not present in the Inglehart measures. Nevertheless, both Schwartz's measure and the other values measures have a significant medium effect on pro-environmental attitudes and behaviours. This indicates that there are good measures of values available to explain environmental outcomes.

It is important to note that the findings did not fully support the prediction that participants would be more likely to engage in environmental actions when assessed by Schwartz's values measure than when assessed by other measures of values. For example, the confidence interval for the relationships between ST values and environmental attitudes and behaviours for Schwartz and other's values measures overlapped, indicating that associations are similar between type of value measures. Additionally, depending on the type of environmental outcome investigated, other values measures accounted for a stronger effect size than the Schwartz's measure. The results suggest that further factors beyond the values measures influenced the relationships between values and environmental outcomes, such as the type of environmental measure.

Furthermore, the results showed that the type of environmental measure also explained some of the heterogeneity in the link between values and environmental engagement. These findings confirm previous research that has noted differences in the strength of the relationship between values and environmental outcomes (e.g., Grunert \& Juhl, 1995; Milfont, Duckitt, \& Wagner, 2010; Schultz \& Zelezny, 1999). In general, a stronger relationship between values and environmental attitudes was found, instead of between values and environmental behaviours. This was true for direct effects between values and environmental engagement and also for moderator effects of type of environmental outcomes in the relationships between values and environmental engagement. Although, these results differ from the study conducted by Lévy- 
Leboyer et al. (1996), they are consistent with the findings of a great deal of the previous work in this field (e.g., Ajzen \& Fishbein, 1980; Schultz et al., 2005; Milfont, Duckitt, \& Wagner, 2010). These results also have implications for environmental psychology theories, specifically because it confirms previous studies that proposes that values better predict attitudes instead of behaviours (Homer \& Kahle, 1988; Maio \& Olson, 1995).

Finally, it is relevant to note that a variety of environmental outcomes were assessed in the articles included in the present meta-analysis. The different labels that environmental engagement was given in the literature reflected not only the variety of environmental outcomes but many times problematic ways in which environmental scales have been used (for example, the NEP scale has been sometimes categorised as an attitudinal scale and sometimes as a motivational scale). This issue does not seem to be unique to the present study, but has been described by Stern (1992) as an "anarchy of measurement" (p. 279), and was also pointed out in previous studies conducted by Dunlap and Jones (2002), and Milfont and Duckitt (2010).

Overall, the present study suggests that it is important to consider contextual factors that might influence the association between values and environmental engagement. While it is positive to note that research on environmental outcomes and values have assessed a broad range of environmental variables, it is important that researchers consistently define accurate measures of environmental outcomes across different studies. This will help not only the examination of the particular outcome of interest but also its association with other variables such as values.

\section{Limitations}

There are general limitations when conducting a meta-analysis (Rosenthal \& DiMatteo, 2001). These limitations include publication and selection bias, and the so-called 'garbage in, garbage out' issue. The publication bias refers to the fact that only studies reporting significant results tend to be published in the scientific literature, and consequently, these are the only ones included in a meta-analysis. For example, Fanelli (2011) discusses the importance of including (and publishing) negative and non-significant results as a premise to guarantee the core idea of impartiality in science. Selection bias refers to the selection of studies and the process of defining the inclusion criteria of the 'relevant' studies to be incorporated into the meta-analysis. Depending on which criteria are used, this process can create a bias in selecting which papers 
will be included or excluded from the meta-analysis. Finally, the 'garbage in, garbage out' issue refers to the variable quality of the independent studies included in the meta-analysis that determine the validity and accuracy of the meta-analytical results that are produced.

To try to avoid or reduce the impact of these issues, all significant and non-significant findings that met the established selection criteria were included in the present meta-analysis. This study also followed best practise in the field in defining specific selection and inclusion criteria. Furthermore, an effort was made to include all known research on the topic (published and unpublished). Only studies that did not provide a clear research design and those for which effect sizes could not be determined were excluded. It can thus be argued that this analysis provides an accurate and valid review of 36 published and unpublished studies (total of 90 samples) on the relationships between values and environmental engagement outcomes.

Nevertheless, it is important to note the lack of necessary information in studies measuring environmental willingness and concern. Most of the research on the topic of values and environmental engagement has assessed mainly environmental attitudes and behaviours. Some research has measured environmental concern, but regarding willingness, little research is available. It is noted that, in the literature in general, the studies assessing environmental attitudes and behaviours come from the field of environmental psychology while the ones investigating concern and willingness originate mostly in environmental sociology (Dunlap \& Jones, 2002).

Another limitation was the non-inclusion of other possible moderators, such as Cronbach's alpha (e.g., Boer \& Fischer, 2013; Hurst et al., 2013). Reliability indexes such as Cronbach's alpha can affect the effect size between two variables. As a result it is important that researchers develop scales with higher reliabilities to guarantee that there are accurate estimates of the size of associations between variables. Hurst et al. (2013) argued that the lower the reliability of a scale, the greater the increase in the size of the correlation when it is corrected for reliability. However, reliability indicators can only be included as a moderator if this information can be extracted from studies. For example, in their meta-analysis, Hawcroft and Milfont (2010) observed that only half of the 139 original studies reported reliability information. Future studies could, for example, examine the extent to which the reliabilities of environmental and values 
measures reliably impact the associations between SE values and pro-environmental engagement.

Publication year is also a potentially interesting moderator that was not included in the present meta-analysis and could have provided interesting insights into how environmental views varied over the years. Because the main goal of the current study was to consider the overall trend in relationships between values and environmental outcomes, including publication year as a moderating variable was outside of the scope of the current investigation. It is therefore recommended that in future studies, researchers should consider that the relationship between values and environmental outcomes may be impacted by the time and contextual circumstances in which they are embedded.

Another limitation of the present meta-analysis is the non-inclusion of study quality as a potential moderator of the relationship between values and environmental engagement. Although the meta-analysis followed strict inclusion criteria that set minimum levels for inclusion of the articles, it did not directly examine the quality of these studies as a moderator. This is because several criteria of study quality noted in the literature (such as double blind designs) do not apply to this field of research (Conn \& Rantz, 2003). Furthermore, this study focused on moderation analyses (e.g., study sample and type of values measure) that are more theoretically relevant to the research topic. Again, future meta-analyses in the environmental psychology domain could consider other moderators including study quality.

In sum, the limitations of this meta-analysis partially reflect the limitations of the literature available. Some studies did not report important information that would have been valuable for this analysis. Additionally, due to the correlational nature of the research, it is difficult to draw any firm conclusions about causality from the mean effect sizes. The results do, however, present a clear picture of the associations between values and environmental outcomes in the countries from which the samples were drawn. Taken together, these results suggest that indeed there is an association between values and environmental outcomes, and more specifically, that ST values positively and strongly predict environmental engagement while SE values in general, negatively and weakly predicted environmental engagement. 


\section{Directions for Future Research}

Although the findings suggest that ST and SE values are good predictors of environmental engagement as demonstrated by the significant effects of these two values dimensions on environmental engagement, it would be important to also determine the effect of the other two dimensions of Schwartz's values (openness to change and conformity) on environmental outcomes. A study conducted by Nordlund and Westin (2013) has shown that, besides ST vs. SE values, the openness to change vs. conservation dimension of values also explained environmental concern and general travel mode beliefs. According to their findings, people who are motivated by openness to change values are guided by a sense of independent action and readiness for new experiences are more inclined to care about the environment. This effect is likely not in the same direction and with the same strength as the relations with the ST vs. SE values dimension. Still, openness to change values might influence people's environmental engagement, or at least some specific environmental acts associated with trying new things, including not only new general experiences in life but also the feelings of being innovative for using, for example, green energy, solar power and public transport instead of a car. Future research could explore the openness to change vs. conservation values dimension and assess its combined influence on environmental engagement.

Furthermore, future research could look at a higher level analysis of the country differences in the strength of the association between values and environmental engagement considering socio-economic indices. This could suggest evidence about the traditional idea predominant in Western cultures that personal and national economic growth is at odds with protecting the environment. Previous research has found that citizens in nations with lower GDP (Gross Domestic Product) and HDI (Human Development Index) have a lower level of willingness to make sacrifices for the environment (Haller \& Hadler, 2008; Inglehart, 1990). However, in the meta-analysis conducted by Hurst et al. (2013), countries such as Chile, with low a GDP and HDI, did not show negative correlations between materialism and environmental attitudes. Further investigation of these contradictory results would be useful in shedding light on this issue.

Last but not least, the high numbers of studies on the topic of values and environmental engagement that have used self-report measures are very important, but there is a clear need for 
studies to use experimental designs to assess whether priming certain types of values leads to greater environmental engagement. These experimental studies can test the utility of using values to change people's behaviours and attitudes (and perhaps concern and willingness) towards the environment. This issue is addressed in the next set of studies in this thesis.

\section{Conclusion and Practical Applications}

The concern with identifying what values to focus on to make people more environmentally friendly is not new (Hines, Hungeford, \& Tomera, 1986/1987). This study has shown that it might be effective to promote ST values instead of other types of values to promote environmentally friendly endeavours. Communications and environmental campaigns should therefore reinforce ST values, as they are the most likely variable to promote environmental engagement. Additionally, a focus on claims that show how environmentally friendly acts can contribute socially, locally, and globally may be more effective in fostering environmental engagement (Schultz, 2011), instead of focusing on strategies that use punishment and rewards (e.g., higher bills for more energy consumption combined with lower bills and discounts for using less energy).

Furthermore, as promoting specific values (especially ST values) can reinforce environmental engagement, it may be useful to try to change people's values to further encourage environmental outcomes. One of the techniques used to change people's values (and consequently promote environmental engagement) is the VSC technique (Maio et al., 2009). Therefore, the next chapter describes two experimental studies designed to apply this specific technique to change values and make people more environmentally engaged. 


\section{CHAPTER FOUR: STUDY 2}

\section{Experimentally Manipulating Values to Motivate Environmental Behavioural Intentions}

The present chapter describes two experiments that follow from the findings of the metaanalysis (Chapter 3). This set of two experiments aims to address the core question: Can we change people's values and if so, can this change in values influence people's environmental behavioural intentions?

Past research has shown that there is some association between values, attitudes and behaviours (e.g., see meta-analysis described in Chapter 3 of this thesis), and the literature on environmental engagement specifically highlights the importance of ST values for promoting pro-environmental outcomes (Crompton, 2010; Welzel, Inglehart, \& Klingemann, 2003). Most of these studies assess the relationships between values and environmental outcomes using correlational designs (e.g., Schultz et al., 2005), and only a few experiments have been conducted focusing on modifying values (Maio et al., 2009). The majority of the research showing that values can be experimentally manipulated has used the VSC technique (e.g., Maio \& Olson, 1995; Maio et al., 2009; Rokeach, 1973). The present experiments follow this research paradigm by implementing VSC technique to promote value change and assess its impact on environmental behavioural intentions.

\section{The Value Self-Confrontation (VSC) Technique}

The first and perhaps the most well-known researcher to use the VSC technique was Rokeach (1973). As discussed in Chapter 2, Rokeach (1973) argues that values are organised in a hierarchical system from the most to the least important. Additionally, Rokeach (1973) also suggests that when a subset of values is activated in a specific situation, the values that are perceived as relevant to the salient actions may favour various behaviours, or oppose them. For example, valuing equality might favour donating to charity and oppose purchasing an expensive item, whereas treasuring a comfortable life might have the opposite influence. Furthermore, the strength of the impact of people's values on their behaviour depends on the importance of the value in the person's hierarchy. This idea further implies that values of little absolute importance to a person - or those low in the person's importance hierarchy - will have little or no impact, 
leaving it to the more important values to determine a particular behaviour(s). The VSC technique is based on the premise that under ordinary circumstances, people are unaware of their value hierarchy.

Rokeach (1973) proposes four assumptions when describing the VSC technique: 1) the VSC manipulation must increase the target behaviour; 2) the manipulation must change the importance ranking of the target values toward the value priorities of the positive reference group; 3) value priorities like those of the positive reference group must predict the target behaviour; and 4) controlling for the relative importance of the target values must substantially reduce the association between the VSC technique and behaviour. According to Rokeach (1973), people only become aware of their values hierarchy when they need to rank the values in terms of their importance as guiding principles in their lives. Given this premise, the VSC technique is conducted in two basic steps. The first step consists of individuals receiving manipulated feedback emphasizing specific values that participants did not list as value priorities in their lives. The second step is to inform people of value priorities that discriminate between them and their reference groups. At the end of the second step, individuals would be dissatisfied with the discrepancies between their value priorities and what other people prioritise in the manipulated feedback. The dissatisfaction that people feel is caused by self-concepts of competence and morality (Rokeach, 1973).

Values serve to fulfill these self-concepts that are challenged when people are confronted with other people's priorities. This contradiction gives rise to self-dissatisfaction with one's value rankings, creating cognitive or motivational needs to adapt to the values priorities of the norm group. For example, according to Nemeth and Watchtler (1983), one of the possible results of this incongruence between self and others' value priorities is that participants are motivated to reduce the self-dissatisfaction by changing their value priorities and conforming to value priorities of the norm group through a process of social comparison and norm adaptation. The ideal self-conceptions determine the direction of the change. These changes presumably occur in a sequence. First, the importance rankings of values are reordered to resemble more closely those of the positive reference group. Then, value-related attitudes may be modified to be compatible with the values that underlie them. Finally, behaviour changes tend to be consistent with the new values and attitudes. The anchoring of behaviour in the new value system priorities makes the 
behaviour more enduring. In short, the conflict or dissatisfaction between one's value priorities and one's ideal self-conception, usually created by a normative or reference group priority, can generate a favourable setting for value change (Rokeach, 1973).

The VSC technique has become popular among researchers interested in value change and its effect on attitudes and behaviours. The study developed by Maio et al. (2009) is one of the most recent on the topic. It added to previous studies, for instance Rokeach's studies, by showing whether changes in values could be extended to changes in other values, not only the target ones. In a previous study, Maio and Olson (1998) examined how changes in values could affect not only target values but other related values and how these changes reinforce or decrease scores on behavioural measures that affirm those values. In his recent paper, Maio et al. (2009) proposed that, when participants are confronted with relevant people who prioritise different values from their own, they are more inclined to increase the importance placed on these "different" values. Maio et al. (2009) tested the systematic effects of values, using both a manipulation of value change and a manipulation of value priming. They found that experimentally changing some specific values (e.g., ST values: loyalty, equality, and helpfulness) by using the VSC technique increased the importance of values that served the same or similar motives (e.g., ST values: forgiveness, honesty, and social justice) and decreased the importance of values that served opposing motives (e.g., SE values: authority, capable, and influential), see Figure 4.1 below. They also found that priming specific values (e.g., achievement values: success and capability) caused participants to exhibit more behaviours consistent with the primed values (e.g., achievement behaviour), while displaying fewer behaviours (e.g., benevolence behaviour) consistent with the opposed values (e.g., benevolence values: helpfulness and loyalty), see Figure 4.2 below. Consistently across four experiments, the authors found the same pattern of results. The authors concluded that their experiments are the first evidence for systematic change in values and consequent effect on behaviours, providing further affirmation of the compatibilities and conflicts among values of the circular model proposed by Schwartz (1992). 

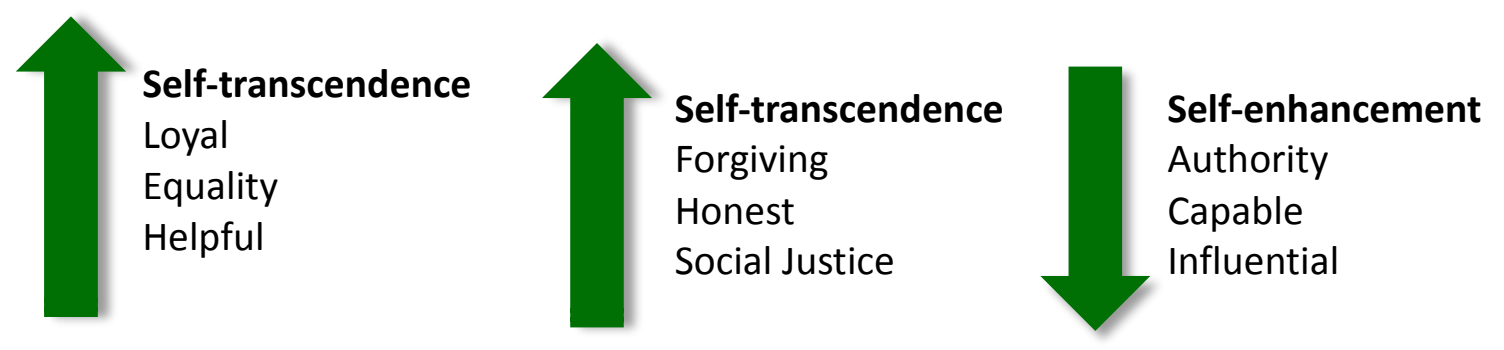

Figure 4.1. Changing some specific values increased the importance of values that served the same or similar motives and decreased the importance of values that served opposing motives Note: Based on results of Study 1 presented by Maio et al. (2009).

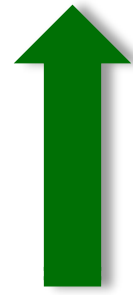

Achievement values (self-enhancement) successful capable

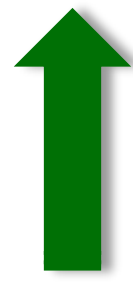

\section{Benevolence values} (self-transcendence) helpful loyal

\section{Achievement behaviour} (word search task)

\section{Benevolence behaviour}

(willing to take part in future research)

\section{Benevolence behaviour}

(willing to take part in future research)

Figure 4.2. Priming specific values caused participants to exhibit more behaviour consistent with the primed values, while less behaviour consistent with the opposed values.

Note: Based on results of studies 2, 3, 4 and 5 presented by Maio et al. (2009).

Although Maio's work forms the cornerstone of the literature on value-change using the VSC technique, the use of self-confrontation methods based on social comparison mechanisms 
to affect behaviour has been employed before in past research (e.g., Chernoff \& Davison, 2005' Schwartz \& Inbar-Saban, 1988). Specifically, an earlier study conducted by Schwartz and InbarSaban (1988) examined the possibility of using the VSC technique to influence behaviour, in this case the weight loss of 87 overweight adults. The authors tested the proposition behind previous research that successful weight losers differ from unsuccessful weight losers because they prioritise values of wisdom more than values of happiness. The results showed that participants exposed to the value self-confrontation condition lost more weight than those exposed to other conditions. In the VSC condition, the reference group demonstrated a behaviour (losing weight) that was desirable to participants, so they were prepared to change their values in order to achieve this result. More importantly, the evidence of weight loss caused by the VSC method persisted over a year. Additionally, the change in values during the period of the first two months suggested that loss of weight was mediated by the increase in the importance attributed to values of wisdom relative to values of happiness. In summary, this study shows a direct effect of the VSC technique on changing a specific behaviour. Furthermore, this effect was shown to persist over the long-term.

The review of the literature summarised above shows that the VSC technique has the capacity to change values hierarchies (e.g., Maio et al., 2009); that is, it changes the ranking of values or the priority given to certain values by the individuals. Because of this characteristic of the VSC technique, the identification of the core values that are relevant for a specific population and that are potentially predictive of a specific behaviour help to determine which values can be targeted and will likely change. The meta-analysis described in the previous chapter has shown that ST values are the best predictors of environmental attitudes and behaviours across different sample types. This provides evidence that ST values can be the target focus to promote relevant environmental outcomes in the experiments described in this chapter.

\section{The Role of Identity}

As discussed in previous chapters, identity is an important variable to take into account when working with social norms (Chernoff \& Davison, 2005). Social comparison with a norm group increases people's willingness to change their values and implies that identity plays an important role in the effectiveness of the VSC technique. As social creatures, humans do not want to be different from those who they feel more connected with (Chen, Wasti, \& Triandis, 
2007) and by comparing themselves to others they seek clues about what is the acceptable way of acting, feeling and thinking (Masters, Carlson, \& Rahe, 1985). The VSC technique involves confronting participants with the value priorities of a reference group by using social comparison to promote subsequent value change with participants conforming to the group norm. People who are highly identified with a reference group tend to be more willing to adapt to the norms of that group, even if that means changing their behaviour, attitudes, concepts, and values (Terry et al., 1999).

Although not greatly emphasised in the specific literature on VSC technique, identity has been shown to be related to values (Hitlin, 2003; Nakashima et al., 2013; Terry et al., 1999). For example, a study conducted by Fritsche, Cohrs, Kessler, and Bauer (2012), showed that relevant values, such as the ST values, are significant predictors of volunteer identity, even when other measures of identity are controlled. Furthermore, research has also shown that identity exerts an influence on environmental outcomes. For example, studies conducted by Fielding et al. (2008) and by Nigbur et al. (2010) showed how self-identity and identity as an environmental group member successfully predicted attitudes towards environmental activism and recycling. Specifically, the study conducted by Fielding et al. (2008) has shown that environmental group membership influences attitudes towards environmental activism. In their study, Fielding and colleagues (2008) found that students who were more involved with environmental groups demonstrated stronger intentions to engage in environmental activism, and participants who had a stronger sense of normative support for environmental activism also had greater intentions to engage in the behaviour. Furthermore, the study conducted by Nigbur et al. (2010) showed that norms can predict residential curbside recycling. In their study, participants showed more willingness to recycle when social norms were activated.

In addition to the already investigated effects of self-identify and identity as an environmental group member focusing on environmental engagement, it can be argued that student identity exerts an effect on environmental outcomes as well. For example, research has shown that students may be more prone to environmental engagement than general populations (Diamantopoulosa, Schlegelmilch, Sinkovics, \& Bohlen, 2003; Hawcroft \& Milfont, 2010). More specifically, Diamantopoulosa et al. (2003) argue that student samples tend to be exposed to higher education and are more likely to undertake recycling activities end engage on green 
political action. Additionally, higher education institutions exert an important impact on society through also fostering the sense of identity among its students (Brennan, King \& Lebeau, 2004). Based on this research, it can be argued that university students would be more likely to participate in green activities than non-students.

In addition to student identity, it can be argued that national identity affects environmental engagement. For example, a qualitative study conducted by Davis and Francis (2014) has unraveled the narratives of children and the results showed that national identity is expressed in environmental and counter-consumption terms. Additionally, national identity has also been formulated and importantly used to frame environmentalism as patriotic - a strategy already used by the pro-environmental lobby (M. S. Wilson, personal communication, September 9, 2014). For example, in an article published in the New York Times, Davenport and Parker (2014) reported that according to an analysis of political advertising in Senate races in the general election in USA in 2014, energy and the environment were amongst the most mentioned subjects in political advertisements, ranking behind health care and jobs.

Based on the literature discussed above, it is suggested that both student identity and national identity can play an important role in the effectiveness of the VSC technique and in the relationship between values and environmental engagement, especially for New Zealand participants. New Zealand is often referred to as a green country (Bührs \& Bartlett, 1993; Memon, 1993). Furthermore, the image of a green New Zealand is promoted by local national advertisements that reinforce the beauty of New Zealand's landscapes and citizens' awareness of environmental acts, such as recycling. It is possible that New Zealand identity is inherently related to these images of New Zealand being green. The two experiments conducted in this thesis will, among other things, explore how identity as a university student and identity as a New Zealander contribute to the effectiveness of the VSC technique to promote value change.

\section{Aims of the Present Study}

The present experiments aim to investigate value change and test its effect on environmental behavioural intentions. In line with initial studies by Rokeach (1973) and later extensions by Maio et al. (2009, Study 1), it is hypothesised that values would change from pretest to post-test. More specifically it is expected that prioritizing ST values (an aspect of the ST 
condition) will increase the importance of other ST values while decreasing the importance of SE values. In contrast, highlighting SE values (an aspect of the SE condition) will increase the importance of other SE values while decreasing the importance of ST values. No change in value priorities is expected for the control condition.

Both experiments described in the present chapter will also explore the role that student and national identity plays as an underlying mechanism in promoting value change. It is expected that people who strongly identify with a given reference group will be more willing to conform to the normative values information of the reference group and to consequently change values priorities. In contrast, it is expected that people who do not strongly identify with the reference group will be less willing to conform to the values prioritised by the reference group, and consequently less willing to change values.

Bearing in mind that behavioural intentions are better predictors of behaviour than attitudes (Ajzen, 1985; Ajzen \& Fishbein, 1980), the present study uses the VSC technique (Maio et al., 2009, Study 1) to manipulate value change and examine the impact of this change on environmental behavioural intentions. Based on previous literature (Maio et al., 2009) that found that reinforcing specific values caused participants to exhibit more behaviours consistent with the reinforced values, it is expected that an increase in the importance of ST values will predict participant's environmental behavioural intentions, while experimentally manipulating an increase in the importance of SE values will not predict participants' environmental behavioural intentions. These predictions are also based on findings from the meta-analytical summary described in Study 1 showing that ST values are the main values related to environmental engagement.

The two experiments are planned in a serial fashion where Experiment 2 will incorporate several improvements based on the results of Experiment 1 (e.g., two distinct samples and methodological procedures). The main reason for this approach is to tease out different procedures and replicate effects. The section that follows will present a detailed description of both experiments. 


\section{Experiment $1^{7}$}

Based on the literature summarised above, it can be argued that since the VSC technique is based on norm activation, then people who strongly identify with the relevant reference group are more prone to change values. Therefore, the core question is: what is the mechanism underlying the effect of the intervention? To test this question, Experiment 1 used VUW students as the reference group for the experimental manipulations. A student identity measure was also included and explored in this study.

Furthermore, in Experiment 1, a sample of university students from Victoria University of Wellington was used (and assessed in a laboratory setting) to examine if the VSC technique would have an effect on environmental behavioural intentions. As previously mentioned, Experiment 1 targeted three goals: 1) investigate if values change through the VSC technique, 2) investigate the impact of student identity on the mechanism of value change, and 3) test if value change predicts environmental intentions. Furthermore, it is reasonable to assume that student identity is a salient identity for the sample of university students assessed in Experiment 1. It was expected that values in Experiment 1 would change from pre-test to post-test. Furthermore, it was expected that prioritizing ST values would increase the importance of other ST values while at the same time decreasing the importance of SE values. It was also expected that prioritizing SE values would increase the importance of other SE values, while simultaneously decreasing the importance of ST values. Finally, no changes in value priorities would be observed for the control condition.

Regarding the impact of student identity on the mechanism of value change, it was expected that people who strongly identify with the reference group (therefore showing strong student identity) would be more willing to change values and conform to the reference group's values for both experimental conditions. On the other hand, it was expected that people who do not strongly identify with the reference group (therefore showing weak student identity) would be less willing to change values and, consequently, less willing to conform to the values prioritised by the reference group for both experimental conditions. It was also expected that no

\footnotetext{
${ }^{7}$ Some of the findings of this study were presented at an international conference. See Diniz, Milfont, McClure, and Fischer (2012).
} 
change would be observed for the control group. Regarding testing if value change predicts environmental intentions, it was expected that change in values would positively predict change environmental behavioural intentions.

\section{Method}

\section{Pre-test}

\section{Participants}

The participants in the pre-test were 571 undergraduate students enrolled in a first year psychology course at Victoria University of Wellington. The majority of the participants were female ( $n=361 ; 63 \%)$, and the remaining $(n=210 ; 37 \%)$ were male. The average age of participants was 19 years $(S D=3.79)$, ranging from 17 to 50 years.

\section{Materials and design}

The pre-test questionnaire included three critical measures: a list of values, a list of ecological behavioural intentions, and a group identification measure. These outcome measures are described below and presented in full in Appendices C, D, E, F, G, H, and I. Demographic questions were also included and participants provided information about age and sex, for example.

Values: The values measure presented an initial list of 16 values (Values 1) from the Schwartz Value Survey (Schwartz, 1992), with four values serving each of four higher order motivational clusters of values (i.e., ST, SE, openness to change, and conservation values). This research focused only on two higher order values dimensions that have been shown to be related to environmental issues: ST (loyalty, equality, helpfulness, and a world of peace) and SE values (ambition, social power, social recognition, and success). Each value was presented with a definition in parentheses (e.g., equality was defined as "equal opportunity to all"). Participants were asked to rank the values on the basis of their importance as guiding principles in their lives, such that the most important value was ranked 1 and the least important value was ranked 16. The first set of ST and SE values showed acceptable psychometric properties (polychoric ordinal alphas of .82 and .79 , respectively).

Ecological behavioural intentions. The dependent measure for this experiment was the 
General Ecological Behaviour (GEB) scale. The original version of the GEB scale is a composite of 50 performances proposed by Kaiser and Wilson (2004). Experiment 1 used a modified version of the GEB scale composed of items written as 'intention' items, describing ecological actions that the participants intend to engage in or not, for example, "reuse your shopping bag" or "be a member of a carpool". The items were answered on a 6-point Likert scale ranging from 0 (not at all willing) to 5 (extremely willing). Before running these experiments, a pilot study ${ }^{8}$ was conducted with a sample of 206 participants examining the reliability of the measure. A total of $62 \%$ female and $38 \%$ male composed the sample with mean age of 19 years old $(S D=3.47)$. This modified version of the GEB scale showed acceptable psychometric properties (i.e., Cronbach's alpha of .94). The measure was split into two scales based on the behavioural difficulty of the items (Kaiser et al., 2010), and both versions showed similar acceptable psychometric parameters (Cronbach's alphas for GEB-1 and GEB-2 were .89 and .87, respectively; the versions were also highly correlated, $r=.73$ ). GEB-1 was used in the pre-test and GEB-2 was used in the post-test of the present experiment. I included a distinct set of items in the pre-test and in the post-test to avoid transparency issues (i.e., participants being exposed to the same items twice and realizing the actual goal of the study).

Identity. A group identification measure was also included to tease out the effects of identity on the VSC technique. The identification measure used in this experiment was the Collective Self-Esteem scale (Luhtanen \& Crocker, 1992). This scale measures three aspects of identity: private (assesses how individuals privately evaluate their social group), public (assesses how individuals believe others evaluate their social group) and importance to identity (assesses the role of group memberships in the self-concept). The scale is composed of 12 items, with four items for each subscale. The items were adapted to express identification with Victoria University of Wellington (VUW) students. An example of an item for the private subscale is "In general, I am glad to be part of VUW"; an example for the public subscale is "Overall, VUW students are considered good by others"; and an example of item for the importance to identity subscale is "Belonging to VUW is an important reflection of who I am". People were instructed to answer the items on the basis of how they feel about their reference group by using a Likert

\footnotetext{
${ }^{8}$ Some of the findings of this study were presented at an international conference. See Diniz, Milfont, and McClure (2012).
} 
scale ranging from 1 (strongly disagree) to 7 (strongly agree). For this study an average score was computed for all the 12 items in the scale, and the scale showed acceptable psychometric properties (i.e., Cronbach's alpha of .84).

\section{Procedure}

This first phase of the experiment (pre-test) was completed during a mass testing of all first year psychology students. The participants were asked to sign up for the mass testing in exchange for course credit. They answered a set of measures assessing a varied number of topics in Psychology, including the three critical measures of interest for this study. The pre-test lasted one hour, with participants answering all questionnaires online in a group setting of a maximum of 16 people. This research (consisting of Experiments 1 and 2) was approved by the School of Psychology Human Ethics Committee under delegated authority of the Human Ethics Committee of the Victoria University of Wellington (Approved on 11 October 2011; Reference number: RM018524).

The present experiment was designed to overcome some potential limitations in Maio et al.'s (2009) design. Because Maio et al.'s participants completed pre- and post-measures in a single assessment, a limitation of their study is that participants might have become aware of the desired manipulation (i.e., the issue of experimental transparency). Experiment 1 in the present thesis was designed to overcome this limitation by having the pre- and post-measurement at least one week apart. The post-measurement is described below.

\section{Post-test}

\section{Participants}

A total of 189 university students who took part in the pre-test participated in the posttest. The majority of the participants were female $(n=105,56 \%)$, and the remaining were male $(n=84,44 \%)$. The average age of participants was 20 years $(S D=3.43)$, ranging from 17 to 47 years. According to a power analysis and previous research (Cohen, 1988), a minimum of 30 participants per cell was required. The present experiment had 64, 65 and 60 participants for each of the two experimental manipulations (ST and SE values conditions) and control conditions, respectively, in a 2 x 3 design with a total of six cells. 


\section{Material and design}

Three versions of the questionnaire were created representing the two experimental manipulations (ST and SE values conditions), and a control condition. In the post-test, participants answered another set of 16 different value items (Version 2) from the Schwartz Value Survey that tapped into the same four higher order domains of values as the pre-test. Again this research focused only on two higher order values dimensions (i.e., ST and SE values). This list of values was different from the list of values used in the pre-test measure. Participants were told that this set of values had not been used with students before (i.e., ST values: forgiveness, honesty, social justice, and broad-mindedness; SE values: authority, capability, influence, and wealth). They were asked again to rank the importance of these values as guiding principles in their own lives after the experimental manipulation (see below), such that the most important value was ranked 1 and the least important was ranked 16 (as in the pre-test). The second set of ST and SE values showed acceptable psychometric properties in the post-test (polychoric ordinal alpha of .81 and .83, respectively).

Additionally, the post-test questionnaire also included the second list of ecological behavioural intentions (GEB-2), and three filler questionnaires assessing well-being (the World Health Organization-Five Well-Being Index; WHO-5, 1998), mood (Positive and Negative Affect Schedule; PANAS; Watson, Clark, \& Tellegen, 1988), and self-esteem (Rosenberg SelfEsteem Scale; Rosenberg, 1965). The filler questionnaires were used to avoid transparency of the main goals of the research. Demographic questions were also included. A more detailed description of each phase of the experiment is described below. All measures used in this experiment are reported in Appendices C, D, E, F, G, H, and I.

\section{Procedure}

The post-test involved the experimental manipulation and the post-survey. This second session was performed in a laboratory with individuals who participated in the first session (pretest) and expressed willingness to participate in this second session (post-test). The post-test occurred approximately one week after the pre-test, with participants signing up for the experiment through an online enrollment system called Experimentrix. Participants were initially 
classified into groups with higher scores on ST values or higher scores on SE values according to their value rankings in the pre-test. This was to guarantee that the post-test sessions had a balanced number of participants who prioritised ST values and SE values in the pre-test. Participants were then randomly assigned to a session at the laboratory and placed in one of the two experimental conditions or in a control condition. On the day of the experiment, participants signed consent forms before completing the survey. They were then tested in a group setting with a maximum group size of six. At any one time, two experimental groups were run simultaneously. After all post-test sessions were completed at the end of the University term, an email was sent to participants debriefing them about the aims of the study.

\section{Experimental manipulation}

During the post-test, participants were randomly assigned to one of the two experimental conditions (ST and SE values condition) or to a control condition. The experimental and control conditions are detailed below.

Experimental conditions: There were two experimental conditions, one manipulated ST values, while the other condition manipulated SE values. In each condition, participants were shown the (fictitious) average value rankings of VUW students for the same values that were provided in the pre-test measure of values. After being presented with the fictitious value rankings, participants were first asked to read these rankings and their own rankings from the pre-test. They were then asked to identify the most highly ranked values in the reference group's ranking, and to write the names of the values in a predetermined column. Participants completed the same task for their own value ranking, and they were asked to identify the similarities and differences between their value rankings and the reference group's rankings.

Finally, participants were asked to read an explanation about the first preferred values of the reference group and about the characteristics of people who ranked those values as their most preferred values. The explanation was based on the descriptions of Schwartz's (1992) values theory. For the present study, an example of an explanation in the ST and SE values condition was adapted from Maio et al. (2009, p. 704) and is presented below:

\section{$\underline{\text { Self-transcendence }}$}

The average of the students' value ranking shows that the most important values to students at 
Victoria University of Wellington are loyalty, equality, helpfulness, and a world at peace. Past research demonstrated that people who believe in these values always emphasise universal human requirements, and are very interested in understanding, appreciating, tolerating, and protecting the welfare of all close others and people in other settings. Therefore, based on the average of students' rankings, we can conclude that they have shown their concern for the welfare of all human beings, even those whose way of life differs from theirs.

\section{$\underline{\text { Self-enhancement }}$}

The average of the students' value ranking shows that the most important values to students at Victoria University of Wellington are ambition, social power, social recognition, and successfulness. Past research demonstrated that people who believe in these values always emphasise achievement and personal success through demonstrating competence according to social standards and attainment of social status and prestige. Therefore, based on the average of students' rankings, we can conclude that they have shown their concern for active demonstration of competence in concrete interaction and attainment of a dominant position within a social system.

To guarantee that participants would read the explanation provided, the fictitious value rankings and the explanation were printed in colour-graded paper and handed out separately. To reinforce this aspect of the manipulation, they were also asked to write their own explanation of why students emphasised the given values.

Control condition: Participants in the control condition were asked to complete an unrelated measure. It included a list of "the desserts Victoria University students prefer the most". Participants were asked to rank a list of dessert items in terms of their preferences. They received a fictitious ranking of VUW students, and were asked to compare their rankings with the fictitious ranking. They also then received an explanation about VUW students' preferred desserts. The explanation was written in a format similar to that used in the experimental conditions. The text used in the control condition is presented below:

The average of the students' evaluations of what would be the best options for dessert showed lemon tart, macadamia caramel, blueberry muffin and apple crumble as the four favourites. Past research demonstrated that people who decide on flavours of food base their choices on personal preferences and dietary requirements. Therefore, based on the average of students' rankings, we can conclude that they have shown their preferences while taking into account their favourite desserts in their country. 


\section{Post-survey}

In the final task, participants received a booklet with six published measures. These measures included two critical measures: 1) a list of values (Version 2), and 2) the General Ecological Behaviour Scale (GEB-2); and the four filler questionnaires described in previous sections.

Additionally, at the end of the survey, questions about the participants' thoughts regarding the experiment were included. These questions had the aim of identifying if participants had any suspicion about the experimental hypotheses, more specifically if the research was examining value change and environmental behavioural intentions. If the participant identified that the goal of the study was to examine value change and environmental issues, they would be removed from analyses. However, no participant identified the real goal of the study, therefore all participants who took part in the study were included in the final data analysis.

\section{Analyses}

To examine the effects of the experimental manipulation on changes in value rankings from pre-test to post-test, a 2 (time: pre-test and post-test) x 2 (type of value: ST and SE values) x 3 (condition: ST condition, SE condition and control condition) mixed model ANOVA was conducted with repeated measures for the first two factors. The rank scores on the values measure were reversed so higher numbers would then indicate higher scores, and so indicating higher priority. To test the effects of identity on changes in values from pre-test to post-test, a 2 (time: pre-test and post-test) x 3 (condition: ST condition, SE condition and control condition) $\mathrm{x}$ 2 (identity: high and low student identity) mixed model ANOVA was conducted with repeated measures for the first factor. Prior to this analysis, the sample was divided into two groups formed by separating those who scored higher (above the median score of 4) and those who scored lower (below the median score of 4) on the identity measure. Finally, linear regressions were conducted to examine the effects of the value change on environmental behavioural intentions. 


\section{Results}

\section{Reliability and Descriptive Indexes}

Table 4.1 below shows the alpha coefficients, means, standard deviations and number of items for the three critical measures of interest for this study (Versions 1 and 2 of the attitudinal version of the General Ecological Behaviour scale, Lists 1 and 2 of the ST and SE values, and the student identity scale) as well as the three filler measures (Rosenberg's self-esteem scale, PANAS scale, and WHO-5). In general, the internal reliabilities were acceptable for all measures assessed (alphas above .78).

Table 4.1

Reliability and Descriptive Indexes of Measures Used for Experiment $1(n=189)$

\begin{tabular}{lccccc}
\hline & & $\alpha$ & $M$ & $S D$ & Number of items \\
\hline Environmental behavioural & GEB-1 & $.91^{\prime}$ & 2.56 & 0.73 & 14 \\
intentions & GEB-2 & $.86^{\prime}$ & 2.45 & 0.69 & 14 \\
Self-transcendence values & V1 & $.82^{*}$ & 10.63 & 2.30 & 4 \\
Self-enhancement values & V2 & $.81^{*}$ & 10.81 & 1.96 & 4 \\
& V1 & $.79^{*}$ & 7.96 & 2.19 & 4 \\
Student identity scale & V2 & $.83^{*}$ & 7.12 & 2.14 & 4 \\
Rosenberg's self-esteem scale & & $.84^{\prime}$ & 4.90 & 0.80 & 12 \\
PANAS scale & & $.89^{\prime}$ & 2.00 & 0.49 & 10 \\
& Positive affect & $.81^{\prime}$ & 3.37 & 0.60 & 10 \\
WHO-5 & Negative affect & $.87^{\prime}$ & 2.17 & 0.73 & 10 \\
\hline Polychoric ordina & & $.78^{\prime}$ & 2.94 & 0.82 & 5 \\
\hline
\end{tabular}

*Polychoric ordinal alphas (Gadermann, Guhn, \& Zumbo, 2012)

'Cronbach's alphas

\section{Time, Values and Condition}

The results of a mixed-factor ANOVA showed a significant main effect for time, Wilk's $\lambda=.93, F(1,183)=13.28, p<.001, \eta_{p}{ }^{2}=.07$. Values scores for the pre-test were significantly higher $(M=9.31, S D=0.08)$ than scores for the post-test $(M=8.98, S D=0.07)$. This supports 
the hypothesis that values would change from pre-test to post-test. A significant main effect was also found for type of value, Wilk's $\lambda=.51, F(1,183)=174.72, p<.001, \eta_{p}{ }^{2}=.49$. Participants scored higher on ST values $(M=10.76, S D=0.13)$ than they did on SE values $(M=7.54, S D=$ 0.14 ), indicating that participants prioritised values of universalism and benevolence over values of achievement and power.

The results of the mixed-factor ANOVA also showed a significant interaction between time and type of values, Wilk's $\lambda=.91, F(1,183)=16.95, p<.001, \eta_{p}{ }^{2}=.08$. These findings are reported in Figure 4.3 and showed that, as expected, scores on ST values significantly increased from pre-test $(M=10.04, S D=0.17)$ to post-test $(M=11.47, S D=0.14), p<0.05$, while scores on SE values significantly decreased from pre-test $(M=7.98, S D=0.17)$ to post-test $(M=7.09$, $S D=0.15), p<.001$. This indicates a greater change of ST values comparatively to SE values. The interaction between type of values and type of condition was marginally significant $(p<.10)$ while the interaction between time and type of condition was not significant $(p>.05)$. 


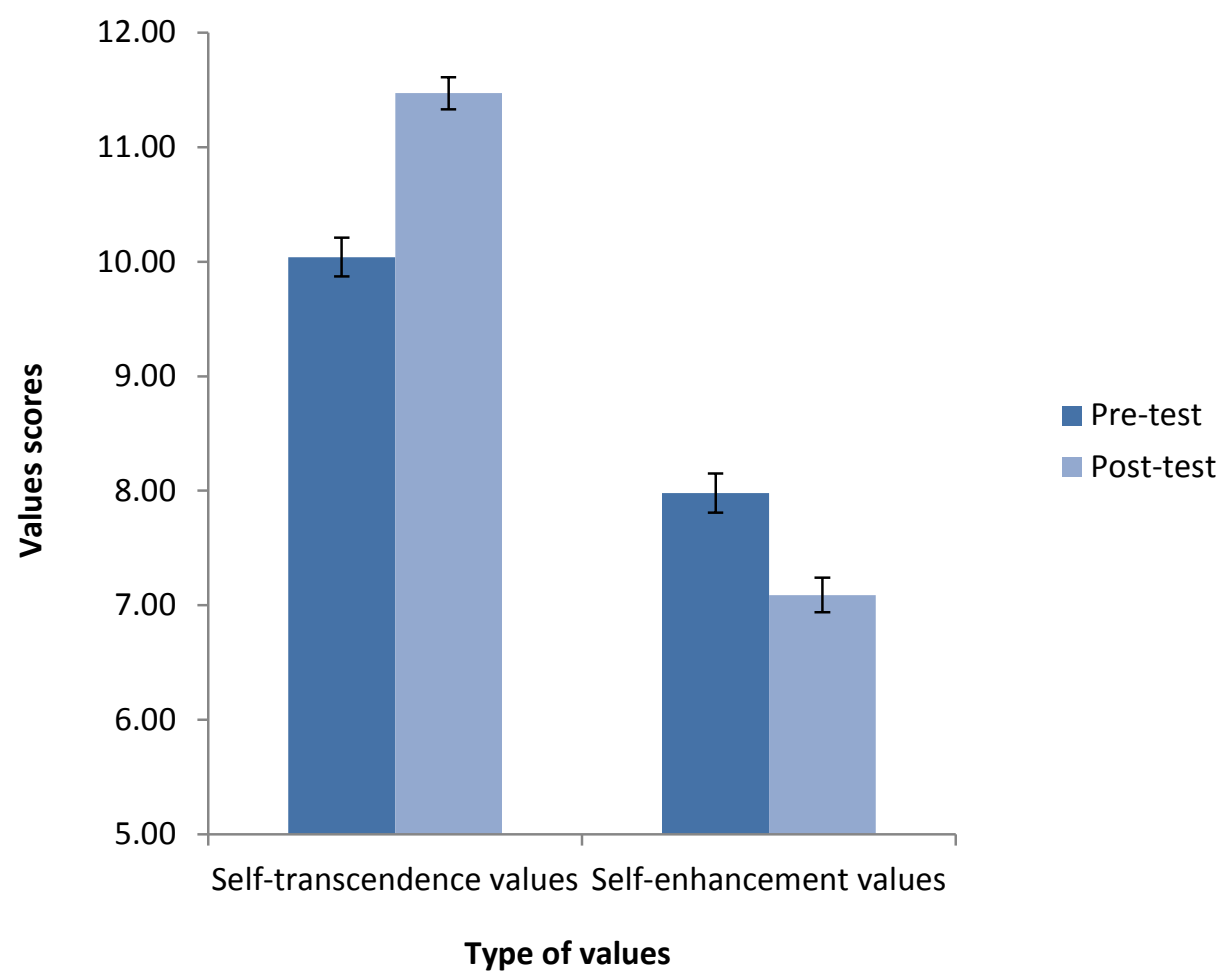

Figure 4.3. Values scores according to time and type of values.

Note: Error bars represent one standard error.

Importantly, a significant three-way interaction was found between time, type of value, and condition, Wilk's $\lambda=.93, F(1,183)=6.87, p<.001, \eta_{p}{ }^{2}=.07$, indicating that scores in each condition differed significantly from pre-test to post-test and between ST and SE values. The results of this three-way interaction are presented in Figure 4.4 below. As expected, in the ST condition, scores on ST values significantly increased from pre-test $(M=10.13, S D=0.29)$ to post-test $(M=11.13, S D=0.24), p<.001$, whereas scores on SE values significantly decreased from pre-test $(M=8.14, S D=0.30)$ to post-test $(M=6.74, S D=0.27), p<.001$. This finding supports the hypotheses that prioritizing ST values would increase the importance of other ST values but decrease the importance of SE values. In the SE condition, scores on ST values decreased from pre-test $(M=10.50, S D=0.29)$ to post-test $(M=10.16, S D=0.23)$, but this was not significant, $p>.05$, and consequently did not support the hypothesis that prioritizing SE 
values would decrease the importance of ST values. Unexpectedly, also in the SE condition, scores on SE values decreased from pre-test $(M=7.89, S D=.30)$ to post-test $(M=7.61, S D=$ 0.26), but this was not significant, $p>.05$, and the hypothesis that prioritizing SE values would increase the importance of other SE values was not supported. In the control condition, scores on ST values remained relatively unchanged from pre-test $(M=11.30, S D=0.30)$ to post-test $(M=$ 11.33, $S D=0.31), p>.05$, but scores on $S E$ values decreased from pre-test $(M=7.93, S D=$ $0.31)$ to post-test $(M=6.91, S D=0.27), p<.001$. Based on these results, the hypothesis that no change on value priorities would be found for the control condition was only partially supported. 


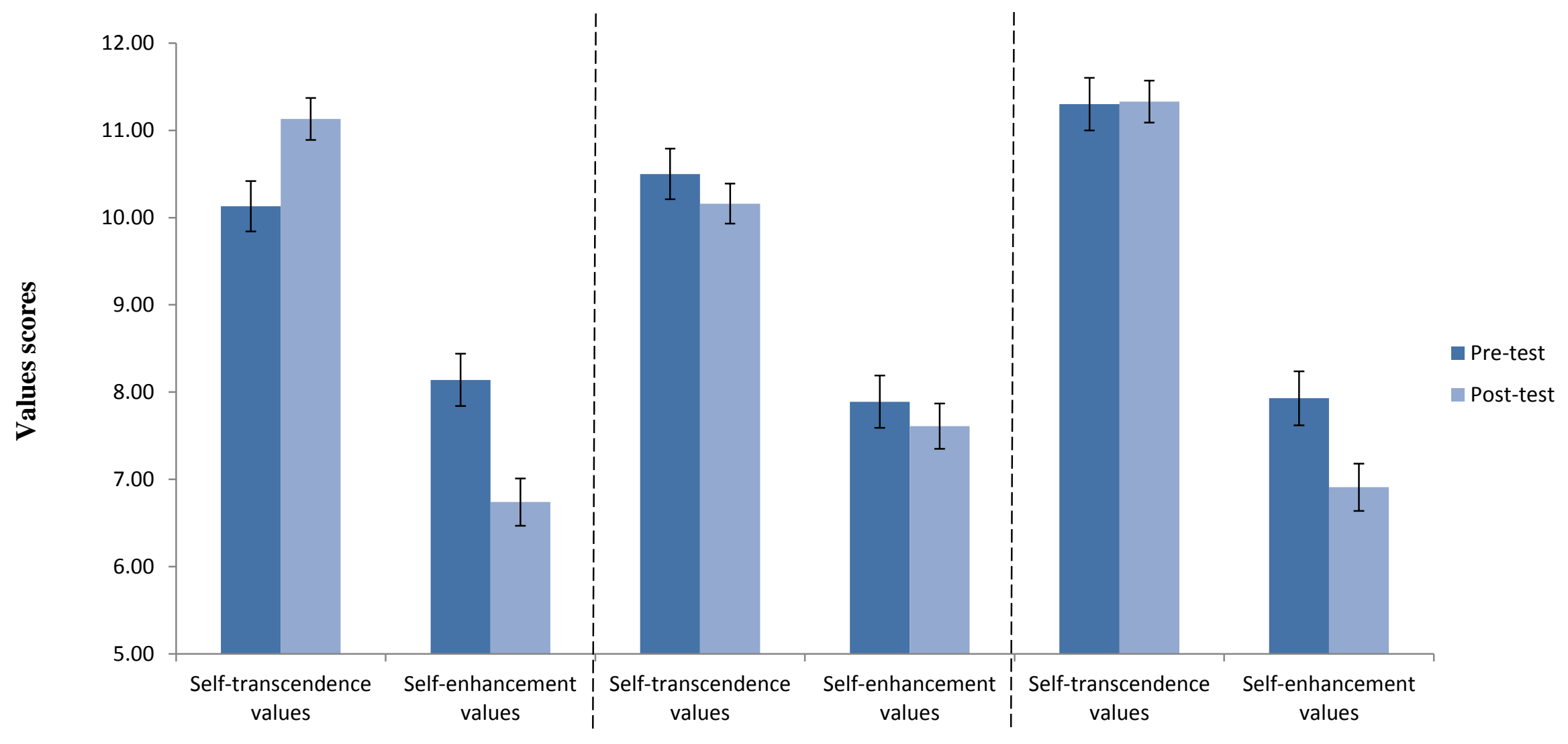

Self-transcendence condition

Self-enhancement condition

Control condition

Figure 4.4. Values scores according to type of condition (self-transcendence, self-enhancement and control conditions), type of values (self-transcendence and self-enhancement values) and time (pre-test and post-test).

Note: Error bars represent one standard error. 


\section{Time, Condition and Student Identity}

Having shown that ST values changed in the expected direction after the manipulation, additional analyses were conducted to examine the influence of identity on the experimental design. Specifically, SE values scores were deducted from ST values scores to test for the value of ST over SE (with higher scores indicating greater difference between ST and SE values). Furthermore, the calculation of a difference score isolates ST values by removing the influence of SE values. A mixed model ANOVA was conducted with time (pre-test vs. post-test) as a repeated measure variable, and condition (ST, SE and control conditions) and identity (low and high student identity) as between subjects variables.

The results showed neither a significant main effect of time nor a significant interaction between time and identity scores $(p>.05)$. However, a significant interaction between time and condition was found (see Figure 4.5 below), Wilk's $\lambda=.93, F(1,176)=6.11, p<.001, \eta_{p}{ }^{2}=.06$. As expected, ST minus SE values scores in the ST condition significantly increased from pre-test $(M=10.10, S D=2.16)$ to post-test $(M=11.05, S D=2.28), p<.001$. Whereas ST minus SE values scores in the SE condition decreased from pre-test $(M=10.59, S D=2.14)$ to post-test $(M$ $=10.24, S D=1.93), p>.05$, but this was not significant, contrary to what was expected. Finally, as expected, ST minus SE values scores in the control condition did not exhibit much change from pre-test $(M=11.31, S D=2.43)$ to post-test $(M=11.26, S D=1.99), p>.05$. These results indicated that ST minus SE values are more likely to change if ST values are reinforced instead of SE values. 


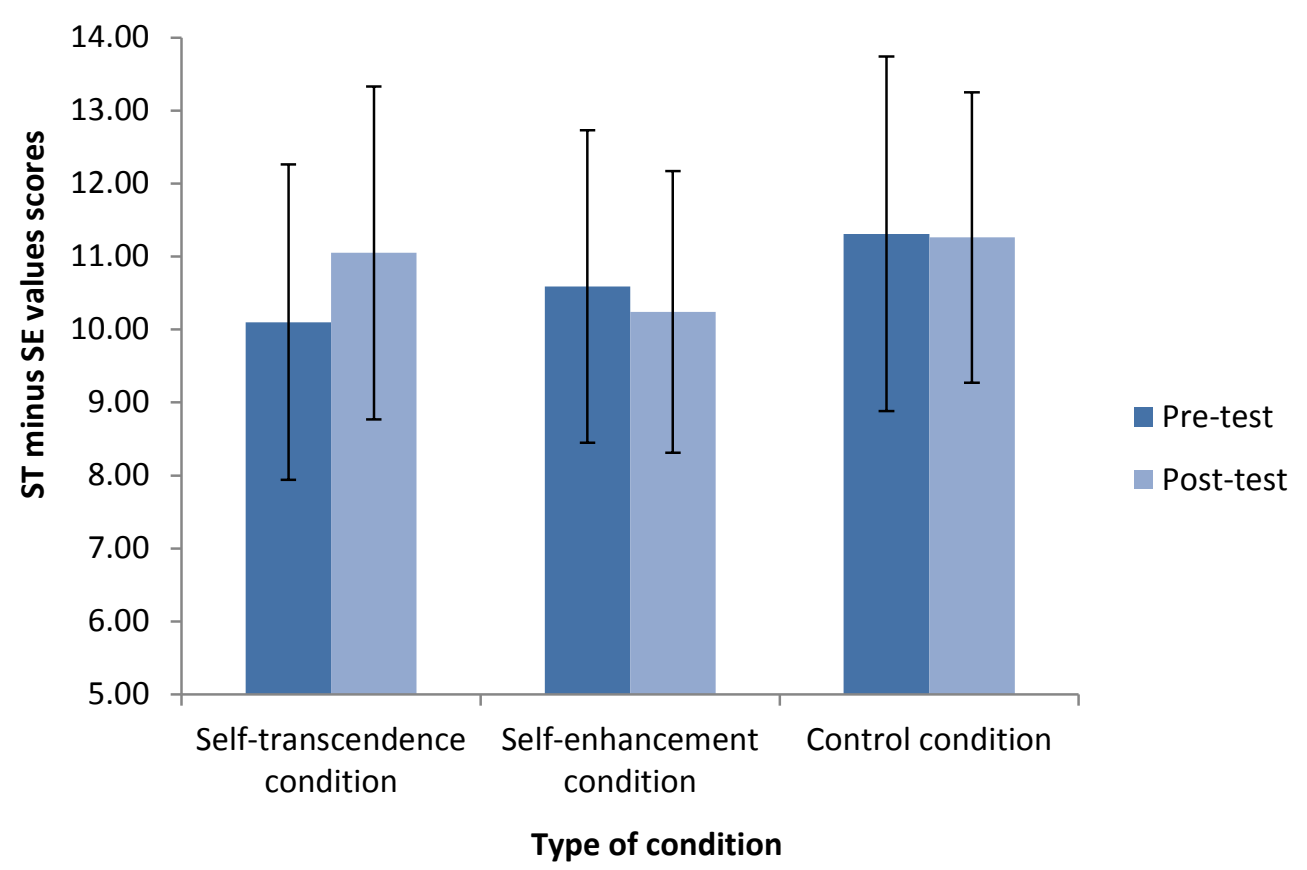

Figure 4.5. Self-transcendence minus self-enhancement values scores according to time (pre-test and post-test) and type of condition (selftranscendence, self-enhancement and control conditions).

Note: Error bars represent one standard error.

The expected three-way interaction between time, type of condition and student identity was not significant (Wilk's $\lambda=.98, F(1,176)=1.30, p>.05, \eta_{p}{ }^{2}=.02$ ). However, although nonsignificant, results show a trend in both groups of low and high identity scores (see Figure 4.6 below). For example, Figure 4.6 shows that, for the low identity scores group (A), as expected, the ST minus SE values scores increased from pre-test to post-test in the ST condition (pre-test $M=9.97, S D=2.48$ and post-test $M=10.84, S D=1.76, p<.01)$. Contrary to what was expected, the ST and SE values scores also increased from pre-test to post-test in the SE condition (pre-test $M=10.23, S D=1.83$ and post-test $M=11.27, S D=1.69, p<.01$ ). Nevertheless, as expected, no significant change was found for the control condition (pre-test $M$ $=11.23, S D=2.74$ and post-test $M=11.45, S D=1.98, p>.05$ ). Figure 4.6 also shows that for the high identity scores group (B) the ST minus SE values scores increased as expected from pretest $(M=10.23, S D=1.83)$ to post-test $(M=11.27, S D=1.69), p<.05$ for the ST condition 
while decreased from pre-test $(M=11.02, S D=2.01)$ to post-test $(M=10.17, S D=2.16), p<$ .01 for the SE condition (which is expected with a sample of people who scored higher in ST values). Unexpectedly, for the control condition ST minus SE values decreased from pre-test ( $M$ $=11.32, S D=1.94)$ to post-test $(M=10.99, S D=2.02), p<.01$. Overall this trend supports the prediction that, for participants with high student identity, ST values would increase in the ST condition, whereas they would decrease in the SE condition.

To test if the control group was affecting the results of the experimental conditions, the same mixed-model ANOVA was conducted by excluding the control group from the analysis. The results were similar to what was found when the control group was in the analysis showing a significant interaction between time and condition $(p<.05)$ but no significant three-way interaction between time, type of condition and identity $(p>.05)$. Also as expected, the results for the high identity scores group followed the same pattern as the one found in the previous analyses: ST minus SE values scores significantly increased from pre-test $(M=10.14, S D=$ 2.81 ) to post-test $(M=11.39, S D=1.55), p<.01$ for the $\mathrm{ST}$ condition, while the $\mathrm{ST}$ minus $\mathrm{SE}$ values scores decreased from pre-test $(M=10.86, S D=2.02)$ to post-test $(M=9.31, S D=2.21)$ for the SE condition, $p<.05$. This indicates that people who highly identified with the reference group, changed their ST minus SE values scores accordingly to what was prioritised by the reference group (i.e., ST or SE values) in both experimental conditions. 


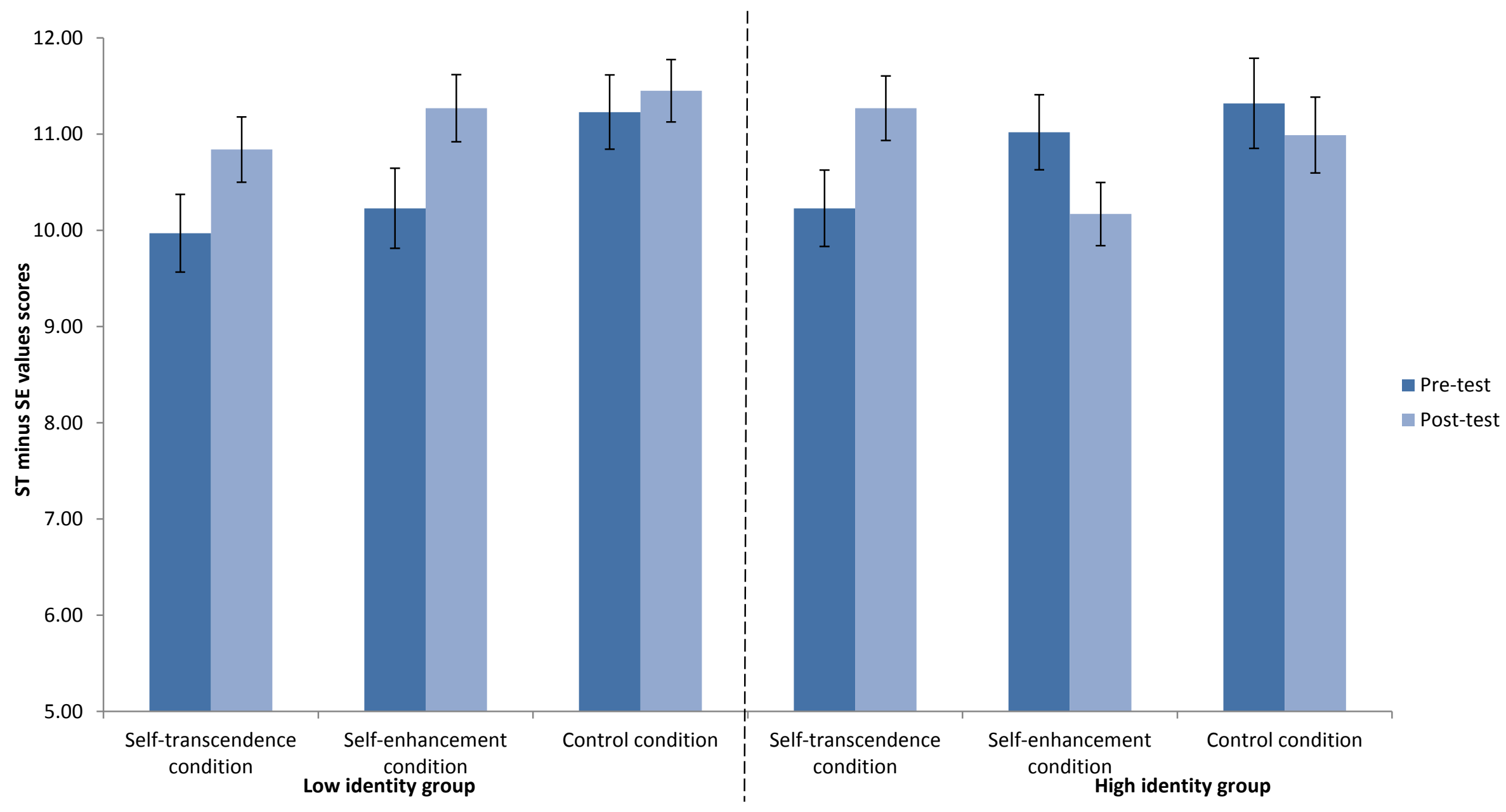

Figure 4.6. Self-transcendence minus self-enhancement values scores according to time (pre-test and post-test) and type of condition (self-transcendence, self-enhancement and control conditions) in low (A) and high identity score (B) groups.

Note: Error bars represent one standard error. 


\section{Change on Values and Environmental Behavioural Intentions}

A paired samples t-test was conducted to examine if the environmental behavioural intentions scores changed from pre-test to post-test. The results showed a small marginally significant change in environmental behavioural intentions from pre-test to post-test, $t(181)=$ $1.85, p<.10, d=.11)$, with behavioural intentions surprisingly decreasing from pre-test $(M=$ $2.52, S D=0.75)$ to post-test $(M=2.44, S D=0.69)$.

Change scores were created based on the algebraic difference between post-test and pretest scores on the values measure [(ST minus SE values scores at post-test) - (ST minus SE values scores at pre-test)] and on the environmental behavioural intentions measure [(GEB-2 GEB-1)]. Regression analyses were performed with the environmental behavioural intentions change score regressed onto the independent variable of value change. Results from the regression analysis showed a non-significant prediction of value change for environmental behavioural intentions change from pre-test to post-test $(p>.05)$. A regression analysis was then performed with the environmental behavioural intentions scores at post-test regressed onto the independent variable of value change. Results from this analysis showed that the regression was significant $(F(1,191)=13.31, p<.05)$ and the independent variable explained $7 \%$ of the variance in environmental behavioural intentions at post-test $\left(R^{2}=.07\right)$. Examination of the standardised coefficients revealed that, as predicted, change on ST minus SE values was a positive predictor of environmental behavioural intentions of participants after the experimental manipulation $(\beta=.26, p<.001)$.

\section{Discussion}

This experiment examined whether values could be modified, whether identity plays a role in value change and whether this induced value change influenced environmental behavioural intentions. The experiment used the value self-confrontation (VSC) technique which has been shown to be a valid tool to induce value change (Maio et al., 2009; Schwartz \& InbarSaban, 1988). Results from Experiment 1 showed that participants scored higher on ST values at baseline, indicating that they are more concerned about promoting the welfare of others rather than their own. Additionally, it was found a significant interaction between time and type of values (Figure 1) indicating that ST values increased from pre-test to post-test while SE values decreased from pre-test to post-test. Although condition is not considered in this interaction, and 
therefore it is not possible to assess whether the manipulation had an effect, this result indicates that there was a greater change on ST values compared to SE values, which is in line with findings reported by Maio et al. (2009).

Most importantly, the expected three-way interaction between time, type of values and type of condition was significant (Figure 2), indicating that values changed from pre-test to posttest depending on the experimental condition and on the type of values assessed. This also indicates that the pattern of value change for ST and SE values across experimental conditions differed. Looking at the single comparisons in the graph (Figure 2) we can note that some results confirmed what was expected while others were contrary to our hypothesis. Specifically, for the ST condition, ST and SE values changed in the expected directions, indicating that when ST values are prioritized by the reference group, a characteristic of the ST condition, they exert influence on the values priorities of the participants by increasing their ST values and decreasing their SE values. This follows the theoretical principal of the VSC technique (Rokeach, 1973; Maio et al., 2009) which argues that when people are confronted with certain types of values they tend to conform to the values prioritised by the reference group. People do this by increasing the priority they put onto those values (in this case ST values) and reducing the priority they put onto values of the opposing dimension (in this case SE values). In Experiment 1 , this only occurred in the ST condition, when ST values were made salient.

On the other hand, in the SE condition, no significant change was observed between pretest and post-test for either ST or SE values. This means that manipulating SE values did not have the expected influence on subsequently measured ST and SE values as observed when manipulating ST values. It is important to note that Maio et al. (2009) found the expected effect of manipulating both ST and SE values on subsequent measured values also for student samples. But perhaps social desirability might have played a role in the present sample. The SE values are usually considered socially undesirable (e.g., Sedikides, Gaertner, \& Toguchi, 2003) and so the present students might not have been inclined to increase their endorsement of SE values after the experimental manipulation. In conclusion, using SE values as a priming value in the VSC technique might not work as well as other values such as ST values. This is an issue to be further examined in future studies.

In turn, when time, condition and student identity were analysed the results showed that although there was no significant main effect of time, nor interaction between time and identity, 
a significant interaction between time and condition was found (Figure 3). The results from this significant two way interaction indicated that ST minus SE values scores increased in the ST condition while there was no significant change on values from pre-test to post-test in the SE condition. Also as expected no significant ST minus SE values scores change was observed in the control condition. This indicates that ST minus SE values are more prone to change when people are exposed to an ST condition than to an SE condition. This follows what was found in the previous analysis showing that ST condition was more able to elicit value change than the SE condition.

Furthermore, the expected three-way interaction between time, type of condition and student identity was not significant (Figure 4). One reason might be that overall, the participants do not identify with university students. They are in their first year at university and may identify with another reference group instead, for example, New Zealanders. Although the three-way interaction was not significant indicating that the pattern of results are similar for the two groups, there are still significant differences within the conditions allowing comparisons between them (Figure 4). For the low identity group, ST minus SE values scores increased from pre-test to post-test as expected for the ST condition but unexpectedly also increased for the SE condition. One possible reason for this is that for the low identity group the VSC technique may not work as well as it does for the high identity group. Students who do not identify with the reference group of university students may not really care about the values preferences of fellow students. Using the same explanation, students who do strongly identify as being a student should be more prone to the effects of social comparison with the student reference group. Indeed, participants in the high identity group did exhibit positive change in their ST minus SE values scores for both experimental conditions. In general, this experiment demonstrated that ST values can be more easily influenced than SE values and that this is especially true when participants strongly identify with the reference group.

Furthermore, although the induced ST values change did not predict change on environmental behavioural intentions from pre-test to post-test, the ST values change significantly predicted environmental behavioural intentions at post-test. Experiment 2 was designed to replicate these findings with a general population sample (and not students) to investigate the moderating role of identity when the reference group is a national group. Experiment 2 addresses this research question. 


\section{Experiment $2^{9}$}

Experiment 2 went beyond the previous experiment with the goal of testing if the results could be replicated outside of the laboratory. Also, and most importantly, Experiment 2 aimed to further test the influence of identity. As discussed previously, the VSC technique is based on norm activation and people who identify more with the reference group are expected to show more change on values. Therefore, the core question again is: what drives the effect of the intervention? To test this question, a national identity measure (instead of a student identity measure) was included and explored in this study. The reference group used was 'New Zealanders' instead of 'VUW students'.

Furthermore, in Experiment 2, a survey design with a sample of participants from the general population of New Zealanders (instead of undergraduate students) was used to examine if a different method of data collection and different population would produce similar results as Experiment 1, and whether the VSC technique moderated by national identity (instead of student identity) would have an effect on environmental behavioural intentions.

As with Experiment 1, it was expected that values in Experiment 2 would also change from pre-test to post-test. More specifically, it was expected that prioritizing ST values would increase the importance of other ST values while at the same time decreasing the importance of SE values. In comparison, highlighting SE values would increase the importance of other SE values, while simultaneously decreasing the importance of ST values. No changes in value priorities were expected for the control condition. It was also expected that people who strongly identify with the reference group (therefore showing high national identity) would be more willing to change values and conform to the reference group values for both experimental conditions. On the other hand, it was expected that people who do not strongly identify with the reference group (therefore showing low national identity) would be less willing to change values and, consequently, less willing to conform to the values prioritised by the reference group for both experimental conditions. No change was expected for the control group. It was also

\footnotetext{
${ }^{9}$ Some of the findings of this study were presented at international conferences. See Diniz, Milfont, Fischer, and McClure (2013b, 2013c).
} 
expected that change in values would positively predict change in environmental intentions.

\section{Method}

\section{Participants}

A total of 138 members of the general population took part in the study, but only 115 were considered for the analysis. Twenty-three participants were excluded for the following reasons: a) they did not complete all the questions in the survey; b) they suspected that the survey was looking at value change; or c) they were not born in New Zealand. The final sample had an average age of 35 years $(S D=14.61)$ ranging from 18 to 76 years. Regarding gender, the majority of the 115 participants were males $(n=60,52.2 \%)$, while the remainder were females $(n=55,47.8 \%)$. There were 42, 36, and 37 participants for each, ST, SE and control conditions, respectively. Also, a balanced number of females and males were included in each condition.

\section{Procedure}

Participants were approached in public places (i.e., a shopping mall, a public library, and cafes) and asked to participate in the study. They were given an information sheet instructing them about the overall goals of the study, their consent to participate, and their freedom to withdraw at any time. Participants were then given a single questionnaire containing pre-test, manipulation, and post-test to complete in as much time as they needed. Participants took an average of thirty minutes to complete the questionnaire. After completing the survey, they were given a debriefing sheet informing them of the manipulation and the purpose of the study, and received a thank-you note and a chocolate bar as a reward for their participation.

\section{Material and design}

Experiment 2 is a questionnaire-based experiment where, in contrast to Experiment 1, all phases of the experiment were carried out in a single time-point (i.e., pre-test, experimental manipulation, and post-test). The pre-test questionnaire included the first list of values (Version 1), GEB-1, and the group identification measure (identification with New Zealanders instead of VUW students). Three versions of the questionnaire were created referring to two experimental manipulations (ST and SE values conditions), and a control condition. Finally, the post-test questionnaire included the second list of values (Version 2), the GEB-2, and two filler measures 
assessing mood (Positive and Negative Affect Schedule; PANAS; Watson et al., 1988) and selfesteem (Rosenberg Self-Esteem Scale; Rosenberg, 1965). Demographic questions were also included. All measures used in this experiment are reported in Appendices J, K, L, M, and N and E and the reliability indexes (Cronbach's alpha and polychoric ordinal alpha) of all measures used in Experiment 2 are presented in Table 4.2.

\section{Analyses}

The statistical analyses conducted for Experiment 2 were similar to those employed in Experiment 1. To examine the effects of the manipulation on changes in value rankings from pre-test to post-test, a 2 (time: pre-test and post-test) x 2 (type of values: ST and SE values) x 3 (condition: ST condition, SE condition and control condition) mixed model ANOVA was conducted with repeated measures for the first two factors. Again only two higher order dimensions of values (ST and SE values dimensions) were considered in the analyses and the ranked values were reversed so higher scores indicated higher priorities.

Because New Zealand identity did not interact with other factors, this variable was not included in the initial analysis. However, to test the effect of national identity on the VSC technique, this variable was included as a factor in a later analysis. In particular, to test the effects of identity on changes in values from pre-test to post-test, a 2 (time: pre-test and post-test) x 3 (condition: ST condition, SE condition and control condition) x 2 (identity: high and low national identity) mixed model ANOVA was conducted with repeated measures for the first factor. As in Experiment 1, prior to this analysis the sample was divided into two groups formed by those who scored higher (above the median score of 4) and those who scored lower (below the median score of 4) on the identity measure. Finally, linear regressions were conducted to examine the effects of value change on environmental behavioural intentions. 


\section{Results}

\section{Reliability and Descriptive Indexes}

Table 4.2 below shows the alpha coefficients, means, standard deviations and number of items for the three critical measures of interest for this study. This includes Versions 1 and 2 of the attitudinal version of the General Ecological Behaviour scale, Lists 1 and 2 of the ST and SE values, the national identity scale and two filler measures (Rosenberg's self-esteem scale and PANAS scale). As seen in Table 4.2, the internal reliabilities were high for all measures assessed (alphas above .80).

Table 4.2

Reliability and Descriptive Indexes of Measures Used for Experiment 2

\begin{tabular}{lcccccc}
\hline & & $\alpha$ & $M$ & $S D$ & Number of items \\
\hline Environmental behavioural & GEB-1 & .86 & 2.74 & 0.67 & 14 \\
intentions & GEB-2 & $.84^{\prime}$ & 2.60 & 0.66 & 14 \\
& V1 & $.80^{*}$ & 10.46 & 2.24 & 4 \\
Self-transcendence values & V2 & $.81^{*}$ & 10.81 & 2.26 & 4 \\
& V1 & $.82 *$ & 7.01 & 2.26 & 4 \\
Self-enhancement values & V2 & $.80^{*}$ & 6.71 & 1.95 & 4 \\
& & $.86^{\prime}$ & 4.12 & 0.35 & 12 \\
National identity scale & & $.88^{\prime}$ & 1.90 & 0.50 & 10 \\
Rosenberg's self-esteem scale & & $.899^{\prime}$ & 3.63 & 0.73 & 10 \\
PANAS scale & Positive affect & Negative affect & $.88^{\prime}$ & 2.18 & 0.80 & 10 \\
\hline
\end{tabular}

*Polychoric ordinal alphas (Gadermann et al., 2012).

'Cronbach's alphas

Time, Values and Conditions

The results of the mixed-factor ANOVA showed a significant main effect of type of values, Wilk's $\lambda=.42, F(1,110)=154.03, p<.001, \eta_{p}{ }^{2}=.58$, such that overall, participants scored higher on ST values $(M=10.62, S D=0.16)$ than they did on SE values $(M=6.90, S D=$ 
0.17), as was found in Experiment 1. The main effect of time was not significant $(p>.05)$, suggesting that values did not change from pre-test to post-test (but see below).

A significant interaction was observed between type of values and conditions, Wilk's $\lambda=$ $.91, F(2,110)=5.33, p<.01, \eta_{p}{ }^{2}=.09$. As expected, participants scored higher on ST values in the ST condition $(M=11.21, S D=0.27)$, than they did in the SE condition $(M=10.14, S D=$ $0.29, p<.01)$. However, contrary to what was expected, there was not a significant difference between participants scores on ST values in the ST condition compared to the control condition $(M=10.52, S D=0.29, p>.05)$. As expected, participants scored higher on SE values in the SE condition $(M=7.59, S D=0.29)$ than they did in the ST condition $(M=6.28, S E=0.27, p<$ .001). However, there was not a significant difference between participants scores on SE values in the SE condition compared to the control condition $(M=6.82, S D=0.29, p>.05)$. These results are presented in Figure 4.7 below.

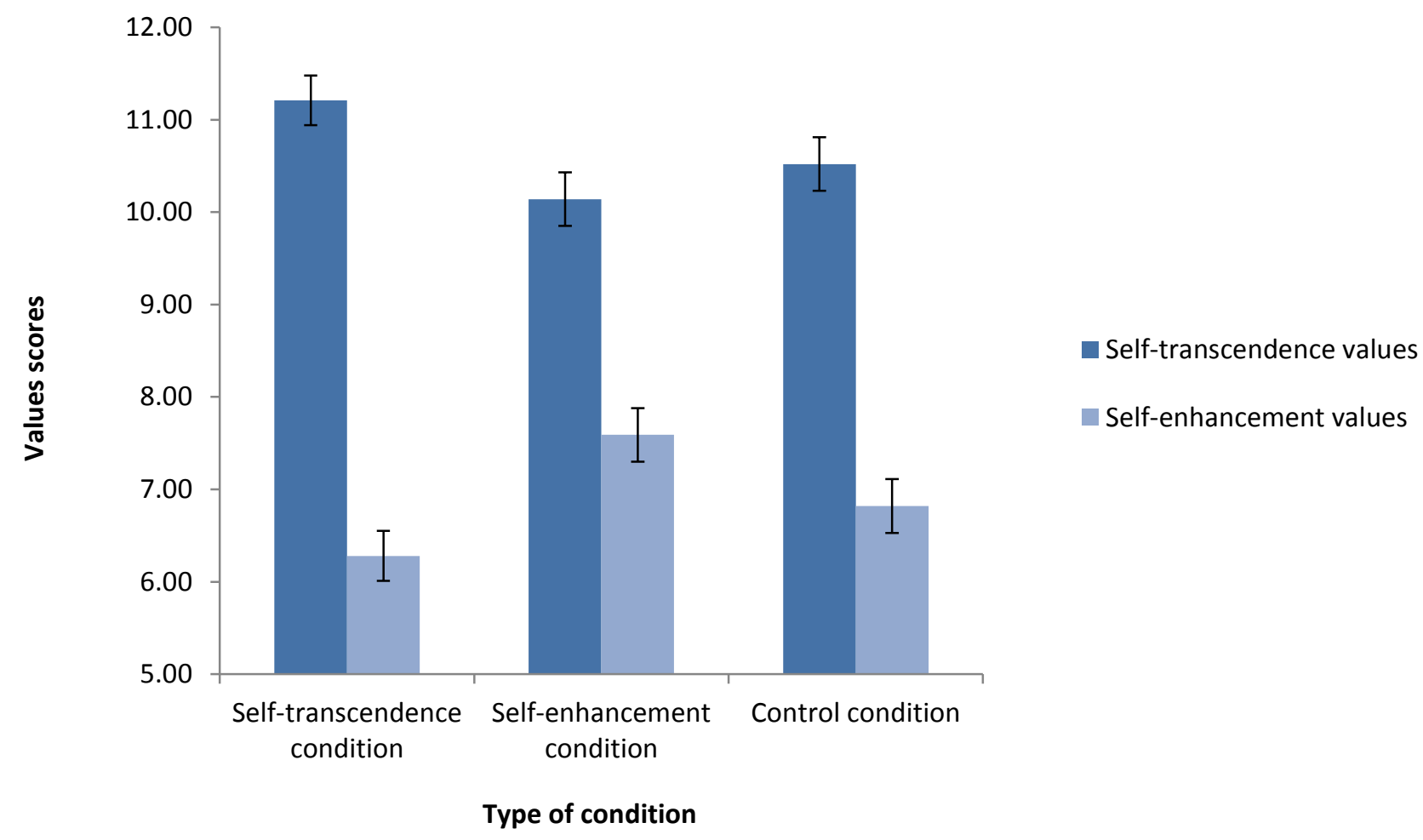

Figure 4.7. Values scores according to type of values (self-transcendence and self-enhancement values) and type of condition (self-transcendence, self-enhancement, and control conditions).

Note: Error bars represent one standard error. 
In addition, as expected, there was a significant interaction between time and the type of values (see Figure 4.8 below), Wilk's $\lambda=.95, F(1,110)=5.49, p<.05, \eta_{p}{ }^{2}=.05$. However, the single comparisons indicated that ST values did not change much over time (pre-test $M=11.06$, $S E=2.25$; and post-test $M=11.35, S E=1.60), p>.05$, similarly to $S E$ values that did not show greater change from pre-test $(M=7.73, S D=2.55)$ to post-test $(M=7.43, S E=2.37, p>.05)$. Additionally, an interaction between time and type of condition was not observed $(p>.05)$.

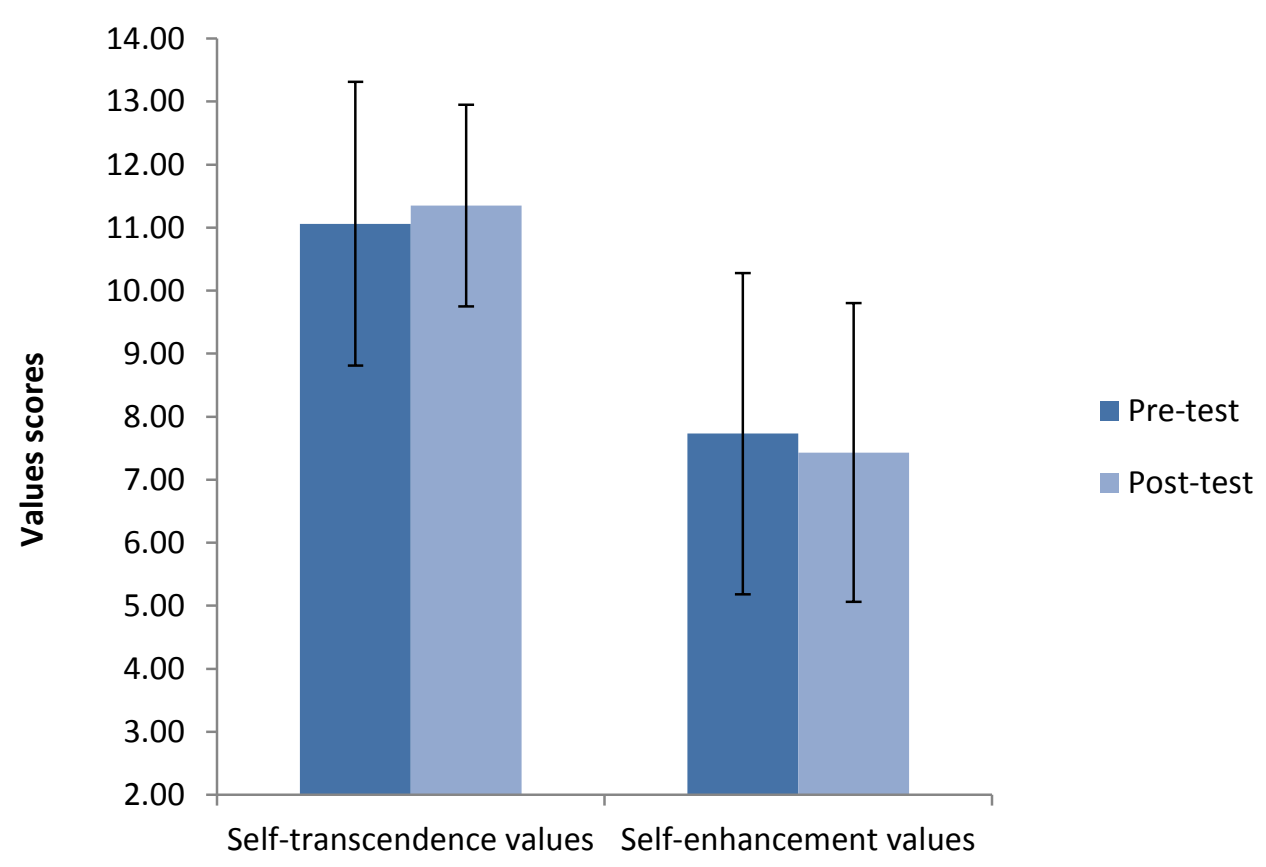

Type of values

Figure 4.8. Values scores according to time (pre-test and post-test) and type of values (self-transcendence and self-enhancement values).

Note: Error bars represent one standard error.

Also, and most importantly, the expected three-way interaction amongst time, type of values and condition was not significant $(p>.05)$, see Figure 4.9. The single comparisons also did not reveal any significant results $(p>.05)$. Moreover, the results do not confirm the hypotheses that prioritizing ST values would increase the importance of other ST values while decreasing the importance of SE values, whereas prioritizing SE values would increase the 
importance of other SE values while decreasing the importance of ST values. This result differed from Experiment 1. 


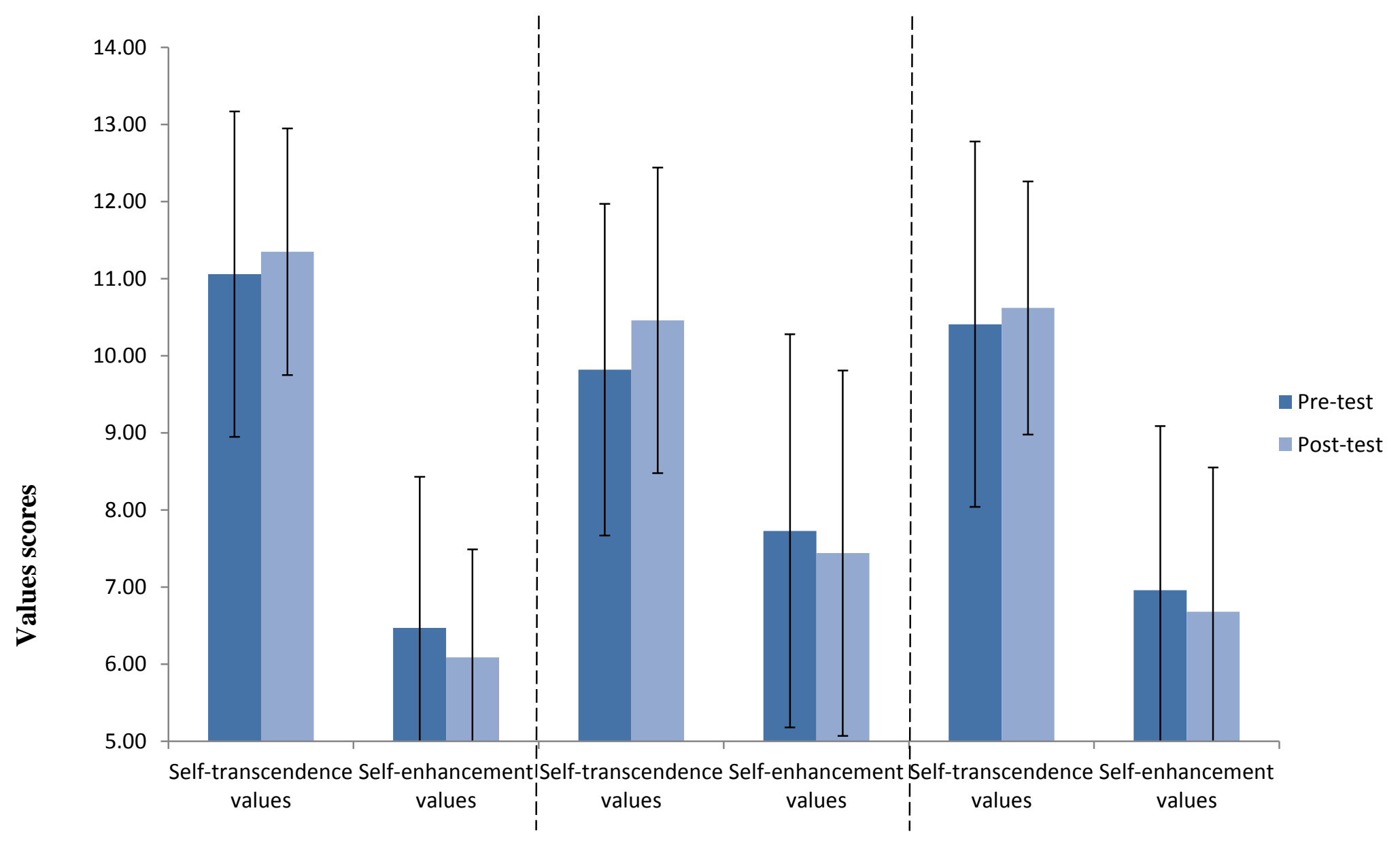

Self-transcendence condition

Self-enhancement condition

Control condition

Figure 4.9. Values scores according to type of condition (self-transcendence, self-enhancement and control conditions), type of values (self-transcendence and self-enhancement values) and time (pre-test and post-test).

Note: Error bars represent one standard error. 
Although the results of the mixed-factor ANOVA showed a non-significant main effect of time, as reported above, this non-significant result might have been influenced by the number of factors considered in the previous analysis. Therefore, to further test if values changed from pre-test to post-test, a one-way ANOVA was performed on the algebraic difference between the pre-test (ST values minus SE values on the pre-test) and post-test rankings (ST values minus SE values on the post-test). As expected, the results showed a significant change in values from pretest to post-test, Wilk's $\lambda=.95, F(1,110)=7.14, p<.01, \eta_{p}{ }^{2}=.05$. Also, as expected, the values difference score was greater in the post-test $(M=4.26, S D=3.19)$ than it was in the pre-test $(M$ $=3.52, S D=3.84)$. These results provide some support for the hypothesis that participants would change values priorities from pre-test to post-test.

\section{Time, Condition and National Identity}

To test how identity would affect the VSC technique in Experiment 2, further analyses were conducted only with ST values (ST minus SE values scores), since it was evident in Experiment 1 that, compared to SE values, the ST dimension was susceptible to greater change. Similar to Experiment 1, a mixed model ANOVA was conducted with time (pre-test vs. posttest) as a repeated measure variable, and type of condition (ST, SE and control conditions) and identity (high and low identity) as between subjects variables.

The results showed a main effect of time (Wilk's $\lambda=.95, F(1,106)=5.92, p<.05, \eta_{\mathrm{p}}^{2}=$ $.05)$, indicating that scores for the post-test $(M=10.80, S D=1.78)$ were significantly higher than scores for the pre-test $(M=10.43, S D=2.24)$, confirming the hypothesis that values changed from pre-test to post-test. The interaction between time and type of condition as well as the interaction between time and identity were not significant $(p>.05)$. However, the results did show a significant interaction amongst time, condition, and New Zealand identity (Wilk's $\lambda=$ $\left..90, F(2,106)=5.74, p<.01, \eta_{\mathrm{p}}{ }^{2}=.10\right)$. These results are reported in Figure 4.10 below. 


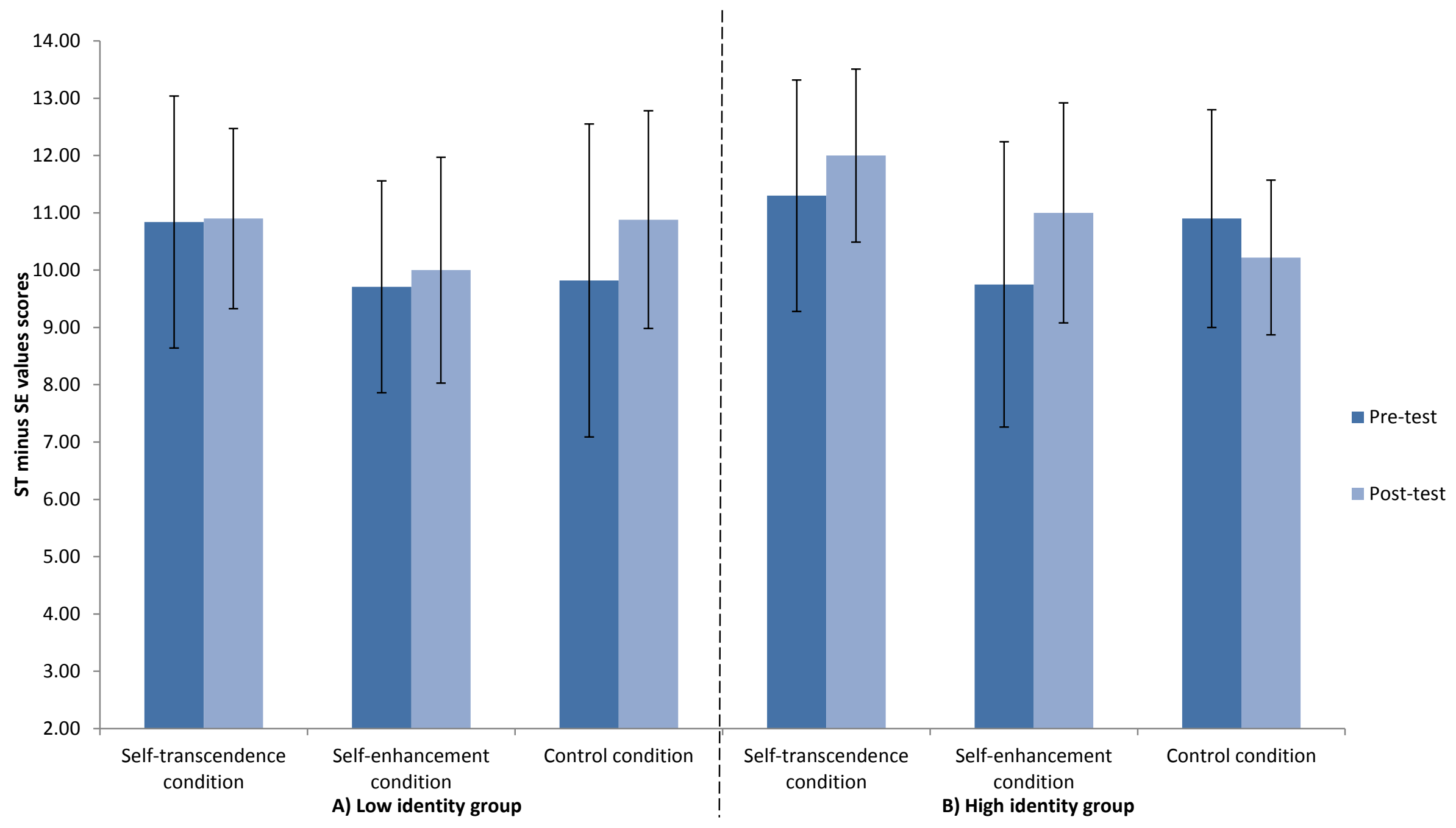

Figure 4.10. Self-transcendence minus self-enhancement values scores according to time (pre-test and post-test) and type of condition (self-transcendence, self-enhancement and control conditions) in low (A) and high (B) national identity groups.

Note: Error bars represent one standard error. 
As can be seen in Figure 4.10, the low identity scores group did not change in ST minus SE values scores from pre-test $(M=10.84, S D=2.20)$ to post-test $(M=10.90, S D=$ $1.57, p>.05)$, in the ST condition. Similarly, in the SE condition, there was not great change in ST minus SE values scores from pre-test $(M=9.71, S D=1.85)$ to post-test $(M=10.00, S D$ $=1.97, p>.05)$. These results confirm the hypothesis that a smaller or not significant value change would be expected for the low identity group. Finally, the control group showed an unexpected significant increase on ST minus SE values scores from pre-test $(M=9.82, S D=$ $2.73)$ to post-test $(M=10.88, S D=1.90, p<.05)$, not confirming the hypothesis that no change would be observed for the control group.

However, for the high identity scores group (see Figure 4.10), as expected, there was an increase in ST minus SE values scores in the ST condition (pre-test: $M=11.31, S D=2.02$; post-test: $M=12.00, S D=1.51, p<.05)$, but unexpectedly, they also increased in the SE condition (pre-test: $M=9.75, S D=2.49$; post-test: $M=11.00, S D=1.92, p<.01$ ). As expected, there was not a great change in ST minus SE values scores in the control condition from pre-test to post-test (pre-test: $M=10.9, S D=1.90$; post-test: $M=10.22, S D=1.35, p$ > .05). These results partially confirmed the hypotheses, as ST values scores increased in the ST condition, but unexpectedly, they also increased in the SE condition for the high identity group. Specifically, the effect is more pronounced and followed the predicted directions, for participants in the ST condition who strongly identify as being a New Zealander.

With the aim of testing if this difference between pre-test and post-test was significant for each condition in the higher identity scores group for ST minus SE values scores, t-tests were conducted for each of the two experimental and control conditions. As expected, the ttests showed that there was a significant difference between pre-test and post-test on ST minus SE values scores for the ST condition, $t(18)=24.39, p<.001, d=0.39$. Specifically, in the ST condition participants scored higher at the post-test $(M=12.00, S D=1.51)$ than at the pre-test $(M=11.31, S D=2.02)$. Similarly, a significant difference was found for the SE condition, $t(14)=15.17, p<.001, d=0.56$, whereas participants scored higher at the post-test $(M=11.00, S D=1.92)$ than at the pre-test $(M=9.75, S D=2.48)$. However, in the control condition, a significant difference was found in the opposite direction, $t(18)=25.06, p<$ $.001, d=0.41$. Specifically, participants scored slightly higher at pre-test $(M=10.90, S D=$ 1.90) compared to the post-test $(M=10.22, S D=1.35)$.

An ANOVA was then conducted to examine the differences between ST, SE and 
control conditions from pre-test to post-test (simple contrast). As expected, the results showed a significant difference (contrast estimate $=1.03 ; S D=0.56 ; p<.05 ; d=0.67$ ) between the ST $(M=11.32, S D=2.02)$ and control condition $(M=10, S D=1.90)$ at the pretest, and at the post-test [(contrast estimate $=1.66 ; S D=0.52 ; p<.01 ; d=1.16$; ST condition $(M=11.89, S D=1.51)$; control condition $(M=10.22, S D=1.35)]$. However, there was no difference between SE and control conditions at either the pre-test or post-test (contrast estimate $=-0.19, S D=0.59, p>.05$; contrast estimate $=0.78, S D=0.55, p>.05$, respectively).

\section{Change in Values and Environmental Behavioural Intentions}

Similar to Experiment 1, a paired samples t-test was conducted to examine if the environmental behavioural intentions scores changed from pre-test to post-test. The results showed a small significant change in environmental behavioural intentions from pre-test to post-test, $t(96)=3.44, p<.001, d=0.22$ ), with environmental behavioural intentions surprisingly decreasing from pre-test $(M=2.73, S D=0.67)$ to post-test $(M=2.58, S D=$ $0.66)$.

Also similar to Experiment 1, change scores were created based on the algebraic difference between post-test and pre-test scores on the values measure [(ST minus SE values scores at post-test) - (ST minus SE values scores at pre-test)] and on the environmental behavioural intentions measure [(GEB-2 - GEB-1)]. Regression analyses were then performed with the environmental behavioural intentions change score regressed onto the independent variable of value change. Results from the regression analysis showed that changes on ST minus SE values scores did not significantly predict the changes on environmental behavioural intentions from pre-test to post-test $(p>.05)$. Similar to Experiment 1, regression analyses were then performed with the environmental behavioural intentions scores at post-test regressed onto the independent variable of value change. Results from this analysis showed that the regression was significant $(F(1,102)=10.08, p<.01)$ and the independent variable of value change explained $8 \%$ of the variance in environmental behavioural intentions at the post-test $\left(R^{2}=0.08\right)$. Examination of the standardised coefficients revealed that, as predicted, change in ST minus SE values was a positive predictor of participants environmental behavioural intentions after the experimental manipulation $(\beta=.30, p<.01)$. 


\section{Discussion}

Experiment 2 did not confirm the hypothesis that values are susceptible to change using the VSC technique. Although a main effect of type of values was found indicating that, similar to Experiment 1, participants scored higher on ST values and consequently tend to be more concerned about others rather than themselves; values did not change significantly from pre-test to post-test and a significant three-way interaction was not observed. In general, the non-significant three-way interaction between time, type of values and condition indicated that there was no significant change in the pattern of results from pre-test to post-test. In addition, a closer look at the single comparisons also showed no significant results (see Figure 7). These results go against previous findings obtained by Maio et al. (2009) and those reported in Experiment 1.

However, when time, condition and national identity were analysed the results showed that values changed, increasing from pre-test to post-test. More importantly, a significant three-way interaction was found indicating that the pattern of results is different amongst the different groups. The single comparisons show that for the low identity group there was not a change on ST minus SE values scores from pre-test to post-test for the two experimental conditions. This indicates that, as expected, participants who do not identify with the reference group also do not change their values by conforming to the value priorities of the reference group. A closer look at the high identity group shows that participants that highly identify with New Zealanders tend to increase their ST minus SE values scores when primed with ST values, as expected. Whereas, contrary to expectations, when participants who highly identified with being a New Zealander were primed with SE values in the SE condition, they showed an increase in their ST minus SE values scores rather than a decrease. This unexpected effect could be explained by social desirability since SE values emphasise materialism, power, achievement and participants might be more reluctant to endorse such values. It can also be argued that SE values are less aligned with the values promoted in New Zealand than ST values. Therefore, participants may not have been convinced by the manipulation in the SE condition for the New Zealand reference group. Furthermore, although an unexpected change was found for ST minus SE values scores from pre-test to post-test in the control conditions for the low and high identity groups, subsequent analysis showed that the ST condition differed from the control condition, while the SE condition did not differ from the control condition. In conclusion, these results indicate that the VSC technique is more effective for ST values than when priming SE values. 
Additionally, the present experiment aimed at testing if value change would be a good predictor of behavioural intentions. The results did not confirm the hypothesis that a change in specific values (especially ST values) would predict change in environmental behavioural intentions. Interestingly, and as observed in Experiment 1, the results showed that behavioural intentions decreased rather than increased from pre-test to post-test. Although several efforts were undertaken to produce two balanced versions of the GEB measure, it is possible that there were still differences between the environmental behavioural intentions assessed in the pre-test and the post-test that may be accounting for this contradictory result. Nevertheless, the induced changes in ST values predicted environmental behavioural intentions at the post-test, confirming the results observed in Experiment 1, and reinforcing a core finding of this thesis.

\section{General Discussion}

In general, the results from Experiments 1 and 2 show three main findings: (1) values can change, (2) the effect is moderated by student and national identity, and (3) change in ST values can predict environmental behavioural intentions. These results are discussed in more detail below.

First, the experimental findings provide support for the VSC technique. In particular, the main findings of Experiment 1 showed that the VSC technique works in a very specific and controlled setting with a specific sample of university students. Changes in values were observed from pre-test to post-test which supported the expected directions for the ST condition but not for the SE condition, where no changes were observed. This results indicated that ST values are more easily manipulated, or it is more effective to prime them compared to SE values. Additionally, it may be possible that social desirability may have masked potential changes on SE values. In Experiment 2, although results did not reveal a significant change in values across the experimental conditions like those observed in Experiment 1, there was still an indication (revealed by results from the one-way ANOVA) in Experiment 2 that values changed from pre-test to post-test. As emphasised in a previous section of this chapter, research by Rokeach (1973), Schwartz and Inbar-Saban (1988), and Maio et al. (2009) are a few examples of studies that have employed the VSC technique to change values. However, they did not have a specific focus on the effect of change in values on environmental engagement. The results from the two experiments in this chapter provide novel empirical evidence for the effectiveness of the VSC, and also show that there is a 
greater change in ST values relative to SE values.

Furthermore, despite the overall effect of the VSC technique in both samples, the effect was stronger for the student sample in Experiment 1 compared to the general population sample in Experiment 2. One explanation for these results is that students seemed to be more malleable with regard to values and environmental behavioural intentions than the general population. One possible reason for that is the different age range of the two samples. The general population sample was older (age ranging from 18 to 76 years old; $M=35$ ) than the university students sample (age ranging from 17 to 47 years old, with the majority aged between 18 and 22 years old; $M=20$ ). Although values structures can be found at younger ages (Doring et al., 2015), other studies (e.g., Achenreiner, 1997) have shown that values are still being formed in youth because they are more prone to change, while values in older aged participants are more stable. Other studies report age differences in resistance to peer influence (e.g., Steinberg \& Monahan, 2007), showing that susceptibility to peer pressure increases during adolescence and declines in older age groups. Although these findings suggest that age differences might have influenced some of the results, there is a consistent pattern across both experiments showing the moderating role of both student and national identities, as discussed below.

The second point addressed by Experiments 1 and 2 refers to the effect of student and national identity on value change. The experimental findings showed that the mechanism of value change works for those in the ST condition who highly identify as being a student, and for those who highly identify as a New Zealander. Although a significant three-way interaction with student identity was not observed in Experiment 1, an overall trend emerged. The experimental manipulation had a somewhat greater effect on participants with greater identification with the reference group of university students, evidenced by the participants' higher levels of ST values after the manipulation. It is possible that in the low identity group the VSC technique may not have worked as well as it does for the high identity group because students who do not identify with the reference group of university students may not care very much about the values preferences of other students. Using the same rationale, students who do strongly identify as being a VUW student are more prone to the effects of social comparison with the student reference group and, consequently, change their values accordingly. This was confirmed in Experiment 2 with a general population sample, with identification as a New Zealander playing an important role in value change. In this experiment, the three-way interaction was statistically significant indicating that New 
Zealand-born participants were more prone to change ST values from pre-test to post-test if they strongly identified with being a New Zealander (the reference group in the experimental manipulation). In other words, change in ST values due to the experimental manipulation was greater for participants with stronger identification with the national group and exposed to the ST condition.

However, when participants with high identification with the national group were exposed to the SE condition, their SE values scores did not increase as expected. This also differed from Experiment 1, where participants who highly identified with the reference group exhibited a change of values in the expected direction for both conditions. One possible explanation for this divergence is that for Experiment 2 participants were older than the participants in Experiment 1, and so have likely strongly internalized New Zealand values. Additionally, it can be argued that SE values are less aligned with the values promoted in New Zealand than ST values. Studies have shown that New Zealanders place importance on group goals (e.g., family, tribal group or some other collective set) goals over personal goals (e.g., Podsiadlowski \& Fox, 2011), suggesting that New Zealanders are more oriented by ST values compared to SE values (which in turn emphasise materialism, power, achievement, etc.). Because of that, it is possible that participants exposed to the SE condition may not have been convinced by the manipulation that emphasised SE values as the supposedly most relevant values of the New Zealand reference group. Overall, results from both experiments suggest that the effect of the SE manipulation on value change is not as consistent as the effect of the ST manipulation. As noted above, this could also be due to social desirability but needs to be explored in future research.

These findings underpin the argument that identity salience affects change in values, particularly ST values. It has been suggested that value change works through norm activation (see Maio et al., 2009), and the likelihood of promoting an effective change in values is underlined by social comparison. Furthermore, the literature suggests that place of attachment and identity are important factors that determine the relevance of the social group for an individual and how likely this person is to accept pressure from others (e.g., Chen et al., 2007; Chernoff \& Davison, 2005). In other words, people are more likely to change if they identify strongly with a reference group and previous research has shown that participants are more willing to change their behaviour and engage in socially expected behaviours when compared to a group norm (Chernoff \& Davison, 2005). The present experimental results support the role of identity (student identity and national identity) on the 
effectiveness of the VSC technique for value change. The findings also add important insight to the literature, as so far studies have focused on relating values and identity separately to explain environmental engagement (e.g., Fielding et al., 2008; Nigbur et al., 2010; Schultz et al., 2005). In sum, the present experimental studies provide empirical support for the role of identity (student identity and national identity) as a central mechanism for value change.

Finally, the experimental results show that changes on ST values predict environmental behavioural intentions measured after the experimental manipulation. To the author's knowledge, there are no studies that have focused on investigating the mechanisms of value change directly assessing environmental outcomes. The results of the two experiments described in the present thesis were consistent with the finding that changes in values predict environmental behavioural intentions. Furthermore, the meta-analytical results reported in Study 1 confirmed that ST values are the main predictors of environmental engagement. In turn, the present experimental results suggest that after experimentally inducing ST values, these values predict environmental behavioural intentions in the posttest. Put together, these results indicate that ST values can be enhanced and that these values can foster environmental engagement. Importantly, the present results go beyond previous studies (e.g., Milfont, Sibley, \& Duckitt, 2010; Schultz \& Zelezny, 1999; Stern et al., 1998) and Study 1 by showing that the link between ST values and environmental engagement can be fostered by inducing value change through identity.

Surprisingly, the results also indicated a significant decrease in environmental intentions from pre-test to post-test in both experiments. This could be a result of the set of items used. Although the items were equally selected in terms of their difficulty and both sets were correlated (see page 74 of this thesis), it is still possible that behaviours assessed with pre-test items were easier to perform compared to behaviours measured by the post-test items. Inspection of the items support this explanation. For example, the post-test items of "buy solar panels to produce energy" and "contribute financially to environmental organizations" are seemingly harder to perform compared to the pre-test items of "reuse your shopping bags" and "look into the pros and cons of having a private source of solar power". Additionally, it is possible that the items in the second version of the GEB (i.e., GEB-2) referred to actions that were not appealing to older participants in the sample from the general population. For example, some older participants in this experiment mentioned that installing solar panels in their house may not be desirable due to high financial investment and small return from taking this action. Future research could explore these explanations. 
Overall, it is important to note that Experiments 1 and 2 complement each other, although there were differences between them. One of the main differences is that Experiment 1 was performed with a sample of university students in a laboratory setting, whereas Experiment 2 was conducted with a sample of the general population in nonlaboratory settings. By providing an examination of the predicted effects across two distinct samples and methodological procedures, it is believed that a better understanding of the underlying mechanisms of value change was achieved. The experiments also made it possible to provide a comparison with the laboratory and non-laboratory results. Finally, both experiments included large number of participants per cell. Each cell of the experiments had more than thirty two participants. This was twice as many as the power analysis (Cohen, 1988) suggested me to include.

\section{Limitations}

The two experiments described in this study complement each other and set out to overcome limitations of previous studies using the same technique (such as those conducted by Maio et al., 2009), while testing out variations in procedures. However, some limitations need to be acknowledged. First of all, the two experiments do not strictly follow the same procedure. This is because of the origin of the samples - Experiment 1 includes university students while Experiment 2 includes the general population who did not come to the laboratory. Because of this aspect, the general population sample could not have been controlled in terms of its initial values scores. In addition, the pre-test and post-test were conducted at the same time point. Despite these limitations, the design of Experiment 2 provides access to participants from varied age groups and different backgrounds, and is not limited to a university setting. This enabled testing the effect of one's identification with being a New Zealander. This effect would potentially be possible to test in a student sample, but would probably not have shown a strong effect, as at this age students may still be forming their national identity (Sartor \& Youniss, 2002).

Another limitation is the ceiling effect of ST values, where the number of participants scoring high on ST values was higher than the number of participants scoring high on SE values at pre-test in both experiments. A possible explanation for this disparity is that most people do not want to be seen as self-centred. SE values are usually associated with being selfish, a characteristic that although common in individualistic cultures is often undesirable (Sedikides et al., 2003). Being selfish has negative implications for the individual, such as 
hostile impressions and social exclusion (Leary et al., 1997; Paulhus, 1998). Research has shown that independent of their collectivistic or individualistic orientation or background, people engage in socially desirable responses (see Lalwani, Shavitt, \& Johnson, 2006, for a review). Therefore, the higher scores on ST values might reflect participants' intention to not appear selfish, masking the true scores of participants on the values measure. In other words, social desirability may have acted as a confounding variable influencing the results.

As already discussed, different sets of values and GEB measures were included at two different times of these experiments (pre-test and post-test). It can be argued that different sets of the same measure can generate different results, and this is a possible limitation of the present study. However, particularly different sets of values were used as part of the VSC technique which requires the use of distinctive items for values in the experimental manipulation (see Maio et al., 2009). Furthermore, different sets of the GEB were used to prevent the participants from realizing the true goals of the experiments (the issue of transparency) and, as already discussed in previous sections of this thesis, the two sets of GEB items showed strong correlation with each other and good reliability indices, attesting that both sets of the same measure were reliable and equivalent.

Another potential limitation of Experiments 1 and 2 is the use of a median split to create two groups based on low and high identity scores. Although median split is a common practice to create dichotomous variables from participants' scores, there have been criticisms of this statistical procedure. For example, MacCallum, Zhang, Preacher, and Rucker (2002) stated that a median split can incur loss of power in the analysis and it treats scores just above or below the median as equal to the scores at the end of the scale. This limitation is acknowledged but a median split provided the most parsimonious approach for conducting the analysis with two distinct groups.

Another limitation of Experiments 1 and 2 is the absence of a manipulation check. Manipulation checks help to determine whether the manipulation met its intended effect as well as providing evidence for construct validity of the manipulation (e.g., Cozby, 2009; Clark, 2011). Although the lack of a manipulation check does not mean that we are unable to draw any conclusions regarding whether the experimental manipulation caused the variation in the dependent variable, it is recommended that future experiments include a manipulation check to further examine the validity of the VSC technique on value change.

Finally, although Experiment 1 used a specific strategy to avoid the transparency of the goals of the experiment (i.e., having pre- and post-test at two different time points), this 
was not achieved with Experiment 2. In Experiment 2 other strategies (i.e., the use of filler questionnaires) were implemented to hide the real goals of the study from the participants, and each phase of the experiment (pre-test, experimental manipulation and post-test) was conducted at one time point. This could have potentially contributed to making the values manipulation too transparent to the participants which consequently could have affected the results. However, at the end of the post-test survey the debriefing questions enquired participants about the goals of the study and participants were removed from analysis if they identified the real goal of the study. Therefore, transparency issues were unlikely to have influenced the results of Experiment 2.

\section{Practical Applications of the Results}

This set of experiments is one of the first endeavours to assess changes in values using an experimental approach while investigating the role of identity as a mechanism of this change, and the effect of this change on environmental behavioural intentions. The present research can inform campaigns that attempt to elicit environmentally friendly behavioural intentions. The findings show that the VSC technique can be efficiently employed to promote value change, and that identity plays an important role in this process. It is reinforced that identity should be considered as it helps to promote changes in the priority of values that, consequently, affects environmental engagement. Furthermore, the findings from this research may offer a more effective solution to current behaviour change programs that rely almost exclusively on environmental education, a technique that is typically ineffective, as pointed out by Schultz and Kaiser (2012).

The way that campaigns are designed should critically contemplate the impacts that values depicted in their messages have on people's intentions. Campaigns are often targeted to large audiences and it is important that they have the desired effect on the targeted environmental intentions (and consequent behaviours). Fostering the appropriate range of values by promoting ST values instead of SE values may facilitate the ability of ST values to guide intentions. It also can avoid any undesirable side effects, as research has shown that focusing on SE values can undercut the ability of ST values to guide environmental behaviour (Evans et al., 2012).

To conclude, this set of two experiments offered insights into the importance of identity as a variable underlying the mechanisms of value change and how value change explains environmental behavioural intentions. The next chapter will take this research one 
step further and examine how another type of identity and other moderator variables may influence the link between values and environmental behavioural intentions. 


\section{CHAPTER FIVE: STUDY 3}

\section{Testing the Moderator Effect of Individual Differences in the Relationship between Values and Environmental Behavioural Intentions}

Results from the meta-analysis on values and environmental engagement presented in Chapter 3 showed that values, specifically ST values, predicted environmental engagement. In Chapter 4, the results of the experiments showed that the centrality of values could be manipulated to promote value change. More interestingly, the findings also showed that student and national identity influenced the process of value change. Additionally, Experiments 1 and 2 showed that the change in ST values predicted environmental behavioural intentions. All together, the findings presented in Chapters 3 and 4 suggested that values are linked to environmental engagement and that student and national identity can influence this relationship. The present study will further investigate the effect that other individual variables may also have on the link between values and environmental behavioural intentions.

Past research and the results reported in previous chapters of this thesis have shown that values are motivational goals that can lead to more environmental engagement. As a result of this, individuals need to be motivated to act in environmentally responsible ways. Additionally, previous research suggests that, as well as student and national identity, values and pro-environmental engagement are shaped by a number of other variables (e.g., Gifford, 2008). Some of these variables include moral identity (Aquino \& Reed, 2002), self-control (Baumeister \& Exline, 1999), self-efficacy (Schwarzer \& Jerusalem, 1995), and consideration of future consequences (Strathman, Gleicher, Boninger, \& Edwards, 1994).

\section{Why Moral Identity, Self-control, Self-efficacy and CFC?}

In previous experiments, student and national identity significantly influenced value change, which in turn predicted environmental behavioural intentions. These findings suggested the importance of considering identity as an essential construct to understand the mechanism underlying value change, which in turn influences the values-environmental behavioural intentions link. The literature has suggested that moral identity is intrinsically related to values (Aquino \& Reed, 2002) as well as being a good predictor of pro- 
environmental behaviours (Hardy, Walker, Olsen, Woodbury, \& Hickman, 2014). Thus, it seems reasonable to suggest that moral identity may moderate the relationship between values and environmental outcomes. Moral identity is defined by Aquino and Reed (2002) as a "self-conception organised around a set of moral traits" (p. 1424). Furthermore, the authors characterised moral identity as rooted in a trait-based conceptualisation and linked to specific moral traits based on social-cognition-oriented definitions of the self; i.e., a distinct mental image of what a moral person is like with regard to what they think, feel, and do. People with a high moral identity are likely to show greater integrity or morality (Aquino \& Reed, 2002) and therefore there might be a stronger relationship between endorsing values that are related to environmental outcomes and acting according to them.

Besides moral identity, other moderators were included in this study. The main goal of these inclusions was to tease apart the effects of different possible moderators of the relationship between values and environmental behavioural intentions. These variables were chosen because of their empirical or theoretical relationship with values and their empirical prediction of environmental outcomes. For example, studies have reported that self-control is by definition intrinsically related to values, especially when self-control refers to the ability to control impulses, act morally, and support the pursuit of long-term goals. There is also evidence showing that self-control is a predictor of environmental behaviour (Bamberg \& Moser, 2007) and it is also closely related to self-efficacy.

In turn, self-efficacy refers to the belief that one is capable of performing certain behaviours (Bandura, 1986). Although the relationships between self-efficacy and values have not been widely explored in the literature, empirical results have shown that selfefficacy predicts environmental engagement (Milfont, 2012; Park \& Yang, 2012; Tabernero \& Hernandez, 2011).

Another variable that has been shown to be a predictor of environmental engagement is CFC, also defined as people's ability to think about the future and anticipate the consequences of their actions (Strathman et al., 1994). Although, there is no empirical evidence yet of its connection with values, this construct has been largely studied in relation to environmental engagement. Research shows that people's greater CFC predicts environmental attitudes and behaviours (Milfont et al., 2012).

Because of these reasons, it seems reasonable to suggest that values would only influence environmental engagement for individuals who: (a) believe their actions are 
morally important or see themselves as holding strong moral traits, (b) feel they have the strength to actively resist counterproductive habits, (c) consider themselves capable of performing a particular task, and (d) realise the long-term consequences of their actions. Thus moral identity, self-control, self-efficacy, and CFC are thought to be variables that can moderate the relationship between values and environmental behavioural intentions. This is another piece of the puzzle and follows up from the experiments presented in Chapter 4 by including and testing another layer of identity (moral identity instead of group and national identity), as well as adding to previous findings on the relationships between values and environmental engagement. The present study proposes the moderation model depicted in Figure 5.1. The particular moderation role of each of the four variables is discussed in more detail below.

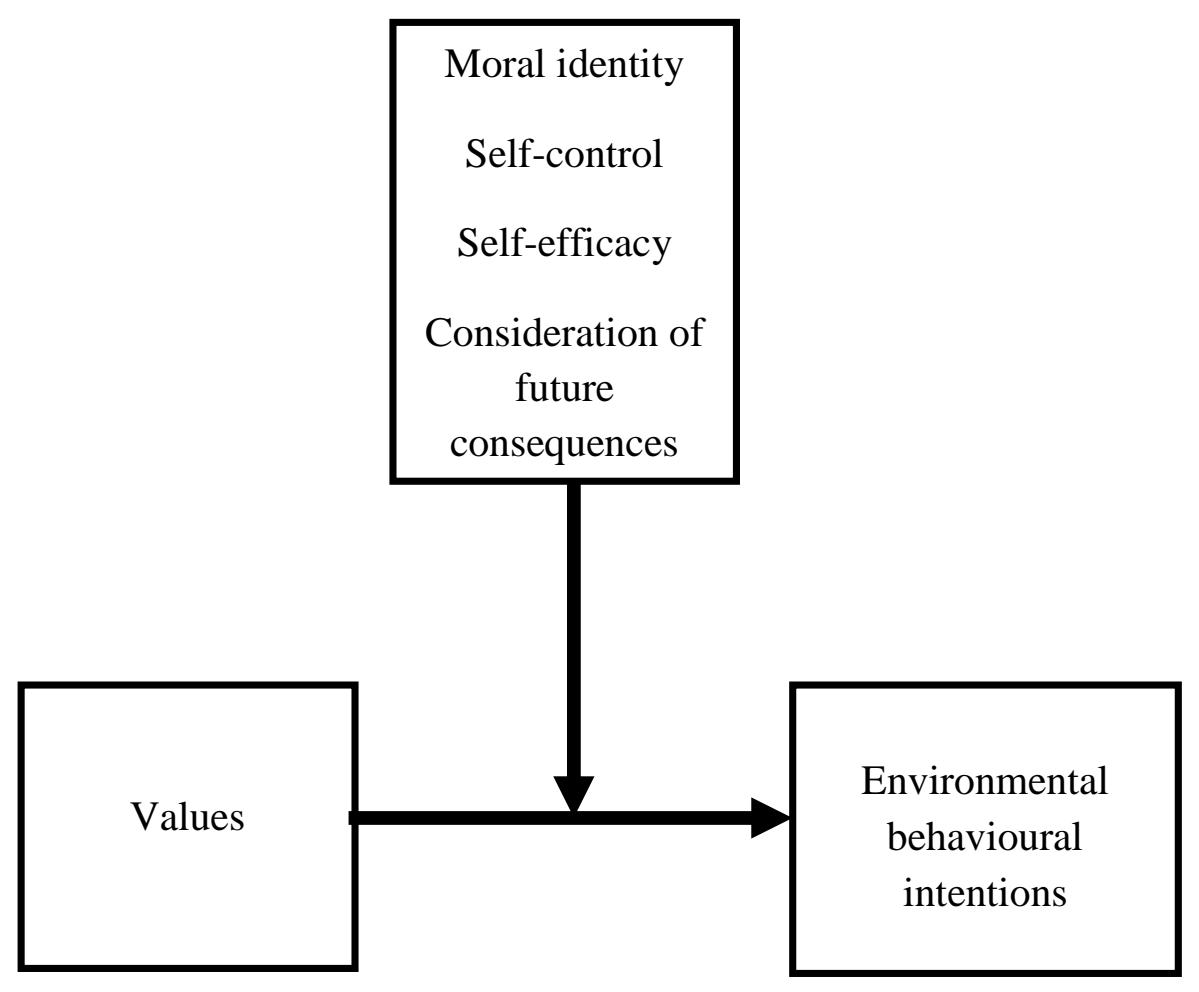

Figure 5.1. Model depicting the proposed conditional effect of values on environmental behavioural intentions moderated by moral identity, self-control, self-efficacy and CFC. 


\section{Moral Identity as Moderator of the Values-Environmental Behavioural Intentions Link}

The two experimental studies in Chapter 4 demonstrated the role of group and national identity in changing values and how the value change affects environmental behavioural intentions. In those studies, the focus was on student identity and national identity (i.e., identification with being a university student or a New Zealander). The findings from both experiments revealed that value change was more likely to occur for those with stronger student or national identity. The present study expands these findings by specifically focusing on moral identity as a possible moderator of the link between values and environmental behavioural intentions.

Moral identity is important in examining the influence of values on environmental engagement because this type of identity is commonly associated with the personal values that individuals hold (Fritsche et al., 2012). In addition, studies have shown that identity (specifically self-identity) influences environmental engagement directly (for reviews, see Nigbur et al., 2010; Whitmarsh \& O'Neill, 2010) and also mediates the relationship between values and both attitudes and behaviours towards the environment (e.g., Dono, Webb, \& Richardson, 2010; Fielding et al., 2008). More importantly, previous studies have shown that moral identity is a good predictor of behavioural intentions (see meta-analysis conducted by Bamberg \& Möser, 2007), pro-social behaviour and environmentalism (Hardy et al., 2014).

Besides these observed direct and mediational effects, it is possible that moral identity also moderates the relationship between values and environmental outcomes. Moral identity might moderate this relationship because individuals may only consider acting in a proenvironmental way if, based on their motivational values, they believe the action is morally important. As discussed at the beginning of this chapter, moral identity has been defined as the identification of individuals with moral traits that they recognise as central and are widely shared in their culture (Aquino \& Reed, 2002; Reed \& Aquino, 2003). Hart (2005) argues that moral identity is important for understanding both the specificity of moral life and the roots of moral failure. Furthermore, a study conducted by Hardy et al. (2014) has shown that moral identity (conceptualised in their study as 'moral ideal self') positively predicted altruism and environmentalism and negatively predicted aggression among adolescents. Their findings also showed how important moral identity is to understand morally relevant adolescent outcomes. In the present study, moral identity is operationalised as participants' ratings of the importance of moral traits to self (e.g., hardworking; Aquino \& Reed, 2002). To 
the best of the author's knowledge, no previous study has assessed whether moral identity can act as a moderator of the relationship between values and environmental behavioural intentions. The present study aims to examine this possibility.

\section{Self-control as Moderator of the Values- Environmental Behavioural Intentions Link}

Another variable that could moderate the influence of values on environmental behavioural intentions is self-control. Self-control has been defined as the "capacity for altering one's own responses, especially to bring them into line with standards such as ideals, values, morals, and social expectations, and to support the pursuit of long-term goals" (Baumeister, Vohs, \& Tice, 2007, p. 351). In short, self-control refers to people's capacity to rule over their own responses (Baumeister \& Exline, 1999), which is related to willpower or the ability to control impulses, act morally, display initiative, and behave according to reasonable choices (Baumeister \& Tierney, 2011).

To date, no study has directly examined how self-control can influence individuals' decisions to engage in environmental actions. However, other studies have shown that the sense of control has important implications for such actions (Aitken, Chapman, \& McClure, 2011; Hines et al., 1986/1987). In their meta-analysis, for example, Hines et al. (1986/1987) observed that internal locus of control (i.e., individual's ability to rely on internal resources as the major determinants of performance) is associated with environmental attitudes and ecological behaviours. The influence of locus of control on the relationship between environmental attitudes and behaviours has also been shown in the context of the Theory of Planned Behaviour (TPB; e.g., Nigbur et al., 2010). Interestingly, a meta-analytical review by Bamberg and Moser (2007) has also shown that, besides moral norms, attitudes and behavioural control predict pro-environmental behavioural intentions.

Although most of the literature on self-control has focused on the importance of this variable for clinical conditions such as psychopathic personality traits (Beaver, Vaughn, \& DeLisi, 2013), delinquency (Boisvert, Wright, Knopik, \& Vaske, 2012; Beaver, Shutt, Boutwell, Ratchford, Roberts, \& Barnes, 2009), and gambling (Beaver, Hoffman, Shields, Vaughn, DeLisi, \& Wright, 2010), the studies briefly reviewed above suggested that selfcontrol can also be an important variable in understanding pro-environmental actions (e.g., Bamberg \& Moser, 2007) and it is reasonable to think that self-control can moderate the relationship between values and environmental behavioural intentions. This is because people might feel more motivated to act in a pro-environmental way if they believe they have the 
control to act and resist formed counterproductive habits. This process is linked to TPB where perceived control plays an important role in moderating the relationship between intention and behaviour (Ajzen, 1991). Before environmental acts become automatic, the need to control the first impulse of gaining pleasure is important to define the performance on pro-environmental tasks. For example, the pleasure associated with having a long hot bath after a day's work has to be controlled and substituted by a more environmentally friendly option (e.g., having a quick shower). Given that people act towards the environment based on their personal motivations or values, self-control could work in combination with values in influencing the environmental outcome, moderating the link between values and environmental behavioural intentions.

\section{Self-efficacy as Moderator of the Values- Environmental Behavioural Intentions Link}

Another potential moderator of the relationship between values and environmental behavioural intentions is self-efficacy. This variable is closely related to self-control, and it refers to "beliefs in one's capabilities to execute the competencies needed to exercise control over events that affect one's welfare" (Bandura, 1986, p. 1), or the belief that one is capable of performing a certain behaviour (Heath \& Gifford, 2006).

Tabernero and Hernandez (2011) noted that few studies have investigated the role of self-efficacy in pro-social and altruistic behaviours, including environmental engagement (for which a quantifiable reward is either not received or received after a substantial delay). However, a small number of studies have shown that attitudes and behaviours towards the environment are enhanced by participants' sense of self-efficacy (Homburg \& Stolberg, 2006; Milfont, 2012; Park \& Yang, 2012; Rabinovich, Morton, Postmes, \& Verplanken, 2009; Tabernero \& Hernandez, 2011). For example, Tabernero and Hernandez showed that self-efficacy predicted environmentally responsible behaviour in the form of recycling, especially when this relationship was mediated by intrinsic motivation - defined in their study as the "desire to expend effort based on interest in and enjoyment of the task itself" ( $p$. 660). Another study that attested to the direct effect of self-efficacy on environmental engagement was conducted by Park and Yang (2012), who found that self-efficacy was associated with intention to participate in environmental activities.

Traditionally, self-efficacy has been used as a mediator between environmental stressors (e.g., pollution) and problem-solving coping (Homburg \& Stolberg, 2006), or as a main predictor of intentions towards recycling (Tabernero \& Hernandez, 2011). While little is 
known about its role in moderating the relationship between values and environmental behavioural intentions, similarly to self-control, it is still possible that self-efficacy might moderate the values-attitudes link. This is because, when personal values are made salient, people might feel more likely to engage in pro-environmental acts if they feel that they have the competency to perform efficiently or effectively in that way. So the link between someone's values and their environmental behavioural intentions are strengthened by their perceived self-efficacy.

\section{Consideration of Future Consequences as Moderator of the Values- Environmental Behavioural Intentions Link}

Finally, the present study will also consider the CFC as a moderator of the relationship between values and environmental behavioural intentions. Previous studies have shown that time perspective (people's ideas of present, past and future as well as their evaluation of the consequences of their acts on a temporal scale) has a strong influence on environmentally responsible attitudes and acts (e.g., Corral-Verdugo, Fraijo-Sing, \& Pinheiro, 2006; Hendrickx, Poortinga, \& van der Kooij, 2001; Milfont et al., 2012; Strathman et al., 1994). Specifically, people's contemplation of the results of their behaviour guides a specific pattern of action towards the environment. For example, a recent meta-analysis conducted by Milfont et al. (2012) showed that future time perspective (i.e., people's prospect of their lives in the future) predicts participant's environmental behaviour more strongly than other dimensions of psychological time (i.e., past-present).

In particular, CFC refers to "the extent to which people consider the potential distant outcomes of their current behaviours and the extent to which they are influenced by these potential outcomes" (Strathman et al., 1994, p. 743). This construct has been largely studied in relation to environmental engagement (for a review see Milfont et al., 2012). For example, Strathman et al. (1994) observed that high scores on CFC predicted stronger opposition towards offshore oil drilling. Other studies that focused on the effect of CFC on environmental actions have shown that future-oriented individuals prefer to commute by public transportation (Joireman, Van Lange, \& Van Vugt, 2004) and conserve more water (Corral-Verdugo et al., 2006). Research findings also supported the relationship between CFC and self-control (Joireman et al., 2008; Ouellette, Hessling, Gibbons, Reis-Bergan, \& Gerrard., 2005; Strathman et al., 1994) and between CFC and SE values (Urien \& Kilbourne, 2011a). 
Like other variables included in the present study (i.e., identity and self-efficacy), no previous research has investigated the moderational effect of CFC. In fact, most of the studies assessing CFC have focused on its direct effect on health and environmental behaviour such as water conservation practices (Corral-Verdugo et al., 2006; Strathman et al., 1994), or the mediational relationship between $\mathrm{CFC}, \mathrm{SE}$ values and environmentally responsible consumption behaviour (Urien \& Kilbourne, 2011a). However, future time perspective is an important variable associated with environmental engagement (Milfont et al., 2012). Specifically, CFC strongly influences people's behaviour towards saving more water (CorralVerdugo et al., 2006), and reducing fuel consumption (Joireman et al., 2004). Furthermore, research suggests that to be able to act in an environmentally friendly way, people must not only be motivated by their personal values, but also be aware of the long-term consequences of their actions and place importance to this effect (e.g., Milfont et al., 2012). In this sense, it is reasonable to think that $\mathrm{CFC}$ may also play the role of strengthening or weakening the link between values and environmental behavioural intentions, and therefore CFC might moderate this relationship.

\section{Study Goals and Hypothesis}

This study aimed to investigate how moral identity, self-control, self-efficacy and CFC moderate the association between people's values and their environmental behavioural intentions. As depicted in Figure 5.1, it is expected that the relationship between values and environmental behavioural intentions would differ at different levels of the four proposed moderating variables. Knowing that ST values were the most strongly related to environmental issues, and ST and SE values represent opposite poles of a single motivational value dimension (Schwartz, 1992), the present study focused on a "difference values scores" created as the algebraic difference between ST and SE values (i.e., ST scores minus SE scores). This values scores were called 'pure' ST values scores. According to this calculation, higher "difference values scores" mean stronger ST values and lower "difference values scores" mean lower ST values.

In order to create the 'pure' ST values scores, the items related to environmental issues were removed from the original ST measure to avoid content overlap with the outcome variable. The three excluded items represented the Nature value type in the Universalism value sub-dimension (i.e., "He/She strongly believes that he/she should care for nature", "It is important to him/her to work against threats to the world of nature", and "protecting the 
natural environment from destruction or pollution is important to him/her"). The conceptual definition in terms of motivational goals for this value is preservation of the natural environment (Schwartz et al., 2012). It is expected that 'pure' ST values scores will be strongly associated with environmental behavioural intentions among individuals with (a) high moral identity, (b) high self-control, (c) high self-efficacy, and (d) high CFC (compared with conditions in which these variables are low).

\section{Method}

\section{Sample}

A total of 297 participants in New Zealand started the online survey, but only 221 completed the entire survey. Of those who completed the survey, the majority was female ( $n$ $=159,72 \%)$ and remainder were males $(n=62,28 \%)$. The majority of the participants $(n=$ $172 ; 78 \%$ ) were first year Psychology students participating in the study for course credit, while the remaining participants were from the general population $(n=49 ; 22 \%)$. Their ages ranged from 17 to 59 years old $(M=21.68 ; S D=5.92)$.

\section{Instruments}

The online survey comprised a total of six measures plus socio-demographic questions. The survey was set up on Qualtrics (http://www.qualtrics.com/) and the measures are described below.

\section{General Ecological Behaviour (GEB) Scale}

The present study used the same adapted version of the GEB used in the two experiments described in Chapter 4. The GEB items were updated as intentional items and answered on a 5-point Likert scale ranging from 0 (not at all willing) to 4 (extremely willing). Although good psychometric indicators of reliability were found in previous studies using this scale, another pilot study was conducted to ensure the items were understandable and the response scale was adequate. Five post-graduate Psychology students read the 50 items of the GEB, and changes were made in the wording of the sentences where they found any lack of clarity. Also, a "not applicable" option was included in the response scale. This was relevant because some items did not accurately reflect the participants' reality. For example, in previous use of this scale some participants did not have a car and so found it difficult to answer items like "I drive my car into or around the city". Following the research by Kaiser 
and Wilson (2004) and studies described in Chapter 4, the GEB was scored as a onedimensional measure of environmental intentions $(\alpha=.94)$.

\section{Portrait of Values Questionnaire (PVQ)}

The PVQ (Schwartz et al., 2012) is the most recent measure to assess the values dimensions originally covered by the Schwartz Value Survey. It consists of 56 items answered on a 6-point Likert scale ranging from 1 (not like me at all) to 6 (very much like me). The items can be arranged into 19 individual value-types: Dependability, Caring, Concern, Nature, Tolerance, Thought, Action, Stimulation, Hedonism, Achievement, Dominance, Resources, Face, Personal, Societal, Tradition, Rules, Interpersonal, and Humility. These 19 value-types are then organised into the 10 value sub-dimensions: Benevolence (Dependability and Caring); Universalism (Concern, Nature, and Tolerance); Self-direction (Thought and Action); Stimulation, Hedonism, Achievement, Power (Dominance and Resources); Face; Security (Societal and Personal); Tradition and Conformity (Interpersonal, Rules, and Humility). The sub-dimensions can then be organised into the four higher order dimensions: Self-transcendence (ST; Benevolence and Universalism); Self-enhancement (SE; Achievement and Power); Openness to Change (Selfdirection and Stimulation); and Conservation (Conformity and Tradition). In the original study, Schwartz et al. (2012) obtained Cronbach's alphas of .71 for ST, .78 for SE, .67 for Openness to Change and .69 for Conservation values dimension. In the present study, the Cronbach's alpha for each one of these four higher order dimensions were $.80, .80, .79$, and, .80 , respectively.

\section{Moral Identity Scale (MIS)}

The Moral Identity Scale developed by Aquino and Reed (2002) assesses a general dimension entitled moral identity which is defined as a self-conception linked to moral traits. The scale has a total of 13 items, including: "It would make me feel good to be a person who has these characteristics" and "I am actively involved in activities that communicate to others that I have these characteristics". Responses are given on a 7-point Likert scale ranging from 1 (strongly disagree) to 7 (strongly agree). The overall Cronbach's alpha for the MIS in the study by Aquino and Reed (2002) was .74. For the present study the scale reported an overall Cronbach's alpha of .70. 


\section{Self-Efficacy Scale (SES)}

The self-efficacy scale, proposed by Schwarzer and Jerusalem (1995), is composed of 10 items measuring a general sense of perceived self-efficacy which is defined as an optimistic self-belief that one can perform a task or cope with adversity. Examples of items are "I can always manage to solve difficult problems if I try hard enough" and "I am confident that I could deal efficiently with unexpected events". The items are answered using a 4-point Likert scale that ranges from 1 (not at all ) to 4 (completely true). Internal reliability (Cronbach's alpha) for the original scale was .80. The Cronbach's alpha for the present study was .86 .

\section{Brief Self-Control Scale (BSCS)}

Composed of 13 items, the Brief Self-Control Scale (BSCS) was originally developed by Tangney, Baumeister, and Boone (2004) after an extensive review of published studies on self-control processes and failures. It is a trait scale measuring individuals' capacity to manage their lives, control their temper, keep their diets, fulfil their promises, stop drinking, save money, persevere at work, keep secrets, and so forth. The items are answered on a 5point Likert scale ranging from 1 (not at all) to 5 (very much). An example of an item is "I am good at resisting temptation”. The average total Cronbach's alpha for this measure reported by Tangney et al. (2004) was .84 . The Cronbach's alpha for the present sample was .61.

\section{Consideration of Future Consequences (CFC) Scale}

Originally proposed by Strathman et al. (1994), the CFC scale contains 12 items measuring the extent to which people consider distant versus immediate consequences of potential behaviours. The items for this original scale are answered on a 5-point Likert scale ranging from 1 (extremely uncharacteristic) to 5 (extremely characteristic). The average total Cronbach's alpha for this original measure was .82. The present study used an expanded version of this scale proposed by Joireman et al. (2012), comprising 14 items measuring two dimensions: future and immediate (see also Arnocky, Milfont, \& Nicol, 2014). The CFC future dimension measures individuals' concern about future consequences (items 1, 2, 6, 7, $8,13,14$; e.g., "I consider how things might be in the future, and try to influence those things with my day to day behaviour"; $\alpha=.80$ ), and the CFC immediate dimension assesses individuals' concern about immediate consequences (items 3, 4, 5, 9, 10, 11, 12; e.g., "I only 
act to satisfy immediate concerns, figuring the future will take care of itself"; $\alpha=.84$ ). The items for the CFC version proposed by Joireman et al. (2004) are answered on a 7-point Likert scale that goes from 1 (very uncharacteristic of me) to 7 (very characteristic of me). The CFC can be considered as integrating two dimensions, but also as one single dimension. In the present study the CFC scale was considered as one single dimension and obtained an overall Cronbach's alpha of .85 .

\section{Procedure}

The study was advertised on the university homepage and students signed up online to take part in an online survey. The study was also advertised on social media (i.e., Facebook) and participants had direct access to the survey link. The survey took approximately 30 minutes to complete. A debriefing statement was presented to the participants at the end of the survey. See Appendices O, P and Q for the full questionnaire.

\section{Analysis}

Preliminary analyses included bivariate correlations and independent samples t-tests to investigate the relationship between demographic variables (i.e., gender, age, and type of values) and the dependent measure of environmental behavioural intentions. Next, the correlations among the variables of interest were examined. Three-step hierarchical regression analyses were performed with environmental behavioural intentions as the dependent variable. All analysis followed the same procedure. The control variables were entered in step 1 (i.e., gender, age, and type of values), the single predictors were entered in step 2 (i.e., 'pure' ST values scores and each of the moderator variables), and the interaction terms between 'pure' ST values scores and each of the moderator variables were entered at step 3. ModGraph (Jose, 2013) was used to enter significant interaction effects and simple slope analyses were conducted.

\section{Results}

\section{Correlations among the Variables of Interest}

Bivariate correlations indicated that environmental behavioural intentions were positively related to ST values (see Table 5.1 below), while showing a negative relationship with SE values. As expected, the 'pure' ST values scores was positively related to 
environmental behavioural intentions. All moderation variables, except self-efficacy, had a significant positive correlation with environmental behavioural intentions. 
Table 5.1

Descriptive Statistics and Correlations

\begin{tabular}{|c|c|c|c|c|c|c|c|c|c|c|c|}
\hline & $M$ & $S D$ & Alpha & 1 & 2 & 3 & 4 & 5 & 6 & 7 & 8 \\
\hline 1. Self-transcendence values & 4.74 & 0.59 & .80 & 1 & .06 & $.57 * * *$ & $.48 * * *$ & $.30 * * *$ & $.31 * * *$ & $.49 * * *$ & $.30 * * *$ \\
\hline 2. Self-enhancement values & 3.74 & 0.76 & .80 & & 1 & $-.76^{* * *}$ & $.20 * * *$ & $.14 *$ & $.17^{* *}$ & .01 & $-.12^{\dagger}(p=.06)$ \\
\hline 3. Pure ST values scores & 1.19 & 0.94 & - & & & 1 & $.12^{\dagger}(p=.08)$ & .06 & .06 & $.27 * * *$ & $.25^{* * *}$ \\
\hline 4. Moral identity & 5.09 & 0.75 & .70 & & & & 1 & $.24 * * *$ & $.33 * * *$ & $.40^{* * *}$ & $.14^{*}$ \\
\hline 5. Self-control & 4.38 & 0.75 & .61 & & & & & 1 & $.42 * * *$ & $.43 * * *$ & $.15^{* *}$ \\
\hline 6. Self-efficacy & 3.09 & 0.44 & .86 & & & & & & 1 & .38 & .04 \\
\hline $\begin{array}{l}\text { 7. Consideration of future } \\
\text { consequences }\end{array}$ & 4.98 & 0.89 & .85 & & & & & & & 1 & $.21 * * *$ \\
\hline 8. GEB & 3.60 & 0.50 & .94 & & & & & & & & 1 \\
\hline
\end{tabular}

Note: $* p<.05 ; * * p<.01 ; * * * p<.001 ;{ }^{\dagger} \mathrm{p}<.10$. 


\section{Relationships between Demographic Variables and the Dependent Measure}

Bivariate correlations revealed that environmental behavioural intentions were not significantly correlated with age. Independent samples t-tests were calculated to examine group differences in environmental behavioural intentions based on gender $(1=$ female and 2 $=$ male $)$ and type of sample $(1=$ undergraduate students and $2=$ general population $)$. While analysis yielded no significant group differences based on gender $(p>.05)$, a significant group difference based on sample type was found, $t(219)=2.27, p<.05$, Cohen's $d=.39$. Undergraduate students scored higher $(M=3.73, S D=0.40)$ in the environmental behavioural intentions measure compared to participants from the general population $(M=$ $3.55, S D=0.52)$. Although age and gender were not associated with environmental behavioural intentions, these variables could still influence the relationships between values and environmental behavioural intentions. Therefore, age, gender and sample type were included as control variables in the regression analysis reported below.

\section{Moderated regression analysis and simple slopes calculations}

It was hypothesised that moral identity, self-efficacy, self-control, and CFC would moderate the relationship between ST values (specifically the 'pure' ST values scores) and environmental behavioural intentions. To test this effect, moderated multiple regressions were conducted with z-scores ${ }^{10}$ of each of the variables. The product term was created by multiplying the z-scores of the 'pure' ST values with each of the moderator variables (moral identity, self-control, self-efficacy, and CFC). The regressions also controlled for age, gender and type of sample (i.e., first year students or general population). Four sets (one for each moderator) of three-step hierarchical regression analysis were performed with environmental behavioural intentions as the dependent variable. All analyses followed the same procedure. The control variables were entered in step 1, the single predictors were entered in step 2, and the interaction at step 3 . The results of the regression models are presented in Table 5.2 below.

\footnotetext{
${ }^{10}$ Standardised z-scores were used to standardise scores of predictors that were measured on different scales, facilitating the interpretation of the results and graphs. Centred scores could also have been used; although they are not the same as z-scores, it is very likely they would yield similar results. Furthermore, according to Jose (2013), centring is not an essential step and the analysis with or without centring produces a pattern that is identical in both cases.
} 
Table 5.2 Hierarchical Regression of the Prediction of Environmental Behavioural Intentions

\begin{tabular}{|c|c|c|c|c|c|c|c|c|c|c|c|c|}
\hline & \multicolumn{3}{|c|}{ Step 1} & \multicolumn{3}{|c|}{ Step 2} & \multicolumn{3}{|c|}{ Step 3} & \multicolumn{3}{|c|}{ Step 4} \\
\hline & $\Delta R^{2}$ & $\beta$ & $t$ & $\Delta R^{2}$ & $B$ & $t$ & $\Delta R^{2}$ & $\beta$ & $t$ & $\Delta R^{2}$ & $\beta$ & $t$ \\
\hline \multicolumn{13}{|l|}{ Moral Identity } \\
\hline Gender & 0.054 & 0.06 & 0.85 & 0.046 & 0.012 & 0.18 & 0.010 & 0.002 & 0.036 & $0.020 *$ & -0.004 & -0.054 \\
\hline Age & & -0.17 & $-2.28^{*}$ & & -0.18 & $-2.49^{* *}$ & & -0.17 & $-2.43^{*}$ & & -0.17 & $-2.32 *$ \\
\hline Sample Type & & -0.22 & $-2.95^{* *}$ & & -0.19 & $-2.68 * *$ & & -0.19 & $-2.62 * *$ & & -0.18 & $-2.58 * *$ \\
\hline Pure ST values & & & & & 0.22 & $3.31^{* * *}$ & & 0.21 & $3.16^{* *}$ & & 0.19 & $2.81^{* *}$ \\
\hline Moral identity & & & & & & & & 0.10 & 1.51 & & 0.11 & $\begin{array}{c}1.68^{\dagger} \\
(\mathrm{p}=.09)\end{array}$ \\
\hline Pure ST values X Moral Identity & & & & & & & & & & & 0.14 & $2.18 *$ \\
\hline \multicolumn{13}{|l|}{ Self-efficacy } \\
\hline Gender & 0.054 & 0.06 & 0.85 & 0.046 & 0.012 & 0.18 & 0.000 & 0.013 & 0.20 & 0.001 & 0.015 & 0.223 \\
\hline Age & & -0.17 & $-2.28 *$ & & -0.18 & $-2.49 * *$ & & -0.18 & $-2.49 * *$ & & -0.18 & $-2.49 * *$ \\
\hline Sample Type & & -0.22 & $-2.95 * *$ & & -0.19 & $-2.68 * *$ & & -0.19 & $-2.63 * *$ & & -0.19 & $-2.59 * *$ \\
\hline Pure ST values & & & & & 0.22 & $3.31 * * *$ & & 0.22 & $3.28^{* * *}$ & & 0.22 & $3.25 * * *$ \\
\hline Self-efficacy & & & & & & & & 0.02 & 0.26 & & 0.01 & 0.21 \\
\hline Pure ST values X Self-efficacy & & & & & & & & & & & -0.03 & -0.44 \\
\hline \multicolumn{13}{|l|}{ Self-control } \\
\hline Gender & 0.054 & 0.06 & 0.85 & 0.046 & 0.012 & .18 & 0.019 & 0.025 & 0.38 & 0.004 & 0.028 & .42 \\
\hline Age & & -0.17 & $-2.28^{*}$ & & -0.18 & $-2.49 * *$ & & -0.20 & $-2.74 * *$ & & -0.20 & $-2.78 * *$ \\
\hline Sample Type & & -0.22 & $-2.95 * *$ & & -0.19 & $-2.68 * *$ & & -0.18 & $-2.48 * *$ & & -0.18 & $-2.47 * *$ \\
\hline Pure ST values & & & & & 0.22 & $3.31 * * *$ & & 0.21 & $3.23^{* * *}$ & & 0.21 & $3.21 * *$ \\
\hline Self-control & & & & & & & & 0.14 & $2.13^{*}$ & & 0.15 & $2.12^{*}$ \\
\hline Pure ST values X Self-control & & & & & & & & & & & -0.06 & -0.94 \\
\hline \multicolumn{13}{|l|}{ CFC } \\
\hline Gender & 0.054 & 0.06 & 0.85 & 0.046 & 0.012 & 0.18 & 0.019 & 0.014 & 0.211 & 0.005 & 0.011 & 0.160 \\
\hline Age & & -0.17 & $-2.28^{*}$ & & -0.18 & $-2.49 * *$ & & -0.20 & $-2.72 * *$ & & -0.19 & $-2.66 * *$ \\
\hline Sample Type & & -0.22 & $-2.95 * *$ & & -0.19 & $-2.68 * *$ & & -0.17 & $-2.34 *$ & & -0.17 & $-2.36^{*}$ \\
\hline Pure ST values & & & & & 0.22 & $3.31^{* * *}$ & & 0.19 & $2.77 * *$ & & 0.17 & $2.40^{*}$ \\
\hline CFC & & & & & & & & 0.14 & $2.13^{*}$ & & 0.14 & 1.13 \\
\hline Pure ST values X CFC & & & & & & & & & & & 0.07 & 1.13 \\
\hline
\end{tabular}

Note: $* p<.05 ; * * p<.01 ; * * * p<.001,{ }^{\dagger} p<.10$ 
Moral Identity. The three-step regression model explained $10 \%$ of variance in environmental behavioural intentions; $F(6,212)=5.23, p<.001, R^{2}=0.13, \operatorname{Adj} R^{2}=0.10$, $R^{2}$ change $=0.02$ (see Table 5.2). In the final step, age of the participants $(\beta=-0.17, t=-$ $2.32, p<.05)$ and type of the sample $(\beta=-0.18, t=2.58, p<.01)$ showed a significant main effect. In addition, 'pure' ST values scores also showed a significant main effect $(\beta=0.19, t$ $=2.81, p<.01)$. Furthermore, a marginally significant main effect of moral identity $(\beta=0.11$, $t=1.68, p<.10$ ) was found. Regarding the interactions (see Table 5.2), the analyses showed the predicted significant interaction between the 'pure' ST values scores and moral identity in predicting environmental behavioural intentions $(\beta=0.14, t=2.18, p<.05)$, suggesting that the strength of the relationship between values and environmental behavioural intentions was affected by moral identity. This confirmed the hypothesis that moral identity moderates the relationship between 'pure' ST values scores and environmental behavioural intentions.

To model the significant 'pure' ST values scores vs. moral identity interaction, simple effects were calculated. They represented the mean differences of environmental behavioural intentions across 'pure' ST values at low (1 SD below the mean), medium and high (1 SD above the mean) values of moral identity (see Figure 5.2). 


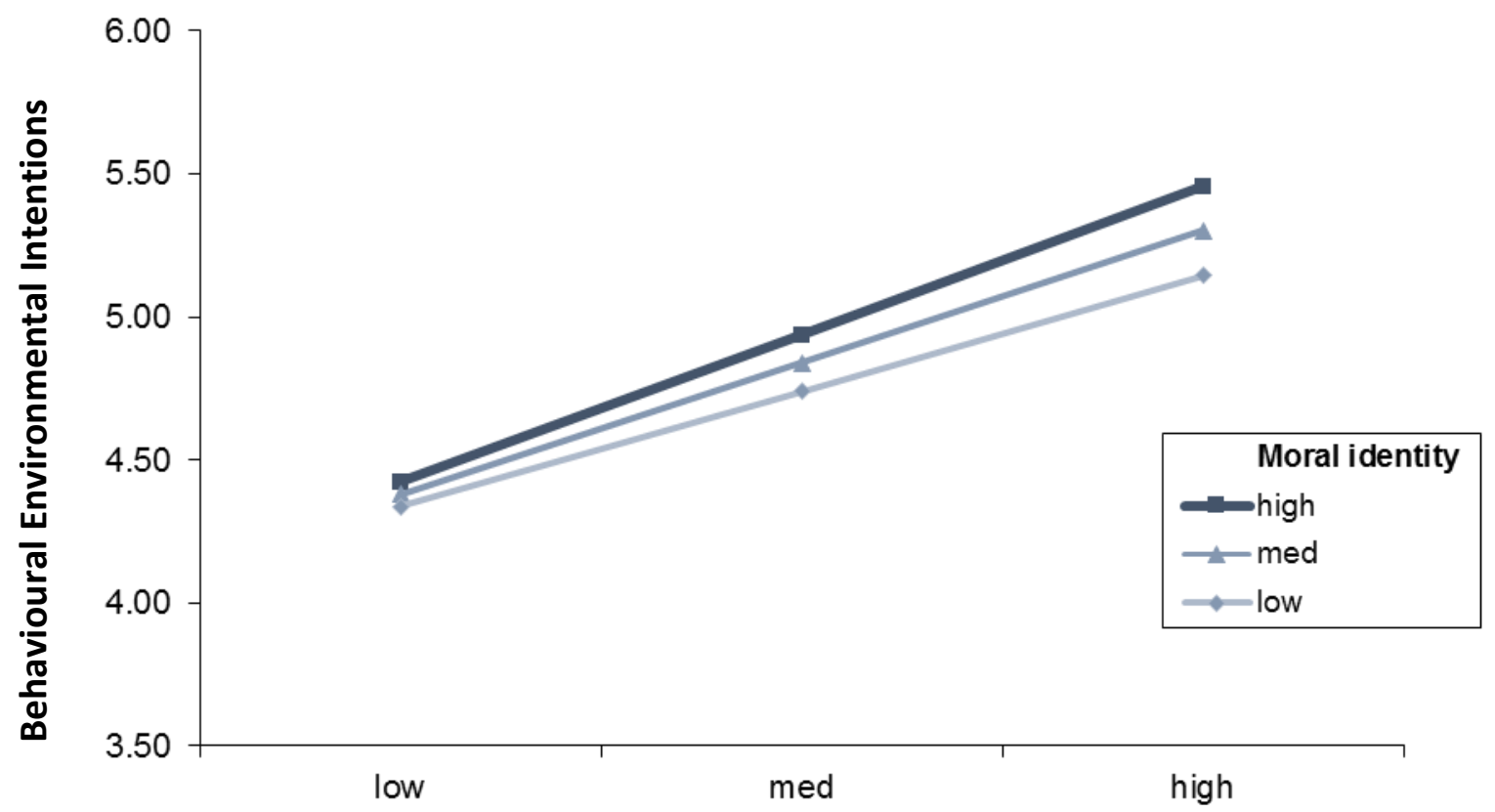

Pure ST values scores

Figure 5.2. The levels of moral identity enhanced the positive effects of 'pure' selftranscendence values scores on environmental behavioural intentions (moderators included separately in four different sets of analyses).

Simple slopes analyses revealed that the association between 'pure' ST values and environmental behavioural intentions was stronger for those participants with high moral identity $($ simple slope $=0.53, t(216)=2.69, p<.001)$. Analyses also revealed that the relationship between 'pure' ST and environmental behavioural intentions was weaker for participants with low moral identity (simple slope $=0.42, t(216)=2.85, p<.001$ ). Additionally, this association was also significantly affected by medium levels of moral identity ( simple slope $=0.47, t(216)=2.72, p<.001)$. These results confirmed the hypotheses that moral identity would moderate the relationship between values and environmental behavioural intentions, and that 'pure' ST values scores would be strongly associated with environmental behavioural intentions among individuals with high levels of 
moral identity. In other words, moral identity enhances the positive influence of 'pure' ST values on environmental intentions.

Self-efficacy. The three-step model was significant and explained $7 \%$ of the variance in environmental behavioural intentions; $F(6,212)=3.97, p<.001, R^{2}=0.10, \operatorname{Adj} R^{2}=0.07$, $\mathrm{R}^{2}$ change $=0.001$. In the final step, a significant main effect of age $(\beta=-0.18, t=-2.49, p$ $<.01)$ and sample type $(\beta=-0.19, t=-2.59, p<.01)$ was found. Also, 'pure' ST values scores showed a significant main effect $(\beta=0.22, t=3.25, p<.001)$, although there was no significant main effect of self-efficacy $(\beta=0.01, t=0.211, p>.05)$. No significant interaction was found between 'pure' ST values scores and self-efficacy $(\beta=-0.03, t=-0.44$, $p>.05)$ and the hypothesis that self-efficacy moderates the relationship between 'pure' ST values scores and environmental behavioural intentions was not confirmed.

Self-control. The three-step regression model explained $9 \%$ of variance in environmental behavioural intentions; $F(6,212)=4.92, p<.001, R^{2}=0.12$, Adj $R^{2}=.09, \mathrm{R}^{2}$ change $=0.004$. The control variables of age $(\beta=-0.20, t=-2.79, p<.01)$ and sample type $(\beta=-0.18, t=-2.47, p<.01)$ showed a significant main effect. A significant main effect was also found for 'pure' ST values scores $(\beta=0.21, t=3.21, p<.01)$ and self-control $(\beta=0.15$, $t=2.21, p<.05)$. However, there was no significant interaction between 'pure' ST values scores and self-control $(\beta=-0.06, t=-0.94, p>.05)$. Consequently, the hypothesis that selfcontrol moderates the link between 'pure' ST values scores and environmental behavioural intentions was not confirmed.

CFC. The three-step regression model explained $9 \%$ of variance in environmental behavioural intentions; $F(6,212)=4.99, p<0.001, R^{2}=0.12$, Adj $R^{2}=0.09, \mathrm{R}^{2}$ change $=$ 0.005. Although the results yielded a main effect of age of the participants $(\beta=-0.19, t=$ $2.66, p<.01)$, type of sample $(\beta=-0.17, t=-2.36, p<.01)$, 'pure' ST values scores $(\beta=.17$, $t=2.40, p<.01)$ and CFC $(\beta=.14, t=2.08, p<.05)$, no significant interaction between 'pure' ST values scores and CFC $(\beta=0.08, t=1.13, p>.05)$ was found. Thus, the hypothesis that CFC would act as a moderator of the relationship between 'pure' ST values scores and environmental behavioural intentions was not confirmed. 


\section{Examining the robustness of the moral identity moderation}

To examine the extent to which the significant 'pure' ST values scores vs. moral identity interaction is robust another regression analysis was performed including control variables, single predictors and interaction terms in three different steps. Results of the regression model are presented in Table 5.3 below.

Table 5.3

Hierarchical Regression of the Prediction of Environmental Behavioural Intentions Combining All Moderator Variables

\begin{tabular}{|c|c|c|c|c|c|c|c|c|c|}
\hline & \multicolumn{3}{|c|}{ Step 1} & \multicolumn{3}{|c|}{ Step 2} & \multicolumn{3}{|c|}{ Step 3} \\
\hline & $\Delta R^{2}$ & $\beta$ & $t$ & $\Delta R^{2}$ & $\beta$ & $t$ & $\Delta R^{2}$ & $\beta$ & $t$ \\
\hline Gender & 0.04 & 0.05 & 0.71 & 0.13 & -0.01 & -0.01 & $0.16^{*}$ & 0.008 & 0.123 \\
\hline Age & & -0.14 & $-1.94 *$ & & -0.18 & $-2.62 * *$ & & -0.19 & $-2.59 * *$ \\
\hline Sample Type & & -0.17 & $-2.43^{*}$ & & -0.14 & $-2.05^{*}$ & & -0.17 & $-2.33^{*}$ \\
\hline Pure ST values & & & & & -0.23 & $-3.46 * * *$ & & 0.15 & $2.21 *$ \\
\hline Moral identity & & & & & 0.03 & 0.47 & & 0.07 & 0.96 \\
\hline Self-efficacy & & & & & -0.06 & -0.87 & & -0.068 & -0.92 \\
\hline Self-control & & & & & 0.14 & $\begin{array}{c}1.85^{\dagger}(\mathrm{p} \\
=.06)\end{array}$ & & 0.10 & 1.26 \\
\hline CFC & & & & & 0.07 & 0.87 & & 0.09 & 1.26 \\
\hline Pure ST values X Moral Identity & & & & & & & & 0.15 & 2.019* \\
\hline Pure ST values X Self-efficacy & & & & & & & & -0.001 & -0.01 \\
\hline Pure ST values X Self-control & & & & & & & & -0.14 & $\begin{array}{c}-1.80^{\dagger}(\mathrm{p} \\
=.07)\end{array}$ \\
\hline Pure $S T$ values $X$ CFC & & & & & & & & 0.04 & 0.49 \\
\hline
\end{tabular}

Note: $* p<.05 ; * * p<.01 ; * * * p<.001,^{\dagger} p<.10$.

The model was significant and explained $11 \%$ of variance in environmental behavioural intentions; $F\left(12,206=3.27, p<.001, R^{2}=0.16\right.$, Adj $R^{2}=0.11, R^{2}$ change $=$ $0.16, p<.001$. The results presented in Table 5.3 mirrored the previous findings. There was a significant main effect of age $(\beta=-0.19, t=-2.59, p<.01)$ and sample type $(\beta=-0.17, t=-$ 2.33, $p<.05)$. Additionally, a main effect for 'pure' ST values scores was also found $(\beta=$ $0.15, t=2.21, p<.05)$. No other variables yielded a significant main effect. The analyses also revealed a significant interaction between 'pure' ST values scores and moral identity $(\beta=$ $0.15, t=2.019, p<.05)$. In addition, a marginally significant interaction was found between 'pure' ST values scores and self-control $(\beta=-0.14, t=-1.80, p<.10)$, while all the other 
interaction terms remained non-significant (self-efficacy $\beta=-.001, t=-0.01, p>.05$, and CFC $\beta=0.04, t=0.49, p>.05)$. These results thus confirm the moderation of moral identity discussed above. To model this significant interaction, simple slopes were again calculated (see Figure 5.3 below).

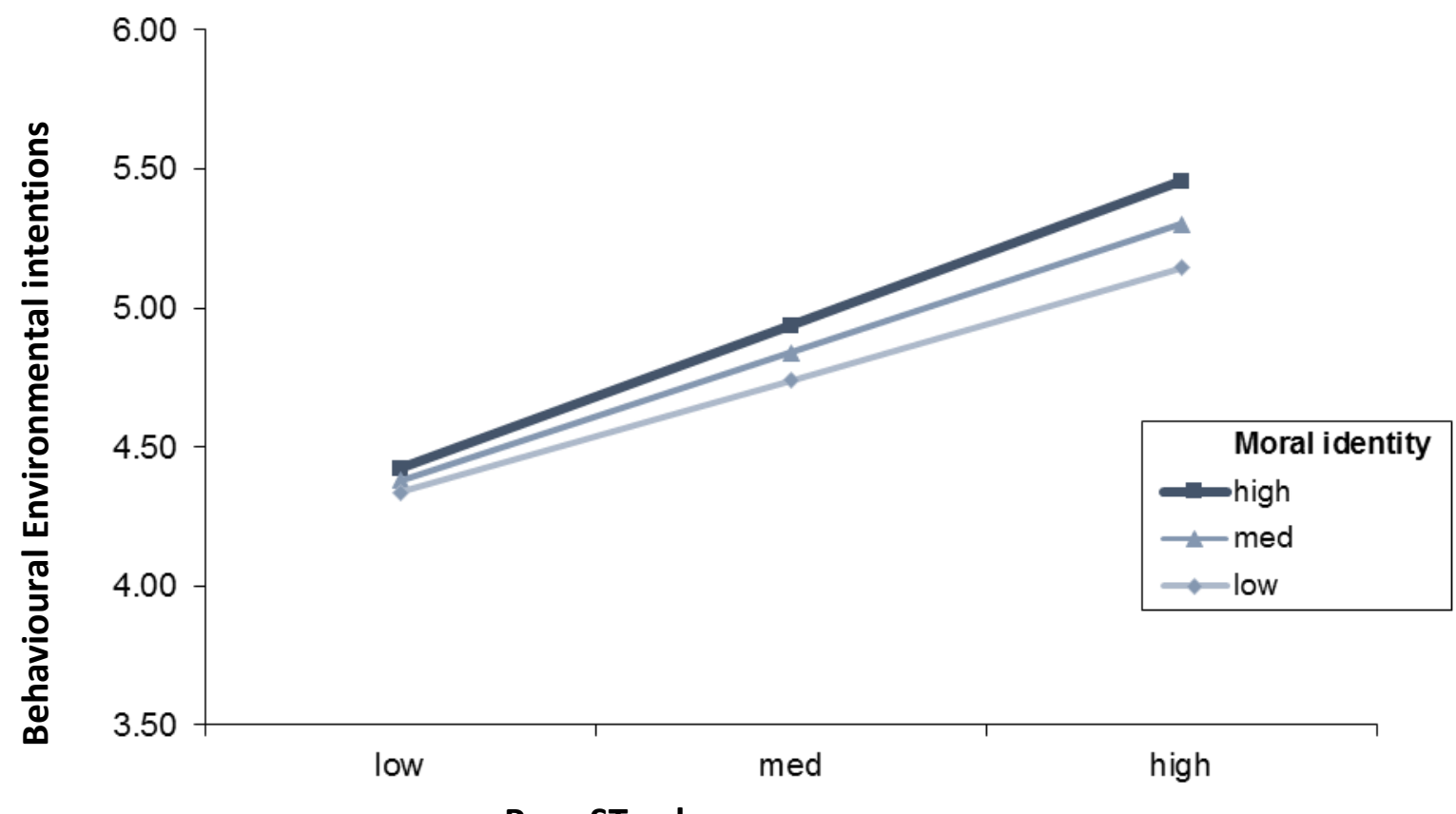

Pure ST values scores

Figure 5.3. The levels of moral identity enhanced the positive effects of 'pure' selftranscendence values scores on environmental behavioural intentions (moderators included simultaneously in one set of analysis).

According to Figure 5.3, simple slopes mirrored those reported in the previous analysis: the relationship between a high orientation towards 'pure' ST values and environmental behavioural intentions was stronger for those participants with high moral identity ( simple slope $=0.55, t(216)=2.35, p<0.01$ ), and the association was weaker for participants with low moral identity (simple slope $=0.43, t(216)=2.45, p<0.01)$.

Additionally, the relationship between 'pure' ST values and environmental behavioural intentions was also significantly affected by medium levels of moral identity (simple slope $=$ $0.49, t(216)=2.39, p<0.001)$. These results confirmed that the interaction is robust even after including other variables in the model. In other words, moral identity acts as an enhancer of the positive effect of 'pure' ST values on environmental behavioural intentions. 


\section{Additional regression analysis}

Further moderation analyses were conducted considering ST and SE values separately as predictors to examine whether distinct interactions would be observed, compared to when considering only the 'pure' ST values scores. No specific hypotheses were proposed when ST and SE were analysed separately. However, based on previous results using the 'pure' ST values scores, it was expected that only moral identity would moderate the relationship between ST values and environmental behavioural intentions. It was also expected that no significant moderation would be observed for SE values in predicting environmental behavioural intentions. To test these effects, similar moderated multiple regressions were conducted with z-scores of each one of the variables following the same procedure as the previous analysis using 'pure' ST values scores. The results for ST and SE values are shown in Tables 5.4 and 5.5, respectively. 
Table 5.4

Hierarchical Regression of the Prediction of Environmental Behavioural Intentions for Self-transcendence Values

\begin{tabular}{|c|c|c|c|c|c|c|c|c|c|c|c|c|}
\hline & \multicolumn{3}{|c|}{ Step 1} & \multicolumn{3}{|c|}{ Step 2} & \multicolumn{3}{|c|}{ Step 3} & \multicolumn{3}{|c|}{ Step 4} \\
\hline & $\Delta R^{2}$ & $\beta$ & $t$ & $\Delta R^{2}$ & $\beta$ & $t$ & $\Delta R^{2}$ & $\beta$ & $t$ & $\Delta R^{2}$ & $\beta$ & $t$ \\
\hline \multicolumn{13}{|l|}{ Moral Identity } \\
\hline Gender & 0.054 & 0.06 & 0.85 & 0.035 & 0.019 & 0.27 & 0.002 & 0.017 & 0.25 & 0.012 & 0.02 & 0.36 \\
\hline Age & & -0.17 & $-2.28 *$ & & -0.17 & $-2.31 *$ & & -0.17 & $-2.28 *$ & & -0.17 & $-2.28^{*}$ \\
\hline Sample Type & & -0.22 & $-2.95^{* *}$ & & -0.19 & $-2.65^{* *}$ & & -0.19 & $-2.65^{* *}$ & & -0.19 & $-2.62 * *$ \\
\hline ST values & & & & & 0.19 & $2.85 * *$ & & 0.17 & $2.31 *$ & & 0.16 & $2.18^{*}$ \\
\hline Moral identity & & & & & & & & 0.05 & 0.68 & & 0.07 & 0.916 \\
\hline ST values X Moral Identity & & & & & & & & & & & 0.11 & $1.70^{\dagger}(p=.09)$ \\
\hline Gender & 0.054 & 0.06 & 0.85 & 0.035 & 0.019 & 0.27 & 0.001 & 0.043 & .20 & 0.008 & 0.021 & 0.311 \\
\hline Age & & -0.17 & $-2.28 *$ & & -0.17 & $-2.31^{*}$ & & -0.16 & $-2.25^{*}$ & & -0.17 & $-2.30^{*}$ \\
\hline Sample Type & & -0.22 & $-2.95 * *$ & & -0.19 & $-2.65^{* *}$ & & -0.20 & $-2.68 * *$ & & -0.20 & $-2.70^{*}$ \\
\hline ST values & & & & & 0.19 & $2.85^{* *}$ & & 0.20 & $2.85^{* *}$ & & 0.19 & $2.64 * *$ \\
\hline Self-efficacy & & & & & & & & -0.03 & -0.48 & & -0.54 & 0.58 \\
\hline ST values X Self-efficacy & & & & & & & & & & & -0.09 & -1.35 \\
\hline \multicolumn{13}{|l|}{ Self-control } \\
\hline Gender & 0.054 & 0.06 & 0.85 & 0.035 & 0.019 & 0.27 & 0.010 & 0.034 & 0.49 & 0.013 & 0.03 & 0.45 \\
\hline Age & & -0.17 & $-2.28 *$ & & -0.17 & $-2.31^{*}$ & & -0.18 & $-2.49^{* *}$ & & -0.19 & $-2.56^{* *}$ \\
\hline ST values & & & & & 0.19 & $2.85^{* *}$ & & 0.16 & $2.33^{*}$ & & 0.17 & $2.42 *$ \\
\hline Self-control & & & & & & & & 0.11 & 1.53 & & 0.11 & 1.55 \\
\hline ST values X Self-control & & & & & & & & & & & -0.11 & $-1.76^{\dagger}(p=.08)$ \\
\hline \multicolumn{13}{|l|}{ CFC } \\
\hline Gender & 0.054 & 0.06 & 0.85 & 0.035 & 0.019 & 0.27 & 0.014 & 0.026 & 0.37 & 0.001 & 0.024 & 0.355 \\
\hline Age & & -0.17 & $-2.28 *$ & & -0.17 & $-2.31^{*}$ & & -0.18 & $-2.53 * *$ & & -0.18 & $-2.50 * *$ \\
\hline Sample Type & & -0.22 & $-2.95 * *$ & & -0.19 & $-2.65^{* *}$ & & -0.17 & $-2.39^{*}$ & & -0.17 & $-2.37 * *$ \\
\hline ST values & & & & & 0.19 & $2.85 * *$ & & 0.14 & $1.90^{*}$ & & 0.14 & $1.92 *$ \\
\hline $\mathrm{CFC}$ & & & & & & & & 0.13 & $1.80^{\dagger}(\mathrm{p}=0.07)$ & & 0.13 & $1.75^{\dagger}(\mathrm{p}=.08)$ \\
\hline ST values X CFC & & & & & & & & & & & 0.023 & 0.35 \\
\hline
\end{tabular}

Note: ${ }^{*} p<.05 ; * * p<.01 ; * * * p<.001,{ }^{\dagger} p<.10$. 


\section{Table 5.5}

Hierarchical Regression of the Prediction of Environmental Behavioural Intentions for Self-enhancement Values

\begin{tabular}{|c|c|c|c|c|c|c|c|c|c|c|c|c|}
\hline & \multicolumn{3}{|c|}{ Step 1} & \multicolumn{3}{|c|}{ Step 2} & \multicolumn{3}{|c|}{ Step 3} & \multicolumn{3}{|c|}{ Step 4} \\
\hline & $\Delta R^{2}$ & $\beta$ & $t$ & $\Delta R^{2}$ & $\beta$ & $t$ & $\Delta R^{2}$ & $\beta$ & $t$ & $\Delta R^{2}$ & $\beta$ & $t$ \\
\hline \multicolumn{13}{|l|}{ Moral Identity } \\
\hline Gender & 0.054 & 0.06 & 0.85 & 0.012 & 0.048 & 0.70 & 0.021 & 0.028 & 0.408 & 0.006 & 0.02 & 0.26 \\
\hline Age & & -0.17 & $-2.28 *$ & & -0.18 & $-2.39 *$ & & -0.17 & $-2.36^{*}$ & & -0.17 & $-2.28 *$ \\
\hline Sample Type & & -0.22 & $-2.95^{* *}$ & & -0.21 & $-2.91 * *$ & & -0.21 & $-2.81 *$ & & -0.20 & $-2.79 * *$ \\
\hline SE values & & & & & -0.11 & $-1.68^{\dagger}(\mathrm{p}=.09)$ & & -0.14 & $-2.13 *$ & & -0.13 & $-1.92 *$ \\
\hline Moral identity & & & & & & & & 0.15 & $2.20^{*}$ & & 0.14 & $2.11^{*}$ \\
\hline SE values X Moral Identity & & & & & & & & & & & -0.08 & -1.19 \\
\hline \multicolumn{13}{|l|}{ Self-efficacy } \\
\hline Gender & 0.054 & 0.06 & 0.85 & .012 & 0.048 & 0.70 & 0.002 & 0.051 & 0.75 & 0.003 & 0.05 & 0.728 \\
\hline Age & & -0.17 & $-2.28^{*}$ & & -0.18 & $-2.39 *$ & & -0.18 & $-2.44 * *$ & & -0.18 & $-2.47 * *$ \\
\hline Sample Type & & -0.22 & $-2.95^{* *}$ & & -0.21 & $-2.91 * *$ & & -0.21 & $-2.80 * *$ & & -0.21 & $-2.86^{* *}$ \\
\hline SE values & & & & & -0.11 & $-1.68^{\dagger}(\mathrm{p}=.09)$ & & -0.12 & $-1.79^{\dagger}(\mathrm{p}=.07)$ & & -0.19 & $-1.74^{\dagger}(\mathrm{p}=.08)$ \\
\hline Self-efficacy & & & & & & & & 0.05 & 0.73 & & 0.05 & 0.78 \\
\hline SE values X Self-efficacy & & & & & & & & & & & -0.06 & -0.84 \\
\hline \multicolumn{13}{|l|}{ Self-control } \\
\hline Gender & 0.054 & 0.06 & 0.85 & .012 & 0.048 & 0.70 & 0.028 & 0.06 & 0.90 & 0.0004 & 0.06 & 0.87 \\
\hline Age & & -0.17 & $-2.28 *$ & & -0.18 & $-2.39 *$ & & -0.20 & $-2.73 * *$ & & -0.20 & $-2.72 * *$ \\
\hline Sample Type & & -0.22 & $-2.95^{* *}$ & & -0.21 & $-2.91 * *$ & & -0.19 & $-2.66^{* *}$ & & -0.19 & $-2.66^{* *}$ \\
\hline SE values & & & & & -0.11 & $-1.68^{\dagger}(\mathrm{p}=.09)$ & & -0.14 & $2.09^{*}$ & & 0.14 & $-2.10^{*}$ \\
\hline Self-control & & & & & & & & 0.17 & $2.55^{* *}$ & & 0.17 & $2.50^{* * *}$ \\
\hline SE values X Self-control & & & & & & & & & & & -0.02 & -0.30 \\
\hline \multicolumn{13}{|l|}{ CFC } \\
\hline Gender & 0.054 & 0.06 & 0.85 & .012 & 0.048 & 0.70 & 0.035 & 0.04 & 0.61 & 0.011 & 0.03 & 0.50 \\
\hline Age & & -0.17 & $-2.28^{*}$ & & -0.18 & $-2.39 *$ & & -0.20 & $-2.74 * *$ & & -0.20 & $-2.69 * *$ \\
\hline Sample Type & & -0.22 & $-2.95^{* *}$ & & -0.21 & $-2.91 * *$ & & -0.18 & $-2.42 *$ & & -0.18 & $2.49 * *$ \\
\hline SE values & & & & & -0.11 & $-1.68^{\dagger}(\mathrm{p}=.09)$ & & -0.12 & $-1.85^{\dagger}(\mathrm{p}=.06)$ & & -0.09 & -1.39 \\
\hline CFC & & & & & & & & 0.19 & $2.88 * *$ & & 0.18 & $2.69 * *$ \\
\hline SE values X CFC & & & & & & & & & & & -0.11 & -1.64 \\
\hline
\end{tabular}

Note: $* p<.05 ; * * p<.01 ; * * * p<.001,{ }^{\dagger} p<.10$ 
The results presented in Tables 5.4 and Table 5.5 are summarised below.

Moral identity: The results in Table 5.4 revealed that the three-step model significantly explained $8 \%$ of variance in environmental behavioural intentions; $F(6,212)=$ 4.04, $p<0.001, R^{2}=0.10$, Adj $R^{2}=0.08, R^{2}$ change $=0.012$. In the final step, age $(\beta=-0.17$, $t=-2.28, p<.05)$ and sample type $(\beta=-0.19, t=-2.62, p<.01)$ showed significant main effects. In addition, ST values also showed a significant main effect $(\beta=0.16, t=2.18, p$ $<.05)$. No main effect was found for moral identity. Regarding interactions, the analysis revealed a marginally significant interaction between ST values and moral identity in predicting environmental behavioural intentions $(\beta=0.11, t=1.70, p<.10)$. Although the interaction was not significant, this result followed the predicted positive direction and the pattern observed in previous analysis when the 'pure' ST values scores were considered.

In turn, Table 5.5 shows that the three-step model for SE values explained $7 \%$ of variance in environmental behavioural intentions; $F(6,212)=3.63, p<.001, R^{2}=0.09$, Adj $R^{2}=0.067, R^{2}$ change $=0.006$. In the final step, age $(\beta=-0.17, t=-2.28, p<.05)$ and sample type $(\beta=-0.20, t=-2.79, p<.01)$ showed significant main effects. In addition, SE values $(\beta$ $=-0.13, t=-1.92, p<.05)$ and moral identity $(\beta=0.14, t=2.11, p<.05)$ also showed significant main effects. As expected, no interaction was found between SE values and moral identity.

Self-efficacy. The three-step model for ST values reported in Table 8 significantly explained $7 \%$ of the variance in environmental behavioural intentions; $F(6,212)=3.80, p<$ $0.001, R^{2}=0.10$, Adj $R^{2}=0.07, R^{2}$ change $=0.008$. In the final step, age $(\beta=-0.17, t=-2.30$, $p<.05)$ and sample type $(\beta=-0.20, t=-2.70, p<.01)$ showed significant main effects. In addition, ST values also showed a significant main effect $(\beta=0.19, t=2.64, p<.01)$. Neither a main effect for self-efficacy, nor an interaction between ST values and self-efficacy were found, similarly to the previous analysis using the 'pure' ST values scores.

The three-step model for SE values reported in Table 5.5 significantly explained $4 \%$ of the variance in environmental behavioural intentions; $F(6,212)=2.731, p<0.001, R^{2}=$ 0.07 , Adj $R^{2}=0.045, R^{2}$ change $=0.003$. In the final step, age $(\beta=-0.18, t=-2.47, p<.01)$ and sample type $(\beta=-0.21, t=-2.86, p<.01)$ showed significant main effects. In addition, SE values showed a marginally significant main effect $(\beta=-0.19, t=-1.74, p<.10)$. As expected, neither main effect for self-efficacy, nor interaction between SE values and selfefficacy were found. 
Self-control. The three-step model for ST values (see Table 5.4) significantly explained $8 \%$ of the variance in environmental behavioural intentions; $F(6,212)=4.43, p$ $<.001, R^{2}=0.11$, Adj $R^{2}=0.08, R^{2}$ change $=0.013$. In the final step, age $(\beta=-0.19, t=-$ $2.56, p<.01)$ and sample type $(\beta=-0.19, t=-2.57, p<.01)$ showed significant main effects. In addition, $\mathrm{ST}$ values also showed a significant main effect $(\beta=0.17, t=2.42, p<.01)$. A main effect for self-control was not found and the interaction between ST values and selfcontrol was only marginally significant $(\beta=-0.11, t=-1.76, p<.10)$. This result somewhat followed previous findings observed when the 'pure' ST values scores were considered.

The three-step model for SE values (see Table 5.5) significantly explained $7 \%$ of the variance in environmental behavioural intentions; $F(6,212)=3.68, p<.001, R^{2}=0.09$, Adj $R^{2}=0.069, R^{2}$ change $=0.000$. In the final step, age $(\beta=-0.20, t=-2.72, p<.01)$ and sample type $(\beta=-0.19, t=-2.66, p<.01)$ showed significant main effects. A significant main effect was also found for SE values $(\beta=0.14, t=-2.10, p<.05)$ and self-control $(\beta=0.17, t=2.50$, $p<.01)$. As expected, the interaction between SE values and self-control was not significant.

CFC. The three-step model for ST values depicted in Table 5.4 significantly explained $8 \%$ of the variance in environmental behavioural intentions; $F(6,212)=4.03, p$ $<.001, R^{2}=0.10$, Adj $R^{2}=0.08, R^{2}$ change $=0.001$. In the final step, age $(\beta=-0.18, t=-$ $2.50, p<.01)$ and sample type $(\beta=-0.17, t=-2.37, p<.05)$ showed significant main effects. In addition, there was a significant main effect for ST values $(\beta=0.14, t=1.92, p<.05)$, while CFC showed a marginally significant main effect $(\beta=0.13, t=1.75, p<.10)$. No interaction between ST values and CFC was found, mirroring previous results using 'pure' ST values scores.

The three-step model for SE values depicted in Table 5.5 significantly explained 9\% of the variance in environmental behavioural intentions; $F(6,212)=4.479, p<.001, R^{2}=$ 0.112 , Adj $R^{2}=0.087, R^{2}$ change $=0.011$. In the final step, age $(\beta=-.20, t=-2.69, p=.008)$ and sample type $(\beta=-0.18, t=2.49, p<.01)$ showed significant main effects. Although a main effect was not found for SE values, a significant main effect was found for CFC $(\beta=$ $0.18, t=2.69, p<.01)$. As expected, no interaction between SE values and CFC was found.

Overall the moderated regression results examining the ST and SE values scores separately partially replicated the results for the 'pure' ST values scores. Moral identity interacted (although marginally) with ST values in predicting environmental behavioural intentions, with no other moderator variables showing a significant interaction with ST or SE 
values. A marginally significant interaction between ST values and self-control was also observed, and could be explored in further studies.

\section{Discussion}

The main purpose of this study was to examine whether moral identity, self-control, self-efficacy, and CFC moderate the relationships between values and environmental behavioural intentions. Four main hypotheses were tested regarding each one of the four moderators but only one was confirmed. Only moral identity significantly moderated the relationship between values and environmental behavioural intentions, confirming the first hypothesis. Also in line with the first hypothesis, moral identity enhanced the positive effects of 'pure' ST values on environmental behavioural intentions. It was observed that the effect of the 'pure' ST values scores on environmental behavioural intentions is particularly strong when participants score high in moral identity. This finding reinforces the role of identity in understanding the associations between values and environmental behavioural intentions. Perhaps even more importantly, this finding highlights the significance of considering moral identity as a moderator and not only as a main predictor or mediating variable of environmental behavioural intentions (Dono et al., 2010; Fielding et al., 2008; Nigbur et al., 2010; Whitmarsh \& O’Neill, 2010).

The finding that moral identity moderates the link between values and environmental behavioural intentions is also consistent with previous findings showing that moral identity and values are strongly and positively related, due to the nature of these two constructs (Fritsche et al., 2012). This finding also supports previous studies linking morality and prosocial behaviour, including environmental behaviour (De Groot \& Steg, 2009; 2010). Furthermore, this finding is also complementary to research that suggests a strong effect of ST values on environmental outcomes (see both previous empirical chapters of this thesis; Boer \& Fischer, 2013; Karp, 1996; Milfont, Sibley \& Duckitt, 2010; Schultz \& Zelezny, 1999; Shultz et al., 2005; Stern et al., 1995) and expands results from the two experiments in Chapter 4 by highlighting the importance of moral identity (and not only student and national identity) and ST values on promoting environmentally friendly behavioural intentions.

However, it is important to highlight that although moral identity was a significant moderator of the relationship between values and environmental behavioural intentions, this result was only confirmed when 'pure' ST values scores were included in the model. When the ST values scores were examined separately, only a marginally significant interaction was 
observed. A possible explanation for this partially contradictory yet interesting finding is that the ends of the higher order continuum representing the ST-SE dimension are 'contaminated' by the influence of the opposed extreme of the dimension. Since ST and SE values fall at opposite ends of a single dimension, the ST values scores are influenced by the SE values scores, and vice-versa. When analyses are conducted with the 'pure' ST values scores, the influence caused by SE values on the scores of the participants on ST values is removed. Although the correlations for the 'pure' ST values scores are weaker overall compared to those for the ST values scores (see Table 5.1), this 'pure' score produced a more powerful variable for detecting interactions. It is also possible that when the SE values influence is removed, the resulting 'pure' ST values scores is more closely related to morality. Previous studies have attested to the links between morality and values (Vauclair, 2010) and between morality and environmental engagement (De Groot \& Steg, 2009; 2010) and it is possible that, in the present study moral identity has a strong relation with the ST values and environmental behavioural intentions due to the morality content assumed by the 'pure' ST values scores.

Interestingly, moral identity was the only moderator in this study to significantly strengthen the relationship between values and environmental behavioural intentions. The second hypothesis stated that individuals with strong self-control and high scores on the 'pure' ST values scores would show more environmental behavioural intentions; while the third hypothesis proposed that people with strong self-efficacy and high scores on 'pure' ST values would show more environmental behavioural intentions. Neither of these hypotheses was confirmed. One possible explanation for these results is that the participants had already previously formed habits and therefore self-control and self-efficacy had a small or null impact on the link between values and environmental behavioural intentions. It might also be useful to think differently about the influence that behavioural control has as a moderator between values, attitudes, intentions and consequently, behaviours. Although research reinforces the mechanism proposed by TPB, which includes behavioural-control as a moderator of the intention-behaviour link (Aitken et al., 2011; Bamberg \& Moser, 2007; Hines et al., 1986/1987), the results presented here suggest that behavioural control might not moderate the effect of antecedent variables on intention. On the other hand, environmental behavioural intentions was assessed through a broader measure of general pro-environmental intentions. It might be the case that behavioural control influences the link between values and only some types of intentions towards the environment but not others. For example, behavioural control would 
exert stronger influence on intentions that represent behaviours that are easier to perform and do not involve too much risk, such as saving water (see work by Kaiser \& Wilson, 2004).

The fourth and final hypothesis predicted that people with high scores on CFC and high scores on ST values would show more environmental behavioural intentions. This was not confirmed. The future time perspective assessed by the CFC did not moderate the valuesenvironmental behavioural intentions link in the present study, contradicting previous research findings that has shown that people who are more concerned with the future tend to be more environmentally friendly (Diniz, Wilson, \& Milfont, 2010; Milfont et al., 2012). The present findings also go against previous studies (Urien \& Kilbourne, 2011) that have shown that $\mathrm{SE}$ values had a positive effect (instead of a negative effect) on environmental behavioural intentions when associated with consideration of impacts of environmental issues in future generations. Although Urien and Kilbourne (2011) attested that people who strongly believe in leaving their legacy in the future endorse their SE values differently (i.e., interpreting that looking out for one's self means being concerned for the welfare of others), non-significant results were found in the present study when SE values were considered separately in the analysis. In sum, the relationships between CFC and SE values should be investigated further to address the difference between the present results and those presented by Urien and Kilbourne (2011). Future research could also investigate how CFC could be used to target values (maybe even SE values instead of ST values) and promote environmental behavioural intentions.

\section{Limitations}

This study represents one of the first attempts to propose a moderation model of individual variables, values and environmental behavioural intentions. However, while this study is important as an attempt to better understand what strengthens the link between values and intentions to act in an environmentally friendly way, it also has some limitations.

As pointed out by other researchers (e.g., Gifford, 2008), the relationship between values and environmental behavioural intentions is complex, and there are many other variables that could be considered while studying this link. An example is the effectiveness of public policies and access to facilities such as an efficient recycling system (see Chen \& Tung, 2010). These variables were not considered in the present study but may account for explaining environmental behavioural intentions, especially in the New Zealand context. The limited framework of the present study (only considering four moderators) thus permits a limited, 
although not less relevant, conclusion. Of the four moderators considered, only moral identity yielded a significant influence in the values-environmental behavioural intentions relationship, explaining around $10 \%$ of the variance. Many other variables account for explaining environmental engagement and a broader understanding of the relationship between values and environmental behavioural intentions could be gained by incorporating other variables into the model.

Furthermore, the sample was composed mainly of New Zealanders and mostly first year Psychology students. Consequently, generalizations of the findings might be limited. Furthermore, it would be beneficial to have a more diverse and representative sample from other countries. This would offer a more complete understanding of the factors affecting environmental behavioural intentions in other cultural contexts, and consequently increase the possibility of generalizing the results.

Finally, research has shown that socio-economic status plays a role in environmental engagement (Bond, Leung, Au, Tong, \& Chemonges-Nielson, 2004; Hawcroft \& Milfont, 2010). It is well known that economic factors influence how much people care about the environment (see, e.g., Bond et al., 2004; Sadalla \& Krull, 1995) and it would be reasonable to consider that economic factors may influence the values-environmental behavioural intentions link. This variable was not considered in the present study, but it would be interesting in the future to look specifically at how socio-economic status interacts with values and environmental behavioural intentions. It would be especially interesting to examine these relations with a multi-cultural sample (Bond et al., 2004).

\section{Applications}

This study was designed to extend previous research and not only investigate main effects. A moderation study can be productive in offering a better understanding of the links between normative motives and environmental behavioural intentions. The results of this study can be used to guide social scientists and environmentalists in offering practical solutions that focus on removing potential barriers and increase triggers that can boost environmental behavioural intentions, one of the best predictors of actual behaviour (Ajzen, 1991; Ajzen \& Fishbein, 1980). Moral identity is shown here to be a variable that helps to strengthen the relationships between ST values and environmental behavioural intentions. In this case, ST values can efficiently promote environmental behavioural intentions when combined with moral identity. Programmes aiming to promote environmental outcomes among individuals 
could focus on reinforcing ST values and, at the same time, target people with strong moral identity. Another practical application of the results presented in this chapter comprises the use of media to promote environmental behavioural intentions. Advertisements with the aim to promote environmental related actions could use messages that target ST values (instead of other values) and that at the same time make moral identity salient.

\section{Conclusions}

In conclusion, this study showed the importance of examining moderating factors influencing the values-environmental behavioural intentions link. Results suggested that moral identity moderates the relationship between values and environmental behavioural intentions. In particular, they showed that moral identity enhances the positive influence of ST values on environmental behavioural intentions. This study has also shed light on the ST vs. SE values debate by investigating which extreme of this spectrum is subject to greater effects of moderating variables towards environmental behavioural intentions. This research has contributed to reinforcing the importance of values in promoting environmental behavioural intentions. Finally, it has broadened the horizons about environmental issues as an urgent global topic, and suggested possible solutions to boost environmental behavioural intentions. 


\section{CHAPTER SIX: GENERAL DISCUSSION}

\section{Overview}

This thesis aimed to advance research on values and environmental engagement. In particular, this thesis provided a systematic examination of the link between values and environmental outcomes, investigating the mechanism underlying this process and the influence of individual variables on strengthening this relationship. In three studies, the thesis aimed to answer three main questions: First, what are the main values that predict environmental engagement? Second, can we experimentally manipulate values, thereby promoting value change through identity and impacting environmental behavioural intentions? Third, is the relationship between values and environmental behavioural intentions moderated by moral identity, self-control, self-efficacy, and CFC? This research programme was designed after a careful review, revision, and critical examination of the literature on values and environmental engagement. The findings presented in this thesis have offered new insights into the factors that determine the process of value-change, and have examined what other aspects should be considered within the values-environmental engagement domain.

Whereas each of the preceding chapters served a specific goal, they were all complementary and instrumental for the overall purpose of the thesis. This final chapter pulls a thread through the results of the three studies, summarising the key findings and offering a bigger picture of how the relevant psychological theories and findings of this work are consolidated under the overarching account of values and environmental outcomes. Key findings of these three studies are summarised in this chapter, followed by a discussion around the contribution made to the literature and their practical applications. Following this, the limitations of this research will be summarised and suggestions will be made concerning the direction of future research.

\section{Key Research Findings}

The studies presented in the thesis take novel approaches and use multiple methods in order to build a complex and multifaceted understanding of the relationships between values 
and environmental outcomes. A schematic overview of the main findings from each study is depicted in Figure 6.1. The particular findings are discussed in detail below. 

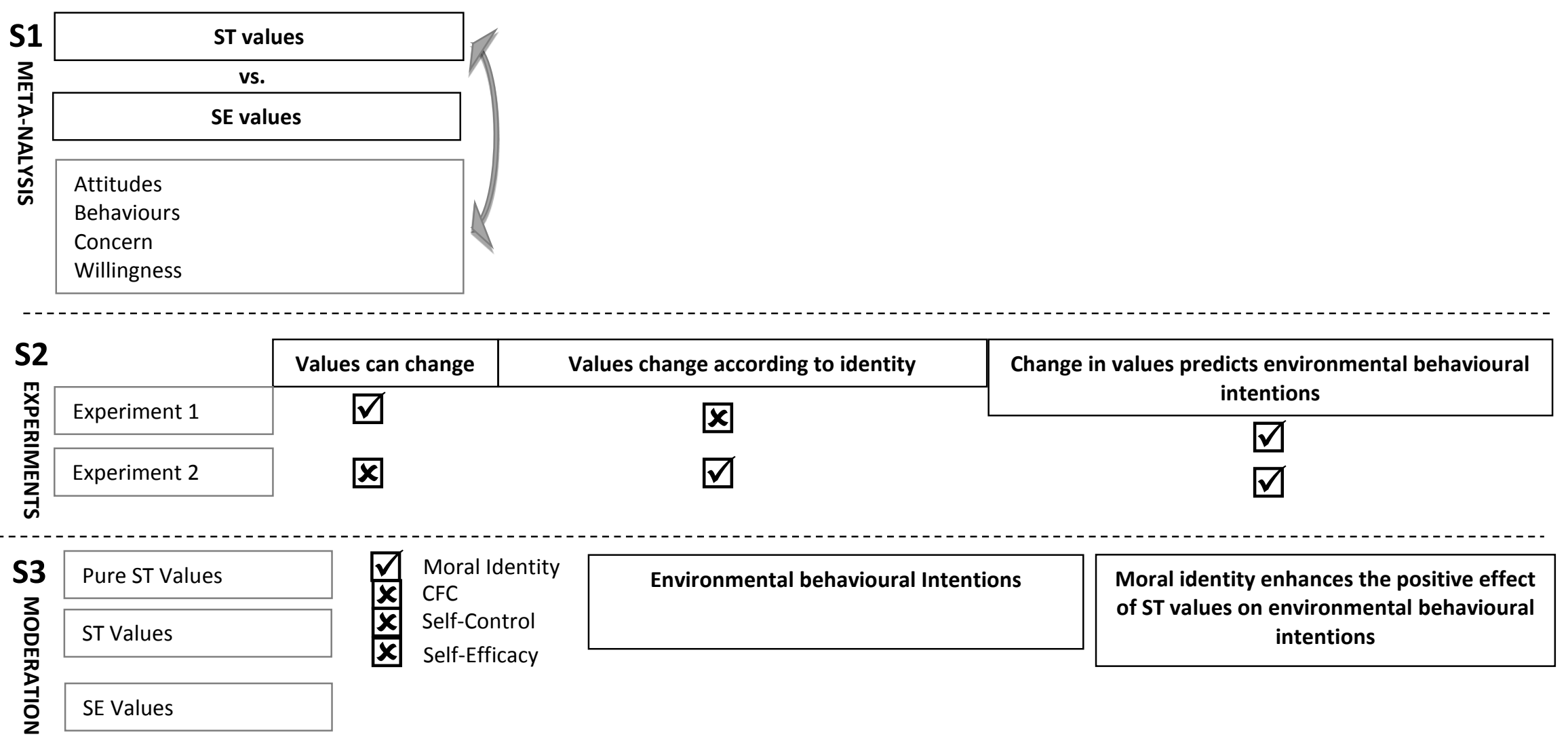

Figure 6.1. Schematic summary of main findings from each study. 


\section{Self-transcendence Values Are the Primary Values Predicting Environmental Outcomes}

The focus of Study 1, presented in Chapter 3, was to gather data from samples across the world to investigate the relationships between values and environmental engagement. To accomplish this, Study 1 used a meta-analytical review of published and unpublished research assessing the relationship between values, specifically ST and SE values, and environmental engagement (i.e., attitude, behaviour, concern and willingness). The meta-analytical review showed that many empirical studies have examined the effects of specific dimensions of values on a variety of environmental outcomes. The final meta-analysis data set was composed of a total of 36 studies, which corresponded to a total sample size of 47,714 participants from 58 countries, indicating that the results are consistent across a broad range of cultures with different characteristics.

The results from Study 1 showed that nine different values measures have been used in the literature to assess values linked to environmental outcomes. It was also shown that many authors have tried to assess and build a more specific theory of environmental values, usually by integrating the environmentally related items from the Schwartz's values measure, offering value dimensions that specifically tap environmental issues (De Groot \& Steg, 2007, 2008; Milfont, Sibley, \& Duckitt, 2010; Schultz, 2001; Stern \& Dietz, 1994; Stern et al., 1993). The Schwartz's values theory and measure was the most common values framework and assessment used in the literature to explain environmental engagement. Furthermore, a diverse number of environmental outcomes have been used in the literature, the main ones being environmental attitudes, behaviours, concern and willingness.

In line with previous findings (e.g., Becker \& Félonneau, 2011; F eather, 2002; Fukukawa et al., 2007; Milfont, Sibley, \& Duckitt, 2010; Schultz \& Zelezny, 1999; Schultz et al., 2005; Stern et al., 1998), the meta-analysis showed that the ST vs. SE values dimension is the most widely examined in the literature and is indeed the best predictor of environmental outcomes. This suggests that the ST vs. SE dimension should be the main focus when examining the effect of values on environmental outcomes. The effect size for ST values and environmental engagement 
was similar in strength to findings from a meta-analysis conducted by Boer and Fischer (2013).

In particular, the results showed a stronger effect size for ST values predicting environmental behaviours, attitudes, concern and willingness compared to SE values predicting these same environmental outcomes. These results are congruent with empirical findings showing that values are better predictors of environmental engagement than other values (e.g., Milfont, Duckitt, \& Wagner, 2010; Schultz et al., 2005). Furthermore, the meta-analysis illustrated the important role of ST values in promoting environmental engagement. This means that people who are more concerned about the welfare and interest of others are more inclined to engage in a proenvironmental way. This finding provides empirical support for the view that it might be strategic to assess ST values in order to promote relevant environmental outcomes, and confirms previous studies that have also attested to the importance of ST values to promote environmental engagement (e.g., Milfont, Sibley, \& Duckitt, 2010; Schultz et al., 2005).

It is interesting to note, however, that although the overarching findings across all studies considered in the meta-analysis pointed towards the importance of the ST vs. SE dimension, other values dimensions might also predict specific environmental outcomes. Although Study 1 did not examine dimensions other than ST and SE values, there are indications in the literature that the openness to change dimension, for example, is a meaningful value dimension specifically related to environmentally friendly actions (Nordlund \& Garvill, 2002). Others have claimed that SE values are not necessarily a negative predictor of environmental engagement (e.g., Schultz et al., 2005). In fact, although most studies showed that SE values negatively predict environmental outcomes, a significant positive relationship was identified in the present meta-analysis between SE values and environmental concern, confirming past research findings by Schultz et al. (2005).

Furthermore, the meta-analytical results showed that contextual effects influence the relationship between values and environmental engagement, especially regarding sample type, the type of values measure used and the type of environmental outcome assessed. In general, the relationship between values and environmental outcomes was stronger for students than for general population samples. Moreover, when the type of values measure was included as a moderator of the relationship between values and 
environmental engagement, the initial analysis showed that the type of values measure influences this relationship. However, a close inspection of the confidence intervals indicates that the associations between ST values and some environmental outcomes are similar across type of values measures (i.e., the Schwartz measure and other's measures). One possible explanation for this result is that many of the other values measures considered consisted of a variation of the Schwartz's values items (e.g., The Brief Inventory of Values; Stern et al., 1998), or they are measures based on theories that converge with the Schwartz's values such as Inglehart's model (e.g., Wilson, 2005). Finally, when moderated analysis were performed with type of environmental outcome as a moderator of the values-environmental behavioural intentions link, results showed that the relationship between ST/SE values is stronger when the environmental measure assessed environmental attitudes compared to environmental behaviours.

As noted above, studies in the meta-analysis included multiple indicators of environmental engagement, including distinct measures of attitudes, behaviours, concern and willingness towards the environment. However, the majority of the studies focused on measuring attitudes and behaviours. Willingness or intentions to act are the important middle ground between our attitudes towards a specific act and what we actively do (Ajzen, 1991) but have received little attention based on studies included in the meta-analysis. Studies 2 and 3 in this thesis focused on environmental behavioural intentions, making an important contribution that helps to fill this gap between attitudes and behaviours.

In sum, the results of the meta-analysis demonstrate the importance of values (especially ST values) in explaining environmental engagement. This supports what was expected (e.g., Schultz et al., 2005) and most importantly highlighted the importance of studying values to understand people's environmental engagement. The social media and public opinion have already suggested the importance of understanding the psychological motivations behind the way that we relate to the environment, and psychological research can be valuable in identifying the ways our values influence our engagement with environmentally friendly acts. The results from Study 1 provided an overview of what has been shown so far on the topic of values and environmental engagement and provided an overall conclusion from the extant literature on the issue which pointed towards ST values explaining people's environmental engagement. 


\section{Values can Change}

The second research question of the thesis was: Can we change people's values and, if so, can this change in values influence people's environmental behavioural intentions? The second study, presented in Chapter 4, aimed to answer this research question by manipulating values in an experimental setting. The study first assessed value change, then examined whether identity has an influence on value change. Finally, the study investigated the influence of value change on environmental behavioural intentions.

A review of the literature revealed that few experimental studies have investigated value change. One reason for this might be based on the argument that values are stable and therefore it should be difficult if not impossible to produce any change experimentally, or to test a long-term effect (Bardi \& Goodwin, 2011; Bardi et al., 2014). However, some previous research findings have shown that it is possible to empirically manipulate values (Maio \& Olson, 1998; Maio et al., 2009; Rokeach, 1973). Furthermore, manipulating values can be especially useful when testing the effectiveness of using particular techniques, such as the VSC technique, to influence the way participants respond to specific constructs like environmental behavioural intentions, in order to better understand important social issues. These points are detailed below.

\section{The VSC technique}

The VSC technique used in the two experiments conducted represent the theoretical and methodological perspective that laid the foundation for designing and interpreting the results. This technique, originally proposed by Rokeach (1973) and extensively used by Maio and colleagues (2009), is based on the assumption that when specific values are activated they will incur changes in values which tap a similar dimension. Maio et al. employed the VSC technique to promote value change using the Schwartz's values. According to Maio et al., the proposed confrontation between values and consequent value change that the VSC produces fits with the model proposed by Schwartz. The results found for both experiments in Chapter 4 attest to the circular dynamic proposed by the Schwartz's values model and advocated by Maio et al. The results of Experiments 1 and 2 showed that when the VSC technique targeted values in the manipulation, what resulted was a change in values at the post-test. Particularly, 
when ST values were primed in the pre-test, both ST and SE values changed in the posttest, so that ST values increased and SE values decreased. In particular, the three-way interaction between time, the type of values and type of condition was significant for Experiment 1, indicating that values serving the same dimension changed in the same direction as the promoted value, whereas values serving opposing dimensions changed in the opposite direction. These findings provide additional empirical support for compatibility and conflict between the value dimensions in the circular values model proposed by Schwartz (1992). These results also suggest that although values are considered stable constructs, they can be changed experimentally (at least temporarily) through the VSC technique. Additionally, it may be also possible that this change on values can last long term, an issue that should be addressed in future research. In sum, the main results from Experiment 1 showed that the VSC technique was successful in eliciting value change.

Interestingly, the findings from Experiment 1 were only partially replicated in Experiment 2 with a general sample. The three-way interaction between time, the type of values and type of condition was not significant; however, the single comparisons in Experiment 2 did show differences in the values of participants from pre- to post-test, between conditions. It could be that university students are more malleable with regard to values than the general population. In fact, the general population sample was older than the university students sample and, although values structures can be found at younger ages (Doring et al., 2015), studies have shown that values are still being formed in youth while values in older aged participants are more stable (e.g., Achenreiner, 1997). Therefore, the age difference between samples might explain the distinct results.

In summary, the two experiments in Study 2 of this thesis demonstrate the dynamic of changing values with the use of the VSC technique. Similar results have been reported by previous studies using the same technique (see Maio et al., 2009; Rokeach, 1973). Across both experiments the results showed that the value change manipulation led to a change in values, particularly in ST values. In a previous study by Maio et al. (2009), ST values were also shown to be the type of values most prone to change. 


\section{The prediction of environmental behavioural intentions}

A final research question answered by Experiments 1 and 2 was: Does change in values influence people's environmental behavioural intentions? In Experiment 1, although value change did not predict a change in environmental behavioural intentions from pre-test to post-test (a decrease in environmental behavioural intentions was observed), value change predicted environmental behavioural intentions measured after the experimental manipulation at the post-test. Similar results were observed in Experiment 2, with change in values not predicting a change in environmental behavioural intentions and a decrease in environmental behavioural intentions. However, once again the change in values predicted environmental behavioural intentions measured at the post-test. Furthermore, in both experiments, changes in ST values were the main component explaining environmental behavioural intentions, at least at the post-test.

This result suggests that systematic value change can influence environmental behavioural intentions, especially when this type of intention is congruent with ST values but conflict with SE values. These results are consistent with previous results by Mayo et al. (2009), Bargh, Gollwitzer, Lee-Chai, Barndollar, and Trotschel (2001), and Macrae and Johnston (1998), which demonstrated an effect of value-change and valuepriming on value-congruent behaviour. However, to the author's knowledge, Study 2 is the first set of experiments to demonstrate the effects of value change on environmental behavioural intentions.

The results presented here also confirm the argument raised by Maio et al. (2009) that when changing or priming values with the purpose of altering value-relevant attitudes and behaviours, researchers should consider indirect effects of prioritised values on non-targeted attitudes and behaviours. As shown by Maio et al.'s findings, and confirmed by the findings in the present thesis, the indirect effects can be predicted by considering the motivational interconnections between values. This also falls in line with a previous study illustrating how a strong effect on environmental behaviours is shown when individuals are primed with ST values, and furthermore that this effect is absent when another conflicting value dimension (SE values) is primed at the same time (Evans et al., 2013). 
In sum, the results from Experiments 1 and 2 showed that value change has an impact on environmental behavioural intentions measured after the experimental manipulation, supporting predictions that ST value change would affect environmental intentions, as they are congruent with these values. Furthermore, the results from both experiments indicated that ST value change has an impact on environmental behavioural intentions. This finding supports the role of ST values in predicting environmental engagement observed in Study 1.

\section{Identity is Important}

Another important topic that Experiments 1 and 2 investigated was what guides the value change. Previous studies have pointed to the importance of social comparison and norm adaptation (e. g., Chernoff \& Davison, 2005), and the research presented in this thesis was premised on the assumption that values change through identity. Two types of identity were investigated in Experiments 1 and 2 - student and national identity. For Experiment 1, although it was not confirmed that values change according to identity, the overall pattern of results based on the single comparisons suggested that ST values are more prone to change among people scoring high in student identity. Most of these findings were replicated in Experiment 2. Although a significant threeway interaction between type of values, time and conditions was not found in Experiment 2 (suggesting no value change), it was found that ST values were more prone to change and change in values was more pronounced among participants with high national identity. Taken as a whole, the two studies revealed insights into the important role of identity in value change.

Importantly, some results counter to predictions emerged in both Experiments 1 and 2, raising some questions about the effective impact of the VSC technique, depending on the reference group used. For example, in Experiment 1, the expected three-way interaction between time, type of condition and student identity was not found. A possible reason for this is that these participants did not identify as fully as a university student, as they are still in their first year of university and potentially have not formed a strong connection with university students yet. However, an overall trend emerged in the expected direction and the manipulation had a greater effect for participants who highly identify with the reference group of university students than with the low identity group. The results suggested that the VSC technique did not work 
as well for the low identity group as it did for the high identity group, suggesting that students who do not identify as strongly with the reference group of university students may not pay much attention to the values preferences of other students. On the other hand, students who do strongly identify as being a VUW student are more prone to the effects of social comparison with the student reference group and so more easily adapt their values accordingly.

Interestingly, the results found for Experiment 1, specifically regarding the high identity score group exposed to the ST condition, were also confirmed in Experiment 2 with a general population sample and reference group of New Zealanders. In Experiment 2, the three-way interaction between time, condition and identity was significant, indicating that New Zealand-born participants were more prone to changing ST values from pre-test to post-test if they strongly identified with being a New Zealander (the reference group in Experiment 2). In fact, the change in ST values was greater for participants with stronger identification with the national group and exposed to the ST condition. However, when participants with high identification with the national group were exposed to the SE condition, their ST (minus SE) values also increased unexpectedly. This counter-intuitive result also differed from Experiment 1, where participants who highly identified with the student reference group exhibited a change of values in the expected direction for both conditions. As noted above, these distinct results could have been due to age differences between the samples. In Experiment 2 participants were older than the participants in Experiment 1, and so, were possibly less easily influenced. They also likely have strongly internalised New Zealand values, and may not have found the experimental material convincing, since SE values are potentially less aligned with the values promoted in New Zealand than ST values (e.g., Podsiadlowski \& Fox, 2011). Another possible explanation is the effect of social desirability in SE values.

Overall, the results from both experiments have given support for the important role of student and national identity when people compare themselves with members of a reference group, and that this process has an impact on the mechanism of changing values motivations which consequently predict environmental behavioural intentions at the post-test. More importantly, it demonstrates that ST values are more prone to change but also that making ST values salient (as results for the ST condition has shown) is more effective for promoting value change than priming SE values (as results for the 
SE condition has shown). This was consistent for both experiments, assessing two different types of identity.

Study 3, presented in Chapter 5, also examined the role of identity in explaining the link between values and environmental behavioural intentions, as well as other potential moderating variables. Based on the previous literature on values and environmental intentions, four potential individual differences variables were considered: moral identity, self-efficacy, self-control, and CFC. Here as well, the Schwartz's values dimensions of ST and SE values were assessed and linked to environmental behavioural intentions. In contrast with the other studies in this thesis, a survey was conducted in Study 3 with a sample of university students and the general population. The main findings showed that only moral identity positively moderated the associations between values and environmental behavioural intentions, confirming previous research that has pointed to the link between moral identity, values (Vauclair, 2010) and environmental outcomes (De Groot \& Steg, 2009, 2010). These findings also confirm the role of identity when examining the influence of values on environmental behavioural intentions, as observed in the experiments reported in Study 2.

Specifically, the results of Study 3 showed a significant positive effect of moral identity when "pure" ST values scores were used and also when only ST values scores were included, but not SE values scores. This supports the previous research that showed ST values had a more robust effect on environmental outcomes than SE values (e.g., Milfont, Sibley, \& Duckitt, 2010; Stern et al., 1998). Additionally, moral identity, or how people perceive themselves regarding what is morally expected, is closely related to morality which in turn has shown a strong relationship with ST values (Vauclair, 2010).

Most importantly, Study 3 reinforces that identity is an important variable to be considered when investigating the values-environmental behavioural intentions link. Indeed, having another layer of identity to explain the relationship between values and environmental behavioural intentions also adds to the empirical results presented in this thesis. The consistent finding across studies, that student identity, national identity and moral identity, influence the values-environmental behavioural intentions link reinforces the importance of studying identity to understand how values impact environmental outcomes. Furthermore, other moderators were not significant. Although self-efficacy, self-control and CFC have been shown to influence environmental 
engagement directly (e.g., Bamberg \& Moser, 2007; Milfont, Wilson, \& Diniz, 2012; Tabernero \& Hernandez, 2011), these variables did not moderate the valuesenvironmental behavioural intentions link in Study 3. This finding points especially to the relevance of moral identity for this topic of research.

Overall, the results of Study 3 converge with those found across the other two studies of this thesis in showing the importance of considering ST values as predictors of environmental outcomes as well as the role of identity in strengthening this relationship. Taken together, the results reveal a complex relationship between values, identity and environmental outcomes. Importantly, the three studies complement each other methodologically and have significant theoretical implications for the field. The next section draws on the theoretical framework forming the basis of this thesis (e.g., the Schwartz's values model) to interpret the results found and highlight the contributions of the thesis for theory and methodology.

\section{Contributions of this Thesis}

This thesis contributes to the values and environmental psychology literature in several ways. First, it adds to the discussion around the importance of values on environmental issues; specifically, it highlights the importance of the Schwartz's values model and the relationships between values in the circular model. Second, it adds to the implications of the VSC technique for value change and for understanding the dynamic character of values. Finally, it furthers the discussion on the role of identity for value change and shows how identity associated with values positively impacts environmental behavioural intentions.

\section{Theoretical Implications of the Current Thesis}

\subsection{Implications for the Schwartz's values model}

Values orientations are commonly regarded as crucial variables for understanding a number of constructs (Boer \& Fischer, 2013), including environmental outcomes (Hurst et al., 2013). Schwartz's theory has been one of the most well-known theories to study values (Schwartz, 1992), so it is not surprising that most of the extant literature linking values and environmental engagement is based on the Schwartz's values model. However, as shown in Study 1, there are also other values theories (e.g., 
Rokeach, 1973) in the psychological literature, as well as theoretical frameworks that have built on Schwartz's theory to create a specific model of values that relate to environmental engagement (Stern et al., 1998). The main dimensions that have consistently shown to be related to environmental matters is the ST vs. SE values dimensions. Different studies have attested to the positive versus negative relationships between these two sets of values and environmental outcomes (e.g., Schultz et al., 2005). These findings were replicated in the present thesis, and ST values were found to be the main positive predictors of environmental engagement.

However, Schultz et al. (2005) have shown that it is possible to find a positive relationship between SE values and environmental engagement, contrary to the typically expected negative relationship between these two constructs. Such a result was found in Study 1 of this thesis specifically with SE values positively predicting environmental concern and raises the question as to what would be the motivations or cognitive reasoning for SE-oriented people to care about the environment. As pointed out by Schultz et al., SE-oriented people might be motivated to engage in environmental protections given the negative impacts that environmental problems might have on their own welfare, compared to ST-oriented people who would focus on the environmental impacts on the welfare of others.

Nevertheless, ST values were the main values that predicted environmental outcomes in Study 1 and 3, and were also more prone to change in Study 2. It is not surprising that this dimension is related to environmental issues. As discussed in previous chapters, Schwartz (1992) defines ST values as characterised by a concern for the welfare and interest of others. Values such as benevolence and universalism are included in the ST values dimension and they specifically emphasise preserving and enhancing the welfare of the in-group (benevolence) and the understanding, appreciation, tolerance, and protection for the welfare of all people and for nature (universalism). In fact, Schwartz has recognised that environmental aspects are part of the motivations of ST oriented people and that people who are concerned about the welfare of others, by extension also care about the environment. On the other hand, SE values are characterised by one's pursuit of self-interests, involving values of power which are characterised by an emphasis on social status and prestige, control or dominance over people and resources; as well as values of achievement, which emphasises personal success through demonstrating competence according to social 
standards. Due to the self-centred character of the SE values, they are unlikely to be found in people who are more concerned about the environment then about themselves (Milfont, Sibley, \& Duckitt, 2010; Schultz et al., 2005).

One of the potential reasons why ST values were found in Study 2 to be more prone to change than SE values is that ST values incorporate an altruistic feature (Carlo, Eisenberg, Troyer, Switzer, \& Speer, 1991). Research on the topic has shown that altruistic people are more inclined to become more altruistic when confronted with their values (Diniz, 2009) because they feel they can do more or because they feel they are not doing enough (Carlo et al., 1991). Research has also shown that altruistic people tend to evaluate their own behaviour (Diniz, 2009; Mahoney \& Pechura, 1980), and so they are more inclined to change for the better, as they may feel that they can do even more to help others as they thrive to consistently become a better person (Carlo et al., 1991). In contrast, SE oriented people may be more resistant to change (Bain et al., 2012). Additionally, the three studies presented in this thesis, found that participants, especially in Study 2, had higher scores on ST values than they did on SE values. Similar results were observed in previous cross-cultural studies showing that individuals tend to place greater priorities on ST values compared to SE values (Schwartz, 1992). Theoretically, this suggests that individuals generally endorse more other-focused goals than self-focused goals, placing greater value on the welfare of others ahead of their own.

Given the strong link between ST values and environmental outcomes shown in the literature, the results reported in the present thesis highlight specific ways to increase environmental engagement. However, future research should examine more closely whether this greater emphasis on ST values overall is theoretically robust or merely a reflection of social desirability responding.

\subsection{Implications for the Use of the VSC Technique: The Underlying Mechanism of Value-Change and the Role of Identity}

Maio et al. (2009) proposed that according to the VSC technique and Schwartz's theory, values of the same dimension will increase if the reference group prioritises these values, whereas a decrease will be observed on values of the opposite dimension. Maio et al. also proposed that behaviour associated with certain values will also increase 
or decrease according to the values they are related to. Some of the findings in Study 2 replicate the previous results reported by Maio et al., while others contradicted their findings. Importantly, the contradiction between participants' own values and those of the reference group as confronted by the VSC technique follows the theoretical predictions of the impact of group pressure and social norms (Chernoff \& Davison, 2005). Based on this, the studies conducted in this thesis (Experiments 1 and 2), have offered an important insight from this research endeavour, which is the importance of identity on the mechanism of value change. Research has already investigated the possibility of changing values (Maio et al., 2009; Rokeach, 1973), but not much emphasis has been given to identity as a facilitator of value change. Researchers have also debated the durability of this change in values (e.g., Bardi \& Goodwin, 2011). Study 2 has shown that values can change, at least temporarily and, importantly, that the process of changing values is aided by identity (i.e., student and national identity).

The present research indicated that the VSC technique is effective, particularly for participants who highly identify with the reference group (university students or New Zealanders). The similar pattern of results (especially for ST values) in both experiments using different sample types and two different identity measures, gives a strong indication of the robustness of the VSC technique in changing values, as well as on the different types of identity that individuals can assume and their relevance for values and environmental intentions. Another important aspect is that most studies on the topic of value change have used the VSC technique but have not linked it to environmental outcomes (e.g., Maio et al., 2009). Results of Study 2 of this thesis have shown that the change on values enabled by the VSC technique predicts environmental behavioural intentions.

The effect of identity on values was again investigated in Study 3, with the possibility of exploring how another layer of identity might moderate the valuesenvironmental behavioural intentions link. It was found that moral identity did serve as a moderator of this relationship. Although previous studies have shown the direct and mediational effects of self-identity on environmental outcomes (e.g., Fielding et al., 2008; Whitmarsh \& O'Neill, 2010), as well as often demonstrated the direct effect of values on environmental engagement (e.g., Schultz \& Zelezny, 2009); no study has investigated what strengthens the link between values and environmental outcomes. The results in Chapter 5 showed that only moral identity (and not self-efficacy, self-control 
or CFC) moderated the relationship between values and environmental behavioural intentions, whereby moral identity enhanced the positive influence of ST values on environmental behavioural intentions. Perhaps, besides changing people's values and understanding identity as the underlining mechanism of value change, finding the right variables that should be reinforced to strengthen the link between values and environmental behavioural intentions is also essential to increase environmental engagement. Moral identity seems to be a crucial element in this process.

One important question is why moral identity moderates the values-environmental behavioural intentions link. One explanation for this is that people feel morally obliged to behave in a certain way, as people act on the basis of what is socially desirable and expected. People try to do what they value, and for people who think they have a strong moral identity it is crucial to "walk the talk". It is reasonable to think that people with strong moral identity may see that behaving in an environmentally friendly way is the right thing to do, and as a consequence, will behave in an environmentally friendly way.

In sum, previous studies that investigated the direct relationship between values and general prosocial attitudes using the VSC technique have not focused on identity (e.g., Schwartz \& Inbar-Saban, 1988). Studies have investigated the link between values and environmental engagement (e.g., Schultz \& Zelezny, 2009) and the link between identity and environmental engagement (e.g., Nigbur et al., 2010; Whitmarsh \& O’Neill, 2010), but no research has integrated these three variables. The present research does this and, to the author's knowledge, this thesis is the first to examine the effect of distinct identity types, including student, national and moral identity on the values-environmental behavioural intentions link. The results of the present research show that identity is an important variable that impacts on people's likelihood of changing values (Study 2), as well as moderates the relationship between values and environmental behavioural intentions (Study 3).

\section{Methodological Contributions of the Current Thesis}

A related contribution of the present study is to try to identify the boundary conditions for the influence of values on environmental outcomes, as discussed previously. Environmental outcomes are multi-determined and several constructs can explain in part why people behave towards the environment (Gifford, 2014). 
Understanding each piece of the puzzle is arduous but necessary and Studies 1, 2 and 3 add new pieces to this puzzle. The correlational design employed in Study 1 was important to identify and summarise the common findings in the literature, but it did not allow causal explanations. The experimental design used in Study 2 offered insights regarding causation, and in Study 3, the moderating role of identity was examined more systematically. More specifically, this thesis employed a multi-method approach to studying values and environmental outcomes. It used a meta-analysis (Lipsey \& Wilson, 2001) to identify the main values explaining environmental outcomes; an experimental design (Maio et al., 2009) to investigate value change and prediction of environmental behavioural intentions; and a survey to investigate moderational effects on the valuesenvironmental behavioural intentions link. The thesis contributes to these methodologies in a number of ways.

First, meta-analysis is a methodological approach which is explicitly concerned with integrating the findings of a large collection of analysis results (Glass, 1976; Lipsey \& Wilson, 2001). Because of this, it provides an overall and precise picture of the findings for a particular association between variables. Study 1 of this thesis used meta-analysis to assess the empirical research completed to date that has investigated values and environmental outcomes. Two previous meta-analyses have been conducted in the topic (Boer \& Fischer, 2013; Hurst et al., 2013), none however looking at the broader picture of all values theories and environmental outcomes. This study expanded the range of variables used in meta-analyses on this topic by extending beyond measures that only assess values using the Schwartz's values model, or a specific set of values such as materialistic values; and by assessing a broader range of environmental outcomes. This procedure was valuable in providing a broad idea of the associations between values and environmental outcomes. By focusing solely on the Schwartz's values measure or environmental attitudes, for example, like it has been done in the past, we would have missed many crucial values theories and crucial aspects of other environmental outcomes.

Second, Study 2 used an experimental approach to examine value change and environmental intentions. Few experimental studies have applied the VSC to influence value change (Maio et al, 2009; Schwartz \& Inbar-Saban, 1988). Additionally, no previous research has investigated the effect of this technique on changing values to predict environmental behavioural intentions. Furthermore, the VSC technique 
originally did not examine identity as the underlying mechanism of value change. The technique explicitly uses comparison with a reference group as a tool to make values salient. However, there is no indication that the original proposition of this technique and further developments took into consideration how strongly participants identify with the reference group that they are compared with. Study 2 results showed that two types of identity (student and national identity) influenced the effectiveness of this technique. We therefore recommend that researchers using this technique consider in future the importance of identity by establishing a meaningful reference group for the participants and the variables of interest.

Finally, Study 3 employed a moderation approach using a survey method to examine the relationships between values and environmental behavioural intentions, considering four moderators (i.e., moral identity, self-control, self-efficacy, and CFC). The findings of this study revealed that only moral identity enhanced the positive effect of ST values on environmental behavioural intentions. In the past, research has focused on the mediational (e.g., Dono et al., 2010; Fielding et al., 2008) effect of identity (selfidentity) in the relationship between values and environmental outcomes, or direct effects of identity (self-identity) on environmental engagement (e.g., Nigbur et al., 2010; Whitmarsh \& O’Neill, 2010). However, a strong claim for a moderation approach is that moral identity is a stable construct and, according to the literature, is closely related to ST values. Consequently, considering a moderation approach to study what strengthens the relationships between values and environmental outcomes is novel. Considering the positive outcomes found in Study 3, it is recommended that more research make use of moderational models to examine the moderating role of identity.

\section{Integrated Model of Values, Identity and Environmental Outcomes}

The results of the three studies presented in this thesis combine to offer a comprehensive portrayal of the relationship between values, identity and environmental engagement. This dissertation specifically investigated how values, more precisely ST and SE values, explain environmental outcomes. Figure 6.2 illustrates that values are the roots of the tree, underlying and nurturing environmental behavioural intentions, which are the leaves of the tree. As shown in Figure 6.2, the tree that has ST values as its roots is more nurtured, looks stronger and has abundant foliage (environmental 
behavioural intentions). However, the tree that has SE values as its roots is not well nourished and as a consequence produces fewer leaves of environmental behavioural intentions. A novel factor that was discovered in this thesis, is that identity (specifically student, national and moral identity) contributes to value change, and functions as an enhancer of the positive influence of ST values on environmental behavioural intentions. They are depicted in the diagram as the fertilisers of the tree. Finally, the results of Study 3 show that other potential fertilisers (i.e., self-control, self-efficacy and CFC) had no impact on enhancing the effect of ST values on environmental behavioural intentions, and so were not included in the figure. The results suggest that ST values should be targeted to promote environmental behavioural intentions and that student, national and moral identity should be considered when aiming to increase ST values or strengthen the relationships between these values and environmental outcomes. 


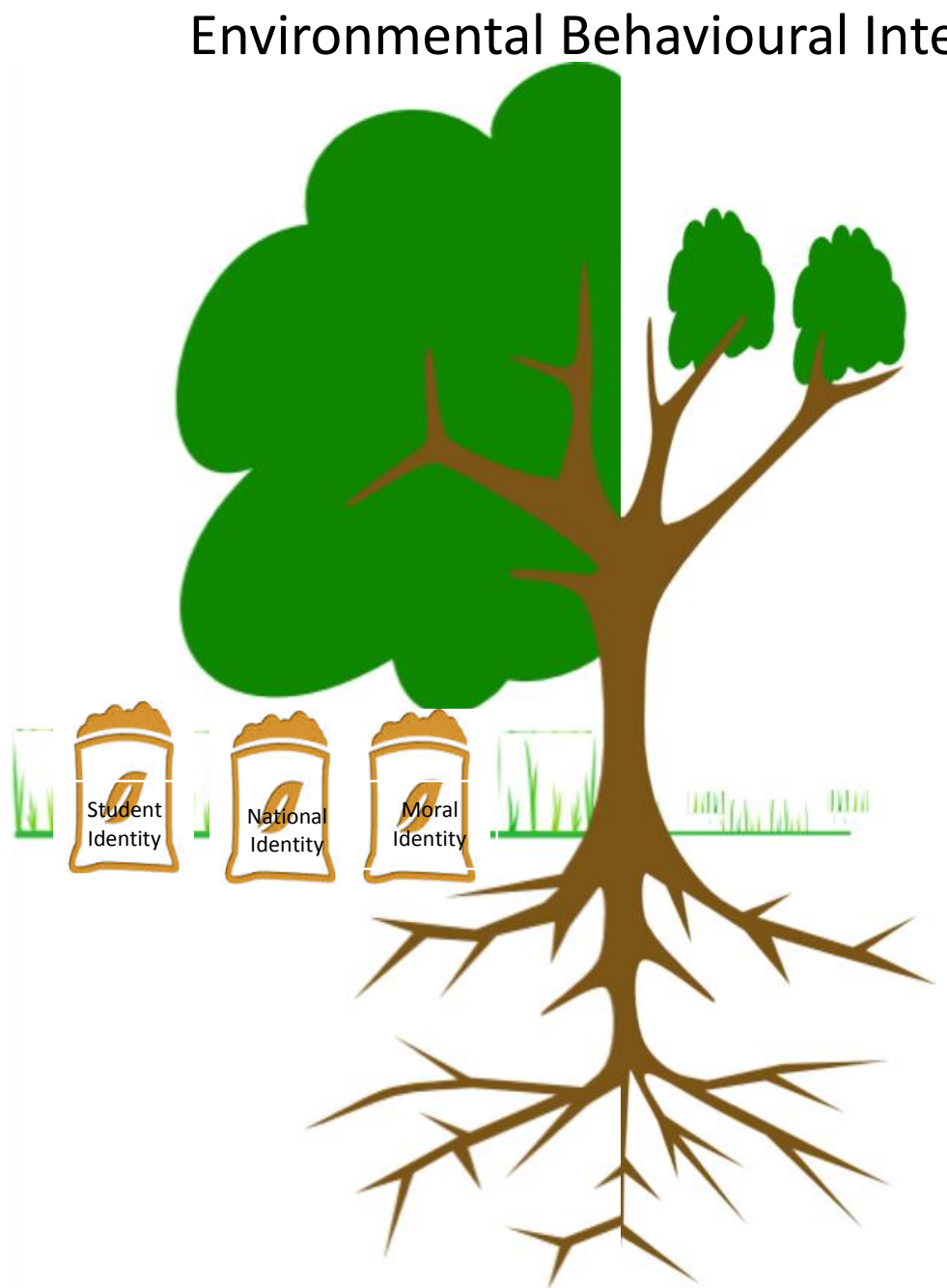

Self-transcendence values

Self-enhancement values

Figure 6.2. A comprehensive portrayal of values, identity and environmental behavioural intentions. 


\section{Practical Applications}

In addition to offering a strong body of theoretical implications and empirical effects, the findings from this thesis also have practical applications. Traditionally, interventions geared at promoting a more sustainable environment and environmental awareness have focused on distributing information in seminars, workshops, or classes. Although relevant, these practices have been shown to not be very effective (Schultz \& Kaiser, 2012). One reason for this is that people who are exposed to information, especially in a passive way (such as listening to a lecture or seminar), tend to not commit themselves with the content, or engage with it (Benware \& Deci, 1984).

In contrast with the education-based techniques, interventions based on social comparison and social influence have provided promising results (see Schultz et al., 2008). As the empirical findings of this thesis show, the VSC technique is an intervention based on social comparison designed to promote value change aimed to influence environmental behavioural intentions. First, the VSC technique asks participants to engage with the content in an active way (instead of a passive way). Next, they are asked to reflect on their own values and state what values are important to them. This activates a cognitive process of self-reflection about what they consider as important. When participants actively state what they believe, by writing and ordering the values according to their importance, they tend to engage more with the content at hand and, consequently, assimilate it more. In this way, the VSC technique has shown to be effective for changing values, and by changing values the VSC technique can be useful in influencing people's environmental behavioural intentions.

Knowing that we can change people's values is the first step in achieving the ultimate goal of making people more environmentally friendly. In practice, interventions could use social comparison in a positive way to lead people towards values that would promote environmental engagement. Importantly, the results also suggest that the VSC technique works better on particular values. Throughout this thesis, the results revealed the importance of ST values over SE values. Schools, universities and community centres can build a programme of intervention based on the VSC technique specifically aiming to enhance ST values, where participants would be asked to think through their values and notice them, while also making people reflect on the same values for a reference group that is meaningful to them, and on behaving in a 
more environmentally friendly way. Implementation of a programme along these lines may be extremely fruitful.

Furthermore, existing approaches based on environmental messages that aim to raise awareness or that use financial incentives and punishments have largely failed (Schultz, 2008; Schultz \& Kaiser, 2012). Campaigns that focus on awareness have highlighted the seriousness of environmental problems. This approach, as illustrated by Schultz (2008), emphasises the message that people are exhibiting undesirable behaviours which potentially undermine their efforts to promote conservation. In general, such campaigns or advertisements usually focus on the negative, on what has not been accomplished and what has been damaged. The findings in Chapter 4 suggest that the positive aspects of ST values (e.g., social justice and equality) should be fostered, instead of other values (e.g., SE values, Hurst et al., 2013) to promote environmental behavioural intentions, in line with the results from the meta-analysis in Chapter 3. Messages used in environmental campaigns would be more effective if they showed the collective benefits of acting in a more environmentally friendly way.

Additionally, advertisements could also focus on the positive features of what has been done so far to achieve a better world, avoiding a potential self-fulfilling prophecy for the target audience. According to the self-fulfilling prophecy, people tend to behave in the way that confirms social expectations (Merton, 1948). Consequently, if they are told that they are not protecting the environment, they may interpret that as society expecting them to act in that way, and they may continue to perform the negative behaviour to fulfil the general expectations portrayed in the media. Focusing on how people have the capacity to behave pro-environmentally may be more motivating then focusing on what has not been properly done to protect the environment. ST values are useful for that - they focus on the collective and are less inclined to prime values that are easily related to something negative, for example SE values.

Moreover, the results of this thesis suggest that practical interventions and advertisements will be more effective if they incorporate identity in their content. This research (specifically Study 2) has shown that making identity salient using group comparison can be an important contributor to promote value change which in turn can promote environmental engagement. Environmental campaigns should then consider the importance of choosing a meaningful reference group for social comparison, by making 
identity salient and subtly encouraging the target group to identify with the values promoted in the advertisements.

Additionally, in Study 3 it was evident that moral identity moderates the relationship between values and environmental behavioural intentions. Messages about values and environmental engagement are more likely to be retained if they include a component of moral identity too. A claim on moral identity would imply focusing on how people identify themselves with others in terms of what is good or bad, or right or wrong. For example, the messages transmitted to the public in campaigns that use advertisements to promote environmental practices, as well as intervention programmes, could focus on ST values (such as benevolence or world at peace) and include a group comparison (e.g., different nationalities) reflecting student, national and moral identity. Messages are likely to increase the likelihood of people acting in a more proenvironmental way if they focus on ST values change and promote the activation of social comparison mechanisms with a group of individuals that people highly identify with.

In general, the main practical contributions of the present thesis are twofold. First, the findings show that ST values are the main predictors of environmental outcomes, which can guide the creation of environmental campaigns. By linking proenvironmental actions with social justice and equality, such campaigns could be more successful. Second, the findings show the important role of identity. It is the combination of ST values and connectedness with a close group that motivates action. Again, environmental campaigns could focus on fostering identification with a particular community, and perhaps emphasise the benefits of pro-environmental actions to that community. Working on values is important to make people more environmentally friendly but identity is a crucial part in making this possible.

\section{Limitations}

Particular limitations for each study have already been discussed, but this section will review the main shortcomings of each study and the thesis as a whole. There are nine main limitations of the thesis. The first limitation is that, in Study 1, a large number of studies did not provide useful data for the meta-analysis. Other authors have reported facing the same difficulty in their meta-analyses (Hawcroft \& Milfont, 
2010; Hurst et al., 2013). Although the lack of information is an issue, especially for the studies that assessed environmental willingness and concern, in general, the studies that were included in the meta-analysis described in Study 1 reported adequate and reliable information, enabling the analysis. Additionally, the strength of the correlations between values and environmental outcomes were not very strong. However, the overall effect size found in Study 1 was similar in magnitude compared to other meta-analyses examining the same or similar variables (e.g., Boer \& Fischer, 2013).

The second limitation is that in the meta-analysis, it was difficult to break down the more specific details of the studies used to compose the data set. Theoretically, the grouping of the values dimensions made sense across different values theories and all theories were reduced to ST and SE values. Although this grouping was the most appropriate practically and logically according to the literature, it was also arbitrary and may not incorporate all the specific concepts of other theories. However, although the inability to check the specific concepts of all these theories is a limitation, it is an advantage to integrate the broad range of literature on one topic into one analysis. Nevertheless, in future research, it could be useful to examine how the individual value items (not only the value dimensions) relate to environmental outcomes. This would provide more detailed information about the associations between values and environmental engagement.

Thirdly, most of the studies that have used the Schwartz's theory to explain environmental issues have been located in English speaking countries, a trend evident in many areas of environmental psychology (Milfont \& Page, 2013). It is concerning that a majority of the data in the literature from which most conclusions regarding environmental issues are drawn reflect only western-developed, educated cultures. According to Boer and Fischer (2013), the Schwartz's dimensions affect general attitudes, including environmental attitudes, depending on the cultural context so it is important that future research strives to gather information from other parts of the world, not just Western cultures. Developing countries suffer as much as developed countries from environmental consequences, and have further to go in terms of educating people and creating sustainable habits that promote environmentally friendly acts. Consequently, gathering information regarding the drivers of citizens of developing countries' motivations for environmentally friendly behaviour can help to 
create a broader understanding of environmental engagement for different cultures and guide culturally targeted interventions.

A fourth limitation is that the two experiments described in Study 2 followed slightly different procedures and had different samples. While Experiment 1 was conducted at two different time points, with a computer-based questionnaire and a sample of university students, Experiment 2 was conducted at one time point, with a paper-based questionnaire and with a sample from the general population. Although these differences might be a limitation, it was also an advantage and the best design for using the reference group of New Zealanders to test the effect of identity on value change.

A fifth limitation concerns the generalization of the results. Although the present research offers important contributions to the literature and to practice, there needs to be caution in generalizing from the findings of some of the studies. Given that the samples of two of the empirical studies (Experiment 1 and Study 3) were composed mainly of undergraduate students, the findings may not be representative of the general population. This especially concerns the experimental results; a critique of experimental studies is that because they are conducted in an artificial environment (the lab), the generalization of the findings to the real world is limited. However, the experimental findings allow us to clarify a causal effect of values on environmental outcomes and Experiment 2 did examine the general population.

Limitation number six is the ceiling effect found for ST values. This limitation was pointed out in the specific discussion sections and concerns the fact that the majority of the participants scored high on ST values, making it difficult to have enough participants on the SE values dimension. As previously discussed, one reason for this is that SE values are considered undesirable (Sedikides et al., 2003). Consequently, participants might not genuinely have SE values or they may not have felt they could express them. The difficulties of assessing people who prioritise SE values may be a limitation of the results and could be investigated in future research.

Another limitation is that this thesis only focused on two values dimensions. In general, the results and discussion of the findings focused only on the ST and SE value dimensions. However, other dimensions have shown to be useful for specific environmental acts and could be explored in future research (Nordlund \& Garvill 2002; 
Shultz \& Zelezny, 1998). More specifically, the present thesis focused on one extreme of the dimension, ST values instead of SE values. Because SE values are often considered to be damaging to environmental engagement (e.g., Sedikides et al., 2003), research could investigate how to minimise its effect, and to identify what is the best strategy to assess people who are directed by those values.

Limitation number eight is regarding the consideration of only four moderators for the moderation study, which offered a limited, although not less relevant, conclusion. Specifically, it was shown that moral identity and not self-efficacy, selfcontrol or CFC, is an important contributor to the values-environmental behavioural intentions link. However, incorporating other variables into the model could offer a broader understanding of the relationship between values and environmental behavioural intentions. Research has shown that access to environmental alternatives (e.g., recycling system, carpooling, etc.) and public policies have a strong influence on environmental outcomes (Chen \& Tung, 2010). Furthermore, environmental outcomes are a multi-determined construct, and it would be a fruitful direction for future investigations to consider internal variables (e.g., identity) as well as external variables (e.g., public policies) to explain this construct.

A final limitation is that the environmental outcomes explored and assessed in this thesis were diverse, especially the ones assessed by the GEB. Research has shown that specific environmental outcomes or certain types of behaviours and attitudes are more influenced by certain types of values (Maio et al, 2009; Nordlund \& Garvill 2002; Schultz \& Zelezny, 1998; Schultz et al, 2005). Instead of a broad range of items assessing a large number of different environmental attitudes and behaviours, a particular set of environmentally related attitudes would give more information on how values influence certain types of environmental outcomes differently from others. For example, choosing to commute in public transport could be better related to openness to change than with ST values.

Despite these limitations, some consistent findings were obtained across three different methodologies (i.e., meta-analysis, experiments and survey research). The relationship between ST values and environmental outcomes was supported, and the findings also demonstrate the important role of identity (student, national and moral identity). Having some consistency of results across studies supports the reliability and validity of the findings throughout this thesis. 


\section{Future Research}

Three important questions remain. The first question is: Does the VSC technique work with other cultures? The current answer is, we do not know. The VSC technique has been shown to be effective in at least two English speaking countries: New Zealand (see Study 2 of this thesis) and the United Kingdom (Maio et al., 2009). However, this technique may work differently in other cultures. The VSC technique is based on social comparison and works with individuals who more closely identify with the target group. It may be the case that other cultural contexts emphasise social comparison differently and have different identity groups; future research could look into these cultural differences.

The second question is: Since changes in values were only observed in the ST values (versus SE values) dimension, would the technique be effective for other value dimensions too? This was not tested in the present thesis. Other dimensions of values such as openness to change have been shown to have an effect on certain types of environmental outcomes (Nordlund \& Westin, 2013). Although other values dimensions were not assessed in this study, they could also be affected by the VSC technique (Maio et al., 2009) and be affected differently by identity. It may be the case that people more open to change, for example, are more malleable in changing their values independently of how identified they feel with the group. In this particular example, although there is a group comparison, the focus is not on the group but on the actual activity that may represent innovation and bring specific pleasures to people prioritising openness to change.

Finally, the third question is: What can be done to improve the sense of identity for people with a low sense of identity? We know that identity is an important variable in the relationship between values and environmental issues, but how can identity be reinforced or strengthened? Previous research has indicated some directions that research could explore to reinforce identity (Constant \& Zimmermann, 2012) and future endeavours could look into this aspect in particular. However, rather than trying to increase identity (which also could have negatives effects), research could focus on identifying whether certain types of identity are more important to people and tapping into this relevant identity when trying to influence them to engage in environmentally sound behavioural intentions. 
In addition, because of time constraints, it was not possible to test the effect of value change long term. For this reason, the results only allow assumptions about the changes in values and the influence of values on environmental outcomes through identity using a short-term perspective. Longitudinal data would be valuable to allow an investigation of long-term changes and long-term influence of values on environmental behavioural intentions. Future research could focus on this aspect by looking at whether value change predicts environmental behavioural intentions across a longer time span. This is important because it would give valuable information on how to create habits that can be disseminated consistently among others and among generations (Harré, 2011).

Another suggestion for future studies is to test the effect of the VSC technique on actual environmental behaviour, not only intentions. Although previous studies have offered evidence of effects of the VSC technique on behaviour (Maio et al., 2009), this was not tested for environmental acts. Future research could explore if the VSC technique works in more concrete and less abstract domains of environmental acts.

In sum, the relationships between values, identity and environmental engagement is a topic with large potential for future research, and many unanswered questions still to be addressed. This thesis aimed to contribute to the clarification of some of these questions as well as raising new questions about the topic.

\section{Concluding Remarks}

Values motivate people's engagement in environmentally relevant acts. This thesis systemises the motivational underpinnings of environmental engagement and also explains the social mechanism of values-identity-environmental behavioural intentions. Most importantly, the predictions of values-environmental behavioural intentions links are facilitated by identity (student, national and moral identity). The two major motivational axes underpinning human values (ST and SE values) when related to environmental engagement are influenced by different degrees of association with a reference group or one's moral identity. These novel insights raise new research questions concerning the understanding of personal values and their implications for environmental engagement. This thesis suggests that future theories on motivational forces of environmentally relevant intentions should take account of group and moral 
identity. We cannot understand how values relate to environmental intentions unless we consider the strength of the association or identification within which individuals are interacting.

These theoretical and methodological advances should enhance integration of the field of environmental psychology. As environmental issues grow increasingly serious, it is important that research provides reliable and accurate information to increase practices that encourage people to live in a more environmentally friendly way. It is suggested that the results of the studies of this thesis will be valuable to future researchers in the topic.

Perhaps one of the most exciting contributions of this thesis is the body of knowledge that can be applied practically in our daily lives and in the real world. Thinking individually, knowing that our values are important predictors on how we make decisions on a daily basis regarding several domains including environmental engagement, we can recognise that questioning our life principles and challenging them can influence us to become more committed to environmental acts. Values can be changed, although this may not be easy and it takes significant exposure to value confrontation for this to happen. Encouraging us to confront our preferences can be one technique that may contribute to a long term change in our acts.

Noticing what people do around us and what type of values are fostered in our socio-networks and media can also be a source of explanation and questioning to understand our behavioural intentions. This insight can help individuals, especially people interested in acquiring knowledge regarding environmental challenges, and parents interested in raising a child in a more environmentally conscious context, to question their behaviour, along with the triggers leading them not to act in environmentally friendly ways, and the psychological reasoning behind it.

\section{My reflections on the thesis}

This thesis aimed to systematically assess the role of values in motivating individuals' pro-environmental engagement. Three empirical studies were conducted adopting different methodologies and analysis that built on each other. Amongst the many variables assessed and defined in this thesis two have special importance as they were the independent and dependent variables measured in all three empirical studies 
that were undertaken: values and environmental outcomes. Values are understood and defined as core principles guiding someone's life, in other words, values are motivational benchmarks that people use when making decisions. In this definition of values, it is important to note that values have a component of morality and are structured hierarchically according their importance. It is also important to note the controversial discussion around values stability and capability of change (further discussed below).

Environmental outcomes aggregate attitudes, behaviors, willingness and concern towards the environment. Each environmental outcome is distinct from each other and the complexity in defining and measuring them has caused a lot of confusion amongst researchers - this was evident in many studies that were incorporated in my metaanalysis. I have defined each of these specific concepts throughout the thesis (see chapter two) attending the need to precisely operationalize these variables. I have also decided to use the term environmental engagement to refer to all these four environmental outcomes assessed in my meta-analysis. The term serves to a practical purpose only - providing an umbrella term under which to reference environmental attitudes, behaviors, willingness (intentions) and concern. Furthermore, I do not consider attitudes, behaviors, willingness and concern as the same thing but instead I support the use of a term to refer to the group of different types of environmental outcomes.

In a world in which environmental issues have become a daily concern, the need to discover how to reinforce environmentally related human acts seems of high importance. The relevance of values to understand how people relate to the environment and the importance of changing values that can consequently foster environmental engagement are both well supported. This thesis suggests that social comparison can be used to effectively change people's values, and that identity constitutes the underlying mechanism to influence this value change. This thesis also showed that value change predicts environmental behavioural intentions. Through promoting value change we can reach the desired goal of making people more environmentally friendly, and contribute to a more sustainable planet.

In trying to experimentally manipulate values I faced two challenges: 1) promoting value change and 2) realizing that identity is not content free. Regarding the first challenge, the literature on values has emphasized the stability of values. However, 
I share the belief of some authors that the theoretical dynamism of the values dimensions can allow value change (even if it is in a short term; e.g., Maio et al., 2009). Furthermore, any change has to start from somewhere and making values salient can be the first step on the process a self-reflection and social comparison that triggers value change. Undoubtedly the long term effect of value change is highly desirable and should be pursued in future research, but the short term change is the logical first step towards achieving this.

Regarding the second challenge, the definition of identity depends on the type of identity one is referring to. For example, identity can be understood as characteristics belonging to one individual (personal identity) or shared by all members of a particular group (social identity). In Study 2, student and national identity was used and shown to have a strong effect on leading value-change. However, it is possible that identity may have been made (unintentionally) salient with the student sample. In Experiment 1, student identity was considered the most salient type of identity to be picked when using a sample of university students. The same rationale was used to justify focusing on national identity in the sample of New Zealanders in Experiment 2. However, in the experiment using university students, participants were measured together at the same time (differently from the general population). Although this wasn't shown to be a problem in previous studies it may have made students more likely to feel identified with the group they were tested with and may have influenced the results. To avoid that, I reaffirmed the steps that were taken during testing to minimize cross-participant influence, but I also suggest that future research could also perform multi-level analysis to identify any subtle group affects that can introduce bias.

Finally, Study 3 showed that moral identity is an important moderator of the value-environmental intentions link. In fact, moral identity enhanced the positive effect of self-transcendence values on environmental intentions. Moral identity is understood as a mental image or self-concept regarding what traits a person with strong morality holds. Although, the moderating effect moral identity shown in my final study is significant, it is also important to consider what type of moral identity has been assessed by the measure used in this thesis. The moral identity measure used in this thesis has a number of items that closely relate to self-transcendence values and may then express one type of moral identity more than others. The use of a broader measure tapping 
different aspects of moral identity (which also allow for specific aspects of samples) is suggested for future studies.

The three empirical studies of this thesis tell us a story on how values and environmental outcomes related to each other and can be changed and enhanced by identity (or identities). As any research endeavour, this thesis has had a number of sets backs as expected with any thesis of this complexity. However, those sets backs have helped to create a piece of work that's more critical and explorative. I shall finish this thesis on this note, proposing a new way of thinking about values-environment links and identity, and recognising the complexity and creative potential that sets backs in research (and in life) can have. 


\section{REFERENCES}

Achenreiner, G. B., (1997). Materialistic values and susceptibility to influence in children. Advances in Consumer Research, 24, 82-88.

Aitken, C., Chapman, R., \& McClure, J. (2011). Climate change, powerlessness and the commons dilemma. Global Environmental Change, 21, 752 -760.

Ajzen, I. (1985). From intentions to actions: A theory of planned behavior. In J. Kuhl \& J. Beckman (Eds.), Action-control: From cognition to behaviour (pp. 11-39). Heidelberg, Germany: Springer.

Ajzen, I. (1991). The theory of planned behaviour. Organizational Behaviour and Human Decision Processes, 50, 179-211.

Ajzen, I., \& Fishbein, M. (1980). Understanding attitudes and predicting social behaviour. Englewood Cliffs, NJ: Prentice-Hall.

Ajzen, I., \& Fishbein, M. (2005). The influence of attitudes on behavior. In D. Albarracín, B. T. Johnson, \& M. P. Zanna (Eds.), The handbook of attitudes (pp. 173221). Mahwah, NJ: Erlbaum.

Alexander, R. A., Scozzaro, M. J., \& Borodkin, L. J. (1989). Statistical and empirical examination of the chi-square test for homogeneity of correlations in metaanalysis. Psychological Bulletin, 106, 329-331.

Allport, G. W. (1960). The psychology of participation. Boston, MA: Beacon. Aquino, K., \& Reed, A., II. (2002). The self-importance of moral identity. Journal of Personality and Social Psychology, 83, 1423-1440.

Armitage, C. J., \& Conner, M. (2001). Efficacy of the theory of planned behaviour: A meta-analytic review. British Journal of Social Psychology, 40, 471-499.

Arnocky, S., Milfont, T. L., \& Nicol, J. (2014). Time perspective and sustainable behaviour: Evidence for the distinction between consideration of immediate and future consequences. Environment and Behavior, 46, 556-582.

Arnocky, S., Stroink, M., \& De Cicco, T. (2007). Self-construal predicts environmental concern, cooperation, and conservation. Journal of Environmental Psychology, 27, 255-264.

Axelrod, L. J. (1994). Balancing personal needs with environmental preservation: Identifying the values that guide decisions in ecological dilemmas. Journal of Social Issues, 50, 85-104. 
Bain, P. G., Hornsey, M. J., Bongiorno, R., \& Jeffries, C. (2012). Promoting pro-environmental action in climate change deniers. Nature Climate Change, 2, 600603.

Blodgett, J. G., Bakir, A., \& Rose, G. M. (2008). A test of the validity of Hofstede's cultural framework. Journal of Consumer Marketing, 25, 339-349.

Bamberg, S., \& Möser, G. (2007). Twenty years after Hines, Hungerford, and Tomera: A new meta-analysis of psycho-social determinants of pro-environmental behaviour. Journal of Environmental Psychology, 27, 14-25.

Bandura, A. (1986). Social foundations of thought and action: A social cognitive theory. Englewood Cliffs, NJ: Prentice-Hall.

Bandura, A. (1991). Social cognitive theory of self-regulation. Organizational Behavior and Human Decision Processes, 50, 248-287.

Bardi, A., Buchanan, K. E., Goodwin, R., Slabu, L., \& Robinson, M. (2014). Value stability and change during self-chosen life transitions: Self-selection versus socialization effects. Journal of Personality and Social Psychology, 106, 131-147.

Bardi, A., \& Goodwin, R. (2011). The dual route to value change: Individual processes and cultural moderators. Journal of Cross-Cultural Psychology, 42, 271-287.

Bargh, J. A., Gollwitzer, P. M., Lee-Chai, A., Barndollar, K., \& Trotschel, R. (2001). The automated will: Nonconscious activation and pursuit of behavioural goals. Journal of Personality and Social Psychology, 81, 1014-1027.

Barr, S. (2007). Factors influencing environmental attitudes and behaviors: A U.K. case study of household waste management. Environment and Behavior, 39, 435473.

Baumeister, R. F., \& Exline, J. J. (1999). Virtue, personality and social relations: Self-control as the moral muscle. Journal of Personality, 67, 1165-1194.

Baumeister, R. F., \& Tierney, J. (2011). Willpower: Rediscovering the greatest human strength. New York: Penguin.

Baumeister, R. F., Vohs, K. D., \& Tice, D. M. (2007). The strength model of self-control. Current Directions in Psychological Science, 16, 351-355.

Beaver, K. M., Hoffman, T., Shields, R. T., Vaughn, M. G., DeLisi, M., \& Wright, J. P. (2010). Gender differences in genetic and environmental influences on gambling: Results from a sample of twins from the National Longitudinal Study of Adolescent Health. Addiction, 105, 536-542. 
Beaver, K. M., Shutt, J. E., Boutwell, B. B., Ratchford, M., Roberts, K., \& Barnes, J. C. (2009). Genetic and environmental influences on levels of self-control and delinquent peer affiliation: Results from a longitudinal sample of adolescent twins. Criminal Justice and Behavior, 36, 41-60.

Beaver, K. M., Vaughn, M. G., \& DeLisi, M. (2013). Nonshared environmental effects on adulthood psychopathic personality traits: Results from a monozygotic twin difference scores analysis. Psychiatric Quarterly, 84, 381-393.

Becker, M., \& Félonneau, M. L. (2011). Pourquoi être pro-environnemental? Une approche socionormative des liens entre valeurs et pro-environnementalisme. Pratiques Psychologiques, 17, 237-250.

Beckmann, S. C. (2005). In the eye of the beholder: Danish consumer-citizens and sustainability. In K. G., Grunert, \& J. Thogersen (Eds.), Consumer, Policy and the Environment: A Tribute to Folke Olander (pp. 265-299). Berlin: Springer.

Benware, C. A. \& Deci, E. L. (1984). Quality of learning with an active versus passive motivational set. American Educational Research Journal, 21, 755-765. Berzonsky, M. D., \& Sullivan, C. (1992). Social-cognitive aspects of identity style: Need for cognition, experiential openness, and introspection. Journal of Adolescent Research, 7, 140-155.

Blodgett, J. G., Bakir, A., \& Rose, G. M. (2008). A test of the validity of Hofstede's cultural Framework. Journal of Consumer Marketing, 25/6, 339-349.

Boer, D., \& Fischer, R. (2013). How and when do personal values guide our attitudes and sociality? Explaining cross-cultural variability in attitude--value linkages. Psychological Bulletin, 139, 1113-1147. doi: http://dx.doi.org/10.1037/a0031347

Boisvert, D., Wright, J. P., Knopik, V., \& Vaske, J. (2012). Genetic and environmental overlap between low self-control and delinquency. Journal of Quantitative Criminology, 28, 477-507.

Bond, M. H., Leung, K., Au, A., Tong, K.-K., \& Chemonges-Nielson, Z. (2004). Combining Social Axioms with Values in Predicting Social Behaviours. European Journal of Personality, 18, 177-191.

Bonnes, M., Passafaro, P., \& Carrus, G. (2011). The ambivalence of attitudes toward urban green areas: Between proenvironmental worldviews and daily residential experience. Environment and Behavior, 43, 207-232. 
Braithwaite, V., Makkai, T., \& Pittelkow, Y. (1996). Inglehart's materialismpostmaterialism concept: Clarifying the dimensionality debate through Rokeach's model of social values. Journal of Applied Social Psychology, 26, 1536-1555.

Brennan, J., King, R., \& Lebeau, Y. (2004). The role of universities in the transformation of societies. An International Research Project. Association of Commonwealth Universities and Centre for Higher Education Research and Information. London, UK.

Bührs, T., \& Bartlett, R. V. (1993). Environmental policy in New Zealand: the politics of clean and green? Oxford, UK: Oxford University Press.

Caprara, G., Vecchione, M., \& Schwartz, S. H. (2009). Mediational role of values in linking personality traits to political orientation. Asian Journal of Social Psychology, $12,82-94$.

Carlo, G., Eisenberg, N., Troyer, D., Switzer, G., \& Speer, A. L. (1991). The altruistic personality: In what contexts is it apparent? Journal of Personality and Social Psychology 61, 450-458.

Carlson, B. R., \& Edwards, W. H. (1987). Human values and cocaine use. Journal of Drug Education, 17, 183-195.

Chen, M. F., \& Tung, P. J. (2010). The moderating effect of perceived lack of facilities on consumers' recycling intentions. Environment and Behavior, 42, 824-844. Chen, X.-P., Wasti, S. A., \& Triandis, H. C. (2007). When does group norm or group identity predict cooperation in a public goods dilemma? The moderating effects of idiocentrism and allocentrism. International Journal of Intercultural Relations, 31, 259276.

Chernoff, R. A., \& Davison, G. C. (2005). An evaluation of a brief HIV/AIDS prevention intervention for college students using normative feedback and goal setting. AIDS Education and Prevention, 17, 91-104.

Cho, Y.-N., Thyroff, A., Rapert, M. I., Park, S.-Y., \& Lee, H. J. (2013). To be or not to be green: Exploring individualism and collectivism as antecedents of environmental behavior. Journal of Business Research, 66, 1052-1059.

Chun, R. (2009). Ethical values and environmentalism in China: Comparing employees from state-owned and private firms. Journal of Business Ethics, 84, 341-348.

Clark, T. (2011). Assessing the accuracy of manipulation checks: Follow-up. Undergraduate Honors Thesis Series, East Tennessee State University. 
Cohen, J. (1988). Statistical power analysis for the behavioural sciences. Hillsdale, NJ: Erlbaum.

Conn, V. S. \& Rantz, M. J. (2003). Focus on research methods: Managing primary study quality in meta-analyses. Research in Nursing \& Health, 26, 322-333. Constant, A. F. \& Zimmermann, K. F. (2013). Immigrants, ethnic identities and the nation-state. In F. Constant \& K. F. Zimmermann (Eds.), International handbook on the economics of migration, (pp. 259-275). Cheltenham, UK: Edward Elgar.

Corral-Verdugo, V., Fraijo-Sing, B., \& Pinheiro, J. Q. (2006). Sustainable behavior and time perspective: Present, past, and future orientations and their relationship with water conservation behavior. Revista Interamericana de Psicología, 40, 139-147.

Cozby, P. C. (2009). Methods in behavioral research. Boston, MA: McGrawHill Higher Education.

Crompton, T. (2010). Common cause - The case for working with our cultural values. Godalming, UK: WWF-UK.

Datler, G., Jagodzinski, W., \& Schmidt, P. (2013). Two theories on the test bench: Internal and external validity of the theories of Ronald Inglehart and Shalom Schwartz. Social Science Research, 42, 906-925.

Davenport, C. \& Parker, A. (2014, October 21). Environmental is grabbing big role in ads for campaigns. The New York Times. Retrieved from: http://www.nytimes.com/2014/10/22/us/politics/environmental-issues-become-a-forcein-political-advertising.html? $\mathrm{r}=0$

Davis, T., \& Francis, J. E. (2014). The young consumer-citizen: Nationhood and environmentalism in children's identity narratives. Marketing Theory, 14, 417-429.

De Groot, J., \& Steg, L. (2007). General beliefs and the theory of planned behavior: The role of environmental concerns in the TPB. Journal of Applied Social Psychology, 37, 1817-1836.

De Groot, J. I. M., \& Steg, L. (2008). Value orientations to explain beliefs related to environmental significant behavior: How to measure egoistic, altruistic, and biospheric value orientations. Environment and Behavior, 40, 330-354.

De Groot, J. I. M., \& Steg, L. (2009). Morality and prosocial behavior: the role of awareness, responsibility and norms in the norm activation model. Journal of Social Psychology, 149. doi: 425-449, DOI: 10.3200/SOCP.149.4.425-449 
De Groot, J. I. M., \& Steg, L. (2010). Relationships between value orientations, self-determined motivational types and pro-environmental behavioural intentions. Journal of Environmental Psychology, 30, 368-378.

Devine, P. G., Plant, E. A., \& Buswell, B. N. (2000). Breaking the prejudice habit: Progress and obstacles. Mahwah, NJ: Erlbaum.

Devine-Wright, P. (2013). Explaining "NIMBY" objections to a power line: The role of personal, place attachment and project-related factors. Environment and Behavior, 45, 761-781.

Diamantopoulos, A., Schlegelmilch, B. B., Sinkovics, R. R., \& Bohlen, G. M. (2003). Can socio-demographics still play a role in profiling green consumers? A review of the evidence and an empirical investigation. Journal of Business Research, $56,465-480$.

Diniz, P. K. C. (2009). Values and emotional correlates of altruism. Unpublished Masters thesis. Federal University of Paraíba, João Pessoa, Brazil. Diniz, P. K. C., Fischer, R., Milfont, T. L., \& McClure, J. (2012, September). Do values explain environmental engagement? A meta-analytical review of cross-cultural data. Paper presented at 21st International Organization of Cross-cultural Psychology Conference, Stellenbosch, South Africa.

Diniz, P. K. C., Milfont, T. L., Fischer, R., \& McClure, J. (2013a, July). Using a meta-analytical approach to assess the relationship between values and environmental engagement. In H. Günther (Chair), O que influencia o que? Exemplos da relação entre comportamento humano e meio-ambiente. Symposium at the $34^{\text {th }}$ Interamerican Congress of Psychology, Brasilia, Brazil.

Diniz, P. K. C., Milfont, T. L., Fischer, R., \& McClure, J. (2013b, June). Underlining motivational mechanisms of values and national identity: A study on promoting environmental behavioural intentions. Paper presented at the regional conference of the International Association of Cross-Cultural Psychology, Los Angeles, USA.

Diniz, P. K. C., Fischer, R., Milfont, T. L. \& McClure, J. (2013c, July). The underlining mechanism of value change: A study on identity and environmental engagement. Paper presented at the 13th European Congress of Psychology, Stockholm, Sweden.

Diniz, P. K. C., Milfont, T. L., \& McClure, J. (2012, September). Psychometric parameters of a modified version of the General Ecological Behavioural (GEB).Poster 
presentation at the 22nd International Association of People-Environment Studies Conference, Glasgow, Scotland.

Diniz, P. K. C., Milfont, T. L., McClure, J., \& Fischer, R. (2012, September). Experimentally manipulating values to motivate pro-environmental behavioural intentions. Paper presented at 22nd International Association of People-Environment Studies Conference, Glasgow, Scotland.

Djupe, P. A., \& Calfano, B. R. (2013). Religious value priming, threat, and political tolerance. Political Research Quarterly, 66, 768-780.

Dobewall, H., \& Rudnev, M. (2013). Common and unique features of Schwartz's and Inglehart's value theories at the country and individual levels. CrossCultural Research: The Journal of Comparative Social Science, 48, 45-77.

Dono, J., Webb, J., \& Richardson, B. (2010). The relationship between environmental activism, pro-environmental behaviour and social identity. Journal of Environmental Psychology, 30, 178-186.

Döring, A. K., Schwartz, S. H., Cieciuch, J., Groenen, P. J. F., Glatzel, V., Harasimczuk, J., ... Bilsky, W. (2015). Cross-cultural evidence of value structures and priorities in childhood. British Journal of Psychology, 106, 675-699.

Dunlap, R., \& Jones, R. E. (2002). Environmental concern: Conceptual and measurement issues. Westport, CT: Greenwood.

Dunlap, R. E., Grieneeks, J. K., \& Rokeach, M. (1983). Human values and proenvironmental behavior. Boulder, CO: Westview.

Dunlap, R. E., \& Mertig, A. G. (1995). Global concern for the environment: Is affluence a prerequisite? The Journal of Social Issues, 51, 121.

Dunlap, R., \& Van Liere, K. (1978). The new environmental paradigm. The Journal of Environmental Education, 9, 10-19.

Dunlap, R. E., Van Liere, K. D., Mertig, A. G., \& Jones, R. E. (2000). Measuring endorsement of the new ecological paradigm: A revised NEP scale. The Journal of Social Issues, 56, 425-442.

Eagly, A. H., \& Chaiken, S. (1993). The psychology of attitudes. Fort Worth, TX: Harcourt, Brace, Jovanovich.

Evans, L., Maio, G. R., Corner, A., Hodgetts, C. J., Ahmed, S., \& Hahn, U. (2013). Self-interest and pro-environmental behaviour. Nature Climate Change, 3, $122-$ 125. 
Farley, E (1990). Good and Evil: Interpreting a human condition. Fortress Press: Vanderbilt University.

Fanelli, D. (2012). Negative results are disappearing from most disciplines and countries. Scientometrics, 90, 891-904.

Fazio, R. H., \& Towles-Schwen, T. (1999). The MODE model of attitude behaviour process. New York, NY: Guilford Press.

Fazio, R. H., \& Zanna, M. P. (1978). On the predictive validity of attitudes: The roles of direct experience and confidence. Journal of Personality, 46, 228-243. doi: http://dx.doi.org/10.1111/j.1467-6494.1978.tb00177.x

Feather, N. T. (1996). Extending the search for order in social motivation: Comment. Psychological Inquiry, 7, 223-229. doi: ttp://dx.doi.org/10.1207/s15327965pli0703_4

Feather, N. T. (2002). Reactions to supporters and opponents of uranium mining in relation to status, attitude similarity, and right-wing authoritarianism. Journal of Applied Social Psychology, 32, 1464-1487.

Festinger, L. (1957). A theory of cognitive dissonance. Stanford, CA: Stanford University Press.

Feygina, I., Jost, J. T., \& Goldsmith , R. E. (2010). System justification, the denial of global warming, and the possibility of "System-Sanctioned Change". Personality and Social Psychology Bulletin, 36, 326-338.

Feldman, D. C. (1984). The development and enforcement of group norms. Academy of Management Review, 9, 47-53.

Fielding, K. S., McDonald, R., \& Louis, W. R. (2008). Theory of planned behaviour, identity and intentions to engage in environmental activism. Journal of Environmental Psychology, 28, 318-326.

Fischer, R. (2012). Value isomorphism in the European Social Survey: Exploration of meaning shifts in values across levels. Journal of Cross-Cultural Psychology, 43, 883-898.

Fischer, R., \& Poortinga, Y. H. (2012). Are cultural values the same as the values of individuals? An examination of similarities in personal, social and cultural value structures. International Journal of Cross Cultural Management, 12, 157-170.

Fischer, R., Vauclair, C. M., Fontaine, J. R. J., \& Schwartz, S. H. (2010). Are individual-level and country-level value structures different? Testing Hofstede's legacy with the Schwartz Value Survey. Journal of Cross-Cultural Psychology, 41, 135-151. 
Fishbein, M. (2008). A reasoned action approach to health promotion. Medical Decision Making, 28, 834-844.

Fishbein, M., \& Ajzen, I. (2005). Theory-based behavior change interventions: Comments on Hobbis and Sutton. Journal of Health Psychology, 10, 27-31.

Fransson, N., \& Gärling, T. (1999). Environmental concern: Conceptual definitions, measurement methods, and research findings. Journal of Environmental Psychology, 19, 369-382.

Fritsche, I., Cohrs, J. C., Kessler, T., \& Bauer, J. (2012). Global warming is breeding social conflict: The subtle impact of climate change threat on authoritarian tendencies. Journal of Environmental Psychology, 32, 1-10.

Fritzsche, D. J., \& Oz, E. (2007). Personal values' influence on the ethical dimension of decision making. Journal of Business Ethics, 75, 335-343.

Fukukawa, K., Shafer, W. E., \& Lee, G. M. (2007). Values and attitudes toward social and environmental accountability: A study of MBA students. Journal of Business Ethics, 71, 381-394.

Gadermann, A. M., Guhn, M. \& Zumbo, B. D. (2012). Estimating ordinal reliability for Likert-type and ordinal item response data: A conceptual, empirical, and practical guide. Practical Assessment, Research and Evaluation, 17, 3-13.

Gardner, G. T., \& Stern, P. C. (2002). Environmental problems and human behavior. Boston, MA: Pearson Custom Publishing.

Gifford, R. (2007). Environmental Psychology: Principles and Practice. Colville, WA: Optimal Books.

Gifford, R. (2008). Psychology's essential role in alleviating the impacts of climate change. Canadian Psychology, 49, 273-280.

Gifford, R. (2014). Environmental psychology matters. Annual Review of Psychology, 65, 541-580.

Glass, G. V. (1976). Primary, secondary, and meta-analysis of research. Educational Research, 5, 3-8.

Godin, G., \& Kok, G. (1996). The theory of planned behavior: A review of its applications to health-related behaviors. American Journal of Health Promotion, 11, $87-$ 98.

Gökşen, F., Adaman, F., \& Zengınobuz, E. Ü. (2002). On environmental concern, willingness to pay, and postmaterialist values: Evidence from Istanbul. Environment and Behavior, 34, 616-633. 
Gollwitzer, P. M. (1999). Implementation intentions: Strong effects of simple plans. American Psychologist, 54, 493-503.

Gooch, G. D. (1995). Environmental beliefs and attitudes in Sweden and the Baltic States. Environment and Behavior, 27, 513-539.

Gosling, S. D., Rentfrow, P. J., \& Swann, W. B., Jr. (2003). A very brief measure of the Big-Five personality domains. Journal of Research in Personality, 37, 504-528.

Grønhøj, A., \& Thøgersen, J. (2012). Action speaks louder than words: The effect of personal attitudes and family norms on adolescents' pro-environmental behaviour. Journal of Economic Psychology, 33, 292-302.

Grunert, S., \& Juhl, H. J. (1995). Values, environmental attitudes, and buying of organic foods. Journal of Economic Psychology, 16, 39-62.

Guagnano, G. A., \& Markee, N. (1995). Regional differences in the sociodemographic determinants of environmental concern. Population and Environment: A Journal of Interdisciplinary Studies, 17, 135-149.

Haller, M., \& Hadler, M. (2008). Dispositions to act in favor of the environment: Fatalism and readiness to make sacrifices in a cross-national perspective. Sociological Forum, 23, 281-311.

Hansla, A., Gamble, A., Juliusson, A., \& Garling, T. (2008). The relationships between awareness of consequences, environmental concern, and value orientations. Journal of Environmental Psychology, 28, 1-9.

Hansla, A., Gärling, T., \& Biel, A. (2013). Attitude toward environmental policy measures related to value orientation. Journal of Applied Social Psychology, 43, 582590.

Harding, S., \& Phillips, D. (1986). Contrasting values in Western Europe. Unity, diversity and change. London: Macmillan.

Hardy, S. A., Walker, L. J., Olsen, J. A., Woodbury, R. D., \& Hickman, J. R. (2014). Moral identity as moral ideal self: Links to adolescent outcomes. Developmental Psychology, 50, 45-57.

Harré, N. F. (2011). Psychology for a better world: Strategies to inspire sustainability. Auckland: Department of Psychology, University of Auckland. Retrieved from: http://hdl.handle.net/2292/14172.

Hart, D. (2005). Adding identity to the moral domain. Human Development, 48, 257-261. 
Hausbeck, K. W., Milbrath, L. W., \& Enright, S. M. (1992). Environmental knowledge, awareness and concern among 11th-grade students: New York State. The Journal of Environmental Education, 24, 27-34.

Hausenblas, H. A., Carron, A. V., \& Mack, D. E. (1997). Application of the theories of reasoned action and planned behavior to exercise behavior: A meta-analysis. Journal of Sport \& Exercise Psychology, 19, 36-51.

Hawcroft, L. J., \& Milfont, T. L. (2010). The use (and abuse) of the new Environmental Paradigm Scale over the last 30 years: A meta-analysis. Journal of Environmental Psychology, 30, 143-158.

Heath, R. L., \& Fogel, D. S. (1978). Terminal and instrumental? An inquiry into Rokeach's value survey. Psychological Reports, 42, 1147-1154.

Heath, Y., \& Gifford, R. (2006). Free-market ideology and environmental degradation: The case of belief in global climate change. Environment and Behavior, $38,48-71$.

Hedges, L., \& Olkin, I. (1985). Statistical methods for meta-analysis. Orlando, FL: Academic Press.

Hendrickx, L., Poortinga, W., \& van der Kooij, R. (2001). Temporal factors in resource dilemmas. Acta Psychologica, 108, 137-154.

Henrich, J., Heine, S. J., \& Norenzayan, A. (2010). The weirdest people in the world? Behavioral and Brain Sciences, 33, 1-75.

Hines, J. M., Hungerford, H. R., \& Tomera, A. N. (1986/1987). Analysis and synthesis of research on responsible environmental behavior: A meta-analysis. The Journal of Environmental Education, 18, 1-8.

Hirsh, J. B. (2010). Personality and environmental concern. Journal of Environmental Psychology, 30, 245-248.

Hitlin, S. (2003). Values as the core of personal identity: Drawing links between two theories of self. Social Psychology Quarterly, 66, 118-137.

Hofstede, G. (1983). Dimensions of national cultures. In R. Rath, H. S. Asthana, D. Sinha \& J. B. H. Sinha (Eds.), Diversity and unity in cross-cultural psychology (pp. 335-355). Lisse, Netherlands: Swets and Zeitlinger.

Hofstede, G. (1991). Cultures and organizations. London: McGraw-Hill Book Company.

Hofstede, G. (2001). Culture's consequences. Thousand Oaks, CA: Sage. 
Hofstede, G., \& Bond, M. H. (1984). Hofstede's culture dimensions: An independent validation using Rokeach's Value Survey. Journal of Cross-Cultural Psychology, 15, 417-433.

Homburg, A., \& Stolberg, A. (2006). Explaining pro-environmental behavior with a cognitive theory of stress. Journal of Environmental Psychology, 26, 1-14.

Homer, P. M., \& Kahle, L. R. (1988). A structural equation test of the valueattitude-behavior hierarchy. Journal of Personality and Social Psychology, 54, 638-646.

Hurst, M., Dittmar, H., Bond, R., \& Kasser, T. (2013). The relationship between materialistic values and environmental attitudes and behaviors: A meta-analysis. Journal of Environmental Psychology, 36, 257-269.

Inglehart, R. (1981). Post-materialism in an environment of insecurity. American Political Science Review, 79, 97-116.

Inglehart, R. (1990). Culture shift in advanced industrialist society. Princeton, NJ: Princeton University Press.

Inglehart, R. (1995). Public support for the environmental protection: Objective problems and subjective values in 43 societies. Political Science \& Politics, 28, 57-72.

Inglehart, R. (1997). Modernization and postmodernization. Cultural, economic and political change in 43 societies. Princeton, NJ: Princeton University Press. Inglehart, R. (2008) Changing values among Western publics from 1970 to 2006. West European Politics, 31, 130-146

Inglehart, R., \& Baker, W. E. (2000). Modernization, cultural change, and the persistence of traditional values. American Sociological Review, 65, 19-51.

Inoue, Y. \& Alfaro-Barrantes, P. (2015). Pro-environmental behavior in the workplace: A review of empirical studies and directions for future research. Business and Society Review, 120, 137-160.

Joireman, J., Balliet, D., Sprott, D., Spangenberg, E., \& Schultz, J. (2008). Consideration of future consequences, ego-depletion, and self-control: Support for distinguishing between CFC-Immediate and CFC-Future sub-scales. Personality and Individual Differences, 45, 15-21.

Joireman, J. A., Van Lange, P .A. M., \& Van Vugt, M. (2004). Who cares about the environmental impact of cars? Those with an eye toward the future. Environment and Behavior, 36, 187-206.

Jose, P.E. (2013). ModGraph-I: A programme to compute cell means for the graphical display of moderational analyses: The internet version, Version 3.0. Victoria 
University of Wellington, Wellington, New Zealand. Retrieved from http://pavlov.psyc.vuw.ac.nz/paul-jose/modgraph/

Kaiser, F. G., \& Byrka, K. (2011). Environmentalism as a trait: Gauging people's prosocial personality in terms of environmental engagement. International Journal of Psychology, 46, 71-79.

Kaiser, F. G., Byrka, K., \& Hartig, T. (2010). Reviving Campbell's paradigm for attitude research. Personality and Social Psychology Review, 14, 351-367.

Kaiser, F. G., Ranney, M., Hartig, T., \& Bowler, P. A. (1999). Ecological behavior, environmental attitude, and feelings of responsibility for the environment. European Psychologist, 4, 59-74. doi: http://dx.doi.org/10.1027//1016-9040.4.2.59 Kaiser, F. G., Wölfing, S., \& Fuhrer, U. (1999). Environmental attitude and ecological behaviour. Journal of Environmental Psychology, 19, 1-19. doi:

http://dx.doi.org/10.1006/jevp.1998.0107

Kaiser, F. G., \& Wilson, M. (2004) Goal-directed conservation behavior: the specific composition of a general performance. Personality and Individual Differences, $36,1531-1544$.

Karp, D. G. (1996). Values and their effect on pro-environmental behavior. Environment and Behavior, 28, 111-133.

Kelly, M., Tovey, H., \& Faughnan, P. (2007). Environmental attitudes, values and behaviour in Ireland. Dublin, Ireland: Environmental Protection Agency.

Kilby, R. W. (1993). The study of human values. Lanham, UK: University Press of America.

Kilmann, R. H. (1981). Toward a unique/useful concept of values for interpersonal behavior: A critical review of the literature on value. Psychological Reports, 48, 939-959. doi: http://dx.doi.org/10.2466/pr0.1981.48.3.939

Kim, Y. H. (1994). A survey on environmental attitudes and behavior among city residents in Korea. Korean Journal of Social Psychology, 8, 85-102.

Kortenkamp, K. V., \& Moore, C. F. (2006). Time, uncertainty, and individual differences in decisions to cooperate in resource dilemmas. Personality and Social Psychology Bulletin, 32, 603-615.

Krosnick, J. A., \& Petty, R. E. (1995). Attitude strength: Antecedents and consequences. Hillsdale, NJ: Erlbaum. 
Lalwani, A. K., Shavitt, S., \& Johnson, T. (2006). What is the relation between cultural orientation and socially desirable responding? Journal of Personality and Social Psychology, 90, 165-178.

Last week tonight with John Oliver (2014, May 11). Climate change debate. Retrieved from https://www.youtube.com/watch?v=cjuGCJJUGsg\#t=12

Lawrence, J., Cornforth, A., \& Barrett, P. (2011). Climate futures - Pathways for society. Victoria University of Wellington, New Zealand Climate Change Research Institute.

Leary, M. R., Bednarski, R., Hammon, D., \& Duncan, T. (1997). Blowhards, snobs, and narcissists: Interpersonal reactions to excessive egotism. In R. M. Kowalski (Ed.), Aversive interpersonal behaviors (pp. 111-131). New York: Plenum.

Leonard-Barton, D. (1981). Voluntary simplicity lifestyles and energy conservation. Journal of Consumer Research, 8, 243-252.

Leonenko, G., Los, S. O., \& North, P. R. J. (2013). Statistical distances and their applications to biophysical parameter estimation: Information measures, M-estimates, and minimum contrast methods. Remote Sensing, 5, 1355-1388.

Levine, D. S. (1997). Sex and human values. PsycCRITIQUES, 42, 1118-1119. Lévy-Leboyer, C., Bonnes, M., Chase, J., Ferreira-Marques, J., \& Pawlik, K. (1996). Determinants of pro-environmental behaviors: A five-countries comparison. European Psychologist, 1, 123-129.

Lipsey, M. W., \& Wilson, D. B. (2001). Practical meta-analysis. Thousand Oaks, CA: Sage.

Luhtanen, R., \& Crocker, J. (1992). A collective self-esteem scale: Selfevaluation of one's social identity. Personality and Social Psychology Bulletin, 18, 302318.

MacCallum, R. C., Zhang, S., Preacher, K. J. \& Rucker, D. D. (2002). On the practice of dichotomization of quantitative variables. Psychological Methods, 7, 19-40

Macrae, C. N., \& Johnston, L. (1998). Help, I need somebody: Automatic action and inaction. Social Cognition, 16, 400-417.

Mahoney, J. \& Pechura, C. M. (1980). Values and volunteers: axiology of altruism in a crisis center. Psychological Report, 47, 1007-1012

Maio, G. R., \& Olson, J. M. (1995). Relations between values, attitudes, and behavioral intentions: The moderating role of attitude function. Journal of Experimental Social Psychology, 31, 266-285. 
Maio, G. R. \& Olson, J. M. (1998). Values as truisms: Evidence and implications. Journal of Personality and Social Psychology, 74, 294-311.

Maio, G. R., Pakizeh, A., Cheung, W.-Y., \& Rees, K. J. (2009). Changing, priming, and acting on values: Effects via motivational relations in a circular model. Journal of Personality and Social Psychology, 97, 699-715.

Maloney, M. P., \& Ward, M. P. (1973). Ecology: Let's hear from the people: An objective scale for the measurement of ecological attitudes and knowledge. American Psychologist, 28, 583-586.

Maloney, M. P., Ward, M. P., Braucht, G. N. (1975). A revised scale for the measurement of ecological attitudes and knowledge. American Psychologist, 30, 787790.

Manning, M., Reisinger, A., \& Wratt, D. (2009). A summary of the assessment process of the intergovernmental panel on climate change - An invited presentation to the New Zealand Emissions Trading Scheme Review Committee. Wellington, NZ: New Zealand Climate Change Research Institute, Victoria University of Wellington.

Marcia, J. E. (1966). Development and validation of ego identity status. Journal of Personality and Social Psychology, 5, 551-558.

Markus, H. R., \& Kitayama, S. (1991). Culture and the self: Implications for cognition, emotion, and motivation. Psychological Review, 98, 224-253.

Masters, J.C., Carlson, C.R., \& Rahe, D. (1985). Children's affective, behavioral, and cognitive responses to social comparison. Journal of Experimental Social Psychology, 21, 407-420.

Matsuba, M. K., Pratt, M. W., Norris, J. E., Mohle, E., Alisat, S., \& McAdams, D. P. (2012). Environmentalism as a context for expressing identity and generativity: Patterns among activists and uninvolved youth and midlife adults. Journal of Personality, 80, 1091-1115.

Memon, P. A. (1993). Keeping New Zealand green: Recent environmental reforms. Dunedin, New Zealand: Otago University Press.

Merton, R. K. (1948). The self-fulfilling prophecy. The Antioch Review, 8(2), 193-210.

Mescheloff-Faran, Y. (2010). The effect of values priming on the willingness to drive recklessly among young drivers. Unpublished Master's thesis, Bar-Ilan University, Ramat Gan, Israel. 
Milfont, T. L. (2007). Psychology of environmental attitudes: A cross-cultural study of their content and structure. Unpublished doctoral dissertation, University of Auckland, Auckland, New Zealand.

Milfont, T. L. (2009a). The effects of social desirability on self-reported environmental attitudes and ecological behaviour. Environmentalist, 29, 263-269.

Milfont, T. L. (2009b). A functional approach to the study of environmental attitudes. Psyecology, 10, 235-252.

Milfont, T. L. (2012). Cultural differences in environmental engagement. In S. Clayton (Ed.). The Oxford Handbook of Environmental and Conservation Psychology (pp. 181-202). Oxford, UK: Oxford University Press.

Milfont, T. L., \& Duckitt, J. (2004). The structure of environmental attitudes: A first- and second-order confirmatory factor analysis. Journal of Environmental Psychology, 24, 289-303.

Milfont, T. L., Duckitt, J., \& Wagner, C. (2010). A cross-cultural test of the value-attitude-behavior hierarchy. Journal of Applied Social Psychology, 40, 27912813.

Milfont, T. L., Harré, N., Sibley, C. G., \& Duckitt, J. (2012). The climatechange dilemma: Examining the association between parental status and political party support. Journal of Applied Social Psychology, 42, 2386-2410.

Milfont, T. L., \& Page, E. (2013). A bibliometric review of the first thirty years of the Journal of Environmental Psychology. Psyecology, 4, 195-216.

Milfont, T. L., \& Sibley, C. G. (2011). Exploring the concept of environmental generativity. International Journal of Hispanic Psychology, 4, 21-30.

Milfont, T. L., \& Sibley, C. G. (2012). The big five personality traits and environmental engagement: Associations at the individual and societal level. Journal of Environmental Psychology, 32, 187-195.

Milfont, T. L., Sibley, C. G., \& Duckitt, J. (2010). Testing the moderating role of the components of norm activation on the relationship between values and environmental behavior. Journal of Cross-Cultural Psychology, 41, 124-131.

Milfont, T. L., Wilson, J., \& Diniz, P. (2012). Time perspective and environmental engagement: A meta-analysis. International Journal of Psychology, 47, 325-334. 
Mirosa, M., Lawson, R., \& Gnoth, D. (2013). Linking personal values to energyefficient behaviors in the home. Environment and Behavior, 45, 455-475.

Mostafa, M. M. (2007). Gender differences in Egyptian consumers' green purchase behaviour: the effects of environmental knowledge, concern and attitude. International Journal of Consumer Studies, 31, 220-229.

Musek, J. (1990). Human values in the context of age, gender and personality. Psychologische Beitrage, 32, 36-44.

Nakashima, K. I., Isobe, C., Souma, T., \& Ura, M. (2013). Asymmetric effect of in-group social values on identity with common-identity and common-bond in-groups. Japanese Journal of Psychology, 84, 162-168.

Nemeth, C. J. \& Wachtler, J. (1983). Creative problem solving as a result of majority vs minority influence. European Journal of Social Psychology, 13, 45-55.

Neuman, K. (1986). Personal values and commitment to energy conservation. Environment and Behavior, 18, 53-74.

Nigbur, D., Lyons, E., \& Uzzell, D. (2010). Attitudes, norms, identity and environmental behaviour: Using an expanded theory of planned behaviour to predict participation in a kerbside recycling programme. British Journal of Social Psychology, 49, 259-284.

Nilsson, A., von Borgstede, C., \& Biel, A. (2004). Willingness to accept climate change strategies: The effect of values and norms. Journal of Environmental Psychology, 24, 267-277.

Nordlund, A. M., \& Garvill, J. (2002). Value structures behind proenvironmental behavior. Environment and Behavior, 34, 740-756.

Nordlund, A. \& Westin, K. (2013). Influence of values, beliefs, and age on intention to travel by a new railway line under construction in northern Sweden. Transportation Research Part A, 48, 86-95.

Oreg, S., \& Katz-Gerro, T. (2006). Predicting proenvironmental behavior crossnationally: Values, the Theory of Planned Behavior, and Value-Belief-Norm Theory. Environment and Behavior, 38, 462-483

Organisation for Economic Co-operation and Development (OECD). (2014). OECD Health Statistics. Retrieved from http://www.oecd.org/

Oskamp, S. (2000). Psychological contributions to achieving an ecologically sustainable future for humanity. Journal of Social Issues, 56, 373-390. 
Oskamp, S. (2000). A sustainable future for humanity? How can psychology help? American Psychologist, 55, 496-508.

Ouellette, J. A., Hessling, R., Gibbons, F. X., Reis-Bergan, M., \& Gerrard, M. (2005). Using images to increase exercise behavior: Prototypes versus possible selves. Personality and Social Psychology Bulletin, 31, 610-620.

Ouellette, J. A., \& Wood, W. (1998). Habit and intention in everyday life: The multiple processes by which past behavior predicts future behavior. Psychological Bulletin, 124, 54-74.

Pachauri, R. K. \& Meyer, R. L. (2014). IPCC Climate Change 2014: Synthesis Report. Cambridge, UK: Cambridge University Press.

Pachauri, R. K. \& Reisinger, A. (Eds.) (2007). Contribution of working groups I, II and III to the fourth assessment report of the intergovernmental panel on climate change. Geneva, Switzerland: IPCC. Retrieved from https://www.ipcc.ch/publications_and_data/ar4/syr/en/contents.html

Park, N., \& Yang, A. (2012). Online environmental community members' intention to participate in environmental activities: An application of the theory of planned behavior in the Chinese context. Computers in Human Behavior, 28, 12981306.

Paulhus, D. L. (1998). Interpersonal and intrapsychic adaptiveness of trait selfenhancement: A mixed blessing? Journal of Personality and Social Psychology, 74, 1197-1208.

Peterson, R.A. (2001). On the use of college students in social science research: Insights from a second-order meta-analysis. Journal of Consumer Research, 28, 450461.

Pinto, D. C., Nique, W. M., Añaña, E. d. S., \& Herter, M. M. (2011). Green consumer values: How do personal values influence environmentally responsible water consumption? International Journal of Consumer Studies, 35, 122-131.

Podsiadlowski, A., \& Fox, S. (2011). Collectivist value orientations among four ethnic groups: Collectivism in the New Zealand context. New Zealand Journal of Psychology, 40, 5-18.

Pomery, E. A., Gibbons, F. X., Reis-Bergan, M., \& Gerrard, M. (2009). From willingness to intention: Experience moderates the shift from reactive to reasoned behavior. Personality and Social Psychology Bulletin, 35, 894-908. 
Poortinga, W., Steg, L. \& Vlek, C. (2004). Values, environmental concern, and environmental behavior: A study into household energy use. Environment and Behavior, 36, 70-93.

Rabinovich, A., Morton, T.A., Postmes, T., Verplanken, B. (2012). Collective self and individual choice: the effect of intergroup comparative context on environmental values and behaviour. British Journal of Social Psychology, 51, 551-569.

Reed, A., II, \& Aquino, K. F. (2003). Moral identity and the expanding circle of moral regard toward out-groups. Journal of Personality and Social Psychology, 84, $1270-1286$.

Richard, F. D., Bond Jr., C. F. \& Stokes-Zoota, J. J. (2003). One hundred years of social psychology quantitatively described. Review of General Psychology, 7, 331363.

Ringer, R. (1983). Looking out for \#1. New York: Random House.

Rinne, T., Steel, G. D., \& Fairweather, J. (2012). Hofstede and Shane revisited: The role of power distance and individualism in national-level innovation success. CrossCultural Research: The Journal of Comparative Social Science, 46, 91-108.

Roe, R. A., \& Ester, P. (1999). Values and work: Empirical findings and theoretical perspective. Applied Psychology: An International Review, 48, 1-21.

Rokeach, M. (1973). The nature of human values. New York: Free Press.

Rosenberg, M. (1965). Society and the adolescent self-image. Princeton, NJ: Princeton University Press.

Rosenthal, R., \& DiMatteo, M. R. (2001). Meta analysis: Recent developments in quantitative methods for literature reviews. Annual Review of Psychology, 52, 59-82.

Royne, M. B., Levy, M., \& Martinez, J. (2011). The public health implications of consumers' environmental concern and their willingness to pay for an eco-friendly product. Journal of Consumer Affairs, 45, 329-343.

Sadalla, E. K., \& Krull, J. L. (1995). Self-presentational barriers to resource conservation. Environment and Behavior, 27, 328-353.

Samdahl, D. M., \& Robertson, R. (1989). Social determinants of environmental concern: Specification and test of the model. Environment and Behavior, 21, 57-81.

Saroglou, V., Delpierre, V., \& Dernelle, R. (2004). Values and religiosity: A meta-analysis of studies using Schwartz's model. Personality and Individual Differences, 37, 721-727. 
Sartor, C. E., \& Youniss, J. (2002). The relationship between positive parental involvement and identity achievement during adolescence. Adolescence, 37, 221-234.

Sedikides, C., Gaertner, L., \& Toguchi, Y. (2003). Pancultural selfenhancement, Journal of Personality and Social Psychology, 84, 60-79.

Schultz, P. W. (2001). The structure of environmental concern: Concern for self, other people, and the biosphere. Journal of Environmental Psychology, 21, 327-339.

Schultz, P. W. (2002). Knowledge, education, and household recycling: Examining the knowledge-deficit model of behavior change. In T. Dietz \& P. Stern (Eds.), Education, information, and voluntary measures in environmental protection (pp. 67-82). Washington, DC: National Academy of Sciences.

Schultz, P. W., Gouveia, V. V., Cameron, L. D., Tankha, G., Schmuck, P., \& Franëk, M. (2005). Values and their relationship to environmental concern and conservation behavior. Journal of Cross-Cultural Psychology, 36, 457-475.

Schultz, P. W., \& Kaiser, F. G. (2012). Promoting proenvironmental behavior. In S. Clayton (Ed.) Handbook of environmental and conservation psychology (pp. 556580). Oxford, UK: University Press.

Schultz, P. W., Khazian, A. M., \& Zaleski, A. C. (2008). Using normative social influence to promote conservation among hotel guests. Social Influence, 3, 4-23.

Schultz, P. W., Nolan, J. M., Cialdini, R. B., Goldstein, N. J., \& Griskevicius, V. (2007). The constructive, destructive, and reconstructive power of social norms. Psychological Science, 18, 429-434.

Schultz, P. W., \& Zelezny, L. C. (1998). Values and proenvironmental behavior: A five-country survey. Journal of Cross-Cultural Psychology, 29, 540-558.

Schultz, P. W., \& Zelezny, L. (1999). Values as predictors of environmental attitudes: Evidence for consistency across 14 countries. Journal of Environmental Psychology, 19, 255-265.

Schultz, P. W., \& Zelezny, L. (2003). Reframing environmental messages to be congruent with American values. Research in Human Ecology, 10, 126-136.

Schwartz, S. H. (1968). Awareness of consequences and the influence of moral norms on interpersonal behavior. Sociometry, 31, 355-369.

Schwartz, S. (1992). Universals in the content and structure of values: Theory and empirical tests in 20 countries. In M. Zanna (Ed.), Advances in Experimental Social Psychology (pp. 1-65). New York: Academic Press. 
Schwartz, S. (1994). Are there universal aspects in the content and structure of values? Journal of Social Issues, 50, 19-45.

Schwartz, S. H. (1999). A theory of cultural values and some implications for work. Applied Psychology: An International Review, 48, 23-47.

Schwartz, S. H. (2003). A proposal for measuring value orientations across nations. Questionnaire development report of the European Social Survey. Retrieved from http://naticent02.uuhost.uk.uu.net/questionnaire/chapter_07.doc

Schwartz, S. H. (2004). Mapping and interpreting cultural differences around the world. In V. Vinken, J. Soeters, \& P. Ester (Eds.), Comparing cultures: Dimensions of culture in a comparative perspective (pp. 43-73). Leiden, the Netherlands: Brill.

Schwartz, S. (2006). Basic human values: theory, measurement, and applications. Revue française de sociologie, 47, 929-968.

Schwartz, S. H. (2007). Basic human values: Theory, methods, and application. Risorsa Uomo: Rivista di Psicologia del Lavoro e dell' Organizzazione, 13, 261-283.

Schwartz, S. H. (2010). Basic values: How they motivate and inhibit prosocial behavior. In M. Mikulincer \& P. Shaver (Eds.), Prosocial motives, emotions, and behavior: The better angels of our nature (pp. 221-241). Washington, DC: American Psychological Association Press.

Schwartz, S. H. (2012). An overview of the Schwartz theory of basic values. Online Readings in Psychology and Culture, 2(1). Retrieved from: http://dx.doi.org/10.9707/2307-0919.1116

Schwartz, S. H., \& Bilsky, W. (1990). Toward a psychological structure of human values. Journal of Personality and Social Psychology, 53, 550-562.

Schwartz, S. H., Cieciuch, J., Vecchione, M., Davidov, E., Fischer, R., Beierlein, C., \& Konty, M. (2012). Refining the theory of basic individual values. Journal of Personality and Social Psychology, 103, 663-688.

Schwartz, S. H., \& Howard, J. A. (1980). Explanations of the moderating effect of responsibility denial on the personal norm behavior relationship. Social Psychology Quarterly, 43, 441-446.

Schwartz, S. H., \& Inbar-Saban, N. (1988). Value self-confrontation as a method to aid in weight loss. Journal of Personality and Social Psychology, 54, 396-404.

Schwartz, S. H., Melech, G., Lehmann, A., Burgess, S., Harris, M., \& Owens, V. (2001). Extending the cross-cultural validity of the theory of basic human values with a different method of measurement. Journal of Cross-Cultural Psychology, 32, 519-542. 
Schwartz, S. H., \& Sagiv, L. (1995). Identifying culture-specifics in the content and structure of values. Journal of Cross-Cultural Psychology, 26, 92-116.

Schwarzer, R., \& Jerusalem, M. (1995). Generalized self-efficacy scale. Windsor, UK: NFER-NELSON.

Scott, D., \& Willits, F. K. (1994). Environmental attitudes and behavior: A Pennsylvania survey. Environment and Behavior, 26, 239-260.

Sedikides, C., Gaertner, L., \& Toguchi, Y. (2003). Pancultural selfenhancement. Journal of Personality and Social Psychology, 84, 60-79.

Sibley, C. G. (2014) Sampling procedure and sample details for the New Zealand Attitudes and Values Study. NZAVS Technical Documents, e01.

Smith, P. B., \& Schwartz, S. H. (1997). Values. In J. W. Berry, M. H. Segall, \& C. Kagitçibasi (Eds.), Handbook of cross-cultural psychology (pp. 77-118). Boston, MA: Allyn \& Bacon.

Spence, A., Leygue, C., Bedwell, B., \& O'Malley, C. (2014). Engaging with energy reduction: Does a climate change frame have the potential for achieving broader sustainable behaviour? Journal of Environmental Psychology, 38, 17-28.

Steg, L., Bolderdijk, J. W., Keizer, K., \& Perlaviciute, G. (2014). An integrated framework for encouraging pro-environmental behaviour: The role of values, situational factors and goals. Journal of Environmental Psychology, 38, 104-115.

Steinberg, L., \& Monahan, K. C. (2007). Age differences in resistance to peer influence. Developmental Psychology, 43, 1531-1543.

Stern, P. C. (1992). Psychological dimensions of global environmental change. Annual Review of Psychology, 43, 269-302.

Stern, P. C., \& Dietz, T. (1994). The value basis of environmental concern. Journal of Social Issues, 50, 65-84.

Stern, P., Dietz, T., \& Guagnano, G. A. (1998). A brief inventory of values. Educational and Psychological Measurement, 58, 984-1001.

Stern, P. C., Dietz, T., \& Kalof, L. (1993). Value orientations, gender, and environmental concern. Environment and Behavior, 25, 322-348.

Stern, P. C., Dietz, T., Kalof, L., \& Guagnano, G. A. (1995). Values, beliefs, and proenvironmental Action: Attitude formation toward emergent attitude objects. Journal of Applied Social Psychology, 25, 1611.

Strack, F., \& Deutsch, R. (2004). Reflective and impulsive determinants of social behavior. Personality and Social Psychology Review, 8, 220-247. 
Strathman, A., Gleicher, F., Boninger, D. S., \& Edwards, C. S. (1994). The consideration of future consequences: Weighing immediate and distant outcomes of behavior. Journal of Personality and Social Psychology, 66, 742-752.

Stryker, S., \& Burke, P. J. (2000). The past, present, and future of an Identity Theory. Social Psychology Quarterly Special Issue: The State of Sociological Social Psychology, 63, 284-297.

Tabernero, C., \& Hernández, B. (2011). Self-efficacy and intrinsic motivation guiding environmental behavior. Environment and Behavior, 43, 658-675.

Tangney, J. P., Baumeister, R. F., \& Boone, A. L. (2004). High self-control predicts good adjustment, less pathology, better grades, and interpersonal success. Journal of Personality, 72, 271-322.

Terry, D. J., Hogg, M. A., \& White, K. M. (1999). The theory of planned behaviour: Self-identity, social identity and group norms. British Journal of Social Psychology, 38, 225-244.

Terry, D. J., \& O'Leary, J. E. (1995). The theory of planned behaviour: The effects of perceived behavioural control and self-efficacy. British Journal of Social Psychology, 34, 199-220.

Tuncer, G., Ertepınar, H., Tekkaya, C., \& Sungur, S. (2005). Environmental attitudes of young people in Turkey: Effects of school type and gender. Environmental Education Research, 11, 215-233.

UNFCCC. (2009). Report of the Conference of the Parties on its fifteenth session, held in Copenhagen from 7 to 19 December 2009. Part One: Proceedings. Retrieved from: https://unfccc.int/meetings/copenhagen_dec_2009/meeting/6295/php/view/reports.php UNFCCC. (2010). Report of the Conference of the Parties on its sixteenth session, held in Cancun from 29 November to 10 December 2010. Retrieved from: https://unfccc.int/meetings/cancun_nov_2010/meeting/6266/php/view/reports.php UNFCCC. (2013). Report of the Conference of the Parties on its nineteenth session, held in Warsaw from 11 to 23 November 2013. Retrieved from: http://unfccc.int/documentation/documents/advanced_search/items/6911.php?priref=60 0007786

Urien, B., \& Kilbourne, W. (2011a). Generativity and self-enhancement values in eco-friendly behavioral intentions and environmentally responsible consumption behavior. Psychology \& Marketing, 28, 69-90. 
Vauclair, C. (2010). What is right? What is wrong? And does the answer tell something about culture? An investigation into culture and morality using the values concept. Unpublished doctorate dissertation. Victoria University of Wellington, Wellington, New Zealand.

van de Vijver, F. J. R., \& Poortinga, Y. H. (2002). Structural equivalence in multilevel research. Journal of Cross-Cultural Psychology, 33, 141-156.

Vedder, P., Berry, J., Sabatier, C., \& Sam, D. (2009). The intergenerational transmission of values in national and immigrant families: The role of Zeitgeist. Journal of Youth and Adolescence, 38, 642-653.

Watson, D., Clark, L. A., \& Tellegen, A. (1988). Development and validation of brief measures of positive and negative affect: The PANAS scales. Journal of Personality and Social Psychology, 54, 1063-1070.

Weigel, R. H., \& Weigel, J. (1978). Environmental concern: The development of a measure. Environment and Behavior, 10, 3-15. doi: http://dx.doi.org/10.1177/0013916578101001

Welzel, C., \& Inglehart, R. (2010). Agency, values, and well-being: A human development model. Social Indicators Research, 97, 43-63. doi: http://dx.doi.org/10.1007/s11205-009-9557-z

Welzel, C., Inglehart, R., \& Kligemann, H.-D. (2003). The theory of human development: A cross-cultural analysis. European Journal of Political Research, 42, 341-379.

Whitmarsh, L., \& O'Neill, S. (2010). Green identity, green living? The role of pro-environmental self-identity in determining consistency across diverse proenvironmental behaviours. Journal of Environmental Psychology, 30, 305-314.

Williams, S., \& Schaefer, A. (2013). Small and medium-sized enterprises and sustainability: Managers' values and engagement with environmental and climate change issues. Business Strategy and the Environment, 22, 173-186. doi: http://dx.doi.org/10.1002/bse.1740

Wilson, M. S. (2005). A social-value analysis of postmaterialism. The Journal of Social Psychology, 145, 209-224.

Winerman, L. (2004). Social comparison happens subliminally, and automatically. American Psychological Association, 35, 14.

Zelezny, L. C., \& \& Schultz, P. W. (2000). Promoting environmentalism. Journal of Social Issues, 56, 365-578. 
Yijälä, A., Lönnqvist, J., Jasinskaja-Lahti, I., \& Verkasalo, M. (2012). Values as predictors of anticipated socio-cultural adaptation among potential migrants from Russia to Finland. Journal of Community \& Applied Social Psychology, 22(2), 95-110. doi:http://dx.doi.org/10.1002/casp.1104.

Zimmer, M. R., Stafford, T. F., \& Stafford, M. R. (1994). Green issues:

Dimensions of environmental concern. Journal of Business Research, 30, 63-74. 


\section{APPENDIX A}

\section{Additional Information on Adaptation of GEB scale}

Table A1

Items that Discriminated Participants According to Their Score on GEB Scale

\begin{tabular}{|c|c|c|c|c|c|c|}
\hline \multirow[t]{3}{*}{ Items } & \multicolumn{4}{|c|}{ CRITERIA-GROUPS } & \multicolumn{2}{|c|}{ CONTRASTS } \\
\hline & \multicolumn{2}{|c|}{ Low } & \multicolumn{2}{|c|}{ High } & \multirow[b]{2}{*}{$T$} & \multirow[b]{2}{*}{ d.f. } \\
\hline & $\mathbf{M}$ & SD & $\mathbf{M}$ & SD & & \\
\hline 02. & 3.31 & 0.94 & 3.46 & 0.92 & -1.11 & 203 \\
\hline 03. & 2.49 & 0.85 & 3.49 & 0.69 & $-9.29 *$ & 201 \\
\hline 04. & 1.95 & 0.98 & 3.38 & 0.84 & $-11.20 *$ & 203 \\
\hline 07. & 1.68 & 0.93 & 2.19 & 1.11 & $-3.54 *$ & 191 \\
\hline 08. & 2.59 & 0.92 & 3.37 & 0.82 & $-6.71 *$ & 201 \\
\hline 09. & 2.14 & 0.97 & 2.09 & 1.25 & -0.30 & 180 \\
\hline 10. & 2.53 & 1.01 & 3.01 & 0.99 & $-3.46^{*}$ & 203 \\
\hline 11. & 1.85 & 1.05 & 2.08 & 1.23 & -1.43 & 153 \\
\hline 12. & 1.51 & 0.92 & 1.52 & 1.11 & -0.08 & 189 \\
\hline 17. & 2.23 & 1.04 & 3.09 & 0.99 & $-6.07 *$ & 203 \\
\hline 18. & 2.56 & 0.92 & 3.52 & 0.60 & $-8.93^{*}$ & 185 \\
\hline 19. & 2.60 & 1.05 & 3.45 & 0.80 & $-6.55^{*}$ & 198 \\
\hline 20. & 1.42 & 1.00 & 2.07 & 1.22 & $-4.24 *$ & 188 \\
\hline 21. & 1.18 & 0.93 & 1.62 & 1.15 & -2.98 & 186 \\
\hline 22. & 0.93 & 0.87 & 1.16 & 1.00 & -1.80 & 193 \\
\hline 23. & 1.16 & 0.97 & 1.59 & 1.10 & $-2.99 *$ & 194 \\
\hline 24. & 1.15 & 0.98 & 2.03 & 1.45 & $-5.07 *$ & 169 \\
\hline 25. & 2.16 & 0.96 & 2.98 & 1.00 & $-5.99 *$ & 200 \\
\hline 26. & 1.54 & 0.88 & 1.34 & 1.00 & 1.52 & 193 \\
\hline 27. & 2.29 & 0.96 & 3.23 & 0.88 & $-7.39 *$ & 204 \\
\hline 36. & 2.29 & 1.02 & 3.27 & 0.97 & $-7.04^{*}$ & 204 \\
\hline 37. & 2.54 & 0.91 & 3.39 & 0.74 & $-7.38^{*}$ & 202 \\
\hline 38. & 1.62 & 1.01 & 2.36 & 1.26 & $-4.59 *$ & 186 \\
\hline 39. & 2.06 & 0.95 & 3.21 & 0.98 & $-8.63^{*}$ & 201 \\
\hline 40. & 1.51 & 0.84 & 1.46 & 1.09 & 0.37 & 204 \\
\hline 41. & 2.46 & 0.95 & 3.21 & 0.85 & $-5.98^{*}$ & 204 \\
\hline 42. & 1.40 & 0.76 & 1.29 & 0.98 & 0.68 & 202 \\
\hline 43. & 2.44 & 0.92 & 3.09 & 0.94 & $-5.05^{*}$ & 201 \\
\hline 44. & 2.20 & 0.85 & 2.92 & 0.94 & $-5.71 *$ & 196 \\
\hline 45. & 3.05 & 0.86 & 3.62 & 0.67 & $-5.41^{*}$ & 199 \\
\hline 46. & 1.56 & 0.82 & 2.62 & 1.05 & $-7.99 *$ & 183 \\
\hline 47. & 1.56 & 0.92 & 2.30 & 1.15 & $-5.00 *$ & 185 \\
\hline 48. & 1.88 & 0.86 & 3.08 & 0.85 & $-10.10 *$ & 203 \\
\hline 49. & 1.76 & 0.96 & 2.99 & 0.88 & $-9.57^{*}$ & 204 \\
\hline 50. & 2.36 & 0.79 & 3.36 & 0.68 & $-9.73^{*}$ & 203 \\
\hline
\end{tabular}

* Discriminant Item, $p<.001$ 


\section{Table A2}

Exploratory Factorial Analysis for GEB Scale

\begin{tabular}{|c|c|c|}
\hline Items* & $\begin{array}{l}\text { Component } \\
\text { loading }\end{array}$ & $\begin{array}{c}\text { Corrected } \\
\text { Item-Total } \\
\text { Correlation }\end{array}$ \\
\hline 50. Buy products in refillable packages & .76 & .72 \\
\hline 48. Look into the pros and cons of having a private source of solar power & .75 & .73 \\
\hline 49. Request an estimate on having solar power installed & .75 & .72 \\
\hline 18. Use renewable energy sources & .72 & .70 \\
\hline 32. Talk with friends about problems related to the environment & .71 & .93 \\
\hline 37. Reuse your shopping bags & .70 & .93 \\
\hline 49. Read about environmental issues & 69 & .93 \\
\hline 3. Own energy efficient household devices & .68 & 63 \\
\hline 5. Bring empty bottles to a recycling bin & .68 & .93 \\
\hline 4. Collect and recycle used paper & 67 & .93 \\
\hline 30. Buy milk in returnable bottles & 67 & .64 \\
\hline 29. Own a fuel-efficient automobile (less than 71 per $100 \mathrm{~km}$; i.e., less than 3 gallons per 100 miles) & .64 & .93 \\
\hline 33. Be a member of an environmental organization & .64 & 62 \\
\hline 44. Buy domestically grown wooden furniture & .63 & .59 \\
\hline 41. Buy seasonal produce & .62 & .57 \\
\hline $\begin{array}{l}\text { 39. Use public transportation or ride a bike, when possible in nearby areas (around } 30 \mathrm{~km} \text {; around } 20 \\
\text { miles) }\end{array}$ & 61 & .57 \\
\hline 36. Ride a bicycle or take public transportation to work or school & .60 & .54 \\
\hline 17. Buy solar panels to produce energy & .59 & .55 \\
\hline 19. Turn down the heat when I leave my apartment for more than $4 \mathrm{~h}$ during winter & .57 & .50 \\
\hline 43. Buy meat and produce with eco-labels & .56 & .52 \\
\hline 27. Drive in such a way as to keep my fuel consumption as low as possible & .56 & .52 \\
\hline 45. Leave the place as clean as it was originally after a picnic & .55 & .47 \\
\hline 47. Boycott companies with an unecological background & .52 & .52 \\
\hline 35. Contribute financially to environmental organizations & .56 & .50 \\
\hline 25. Be a member of a carpool & .50 & .45 \\
\hline 34. Point out to someone his or her unecological behaviour & .49 & .47 \\
\hline 8. Wait until you have a full load before doing your laundry & .44 & .38 \\
\hline 38. Drive on freeways at speeds under 100 k.p.h. (1/462.5 m.p.h.) & .40 & .38 \\
\hline Number of items & \multicolumn{2}{|c|}{28} \\
\hline Eigenvalue & \multicolumn{2}{|c|}{11.27} \\
\hline$\%$ variance explained & \multicolumn{2}{|c|}{27.49} \\
\hline Cronbach's Alpha & \multicolumn{2}{|c|}{.94} \\
\hline
\end{tabular}

* Item displayed according to component loading. Items in grated grey were part of the second

half of the scale (GEB-2) 


\section{APPENDIX B}

Questionnaire for Pilot Study - Adaptation of General Environmental Behaviour (GEB)

Scale

TE WHARE WĀNANGA O TE ŪPOKO O TE IKA A MĀUI

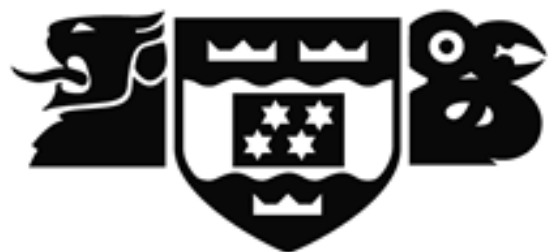

\section{PART 1. General questions about the environment}

Please indicate the extent to which you would be willing to engage in the following behaviours:

\begin{tabular}{|c|c|c|c|c|c|c|c|}
\hline $\begin{array}{c}0 \\
\text { Not at all } \\
\text { willing }\end{array}$ & $\begin{array}{c}1 \\
\text { A little } \\
\text { willing }\end{array}$ & $\begin{array}{c}2 \\
\begin{array}{c}\text { Moderately } \\
\text { willing }\end{array} \\
\end{array}$ & $\begin{array}{c}3 \\
\text { Very willing }\end{array}$ & \multicolumn{4}{|c|}{$\begin{array}{c}4 \\
\text { Extremely } \\
\text { willing } \\
\end{array}$} \\
\hline \multicolumn{4}{|c|}{ Put dead batteries in the garbage. } & & 123 & & \\
\hline \multicolumn{4}{|c|}{ Dispose of leftovers in the toilet after meals. } & 0 & 123 & & \\
\hline \multicolumn{4}{|c|}{ Own energy efficient household devices. } & 0 & 123 & & \\
\hline \multicolumn{4}{|c|}{ Collect and recycle used paper. } & 0 & 123 & & \\
\hline \multicolumn{4}{|c|}{ Bring empty bottles to a recycling bin. } & 0 & $12:$ & & \\
\hline \multicolumn{4}{|c|}{ Prefer to shower rather than to take a bath. } & 0 & $12:$ & & \\
\hline \multicolumn{4}{|c|}{$\begin{array}{l}\text { Keep the heat on in the winter, so that you do not have to } \\
\text { wear a sweater. }\end{array}$} & 0 & 12 & & 4 \\
\hline \multicolumn{4}{|c|}{ Wait until you have a full load before doing your laundry. } & 0 & $12:$ & & \\
\hline \multicolumn{4}{|c|}{$\begin{array}{l}\text { Leave the windows open, in the winter, for long periods of } \\
\text { time to let fresh air. }\end{array}$} & 0 & 12 & & 4 \\
\hline \multicolumn{4}{|c|}{ Wash dirty clothes without prewashing. } & 0 & 123 & & 4 \\
\hline \multicolumn{4}{|c|}{ Use fabric softener with your laundry. } & 0 & $12:$ & & 4 \\
\hline \multicolumn{4}{|c|}{ Use an oven-cleaning spray to clean your oven. } & 0 & $12:$ & & 4 \\
\hline \multicolumn{4}{|c|}{ Kill insects with a chemical insecticide. } & 0 & 12 & & 4 \\
\hline \multicolumn{4}{|c|}{ Use a chemical air freshener in the bathroom. } & 0 & 12 & & 4 \\
\hline \multicolumn{4}{|c|}{ Have the towels changed daily in hotels. } & 0 & 12 & & 4 \\
\hline \multicolumn{4}{|c|}{ Use a clothes dryer. } & 0 & 12 & & 4 \\
\hline \multicolumn{4}{|c|}{ Buy solar panels to produce energy. } & 0 & 12 & & 4 \\
\hline \multicolumn{4}{|c|}{ Use renewable energy sources. } & 0 & 12 & & 4 \\
\hline \multicolumn{4}{|c|}{$\begin{array}{l}\text { Turn down the heat when I leave my apartment for more than } \\
4 \mathrm{~h} \text { during winter. }\end{array}$} & 0 & 12 & & 4 \\
\hline
\end{tabular}


Drive my car in or into the city.

$\begin{array}{lllll}0 & 1 & 2 & 3 & 4\end{array}$

Keep the engine running while waiting in front of a railroad crossing or in a traffic jam.

$\begin{array}{lllll}0 & 1 & 2 & 3 & 4\end{array}$

Keep the engine running at red traffic lights.

$\begin{array}{lllll}0 & 1 & 2 & 3 & 4\end{array}$

Drive to where you want to start your hikes.

$\begin{array}{lllll}0 & 1 & 2 & 3 & 4\end{array}$

Refrain from owning a car.

$\begin{array}{lllll}0 & 1 & 2 & 3 & 4\end{array}$

Be a member of a carpool.

$\begin{array}{lllll}0 & 1 & 2 & 3 & 4\end{array}$

Buy beverages in cans.

$\begin{array}{lllll}0 & 1 & 2 & 3 & 4\end{array}$

Drive in such a way as to keep my fuel consumption as low as possible.

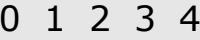

Take a plastic bag if it is offered in a store.

$\begin{array}{lllll}0 & 1 & 2 & 3 & 4\end{array}$

Own a fuel-efficient automobile (less than 7 I per 100 km;

i.e., less than 3 gallons per 100 miles).

$\begin{array}{lllll}0 & 1 & 2 & 3 & 4\end{array}$

Buy milk in returnable bottles.

$\begin{array}{lllll}0 & 1 & 2 & 3 & 4\end{array}$

Take an airplane for longer journeys (more than $6 \mathrm{~h}$ ).

$\begin{array}{lllll}0 & 1 & 2 & 3 & 4\end{array}$

Talk with friends about problems related to the environment.

$\begin{array}{lllll}0 & 1 & 2 & 3 & 4\end{array}$

Be a member of an environmental organization.

$\begin{array}{lllll}0 & 1 & 2 & 3 & 4\end{array}$

Point out to someone his or her unecological behaviour.

$\begin{array}{lllll}0 & 1 & 2 & 3 & 4\end{array}$

Contribute financially to environmental organizations.

$\begin{array}{lllll}0 & 1 & 2 & 3 & 4\end{array}$

Ride a bicycle or take public transportation to work or school.

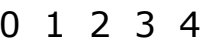

Reuse your shopping bags.

$\begin{array}{lllll}0 & 1 & 2 & 3 & 4\end{array}$

Drive on freeways at speeds under 100 k.p.h. (1/462.5 m.p.h.).

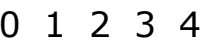

Use public transportation or ride a bike, when possible in nearby areas (around $30 \mathrm{~km}$; around 20 miles).

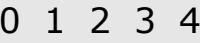

Buy convenience foods.

$\begin{array}{lllll}0 & 1 & 2 & 3 & 4\end{array}$

Buy seasonal produce.

$\begin{array}{lllll}0 & 1 & 2 & 3 & 4\end{array}$

Buy bleached and colored toilet paper.

$\begin{array}{lllll}0 & 1 & 2 & 3 & 4\end{array}$

Buy meat and produce with eco-labels.

$\begin{array}{lllll}0 & 1 & 2 & 3 & 4\end{array}$

Buy domestically grown wooden furniture.

$\begin{array}{lllll}0 & 1 & 2 & 3 & 4\end{array}$

Leave the place as clean as it was originally after a picnic.

$\begin{array}{lllll}0 & 1 & 2 & 3 & 4\end{array}$

Read about environmental issues.

$\begin{array}{lllll}0 & 1 & 2 & 3 & 4\end{array}$

Boycott companies with an unecological background.

$\begin{array}{lllll}0 & 1 & 2 & 3 & 4\end{array}$

Look into the pros and cons of having a private source of solar power.

$\begin{array}{lllll}0 & 1 & 2 & 3 & 4\end{array}$

Request an estimate on having solar power installed.

$\begin{array}{lllll}0 & 1 & 2 & 3 & 4\end{array}$

Buy products in refillable packages.

$\begin{array}{lllll}0 & 1 & 2 & 3 & 4\end{array}$ 


\section{PART 2. Personal values}

We would like to know how important each value is as a guiding principle in your life. Please rate each value below using the following nine-point scale.

\begin{tabular}{|c|c|c|c|c|c|c|c|}
\hline $\begin{array}{c}-1 \\
\text { Opposed to } \\
\text { my values }\end{array}$ & $\begin{array}{c}0 \\
\text { Not } \\
\text { important }\end{array}$ & $\mathbf{1}$ & \begin{tabular}{|c|c|}
2 & 3 \\
Importa \\
nt
\end{tabular} & \begin{tabular}{l|l}
4 & 5
\end{tabular} & $\begin{array}{c}6 \\
\text { Very } \\
\text { important }\end{array}$ & $\begin{array}{c}7 \\
\text { Of supreme } \\
\text { importance }\end{array}$ & \\
\hline \multicolumn{6}{|c|}{ EQUALITY (equal opportunity for all) } & -10123 & 4567 \\
\hline \multicolumn{6}{|c|}{ SOCIAL POWER (control over others) } & $\begin{array}{lllll}-1 & 0 & 1 & 2 & 3\end{array}$ & 4567 \\
\hline \multicolumn{6}{|c|}{ WEALTH (material possessions, money) } & $\begin{array}{lllll}-1 & 0 & 1 & 2 & 3\end{array}$ & 4567 \\
\hline \multicolumn{6}{|c|}{ A WORLD OF PEACE (free of war and conflict) } & $\begin{array}{lllll}-1 & 0 & 1 & 2 & 3\end{array}$ & 4567 \\
\hline \multicolumn{6}{|c|}{ SOCIAL RECOGNITION (respect, approval by others) } & $\begin{array}{lllll}-1 & 0 & 1 & 2 & 3\end{array}$ & 4567 \\
\hline \multicolumn{6}{|c|}{ AUTHORITY (the right to lead or command) } & $\begin{array}{lllll}-10 & 1 & 2 & 3\end{array}$ & 4567 \\
\hline \multicolumn{6}{|c|}{ UNITY WITH NATURE (fitting into nature) } & $\begin{array}{lllll}-1 & 0 & 1 & 2 & 3\end{array}$ & 4567 \\
\hline \multicolumn{6}{|c|}{ SOCIAL JUSTICE (correcting injustice, care for the weak) } & $\begin{array}{lllll}-10 & 1 & 2 & 3\end{array}$ & 4567 \\
\hline \multicolumn{6}{|c|}{ LOYAL (faithful to my friends, group) } & $\begin{array}{lllll}-1 & 0 & 1 & 2 & 3\end{array}$ & 4567 \\
\hline \multicolumn{6}{|c|}{ AMBITIOUS (hardworking, aspiring) } & $\begin{array}{lllll}-1 & 0 & 1 & 2 & 3\end{array}$ & 4567 \\
\hline \multicolumn{6}{|c|}{ BROAD-MINDED (tolerant of different ideas and beliefs) } & $\begin{array}{lllll}-1 & 0 & 1 & 2 & 3\end{array}$ & 4567 \\
\hline \multicolumn{6}{|c|}{ INFLUENTIAL (having an impact on people and events) } & $\begin{array}{lllll}-10 & 1 & 2 & 3\end{array}$ & 4567 \\
\hline \multicolumn{6}{|c|}{ PROTECTING THE ENVIRONMENT (preserving nature) } & $\begin{array}{lllll}-1 & 0 & 1 & 2 & 3\end{array}$ & 4567 \\
\hline \multicolumn{6}{|c|}{ CAPABLE (competent, effective, efficient) } & $\begin{array}{lllll}-10 & 1 & 2 & 3\end{array}$ & 4567 \\
\hline \multicolumn{6}{|c|}{ HONEST (genuine, sincere) } & $\begin{array}{lllll}-1 & 0 & 1 & 2 & 3\end{array}$ & 4567 \\
\hline \multicolumn{6}{|c|}{ HELPFUL (working for the welfare of others) } & $\begin{array}{lllll}-1 & 0 & 1 & 2 & 3\end{array}$ & 4567 \\
\hline \multicolumn{6}{|c|}{ FORGIVING (willing to pardon others) } & $\begin{array}{lllll}-1 & 0 & 1 & 2 & 3\end{array}$ & 4567 \\
\hline \multicolumn{6}{|c|}{ RESPECTING THE EARTH (harmony with other species) } & $\begin{array}{lllll}-1 & 0 & 1 & 2 & 3\end{array}$ & 4567 \\
\hline \multicolumn{6}{|c|}{ UCESSFUL (achieving goals) } & $\begin{array}{lllll}-1 & 0 & 1 & 2 & 3\end{array}$ & 4567 \\
\hline
\end{tabular}




\section{PART 3. Background questions}

(Please remember that your responses are confidential)

1. How old are you? years.
2. What is your gender?

1. Female

2. Male

3. Are you a member of any environmental organisation (e.g., Greenpeace)?

4. Were you born in New Zealand?
1. Yes
2. No

2. No

5. Which ethnic group do you belong to? Please indicate the group you most strongly identify with.
1. New Zealand European (Pākehā)
4. Māori
2. Pacific Nations
5. Asian
3. Indian
6. Other (please specify):

\section{Thank you for your time! :}




\section{Information Sheet for Pre-test (mass testing) - Experiment 1}

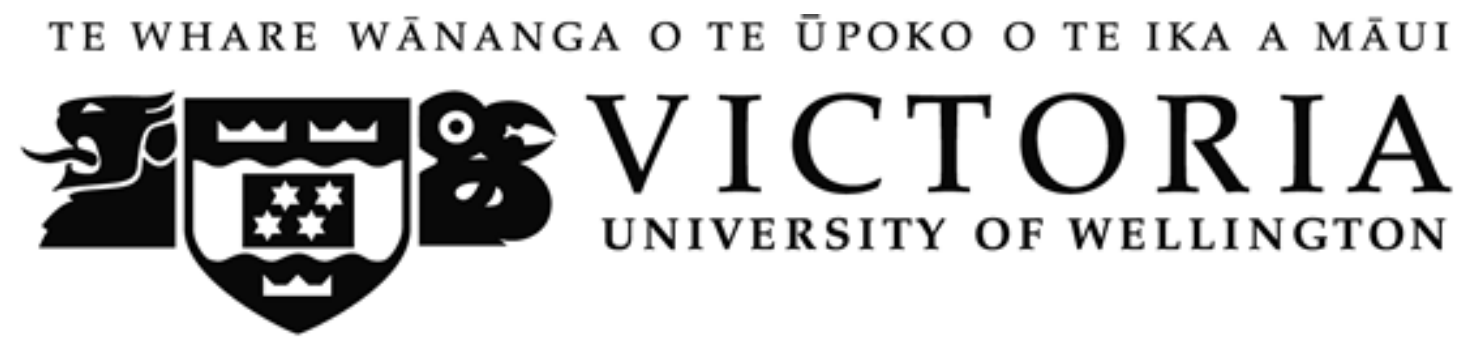

Pollyane Diniz

PhD Student

School of Psychology

Victoria University of Wellington

Pollyane.Diniz@vuw.ac.nz
Dr. Taciano L. Milfont

Senior Lecturer

School of Psychology

Victoria University of Wellington

Taciano.Milfont@vuw.ac.nz

Phone: 463-6398

\author{
Dr. John McClure \\ Professor \\ School of Psychology \\ Victoria University of Wellington \\ john.mcclure@vuw.ac.nz \\ Phone: 4635402
}

\section{What is the purpose of this research?}

This research consists of a questionnaire that asks about opinions, attitudes, and behaviours on a number of social issues that are relevant to our future. The goal is to understand the opinions towards these issues.

\section{Who is conducting the research?}

Pollyane Diniz is a PhD student. Dr. Taciano Milfont and Dr. John McClure are supervising this project. This research has been approved by the School of Psychology Human Ethics Committee under delegated authority by Victoria University of Wellington Human Ethics Committee

\section{What is involved if you agree to participate?}

- If you agree to participate in this study you will be asked to complete a short survey that will ask you about your identity, specific behaviours, and some demographic questions.

- We anticipate that the survey will take you no more than 5 minutes to complete.

- There are no risks in this study that arise from your participation. During the research you are free to withdraw at any point before your survey has been completed.

- Because we are interested in the responses each person makes without the influence of others, we ask that you not discuss this study with your classmates until sometime towards the end of the semester, when we will send an email to you at your VUW email address, telling you about the results. A debriefing sheet will also be posted on blackboard.

\section{Privacy and Confidentiality}

- This survey is completely anonymous. Please do not put your name anywhere on the survey.

- You will never be identified in my research project or in any other presentation or publication. The information you provide will be coded by number only.

- We will keep your data for at least five years after publication (first publication is expected at the end of 2013), and then it will be destroyed.

- In accordance with the requirements of some scientific journals and organisations, your coded survey may be shared with other competent researchers.

- Your coded data may be used in other, related studies.

- A copy of the coded data will remain in the custody of Pollyane Diniz. 


\section{What happens to the information that you provide?}

- The data you provide may be used for one or more of the following purposes:

The overall findings may be submitted for publication in a scientific journal, or presented at scientific conferences.

The overall findings may form part of a $\mathrm{PhD}$ thesis that will be submitted for assessment.

\section{Statement of consent}

I have read the information about this research and any questions I wanted to ask have been answered to my satisfaction.

I agree to participate in this research. I understand that I can withdraw my consent at any time, without penalty, prior to the end of my participation.

Name:

Signature:

Date:

Student ID:

Please write down your email address if you want to be notified about the results of the study. 


\section{APPENDIX D}

Questionnaire for Pre-test (mass testing) - Experiment 1

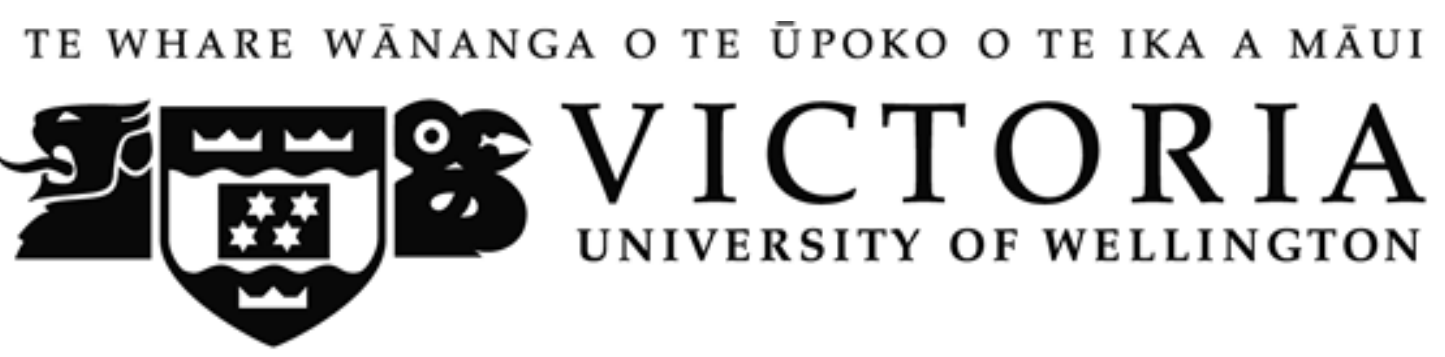

\section{PART 1. General questions about the environment (GEB 1)}

Please indicate the extent to which you would be willing to engage in the following behaviours:

\begin{tabular}{|c|c|c|c|c|}
\hline $\begin{array}{c}0 \\
\text { Not at all } \\
\text { willing }\end{array}$ & $\begin{array}{c}1 \\
\text { A little } \\
\text { willing }\end{array}$ & $\begin{array}{c}2 \\
\text { Moderately } \\
\text { willing }\end{array}$ & $\begin{array}{c}3 \\
\text { Very } \\
\text { willing }\end{array}$ & $\begin{array}{c}4 \\
\text { Extremely } \\
\text { willing }\end{array}$ \\
\hline \multicolumn{4}{|c|}{ Reuse your shopping bags. } & 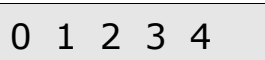 \\
\hline \multicolumn{4}{|c|}{ Bring empty bottles to a recycling bin. } & 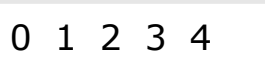 \\
\hline \multicolumn{4}{|c|}{ Buy seasonal produce. } & 012234 \\
\hline \multicolumn{4}{|c|}{$\begin{array}{l}\text { Drive in such a way as to keep my fuel consumption as low as } \\
\text { possible. }\end{array}$} & 01234 \\
\hline \multicolumn{4}{|c|}{ Own energy efficient household devices. } & 01234 \\
\hline \multicolumn{4}{|c|}{ Buy domestically grown wooden furniture. } & 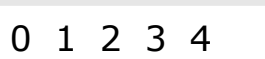 \\
\hline \multicolumn{4}{|c|}{ Buy meat and produce with eco-labels. } & $0 \begin{array}{llll}0 & 1 & 2 & 3\end{array}$ \\
\hline \multicolumn{4}{|c|}{$\begin{array}{l}\text { Look into the pros and cons of having a private source of solar } \\
\text { power. }\end{array}$} & 01234 \\
\hline \multicolumn{4}{|c|}{ Talk with friends about problems related to the environment. } & 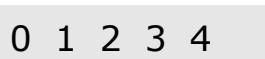 \\
\hline \multicolumn{4}{|c|}{$\begin{array}{l}\text { Own a fuel-efficient automobile (less than } 7 \text { I per } 100 \mathrm{~km} \text {; i.e., } \\
\text { less than } 3 \text { gallons per } 100 \text { miles). }\end{array}$} & 01234 \\
\hline \multicolumn{4}{|c|}{ Buy milk in returnable bottles. } & $0 \begin{array}{llll}0 & 1 & 2 & 3\end{array}$ \\
\hline \multicolumn{4}{|c|}{ Point out to someone his or her unecological behaviour. } & 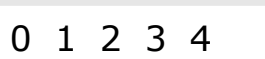 \\
\hline \multicolumn{4}{|c|}{ Use renewable energy sources. } & 012234 \\
\hline \multicolumn{4}{|c|}{ Be a member of a carpool. } & 012234 \\
\hline
\end{tabular}




\section{PART 2. Personal values (V1)}

We would like to know how important each value is as a guiding principle in your life. Below is a list of 16 values in alphabetical order. We are interested in finding out the relative importance of theses values to you.

Study the list carefully. Then place a 1 next to the value which is most important to you, place a 2 next to the second values which is second most important, etc. The values which is least important should be ranked 16 .

When you completed ranking all the values, go back and check over your list. Feel free to make changes. Please take all the time you need to think about this, so that the end result truly represents your values.

A VARIED LIFE (filled with challenge, novelty, and change)

A WORLD OF PEACE (free of war and conflict)

AMBITIOUS (hardworking, aspiring)

AN EXCITING LIFE (stimulating experiences)

CURIOUS (interested in everything, exploring)

DETACHMENT (from worldly concerns)

EQUALITY (equal opportunity for all)

HELPFUL (working for the welfare of others)

INDEPENDENT (self-reliant, self-sufficient)

LOYAL (faithful to my friends, group)

MODERATE (avoiding extremes of feeling and action)

POLITENES (courtesy, good manners)

RESPECT FOR TRADITION (preservation of time-honoured customs)

SOCIAL POWER (control over others, dominance)

SOCIAL RECOGNITION (respect, approval by others)

SUCCESSFUL (achieving goals) 
PART 3. Group Identification (note that this measure will also be included in the questionnaire for Experiment 2 with 'VUW students' being replaced by 'New Zealanders)

Please select the figure below that best describes your relationship with VUW students.

\section{How interconnected are you with Victoria University Students?}

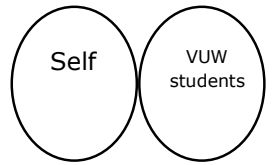

1

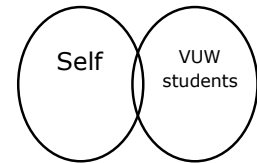

2

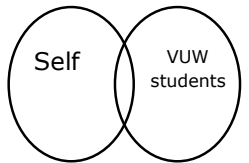

3

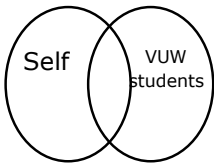

4

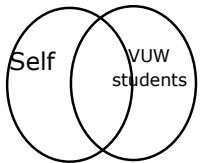

5

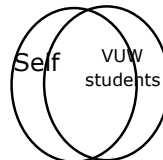

6

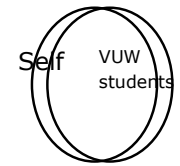

7

Now, please think about you as a student (e.g. VUW student) and respond to the following statements on the basis of how you feel about being a VUW students. Please read each statement carefully, and respond by using the following scale from 1 to 7 :

\begin{tabular}{|c|c|c|c|c|c|c|c|}
\hline $\begin{array}{c}1 \\
\text { Strongly } \\
\text { Disagree }\end{array}$ & $\begin{array}{c}2 \\
\text { Disagree }\end{array}$ & $\begin{array}{c}3 \\
\text { Disagree } \\
\text { Somewhat }\end{array}$ & $\begin{array}{c}4 \\
\text { Neutral }\end{array}$ & $\begin{array}{r}\text { Ag } \\
\text { Some }\end{array}$ & $\begin{array}{l}\text { ee } \\
\text { what }\end{array}$ & $\begin{array}{c}6 \\
\text { Agree }\end{array}$ & $\begin{array}{c}7 \\
\text { Strongly } \\
\text { Agree }\end{array}$ \\
\hline \multicolumn{8}{|c|}{ I often regret being VUW student. } \\
\hline \multicolumn{4}{|c|}{ In general, I'm glad to be part of VUW students. } & & 123 & 456 & \\
\hline \multicolumn{5}{|c|}{ Overall, I often feel that being VUW student is not worthwhile } & 123 & 456 & \\
\hline \multicolumn{5}{|c|}{ I feel good about belonging to VUW students. } & 123 & 456 & \\
\hline \multicolumn{5}{|c|}{ Overall, VUW students are considered good by others. } & 123 & 456 & \\
\hline \multicolumn{5}{|c|}{$\begin{array}{l}\text { Most people consider VUW students, on the average, to be } \\
\text { more ineffective than other ethnic groups. }\end{array}$} & 123 & 456 & \\
\hline \multicolumn{5}{|c|}{ In general, others respect VUW students. } & 123 & 456 & \\
\hline \multicolumn{5}{|c|}{ In general, others think that VUW students are unworthy. } & 123 & 456 & \\
\hline \multicolumn{5}{|c|}{$\begin{array}{l}\text { Overall, being VUW students has very little to do with how I } \\
\text { feel about myself. }\end{array}$} & 123 & 456 & 7 \\
\hline \multicolumn{5}{|c|}{$\begin{array}{l}\text { Belonging to VUW students is an important reflection of who I } \\
\text { am. }\end{array}$} & 123 & 456 & 7 \\
\hline \multicolumn{5}{|c|}{$\begin{array}{l}\text { Being VUW student is unimportant to my sense of what kind of } \\
\text { a person I am. }\end{array}$} & 123 & 456 & 7 \\
\hline \multicolumn{5}{|c|}{$\begin{array}{l}\text { In general, being part of VUW students is an important part of } \\
\text { my self image. }\end{array}$} & 123 & 456 & 7 \\
\hline
\end{tabular}




\section{PART 4. Background questions}

\section{(Please remember that your responses are confidential)}

1. How old are you? years.
2. What is your gender?

1. Female

2. Male
3. Are you a member of any environmental organisation
1. Yes
2. No

4. Were you born in New Zealand?

1. Yes

2. No

5. Which ethnic group do you belong to? Please indicate the group you most strongly identify with.

1. New Zealand European (Pākehā) 4. Māori

2. Pacific Nations

5. Asian

3. Indian

6. Other (please specify):

Thank you for your time! :) 


\section{Information Sheet for Post-test - Experiment 1}

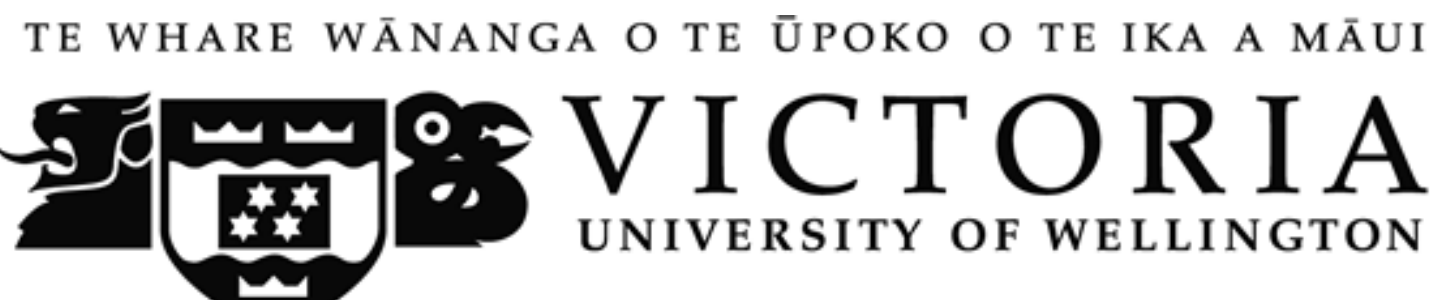

Pollyane Diniz

PhD Candidate

School of Psychology

Victoria University of Wellington

Pollyane.Diniz@vuw.ac.nz

\section{Dr. Taciano L. Milfont}

Senior Lecturer

School of Psychology

Victoria University of Wellington

Taciano.Milfont@vuw.ac.nz

Phone: 463-6398
Dr. John McClure

Professor

School of Psychology

Victoria University of Wellington john.mcclure@vuw.ac.nz

Phone: 4635402

\section{What is the purpose of this research?}

This research consists of a questionnaire that asks about opinions, attitudes, and behaviours on a number of social issues that are relevant to our future. The goal is to understand the opinions towards these issues.

\section{Who is conducting the research?}

Pollyane Diniz is a PhD student. Dr. Taciano Milfont and Dr. John McClure are supervising this project. This research has been approved by the School of Psychology Human Ethics Committee under delegated authority by Victoria University of Wellington Human Ethics Committee.

\section{What is involved if you agree to participate?}

- You will participate in one session today. If you agree to participate in this study you will be asked to complete a short survey. It will involve answering a second part of a questionnaire that you answered in the mass testing and also it will ask you about your mood, well-being, identity and specific behaviours. The questionnaire will take around 30 minutes to complete. You will be rewarded with .5 IPRP credit for taking part in this study.

- There are no risks in this study that arise from your participation. During the research you are free to withdraw at any point before your survey has been completed.

- Because we are interested in the responses each person makes without the influence of others, we ask that you not discuss this study with your classmates until sometime towards the end of the semester, when all data has been collected and we will send you an email with a debriefing sheet. If you would like to know the results of this study, they will be available at the end of the semester via email upon request.

\section{Privacy and Confidentiality}

- This survey is completely confidential. The information sheet will be separate from your individualized questionnaire. 
- You will never be identified in my research project or in any other presentation or publication. The information you provide will be coded by number only.

- We will keep your coded data for at least five years after publication (first publication is expected at the end of 2013), and then it will be destroyed.

- In accordance with the requirements of some scientific journals and organizations, your coded survey may be shared with other competent researchers.

- Your coded data may be used in other, related studies.

- A copy of the coded data will remain in the custody of Pollyane Diniz.

\section{What happens to the information that you provide?}

- The data you provide may be used for one or more of the following purposes:

The overall findings may be submitted for publication in a scientific journal, or presented at scientific conferences. The overall findings may form part of a $\mathrm{PhD}$ thesis that will be submitted for assessment.

\section{Statement of consent}

I have read the information about this research and any questions I wanted to ask have been answered to my satisfaction. I agree to participate in this research. I understand that I can withdraw my consent at any time, without penalty, prior to the end of my participation.

Name:

Email address:

Signature:

Date: 


\section{APPENDIX F}

\section{Self-transcendence Values Condition - Experiment 1}

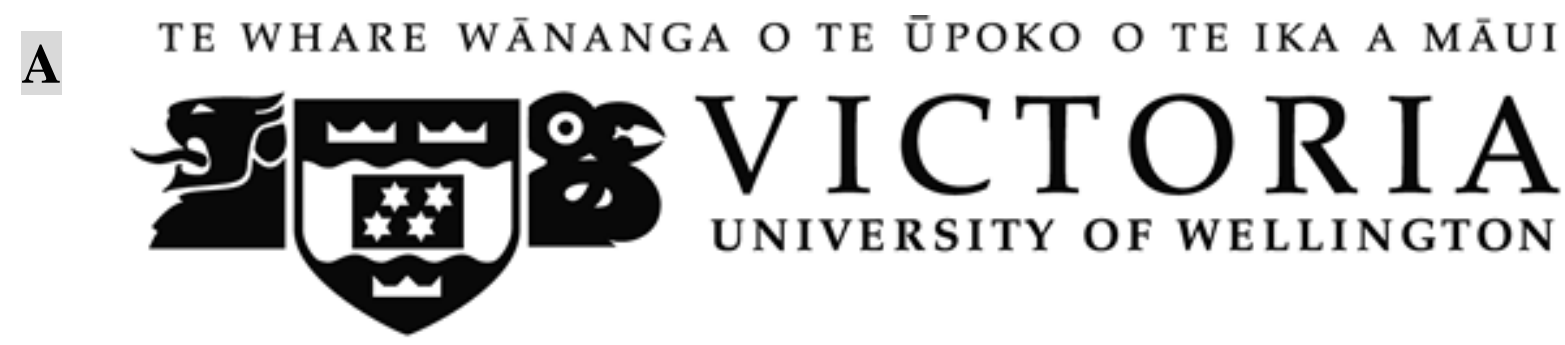

Part 1. Personal values

Now, I would like to tell you some things we have already found about the value systems of Victoria University students. I am sure that many of you would like to know what they are.

The same value system scale that you answered previously in the mass testing was filled out by 298 students in Psychology 122 . The responses of these students were obtained and averaged together. The table below shows the results.

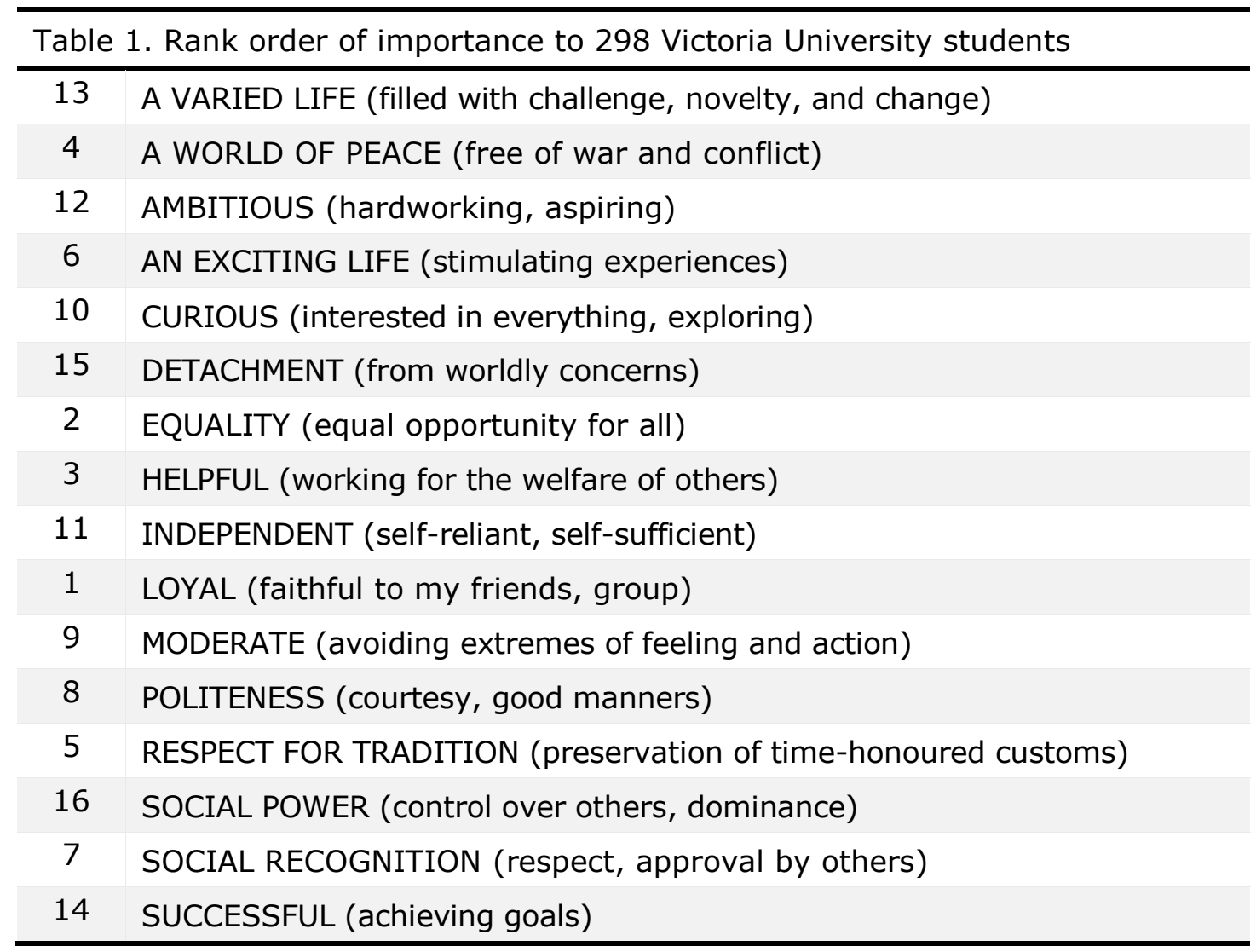


You have your own rankings (from the mass testing) at the end of this questionnaire. Feel free to spend a few minutes comparing your own rankings with those of the 298 students, shown in Table 1.

Please identify the four most highly ranked values in the Victoria University students ranking and write the names of the values in the predetermined column below.

The four most highly ranked values for Victoria University students were:

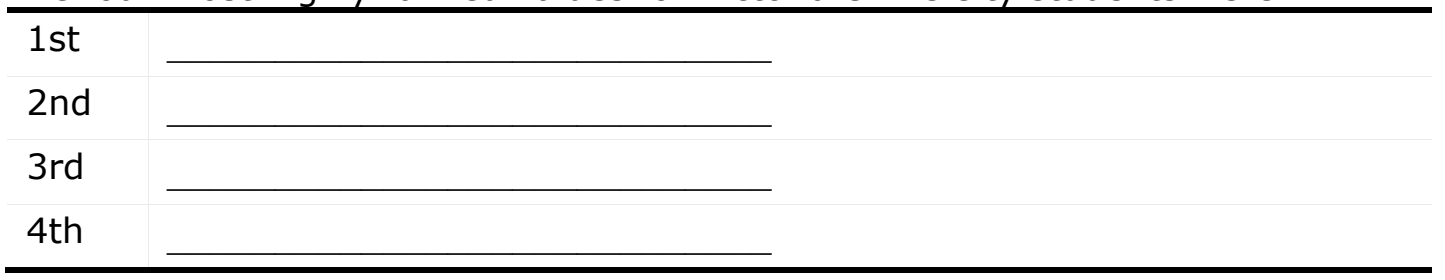

Now, do the same task for your own value rankings. Please identify the four most highly ranked values in your own ranking and write the names of the values in the predetermined column below.

The four most highly ranked values for you were:

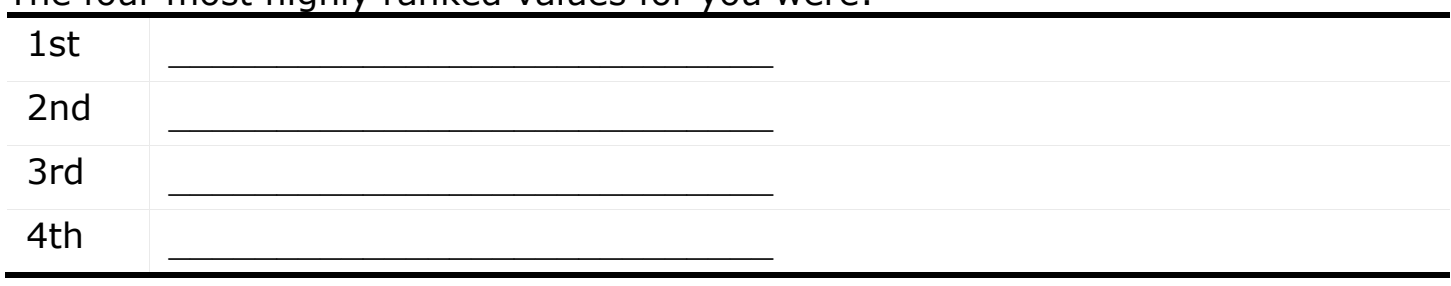

Can you recognize any similarities and differences between your value rankings and the student's value rankings? Please write down your comments.

Below we have an explanation about the first four preferred values of Victoria University students and about the characteristics of people who rank those values as their most preferred values. Please spend some time reading through the following explanation.

The average of the students' value ranking shows that the most important values to students at Victoria University of Wellington are Loyalty, Equality, Helpfulness, and A World at Peace. Past research demonstrated that people who believe in these values always emphasise universal human requirements, and are very interested in understanding, appreciating, tolerating, and protecting the welfare of all close others and people in other settings. Therefore, based 
on the average of students' rankings, we can conclude that they have shown their concern for the welfare of all human beings, even those whose way of life differs from theirs.

We are interested to hear from you why you think students from Victoria University decided on those four values. Please write your own explanation of why students emphasized the four values.

Below you will find a new set of 16 values in alphabetical order. These values have not yet been studied in students before. We would like to know how important each value is as a guiding principle in your life. We are interested in finding out the relative importance of these values to you.

Study the list carefully. Then place a 1 next to the value which is most important to you, place a 2 next to the second value which is second most important, etc. The value which is least important should be ranked 16 .

When you have completed ranking all the values, go back and check over your list. Feel free to make changes. Please take all the time you need to think about this, so that the end result truly represents your values.

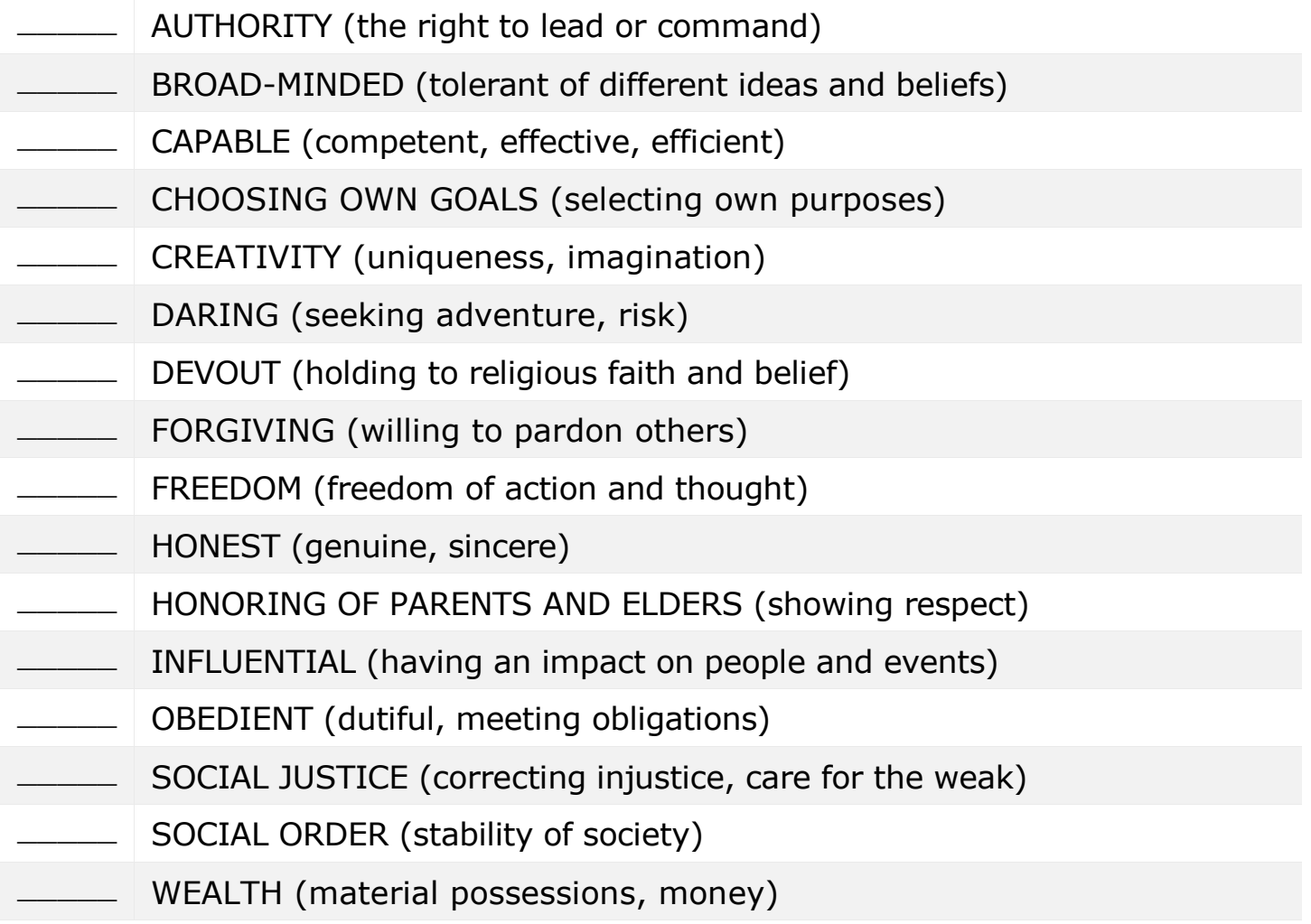




\section{PART 2. General feelings scale (Self-Esteem scale)}

Below is a list of statements dealing with your general feelings about yourself. If you strongly agree, circle SA. If you agree with the statement, circle A. If you disagree, circle D. If you strongly disagree, circle SD.

\begin{tabular}{|c|c|c|c|}
\hline $\begin{array}{c}\text { SA } \\
\text { Strongly agree }\end{array}$ & $\begin{array}{c}\text { A } \\
\text { Agree }\end{array}$ & $\begin{array}{c}\text { D } \\
\text { Disagree }\end{array}$ & $\begin{array}{c}\text { SD } \\
\text { Strongly disagree }\end{array}$ \\
\hline \multicolumn{3}{|c|}{ On the whole, I am satisfied with myself. } & $S A A D S$ \\
\hline \multicolumn{3}{|c|}{ At times, I think I am no good at all. } & $S A$ A D SD \\
\hline \multicolumn{3}{|c|}{ I feel that I have a number of good qualities. } & $S A A D D$ \\
\hline \multicolumn{3}{|c|}{ I am able to do things as well as most other people. } & SA A D SD \\
\hline \multicolumn{3}{|c|}{ I feel I do not have much to be proud of. } & $S A A D S$ \\
\hline \multicolumn{3}{|c|}{ I certainly feel useless at times. } & $S A A D S$ \\
\hline \multicolumn{3}{|c|}{$\begin{array}{l}\text { I feel that I'm a person of worth, at least on an equal plane } \\
\text { with others. }\end{array}$} & $S A$ A D SD \\
\hline \multicolumn{3}{|c|}{ I wish I could have more respect for myself. } & SA A D SD \\
\hline \multicolumn{3}{|c|}{ All in all, I am inclined to feel that I am a failure. } & SA A D SD \\
\hline \multicolumn{3}{|c|}{ I take a positive attitude toward myself. } & $S A A D D$ \\
\hline
\end{tabular}

\section{PART 3. Positive and Negative Affect Schedule}

This scale consists of a number of words that describe different feelings and emotions. Read each item and then mark the appropriate answer in the space next to that word. Indicate to what extent you have felt this way during the past few weeks. Use the following scale to record your answers.

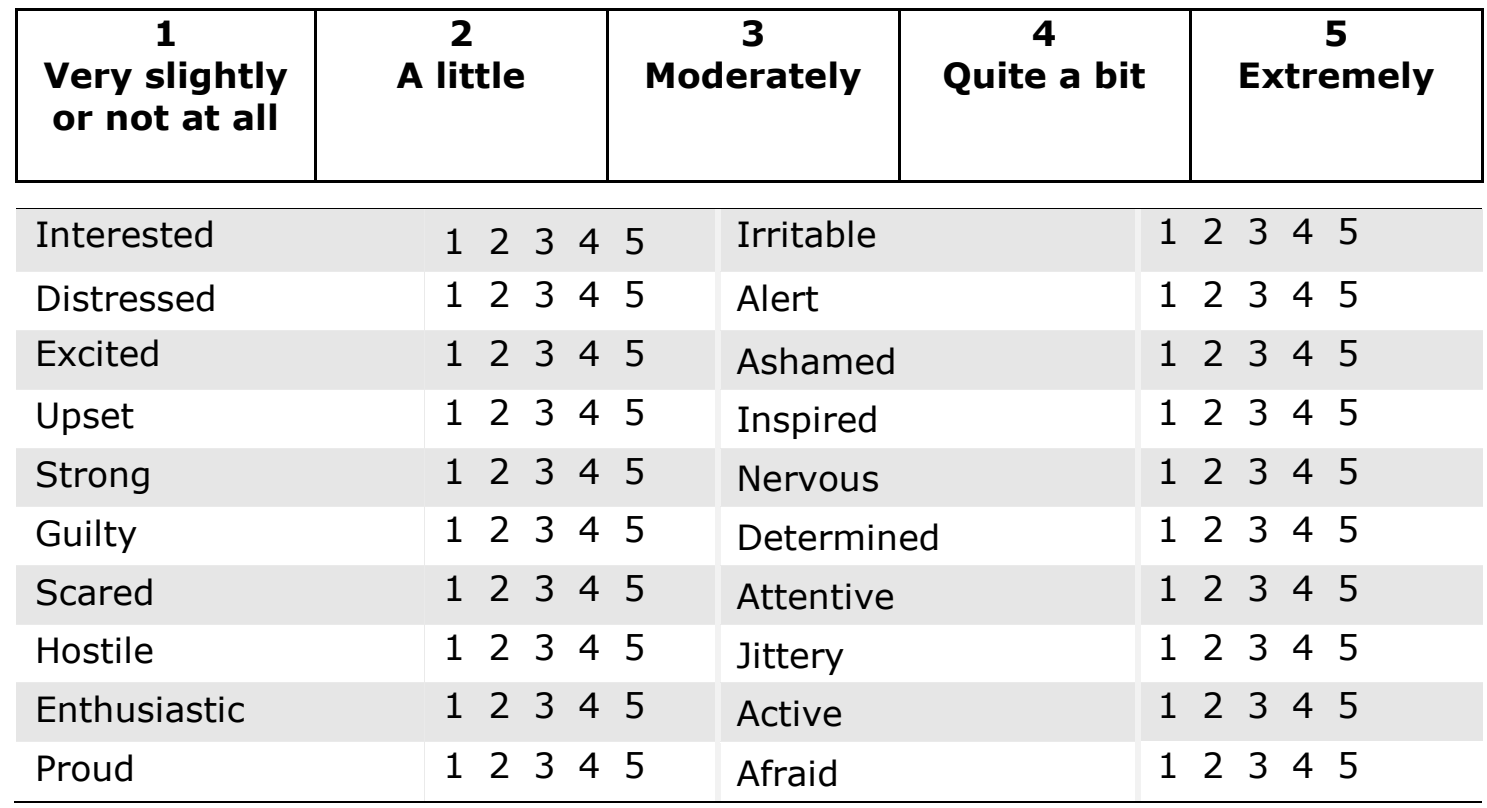




\section{PART 4. General questions about the environment}

Please indicate the extent to which you would be willing to engage in the following behaviours:

\begin{tabular}{|c|c|c|c|c|c|c|}
\hline $\begin{array}{c}0 \\
\text { Not at all } \\
\text { willing } \\
\end{array}$ & $\begin{array}{c}1 \\
\text { A little } \\
\text { willing }\end{array}$ & $\begin{array}{c}2 \\
\begin{array}{c}\text { Moderately } \\
\text { willing }\end{array} \\
\end{array}$ & $\begin{array}{c}3 \\
\text { Very willing }\end{array}$ & & $\begin{array}{r}4 \\
\text { Extreme } \\
\text { willing }\end{array}$ & $\begin{array}{l}\text { iely } \\
\text { ig }\end{array}$ \\
\hline \multicolumn{4}{|c|}{ Collect and recycle used paper. } & 0 & 123 & \\
\hline \multicolumn{4}{|c|}{ Leave the place as clean as it was originally after a picnic. } & 0 & 1232 & \\
\hline \multicolumn{4}{|c|}{ Wait until you have a full load before doing your laundry. } & 0 & 123 & \\
\hline \multicolumn{4}{|c|}{ Buy products in refillable packages. } & 0 & $123<$ & \\
\hline \multicolumn{4}{|c|}{ Ride a bicycle or take public transportation to work or school. } & 0 & $123<$ & \\
\hline \multicolumn{4}{|c|}{ Boycott companies with an unecological background. } & 0 & $123<$ & \\
\hline \multicolumn{4}{|c|}{$\begin{array}{l}\text { Use public transportation or ride a bike, when possible in } \\
\text { nearby areas (around } 30 \mathrm{~km} \text {; around } 20 \text { miles). }\end{array}$} & 0 & $123<$ & 4 \\
\hline \multicolumn{4}{|c|}{$\begin{array}{l}\text { Turn down the heat when I leave my apartment for more } \\
\text { than } 4 \mathrm{~h} \text { during winter. }\end{array}$} & 0 & 1234 & \\
\hline \multicolumn{4}{|c|}{ Be a member of an environmental organization. } & 0 & $123<$ & \\
\hline \multicolumn{4}{|c|}{ Drive on freeways at speeds under 100 k.p.h. (62.5 m.p.h.). } & 0 & 1232 & 4 \\
\hline \multicolumn{4}{|c|}{ Contribute financially to environmental organizations. } & 0 & $123<$ & \\
\hline \multicolumn{4}{|c|}{ Read about environmental issues. } & 0 & $123<$ & \\
\hline \multicolumn{4}{|c|}{ Request an estimate on having solar power installed. } & 0 & 123 & \\
\hline \multicolumn{4}{|c|}{ Buy solar panels to produce energy. } & 0 & 123 & \\
\hline
\end{tabular}

\section{PART 6. Well-Being Index}

Please indicate for each of the five statements which is closest to how you have been feeling over the last two weeks. Notice that higher numbers mean better wellbeing.

Example: If you have felt cheerful and in good spirits more than half of the time during the last two weeks, put a tick in the box with the number 3 .

\begin{tabular}{|c|c|c|c|c|c|}
\hline 0 & 1 & 2 & 3 & 4 \\
At no time & $\begin{array}{c}\text { Some of } \\
\text { the time }\end{array}$ & $\begin{array}{c}\text { Less than } \\
\text { half of the } \\
\text { time }\end{array}$ & $\begin{array}{c}\text { More than } \\
\text { half of the } \\
\text { time }\end{array}$ & $\begin{array}{c}\text { Most of the } \\
\text { time }\end{array}$ & $\begin{array}{c}\text { All of } \\
\text { the time }\end{array}$ \\
\hline
\end{tabular}

Over the last two weeks

I have felt cheerful and in good spirits

I have felt calm and relaxed

I have felt active and vigorous

I woke up feeling fresh and rested

My daily life has been filled with things that interest me $\begin{array}{llllll}0 & 1 & 2 & 3 & 4 & 5\end{array}$

$\begin{array}{llllll}0 & 1 & 2 & 3 & 4 & 5\end{array}$

$\begin{array}{llllll}0 & 1 & 2 & 3 & 4 & 5\end{array}$

$\begin{array}{llllll}0 & 1 & 2 & 3 & 4 & 5\end{array}$

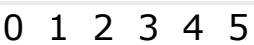




\section{PART 7. Personality traits}

Here are a number of personality traits that may or may not apply to you. Please write a number next to each statement to indicate the extent to which you agree or disagree with that statement. You should rate the extent to which the pair of traits applies to you, even if one characteristic applies more strongly than the other.

\begin{tabular}{|c|c|c|c|c|c|c|}
\hline 1 & 2 & 3 & 4 & 5 & 6 & 7 \\
$\begin{array}{c}\text { Disagree } \\
\text { strongly }\end{array}$ & $\begin{array}{c}\text { Disagree } \\
\text { moderately }\end{array}$ & $\begin{array}{c}\text { Disagree } \\
\text { a little }\end{array}$ & $\begin{array}{c}\text { Neither } \\
\text { agree } \\
\text { nor } \\
\text { disagree }\end{array}$ & $\begin{array}{c}\text { Agree } \\
\text { a } \\
\text { little }\end{array}$ & $\begin{array}{c}\text { Agree } \\
\text { moderately }\end{array}$ & $\begin{array}{c}\text { Agree } \\
\text { strongly }\end{array}$ \\
\hline
\end{tabular}

I see myself as:

Extraverted, enthusiastic

123345567

Reserved, quiet

$\begin{array}{lllllll}1 & 2 & 3 & 4 & 5 & 6 & 7\end{array}$

Sympathetic, warm

$\begin{array}{lllllll}1 & 2 & 3 & 4 & 5 & 6 & 7\end{array}$

Critical, quarrelsome

122345567

Dependable, self-disciplined

$\begin{array}{lllllll}1 & 2 & 3 & 4 & 5 & 6 & 7\end{array}$

Disorganised, careless

1234567

Calm, emotionally stable

1234567

Anxious, easily upset

1234567

Open to new experiences, complex

1234567

Conventional, uncreative

$\begin{array}{lllllll}12 & 34 & 567\end{array}$ 
1. How old are you? years.
2. What is your gender?

1. Female

2. Male
3. Are you a member of any environmental organisation (e.g., Greenpeace)?
1. Yes
2. No

4. Were you born in New Zealand? 5. How long have you been living in New Zealand?
1. Yes
2. No

6. Which ethnic group do you belong to? Please indicate the group you most strongly identify with.
1. New Zealand European (Pākehā)
4. Māori
2. Pacific Nations
5. Asian
3. Indian
6. Other (please specify):

You will be debriefed about this experiment by the end of this semester. However, I'm curious to know your perception of this study. What do you think the purpose of this study was? Did you have any ideas about what kind of results we might be looking at? What did you think we were hoping to learn about? If you are unsure what the purpose is, please state this:

Please write any general comments you would like to make about this questionnaire or the study in general:

\section{Thank you for your time! :)}

Because we are interested in the responses each person makes without the influence of others, we ask that you not discuss this study with your classmates until sometime towards the end of the semester, when all data has been collected and we will send you an email with a debriefing sheet. If you would like to know the results of this study, they will be available at the end of the semester via email upon request. 


\section{Your own list of values}

A VARIED LIFE (filled with challenge, novelty, and change)

A WORLD AT PEACE (free of war and conflict)

AMBITIOUS (hardworking, aspiring)

AN EXCITING LIFE (stimulating experiences)

CURIOUS (interested in everything, exploring)

DETACHMENT (from worldly concerns)

EQUALITY (equal opportunity for all)

HELPFUL (working for the welfare of others)

INDEPENDENT (self-reliant, self-sufficient)

LOYAL (faithful to my friends, group)

MODERATE (avoiding extremes of feeling and action)

POLITENES (courtesy, good manners)

RESPECT FOR TRADITION (preservation of time-honoured customs)

SOCIAL POWER (control over others, dominance)

SOCIAL RECOGNITION (respect, approval by others)

SUCCESSFUL (achieving goals) 


\section{APPENDIX G}

\section{Self-enhancement Values Condition - Experiment 1}

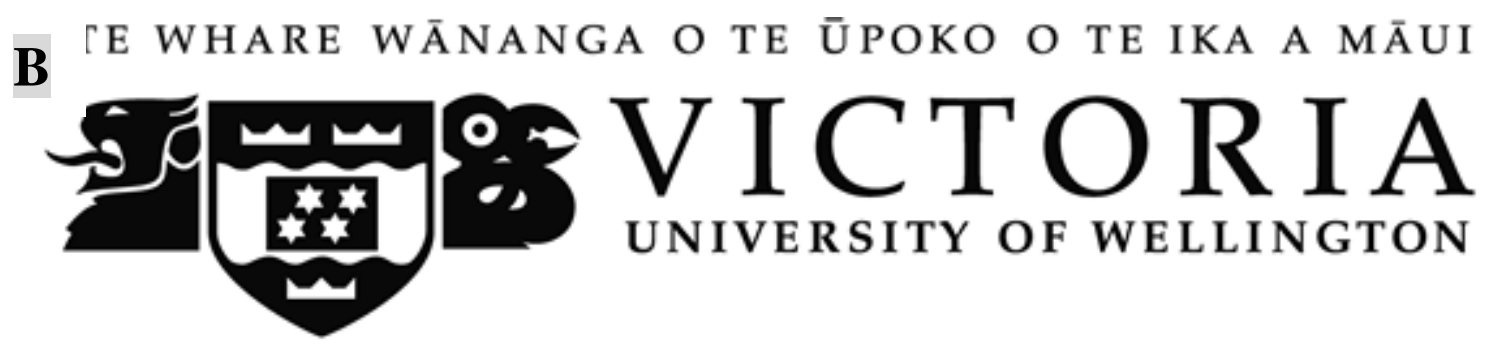

\section{Part 1. Personal values}

Now, I would like to tell you some things we have already found about the value systems of Victoria University students. I am sure that many of you would like to know what they are.

The same value system scale that you answered previously in the mass testing was filled out by 298 students in Psychology 122 . The responses of these students were obtained and averaged together. The table below shows the results.

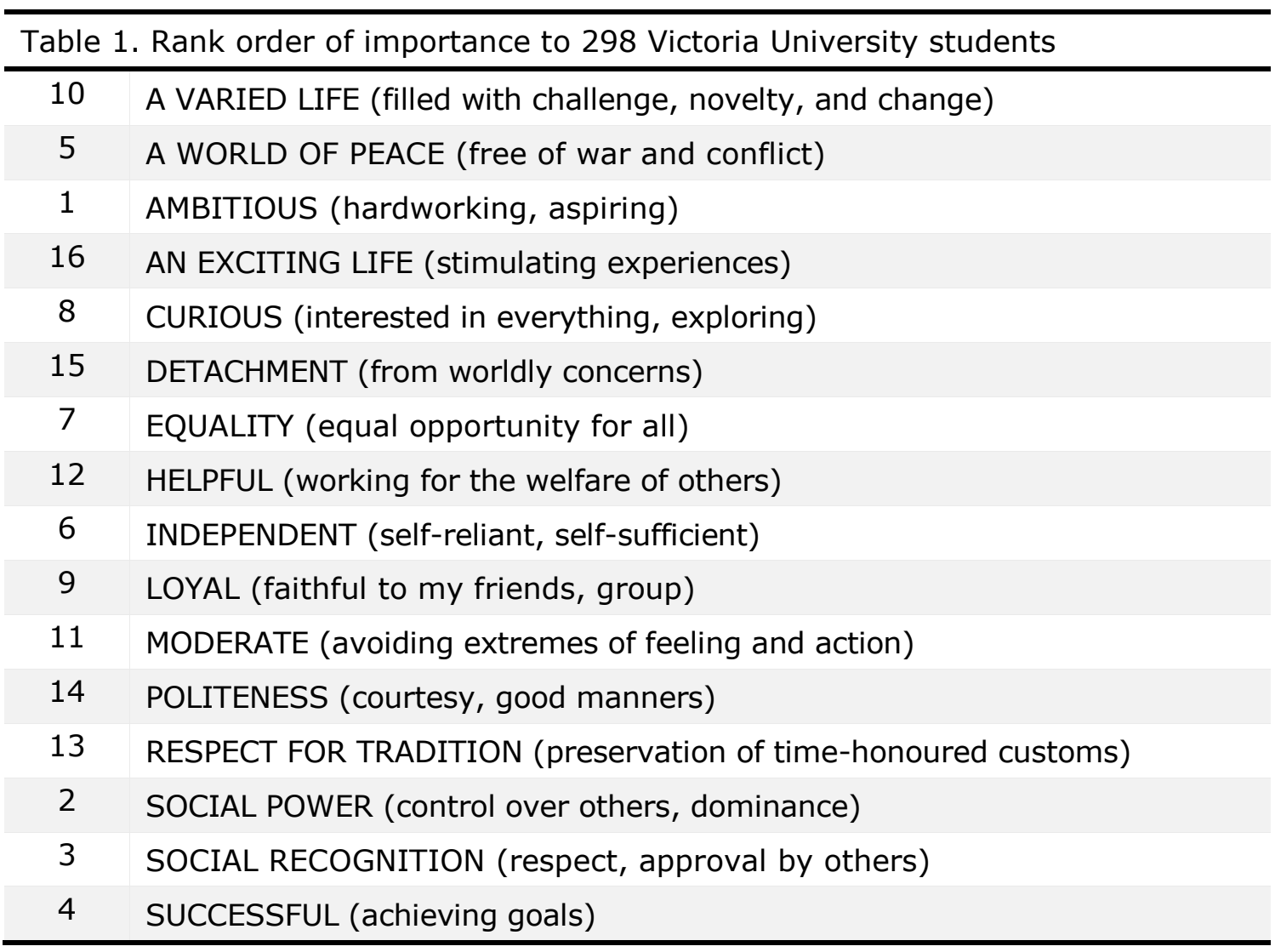

You have your own rankings (from the mass testing) at the end of this questionnaire. Feel free to spend a few minutes comparing your own rankings with those of the 298 students, shown in Table 1. 
Please identify the four most highly ranked values in the Victoria University students ranking and write the names of the values in the predetermined column below.

The four most highly ranked values for Victoria University students were:

1 st

2nd

3rd

4th

Now, do the same task for your own value rankings. Please identify the four most highly ranked values in your own ranking and write the names of the values in the predetermined column below.

The four most highly ranked values for you were:

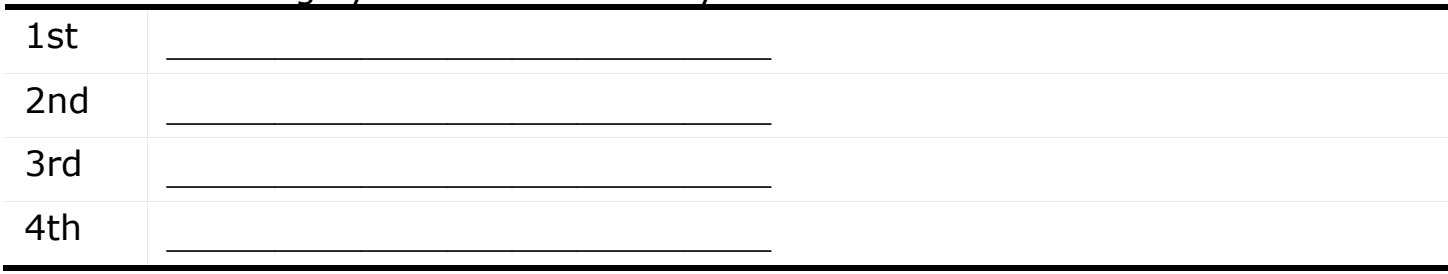

Can you recognize any similarities and differences between your value rankings and the student's value rankings? Please write down your comments.

Below we have an explanation about the first four preferred values of Victoria University students and about the characteristics of people who rank those values as their most preferred values. Please spend some time reading through the following explanation.

The average of the New Zealanders' value ranking shows that the most important values to students at Victoria University of Wellington are Ambition, Social power, Social recognition, and Successfulness. Past research demonstrated that people who believe in these values always emphasise achievement and personal success through demonstrating competence according to social standards and attainment of social status and prestige. Therefore, based on the average of students' rankings, we can conclude that they have shown their concern for active 
demonstration of competence in concrete interaction and attainment of a dominant position within a social system. We are interested to hear from you why you think students from Victoria University decided on those four values. Please write your own explanation of why students emphasized the four values.

Below you will find a new set of 16 values in alphabetical order. These values have not yet been studied in students before. We would like to know how important each value is as a guiding principle in your life. We are interested in finding out the relative importance of these values to you.

Study the list carefully. Then place a 1 next to the value which is most important to you, place a 2 next to the second value which is second most important, etc. The value which is least important should be ranked 16 .

When you have completed ranking all the values, go back and check over your list. Feel free to make changes. Please take all the time you need to think about this, so that the end result truly represents your values.

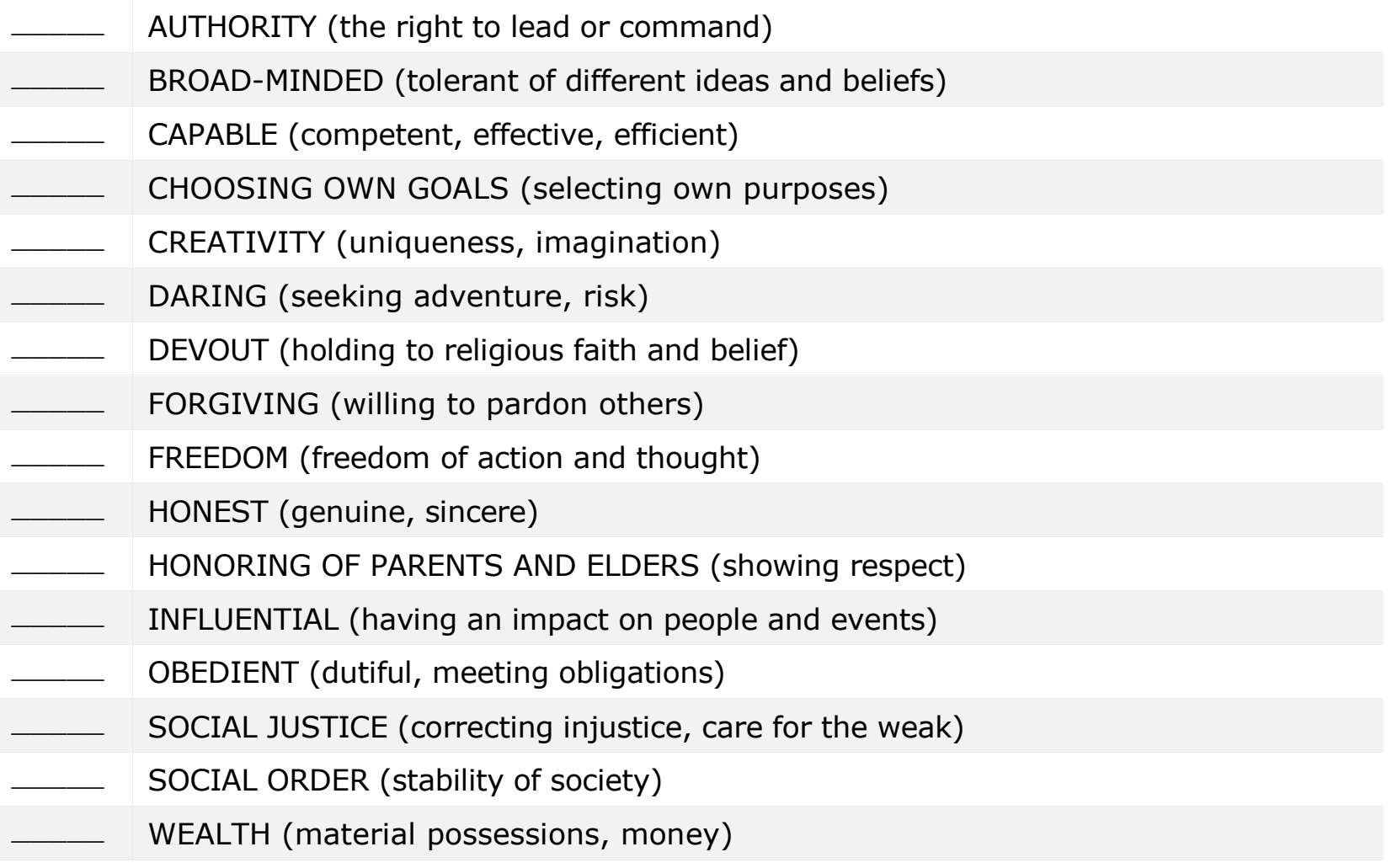




\section{PART 2. General feelings scale (Self-Esteem scale)}

Below is a list of statements dealing with your general feelings about yourself. If you strongly agree, circle SA. If you agree with the statement, circle A. If you disagree, circle D. If you strongly disagree, circle SD.

\begin{tabular}{|c|c|c|c|}
\hline $\begin{array}{c}\text { SA } \\
\text { Strongly agree }\end{array}$ & $\begin{array}{c}\text { A } \\
\text { Agree } \\
\end{array}$ & $\begin{array}{c}\text { D } \\
\text { Disagree }\end{array}$ & $\begin{array}{c}\text { SD } \\
\text { Strongly disagree }\end{array}$ \\
\hline \multicolumn{3}{|c|}{ On the whole, I am satisfied with myself. } & $S A$ A D SD \\
\hline \multicolumn{3}{|c|}{ At times, I think I am no good at all. } & SA A D SD \\
\hline \multicolumn{3}{|c|}{ I feel that I have a number of good qualities. } & SA A D SD \\
\hline \multicolumn{3}{|c|}{ I am able to do things as well as most other people. } & $S A$ A D SD \\
\hline \multicolumn{3}{|c|}{ I feel I do not have much to be proud of. } & SA A D SD \\
\hline \multicolumn{3}{|c|}{ I certainly feel useless at times. } & SA A D SD \\
\hline \multicolumn{3}{|c|}{$\begin{array}{l}\text { I feel that I'm a person of worth, at least on an equal plane } \\
\text { with others. }\end{array}$} & SA A D SD \\
\hline \multicolumn{3}{|c|}{ I wish I could have more respect for myself. } & SA A D SD \\
\hline \multicolumn{3}{|c|}{ All in all, I am inclined to feel that I am a failure. } & SA A D SD \\
\hline \multicolumn{3}{|c|}{ I take a positive attitude toward myself. } & SA A D SD \\
\hline
\end{tabular}

\section{PART 3. Positive and Negative Affect Schedule}

This scale consists of a number of words that describe different feelings and emotions. Read each item and then mark the appropriate answer in the space next to that word. Indicate to what extent you have felt this way during the past few weeks. Use the following scale to record your answers.

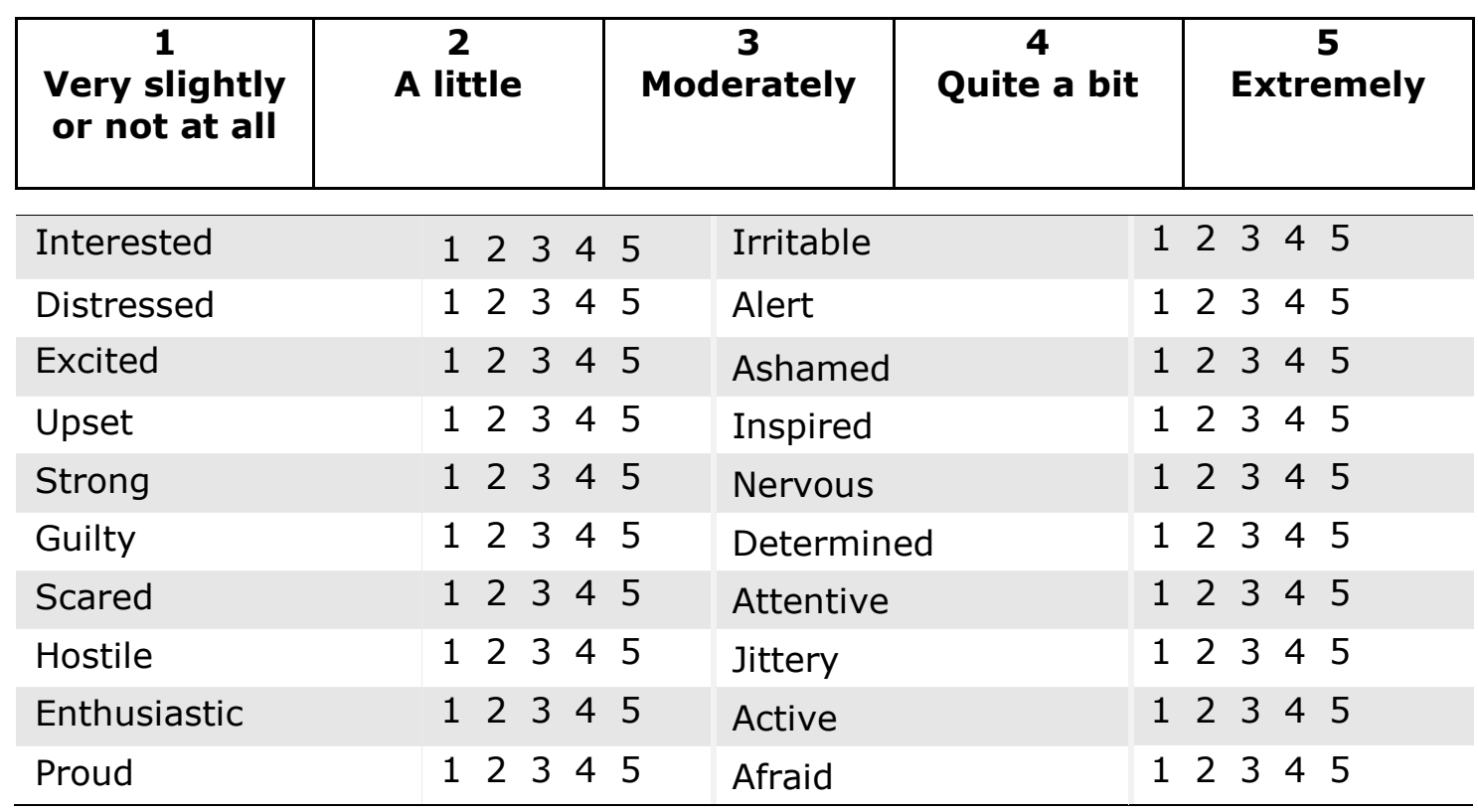




\section{PART 4. General questions about the environment}

Please indicate the extent to which you would be willing to engage in the following behaviours:

\begin{tabular}{|c|c|c|c|c|c|c|}
\hline $\begin{array}{c}0 \\
\text { Not at all } \\
\text { willing } \\
\end{array}$ & $\begin{array}{c}1 \\
\text { A little } \\
\text { willing }\end{array}$ & $\begin{array}{c}2 \\
\begin{array}{c}\text { Moderately } \\
\text { willing }\end{array} \\
\end{array}$ & $\begin{array}{c}3 \\
\text { Very willing }\end{array}$ & & $\begin{array}{r}4 \\
\text { Extreme } \\
\text { willing }\end{array}$ & $\begin{array}{l}\text { iely } \\
\text { ig }\end{array}$ \\
\hline \multicolumn{4}{|c|}{ Collect and recycle used paper. } & 0 & 123 & \\
\hline \multicolumn{4}{|c|}{ Leave the place as clean as it was originally after a picnic. } & 0 & 1232 & \\
\hline \multicolumn{4}{|c|}{ Wait until you have a full load before doing your laundry. } & 0 & 123 & \\
\hline \multicolumn{4}{|c|}{ Buy products in refillable packages. } & 0 & 1232 & \\
\hline \multicolumn{4}{|c|}{ Ride a bicycle or take public transportation to work or school. } & 0 & $123<$ & \\
\hline \multicolumn{4}{|c|}{ Boycott companies with an unecological background. } & 0 & $123<$ & \\
\hline \multicolumn{4}{|c|}{$\begin{array}{l}\text { Use public transportation or ride a bike, when possible in } \\
\text { nearby areas (around } 30 \mathrm{~km} \text {; around } 20 \text { miles). }\end{array}$} & 0 & $123<$ & 4 \\
\hline \multicolumn{4}{|c|}{$\begin{array}{l}\text { Turn down the heat when I leave my apartment for more } \\
\text { than } 4 \mathrm{~h} \text { during winter. }\end{array}$} & 0 & 1234 & \\
\hline \multicolumn{4}{|c|}{ Be a member of an environmental organization. } & 0 & $123<$ & \\
\hline \multicolumn{4}{|c|}{ Drive on freeways at speeds under 100 k.p.h. (62.5 m.p.h.). } & 0 & 1232 & 4 \\
\hline \multicolumn{4}{|c|}{ Contribute financially to environmental organizations. } & 0 & $123<$ & \\
\hline \multicolumn{4}{|c|}{ Read about environmental issues. } & 0 & $123<$ & \\
\hline \multicolumn{4}{|c|}{ Request an estimate on having solar power installed. } & 0 & 123 & \\
\hline \multicolumn{4}{|c|}{ Buy solar panels to produce energy. } & 0 & 123 & \\
\hline
\end{tabular}

\section{PART 6. Well-Being Index}

Please indicate for each of the five statements which is closest to how you have been feeling over the last two weeks. Notice that higher numbers mean better wellbeing.

Example: If you have felt cheerful and in good spirits more than half of the time during the last two weeks, put a tick in the box with the number 3 .

\begin{tabular}{|c|c|c|c|c|c|}
\hline 0 & 1 & 2 & 3 & 4 \\
At no time & $\begin{array}{c}\text { Some of } \\
\text { the time }\end{array}$ & $\begin{array}{c}\text { Less than } \\
\text { half of the } \\
\text { time }\end{array}$ & $\begin{array}{c}\text { More than } \\
\text { half of the } \\
\text { time }\end{array}$ & $\begin{array}{c}\text { Most of the } \\
\text { time }\end{array}$ & $\begin{array}{c}\text { All of } \\
\text { the time }\end{array}$ \\
\hline
\end{tabular}

Over the last two weeks

I have felt cheerful and in good spirits

I have felt calm and relaxed

I have felt active and vigorous

I woke up feeling fresh and rested

My daily life has been filled with things that interest me
$\begin{array}{llllll}0 & 1 & 2 & 3 & 4 & 5\end{array}$
$\begin{array}{llllll}0 & 1 & 2 & 3 & 4 & 5\end{array}$
$\begin{array}{llllll}0 & 1 & 2 & 3 & 4 & 5\end{array}$
$\begin{array}{llllll}0 & 1 & 2 & 3 & 4 & 5\end{array}$
$\begin{array}{llllll}0 & 1 & 2 & 3 & 4 & 5\end{array}$ 


\section{PART 7. Personality traits}

Here are a number of personality traits that may or may not apply to you. Please write a number next to each statement to indicate the extent to which you agree or disagree with that statement. You should rate the extent to which the pair of traits applies to you, even if one characteristic applies more strongly than the other.

\begin{tabular}{|c|c|c|c|c|c|c|}
\hline 1 & 2 & 3 & 4 & 5 & 6 & 7 \\
$\begin{array}{c}\text { Disagree } \\
\text { strongly }\end{array}$ & $\begin{array}{c}\text { Disagree } \\
\text { moderately }\end{array}$ & $\begin{array}{c}\text { Disagree } \\
\text { a little }\end{array}$ & $\begin{array}{c}\text { Neither } \\
\text { agree } \\
\text { nor } \\
\text { disagree }\end{array}$ & $\begin{array}{c}\text { Agree } \\
\text { a } \\
\text { little }\end{array}$ & $\begin{array}{c}\text { Agree } \\
\text { moderately }\end{array}$ & $\begin{array}{c}\text { Agree } \\
\text { strongly }\end{array}$ \\
\hline
\end{tabular}

I see myself as:

Extraverted, enthusiastic

123345567

Reserved, quiet

$\begin{array}{lllllll}1 & 2 & 3 & 4 & 5 & 6 & 7\end{array}$

Sympathetic, warm

$\begin{array}{lllllll}1 & 2 & 3 & 4 & 5 & 6 & 7\end{array}$

Critical, quarrelsome

122345567

Dependable, self-disciplined

$\begin{array}{lllllll}1 & 2 & 3 & 4 & 5 & 6 & 7\end{array}$

Disorganised, careless

1234567

Calm, emotionally stable

1234567

Anxious, easily upset

1234567

Open to new experiences, complex

1234567

Conventional, uncreative

$\begin{array}{lllllll}12 & 34 & 567\end{array}$ 
1. How old are you? years.
2. What is your gender?

1. Female

2. Male
3. Are you a member of any environmental organisation (e.g., Greenpeace)?
1. Yes
2. No

4. Were you born in New Zealand? 5. How long have you been living in New Zealand?
1. Yes
2. No

6. Which ethnic group do you belong to? Please indicate the group you most strongly identify with.
1. New Zealand European (Pākehā)
4. Māori
2. Pacific Nations
5. Asian
3. Indian
6. Other (please specify):

You will be debriefed about this experiment by the end of this semester. However, I'm curious to know your perception of this study. What do you think the purpose of this study was? Did you have any ideas about what kind of results we might be looking at? What did you think we were hoping to learn about? If you are unsure what the purpose is, please state this:

Please write any general comments you would like to make about this questionnaire or the study in general:

\section{Thank you for your time! :)}

Because we are interested in the responses each person makes without the influence of others, we ask that you not discuss this study with your classmates until sometime towards the end of the semester, when all data has been collected and we will send you an email with a debriefing sheet. If you would like to know the results of this study, they will be available at the end of the semester via email upon request. 


\section{Your own list of values}

A VARIED LIFE (filled with challenge, novelty, and change)

A WORLD AT PEACE (free of war and conflict)

AMBITIOUS (hardworking, aspiring)

AN EXCITING LIFE (stimulating experiences)

CURIOUS (interested in everything, exploring)

DETACHMENT (from worldly concerns)

EQUALITY (equal opportunity for all)

HELPFUL (working for the welfare of others)

INDEPENDENT (self-reliant, self-sufficient)

LOYAL (faithful to my friends, group)

MODERATE (avoiding extremes of feeling and action)

POLITENES (courtesy, good manners)

RESPECT FOR TRADITION (preservation of time-honoured customs)

SOCIAL POWER (control over others, dominance)

SOCIAL RECOGNITION (respect, approval by others)

SUCCESSFUL (achieving goals) 


\section{Control Condition - Experiment 1}

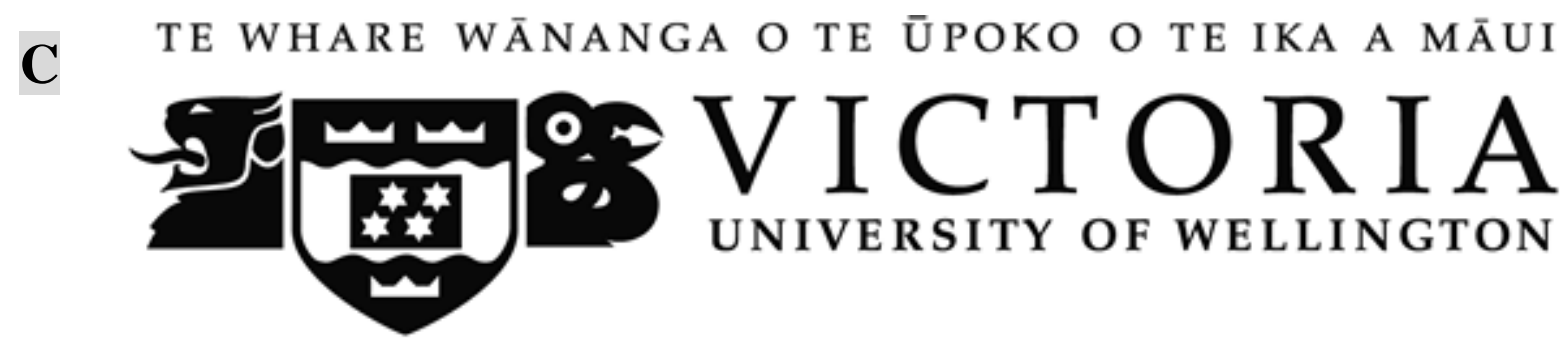

\section{PART 1. Dessert preferences (Part 1)}

Below is a list of 16 desserts in alphabetical order. We are interested in finding out the relative preferences of these desserts to you.

Study the list carefully. Then place a 1 next to the dessert which you prefer most, place a 2 next to the second dessert which is second most preferred, etc. The dessert which is least preferred should be ranked 16 .

When you completed ranking all the options, go back and check over your list. Feel free to make changes. Please take all the time you need to think about this, so that the end result truly represents your preferences.

\begin{tabular}{|c|}
\hline APPLE CRUMBLE \\
\hline BANANA BREAD \\
\hline BLUEBERRY MUFFIN \\
\hline BUTTERSCOTCH PUDDING \\
\hline CHOCOLATE BROWNIE \\
\hline CHOCOLATE MOUSSE \\
\hline CUSTARD WITH FRESH SEASONAL FRUITS \\
\hline FRUIT CAKE \\
\hline LAMINGTON WITH CREAM \\
\hline LEMON TART \\
\hline MACADAMIA CARAMEL \\
\hline ORANGE ALMOND CAKE \\
\hline PAVLOVA \\
\hline PURÉED RHUBARB AND APPLE TURNOVER \\
\hline RASPBERRY CREAM SPONGE \\
\hline TRIFLE \\
\hline
\end{tabular}

Now, I would like to tell you some things we have already found about the desserts preferences of Victoria University Students. I am sure that many of you would like to know what they are. 
The same dessert preferences scale that you answered previously was filled out by 298 students in Psychology. The responses of these students were obtained and averaged together. The table below shows the results.

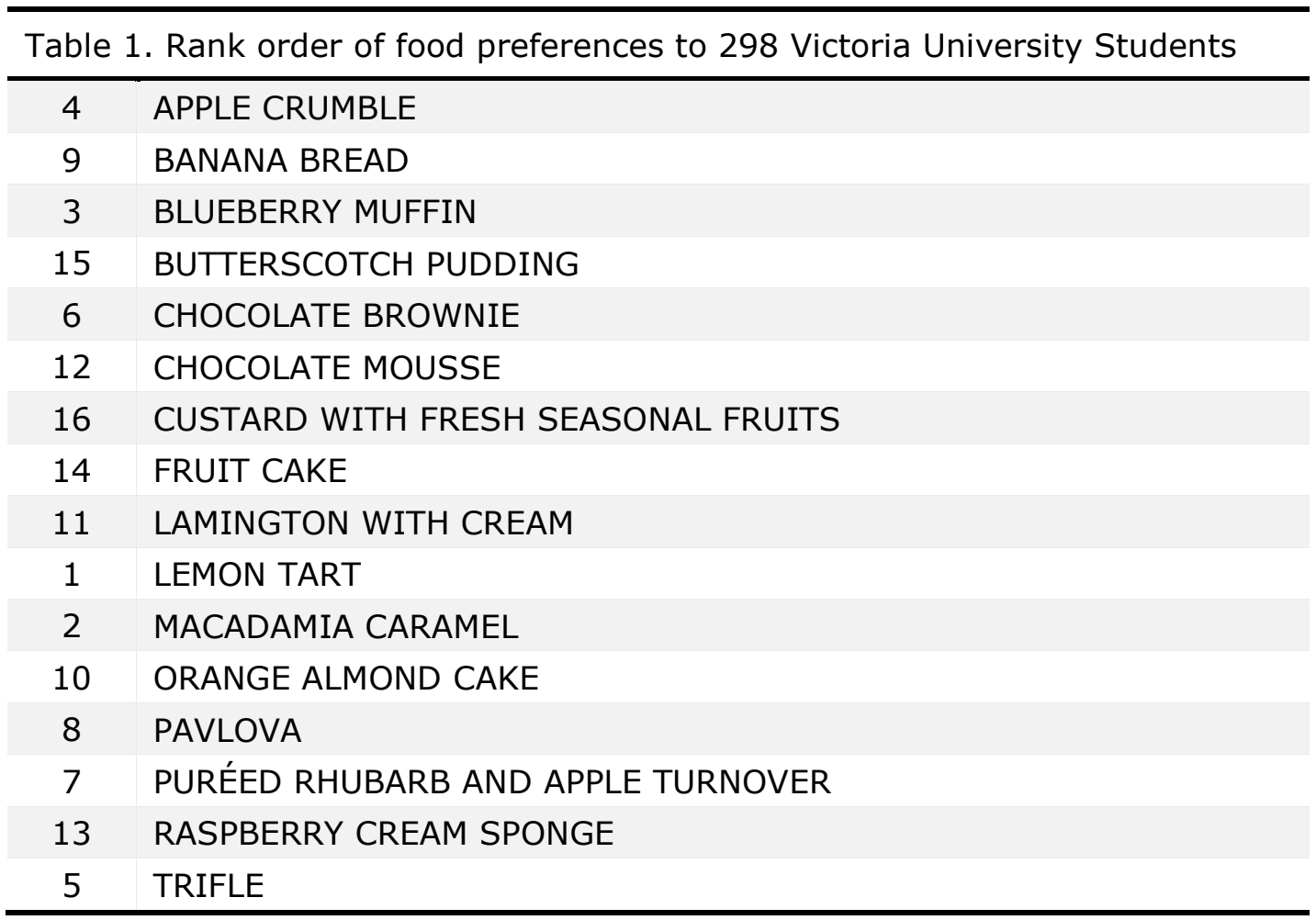

You have your own rankings on the preceding page. Feel free to spend a few minutes comparing your own rankings with those of the 298 students, shown in Table 1.

Please identify the four most highly ranked dessert preferences in the Victoria University students ranking and write the names of the dishes in a predetermined column below.

The four most highly ranked desserts for Victoria University students were:

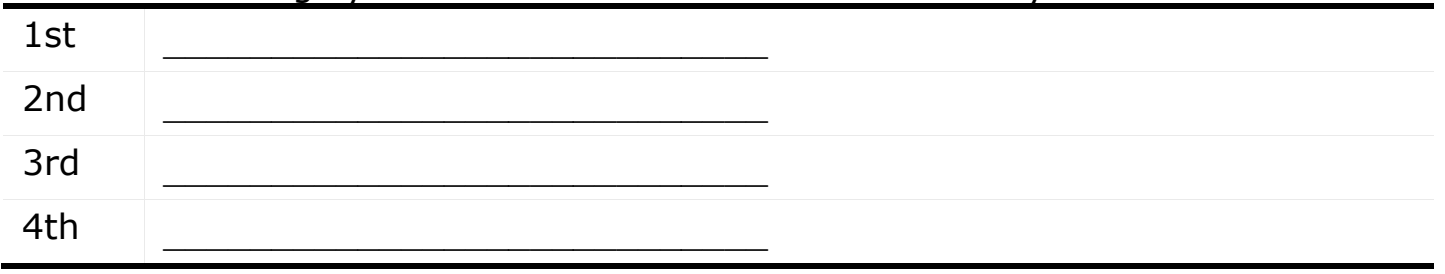

Now, do the same task for your own dessert preferences rankings. Please identify the four most highly ranked dessert preferences in your own ranking and write the names of the desserts in a predetermined column below.

The four most highly ranked desserts for you were:

1 st

2nd 
Can you recognize any similarities and differences between your dessert preferences rankings and the student's rankings? Please write down your comments.

Below we have an explanation about the first four preferred options of desserts for Victoria University students and about the characteristics of people who rank those desserts as their most preferred options. Please spend some time reading through the following explanation.

The average of the students' evaluations of what would be the best options for dessert showed lemon tart, macadamia caramel, blueberry muffin and apple crumble as the four favourites. Past research demonstrated that people who decide on flavours of food base their choices on personal preferences and dietary requirements. Therefore, based on the average of students' rankings, we can conclude that they have shown their preferences while taking into account their favourite desserts in their country.

We are interested to hear from you why you think students from Victoria University decided on those four options for desserts. Please write your own explanation of why students emphasized the four preferences.

\section{PART 2. Dessert preferences (Part 2)}

Below you will find a new set of 16 desserts in alphabetical order. These desserts have not yet been studied in students before. We are interested in finding out the relative preferences of these desserts to you.

Study the list carefully. Then place a 1 next to the dessert which you prefer most, place a 2 next to the second dessert which is second most preferred, etc. The dessert which is least preferred should be ranked 16 . 
When you have completed ranking all the options, go back and check over your list. Feel free to make changes. Please take all the time you need to think about this, so that the end result truly represents your preferences.

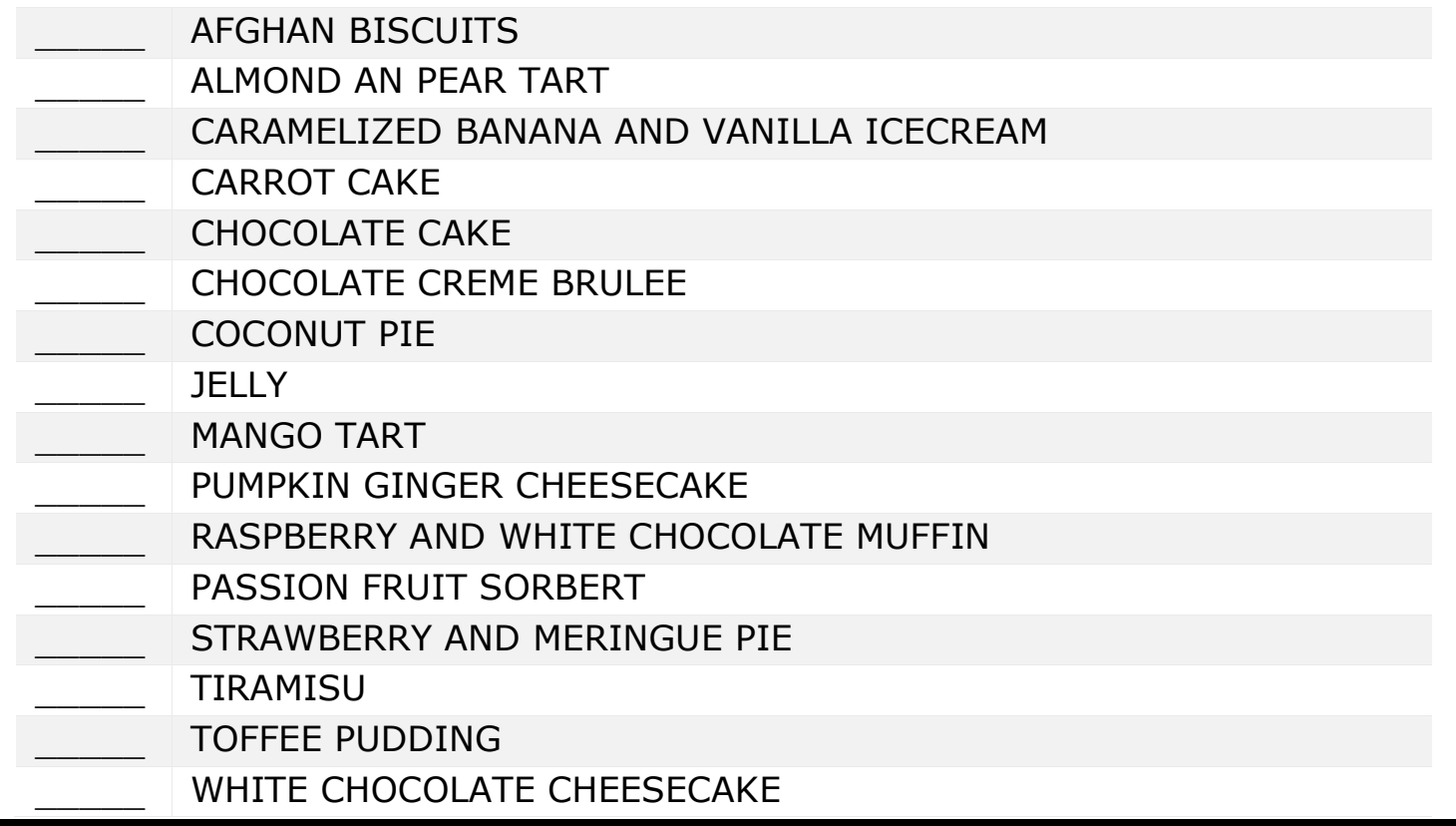

\section{PART 3. Personal values}

Below you will find a new set of 16 values in alphabetical order. These values have not yet been studied in students before. We would like to know how important each value is as a guiding principle in your life. We are interested in finding out the relative importance of these values to you.

Study the list carefully. Then place a 1 next to the value which is most important to you, place a 2 next to the second value which is second most important, etc. The value which is least important should be ranked 16 .

When you have completed ranking all the values, go back and check over your list. Feel free to make changes. Please take all the time you need to think about this, so that the end result truly represents your values.

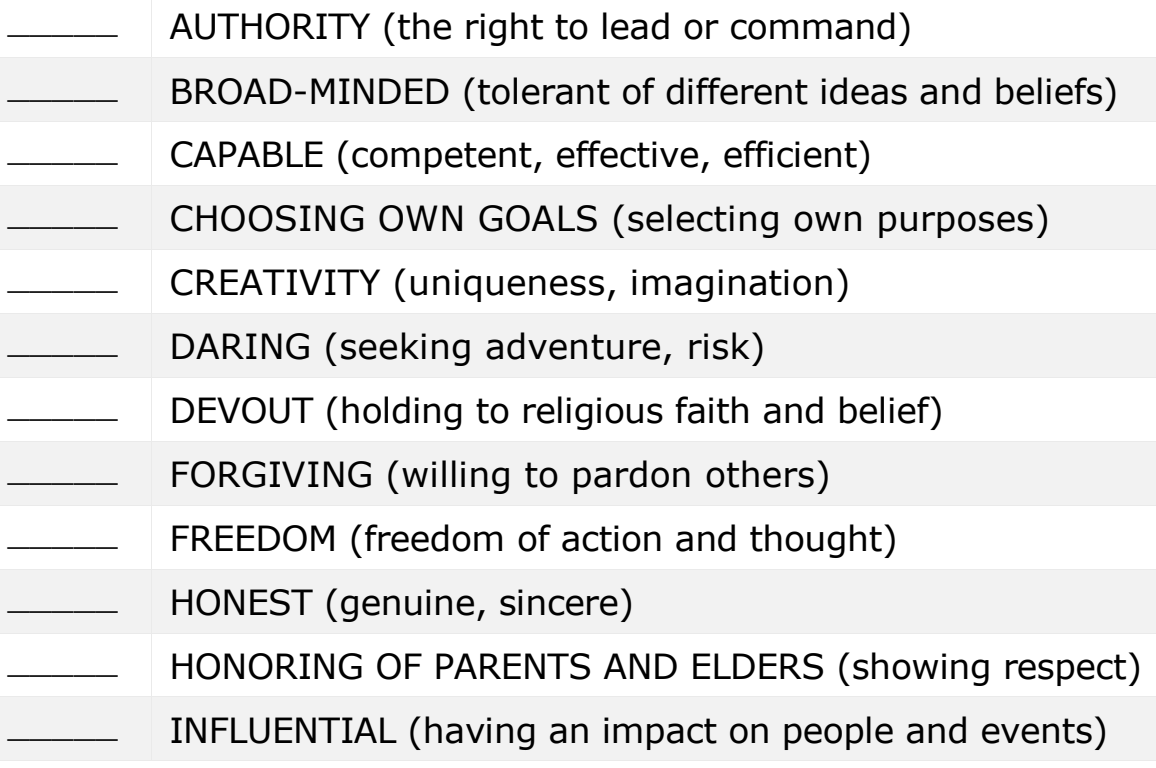


OBEDIENT (dutiful, meeting obligations)

SOCIAL JUSTICE (correcting injustice, care for the weak)

SOCIAL ORDER (stability of society)

WEALTH (material possessions, money)

\section{PART 2. General feelings scale (Self-Esteem scale)}

Below is a list of statements dealing with your general feelings about yourself. If you strongly agree, circle SA. If you agree with the statement, circle A. If you disagree, circle D. If you strongly disagree, circle SD.

\begin{tabular}{|c|c|c|c|}
\hline $\begin{array}{c}\text { SA } \\
\text { Strongly agree }\end{array}$ & $\begin{array}{c}\text { A } \\
\text { Agree }\end{array}$ & $\begin{array}{c}\text { D } \\
\text { Disagree }\end{array}$ & $\begin{array}{c}\text { SD } \\
\text { Strongly disagree }\end{array}$ \\
\hline \multicolumn{3}{|c|}{ On the whole, I am satisfied with myself. } & $S A$ A D SD \\
\hline \multicolumn{3}{|c|}{ At times, I think I am no good at all. } & SA A D SD \\
\hline \multicolumn{3}{|c|}{ I feel that I have a number of good qualities. } & SA A D SD \\
\hline \multicolumn{3}{|c|}{ I am able to do things as well as most other people. } & $S A$ A $D$ SD \\
\hline \multicolumn{3}{|c|}{ I feel I do not have much to be proud of. } & $S A$ A $D$ SD \\
\hline \multicolumn{3}{|c|}{ I certainly feel useless at times. } & $S A$ A D SD \\
\hline \multicolumn{3}{|c|}{$\begin{array}{l}\text { I feel that I'm a person of worth, at least on an equal plane } \\
\text { with others. }\end{array}$} & SA A D SD \\
\hline \multicolumn{3}{|c|}{ I wish I could have more respect for myself. } & $S A$ A D SD \\
\hline \multicolumn{3}{|c|}{ All in all, I am inclined to feel that I am a failure. } & SA A D SD \\
\hline \multicolumn{3}{|c|}{ I take a positive attitude toward myself. } & $S A$ A $D$ SD \\
\hline
\end{tabular}

\section{PART 3. Positive and Negative Affect Schedule}

This scale consists of a number of words that describe different feelings and emotions. Read each item and then mark the appropriate answer in the space next to that word. Indicate to what extent you have felt this way during the past few weeks. Use the following scale to record your answers.

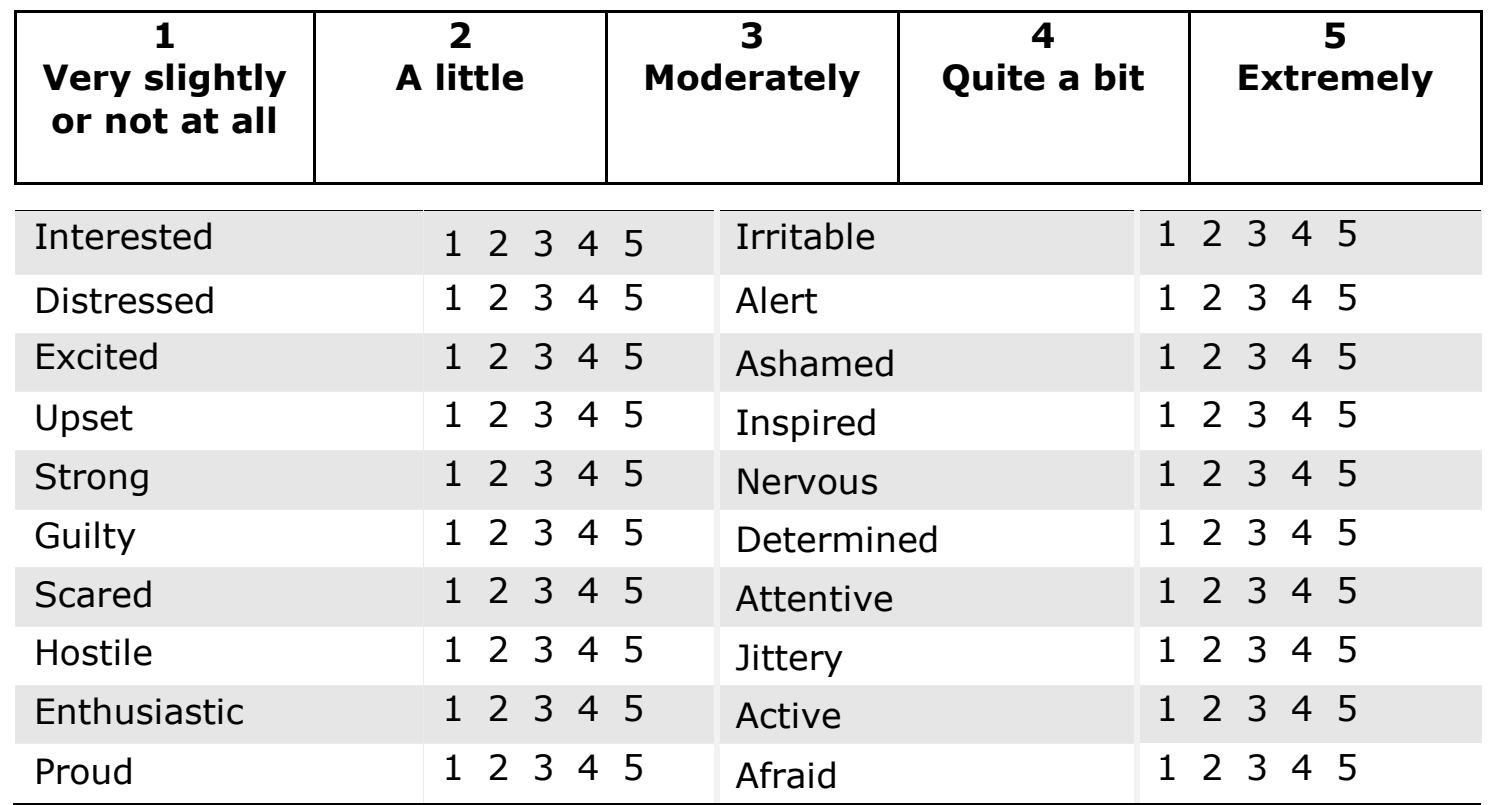


Please indicate the extent to which you would be willing to engage in the following behaviours:

\begin{tabular}{|c|c|c|c|c|c|c|}
\hline $\begin{array}{c}0 \\
\text { Not at all } \\
\text { willing }\end{array}$ & $\begin{array}{c}1 \\
\text { A little } \\
\text { willing } \\
\end{array}$ & $\begin{array}{c}2 \\
\begin{array}{c}\text { Moderately } \\
\text { willing }\end{array} \\
\end{array}$ & $\begin{array}{c}3 \\
\text { Very willing }\end{array}$ & & $\begin{array}{c}4 \\
\text { Extrem } \\
\text { willin } \\
\end{array}$ & \\
\hline \multicolumn{4}{|c|}{ Collect and recycle used paper. } & 0 & 123 & \\
\hline \multicolumn{4}{|c|}{ Leave the place as clean as it was originally after a picnic. } & 0 & 123 & \\
\hline \multicolumn{4}{|c|}{ Wait until you have a full load before doing your laundry. } & 0 & 123 & \\
\hline \multicolumn{4}{|c|}{ Buy products in refillable packages. } & 0 & 123 & \\
\hline \multicolumn{4}{|c|}{ Ride a bicycle or take public transportation to work or school. } & 0 & 123 & \\
\hline \multicolumn{4}{|c|}{ Boycott companies with an unecological background. } & 0 & 123 & \\
\hline \multicolumn{4}{|c|}{$\begin{array}{l}\text { Use public transportation or ride a bike, when possible in } \\
\text { nearby areas (around } 30 \mathrm{~km} \text {; around } 20 \text { miles). }\end{array}$} & 0 & 123 & \\
\hline \multicolumn{4}{|c|}{$\begin{array}{l}\text { Turn down the heat when I leave my apartment for more } \\
\text { than } 4 \mathrm{~h} \text { during winter. }\end{array}$} & 0 & 123 & \\
\hline \multicolumn{4}{|c|}{ Be a member of an environmental organization. } & 0 & 123 & \\
\hline \multicolumn{4}{|c|}{ Drive on freeways at speeds under 100 k.p.h. (62.5 m.p.h.). } & 0 & 123 & \\
\hline \multicolumn{4}{|c|}{ Contribute financially to environmental organizations. } & 0 & 123 & \\
\hline \multicolumn{4}{|c|}{ Read about environmental issues. } & 0 & 123 & \\
\hline \multicolumn{4}{|c|}{ Request an estimate on having solar power installed. } & 0 & 123 & \\
\hline \multicolumn{4}{|c|}{ Buy solar panels to produce energy. } & 0 & 123 & \\
\hline
\end{tabular}

\section{PART 6. Well-Being Index}

Please indicate for each of the five statements which is closest to how you have been feeling over the last two weeks. Notice that higher numbers mean better wellbeing.

Example: If you have felt cheerful and in good spirits more than half of the time during the last two weeks, put a tick in the box with the number 3.

\begin{tabular}{|c|c|c|c|c|c|}
\hline $\mathbf{0}$ & 1 & 2 & 3 & 4 \\
At no time & $\begin{array}{c}\text { Some of } \\
\text { the time }\end{array}$ & $\begin{array}{c}\text { Less than } \\
\text { half of the } \\
\text { time }\end{array}$ & $\begin{array}{c}\text { More than } \\
\text { half of the } \\
\text { time }\end{array}$ & $\begin{array}{c}\text { Most of the } \\
\text { time }\end{array}$ & $\begin{array}{c}\text { All of } \\
\text { the time }\end{array}$ \\
\hline
\end{tabular}

Over the last two weeks

I have felt cheerful and in good spirits

I have felt calm and relaxed

I have felt active and vigorous

$\begin{array}{llllll}0 & 1 & 2 & 3 & 4 & 5\end{array}$

I woke up feeling fresh and rested

$\begin{array}{llllll}0 & 1 & 2 & 3 & 4 & 5\end{array}$

My daily life has been filled with things that interest me

$\begin{array}{llllll}0 & 1 & 2 & 3 & 4 & 5\end{array}$ 


\section{PART 7. Personality traits}

Here are a number of personality traits that may or may not apply to you. Please write a number next to each statement to indicate the extent to which you agree or disagree with that statement. You should rate the extent to which the pair of traits applies to you, even if one characteristic applies more strongly than the other.

\begin{tabular}{|c|c|c|c|c|c|c|}
\hline 1 & 2 & 3 & 4 & 5 & 6 & 7 \\
$\begin{array}{c}\text { Disagree } \\
\text { strongly }\end{array}$ & $\begin{array}{c}\text { Disagree } \\
\text { moderately }\end{array}$ & $\begin{array}{c}\text { Disagree } \\
\text { a little }\end{array}$ & $\begin{array}{c}\text { Neither } \\
\text { agree } \\
\text { nor } \\
\text { disagree }\end{array}$ & $\begin{array}{c}\text { Agree } \\
\text { a } \\
\text { little }\end{array}$ & $\begin{array}{c}\text { Agree } \\
\text { moderately }\end{array}$ & $\begin{array}{c}\text { Agree } \\
\text { strongly }\end{array}$ \\
\hline
\end{tabular}

I see myself as:

Extraverted, enthusiastic

123345567

Reserved, quiet

$\begin{array}{lllllll}1 & 2 & 3 & 4 & 5 & 6 & 7\end{array}$

Sympathetic, warm

$\begin{array}{lllllll}1 & 2 & 3 & 4 & 5 & 6 & 7\end{array}$

Critical, quarrelsome

122345567

Dependable, self-disciplined

$\begin{array}{lllllll}1 & 2 & 3 & 4 & 5 & 6 & 7\end{array}$

Disorganised, careless

1234567

Calm, emotionally stable

1234567

Anxious, easily upset

1234567

Open to new experiences, complex

1234567

Conventional, uncreative

$\begin{array}{lllllll}12 & 34 & 567\end{array}$ 
1. How old are you? years.
2. What is your gender?

1. Female

2. Male
3. Are you a member of any environmental organisation (e.g., Greenpeace)?
1. Yes
2. No

4. Were you born in New Zealand? 5. How long have you been living in New Zealand?
1. Yes
2. No

6. Which ethnic group do you belong to? Please indicate the group you most strongly identify with.
1. New Zealand European (Pākehā)
4. Māori
2. Pacific Nations
5. Asian
3. Indian
6. Other (please specify):

You will be debriefed about this experiment by the end of this semester. However, I'm curious to know your perception of this study. What do you think the purpose of this study was? Did you have any ideas about what kind of results we might be looking at? What did you think we were hoping to learn about? If you are unsure what the purpose is, please state this:

Please write any general comments you would like to make about this questionnaire or the study in general:

\section{Thank you for your time! :)}

Because we are interested in the responses each person makes without the influence of others, we ask that you not discuss this study with your classmates until sometime towards the end of the semester, when all data has been collected and we will send you an email with a debriefing sheet. If you would like to know the results of this study, they will be available at the end of the semester via email upon request. 


\section{Your own list of values}

A VARIED LIFE (filled with challenge, novelty, and change)

A WORLD AT PEACE (free of war and conflict)

AMBITIOUS (hardworking, aspiring)

AN EXCITING LIFE (stimulating experiences)

CURIOUS (interested in everything, exploring)

DETACHMENT (from worldly concerns)

EQUALITY (equal opportunity for all)

HELPFUL (working for the welfare of others)

INDEPENDENT (self-reliant, self-sufficient)

LOYAL (faithful to my friends, group)

MODERATE (avoiding extremes of feeling and action)

POLITENES (courtesy, good manners)

RESPECT FOR TRADITION (preservation of time-honoured customs)

SOCIAL POWER (control over others, dominance)

SOCIAL RECOGNITION (respect, approval by others)

SUCCESSFUL (achieving goals) 


\section{Email and Debriefing Sheet for Experiment 1}

\section{Email}

Dear student,

Thank you for taking part in the mass testing and experiment 459 last semester. Please find attached the debriefing statement for the respective experiment.

Thanks again and please do not hesitate to contact me if you have any questions.

Kindest regards,

Pollyane Diniz

$\mathrm{PhD}$ candidate

Centre for Applied Cross-Cultural Research

School of Psychology

Faculty of Science

e-mail: Pollyane.Diniz@vuw.ac.nz 


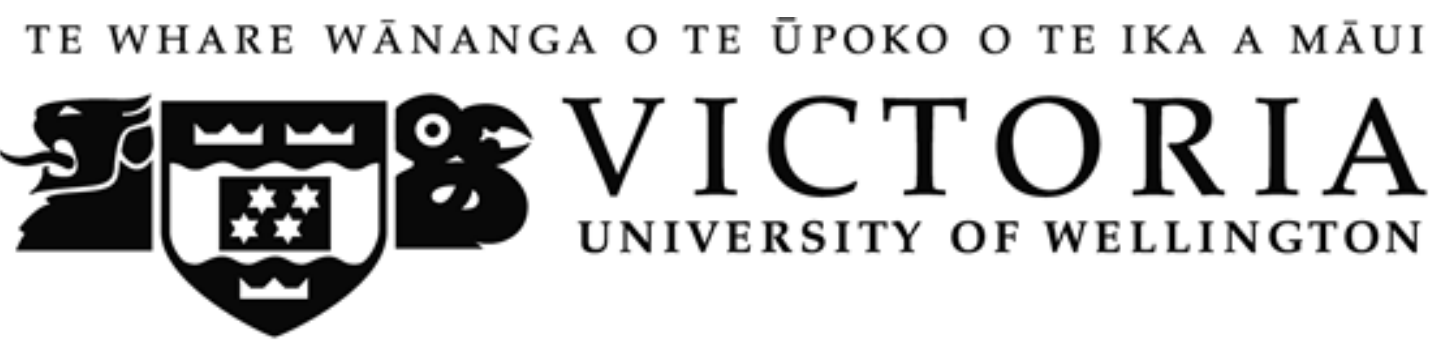

\section{Debriefing statement}

Thank you for participating in this study!

Environmental problems are a result of maladaptive human behaviour. One way to tackle these problems is by fostering values that underlie environmental attitudes and behaviours. This project aims to advance past research by systematically assessing the role of values in motivating individuals' attitudes and behaviours toward the environment.

Past research has shown that there is a strong correspondence between values, attitudes and behavioural intentions. In general, research has shown that selftranscendence values (e.g., social justice, world of peace, loyal, equality, helpful, forgiving, honest, and broad-minded) are positively correlated to environmental attitudes and behaviours. That means people who hold those values are more pro-environmentally friendly. More importantly, research has shown that changes in values can be experimentally manipulated to change behavioural intentions and actual behaviour. I followed this research tradition by using the experimental paradigm developed by Maio et al. (2009) to change behavioural intentions and actual behaviour with the use of values. In their study, Maio et al (2009) tested systematic effects of values, using both manipulation of value change and manipulation of value priming.

$\mathrm{My} \mathrm{PhD}$ research will be one of the first endeavours to assess changes in values using an experimental approach while investigating the effect of this on behavioural intentions and actual behaviour towards the environment. My research will contribute to the existing literature by generating more impactful evidence about the relationship between values and environmental behavioural intentions and actual behaviour. The findings will enable strong research-based knowledge that can be used to improve methods to deploy our values in collective decision making.

The present research can also help campaigns that attempt to elicit behavioural change. The findings from my research may offer a more effective solution to current behaviour change programmes that rely almost exclusively on environmental education - a technique that is typically unsuccessful. Finally, it will also include a critical reflection on the way in which campaigns are designed and their impacts on people's values.

A minor deception was used in this research. You were not told that the value ranking or dessert ranking for Victoria University students is fictitious. This deception was necessary because if you knew the rankings were fictitious it might influence your responses. If completing these questionnaires has caused you to feel any distress you can find help at student counselling services at Victoria University of Wellington (http://www.victoria.ac.nz/st_services/counselling/ ; phone number +64 4463 5310).

Thank you again for participating in this research. The research project is being conducted by Pollyane Diniz, Dr. John McClure and Dr. Taciano L. Milfont from the School of Psychology, Victoria University of Wellington. The results for this study will be available by the end of the year. If you have any questions regarding your involvement in this research, or issues regarding the research in general, please do not hesitate to contact me via e-mail at Pollyane.Diniz@vuw.ac.nz. 


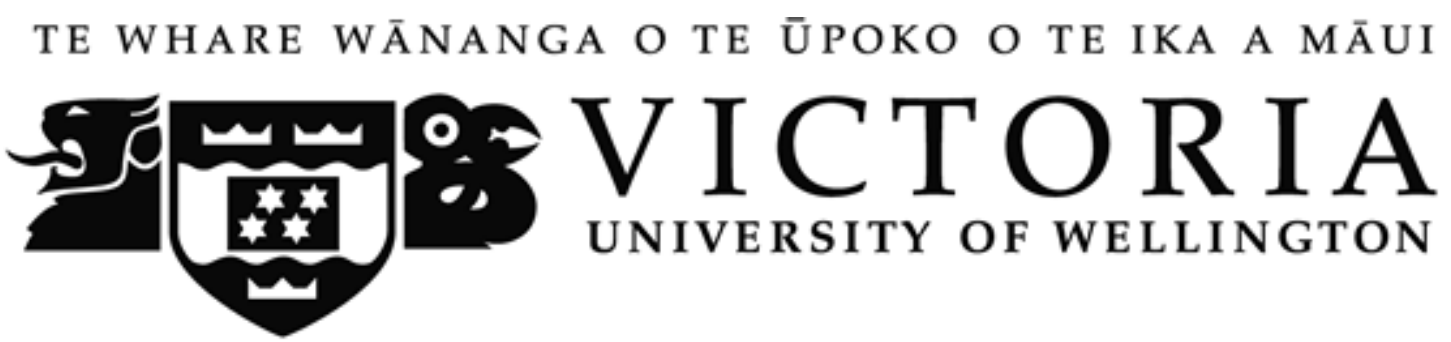

\section{Information Sheet: Data collected for a thesis}

\author{
Pollyane Diniz \\ PhD Student \\ School of Psychology \\ Victoria University of Wellington \\ Pollyane.Diniz@vuw.ac.nz
}

\author{
Dr. Taciano L. Milfont \\ Senior Lecturer \\ School of Psychology \\ Victoria University of Wellington \\ Taciano.Milfont@vuw.ac.nz \\ Phone: 463-6398
}

\author{
Dr. John McClure \\ Professor \\ School of Psychology \\ Victoria University of Wellington \\ john.mcclure@vuw.ac.nz \\ Phone: 4635402
}

\section{What is the purpose of this research?}

This research consists of a questionnaire that asks about opinions, attitudes, and behaviours on a number of social issues that are relevant to our future. The goal is to understand the opinions towards these issues.

Who is conducting the research?

Pollyane Diniz is a PhD student. Dr. Taciano Milfont and Dr. John McClure are supervising this project. This research has been approved by the School of Psychology Human Ethics Committee under delegated authority by Victoria University of Wellington Human Ethics Committee

\section{What is involved if you agree to participate?}

- If you agree to participate in this study you will be asked to complete a short survey. The survey asks you about your identity, specific behaviours, and some demographic questions. The whole study will take you no more than 15 minutes to complete.

- There are no risks in this study that arise from your participation. During the research you are free to withdraw at any point before your survey has been completed.

Privacy and Confidentiality

- This survey is completely anonymous. Please do not put your name anywhere on the survey.

- You will never be identified in my research project or in any other presentation or publication. The information you provide will be coded by number only.

- We will keep your data for at least five years after publication (first publication is expected at the end of 2013), and then it will be destroyed.

- In accordance with the requirements of some scientific journals and organisations, your coded survey may be shared with other competent researchers.

- Your coded data may be used in other, related studies.

- A copy of the coded data will remain in the custody of Pollyane Diniz.

What happens to the information that you provide?

- The data you provide may be used for one or more of the following purposes:

The overall findings may be submitted for publication in a scientific journal, or presented at scientific conferences.

The overall findings may form part of a PhD thesis that will be submitted for assessment.

\section{Consent for participation}

Please note that by completing and returning the questionnaires you agree that the data will be used and analysed.

If you would like to know the results of this study, they will be available at the end of the semester via email upon request.

If you have any further questions regarding this study please contact any one of us above. 


\section{APPENDIX K}

Self-transcendence Values Condition - Experiment 2

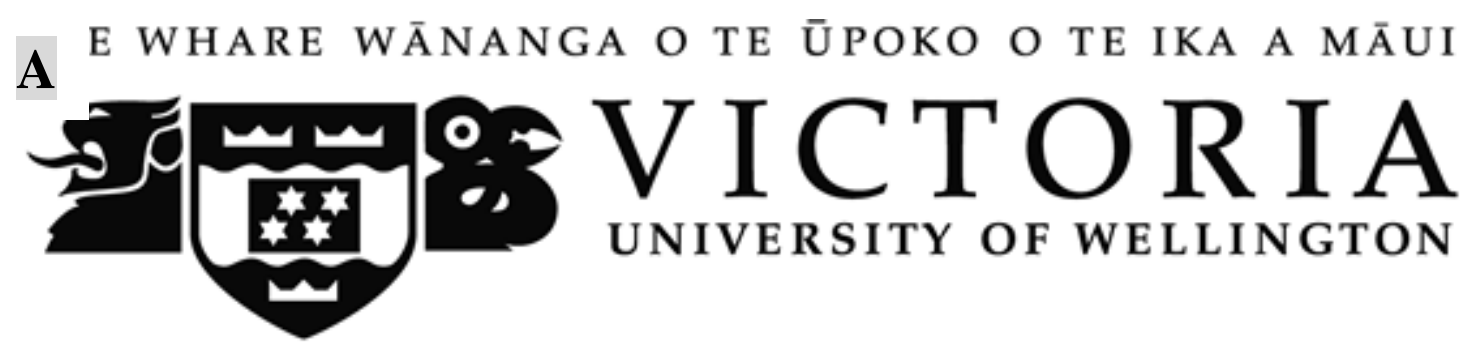

\section{Background questions}

(Please remember that your responses are confidential)

1. How old are you? 2. What is your gender? years.
1. Female

2. Male

3. Are you a member of any environmental organisation (e.g., Greenpeace)?

$$
\text { 1. Yes 2. No }
$$

4. Were you born in New Zealand?

5. How long have you been living in New Zealand?
1. Yes
2. No

6. Which ethnic group do you belong to? Please indicate the group you most strongly identify with.

1. New Zealand European (Pākehā)

2. Pacific Nations

3. Indian
4. Māori

5. Asian

6. Other (please specify):

\section{PART 1. General questions about the environment (Part 1)}

Please indicate the extent to which you would be willing to engage in the following behaviours:

\begin{tabular}{|c|c|c|c|c|}
\hline $\begin{array}{c}\text { Not at all } \\
\text { willing }\end{array}$ & $\begin{array}{c}\text { A little } \\
\text { willing }\end{array}$ & $\begin{array}{c}\text { Moderately } \\
\text { willing }\end{array}$ & Very willing & $\begin{array}{c}\text { Extremely } \\
\text { willing }\end{array}$ \\
\hline
\end{tabular}

Reuse your shopping bags.

$\begin{array}{lllll}0 & 1 & 2 & 3 & 4\end{array}$

Bring empty bottles to a recycling bin.

$\begin{array}{lllll}0 & 1 & 2 & 3 & 4\end{array}$

Buy seasonal produce.

$\begin{array}{lllll}0 & 1 & 2 & 3 & 4\end{array}$ 
Drive in such a way as to keep my fuel consumption as low as possible.

Own energy efficient household devices. $\begin{array}{lllll}0 & 1 & 2 & 3 & 4\end{array}$

Buy domestically grown wooden furniture. $\begin{array}{lllll}0 & 1 & 2 & 3 & 4\end{array}$

Buy meat and produce with eco-labels. $\begin{array}{lllll}0 & 1 & 2 & 3 & 4\end{array}$

Look into the pros and cons of having a private source of solar power.

$\begin{array}{lllll}0 & 1 & 2 & 3 & 4\end{array}$

Talk with friends about problems related to the environment. $\begin{array}{lllll}0 & 1 & 2 & 3 & 4\end{array}$

Own a fuel-efficient automobile (less than 7 I per $100 \mathrm{~km}$; i.e., less than 3 gallons per 100 miles).

Buy milk in returnable bottles.

$\begin{array}{lllll}0 & 1 & 2 & 3 & 4\end{array}$

Point out to someone his or her unecological behaviour. $\begin{array}{lllll}0 & 1 & 2 & 3 & 4\end{array}$

Use renewable energy sources. $\begin{array}{lllll}0 & 1 & 2 & 3 & 4\end{array}$

Be a member of a carpool. $\begin{array}{lllll}0 & 1 & 2 & 3 & 4\end{array}$ $\begin{array}{lllll}0 & 1 & 2 & 3 & 4\end{array}$

\section{PART 2. Personal values (V1)}

We would like to know how important each value is as a guiding principle in your life. Below is a list of 16 values in alphabetical order. We are interested in finding out the relative importance of these values to you.

Study the list carefully. Then place a 1 next to the value which is most important to you, place a 2 next to the second value which is second most important, etc. The value which is least important should be ranked 16 .

When you have completed ranking all the values, go back and check over your list. Feel free to make changes. Please take all the time you need to think about this, so that the end result truly represents your values.

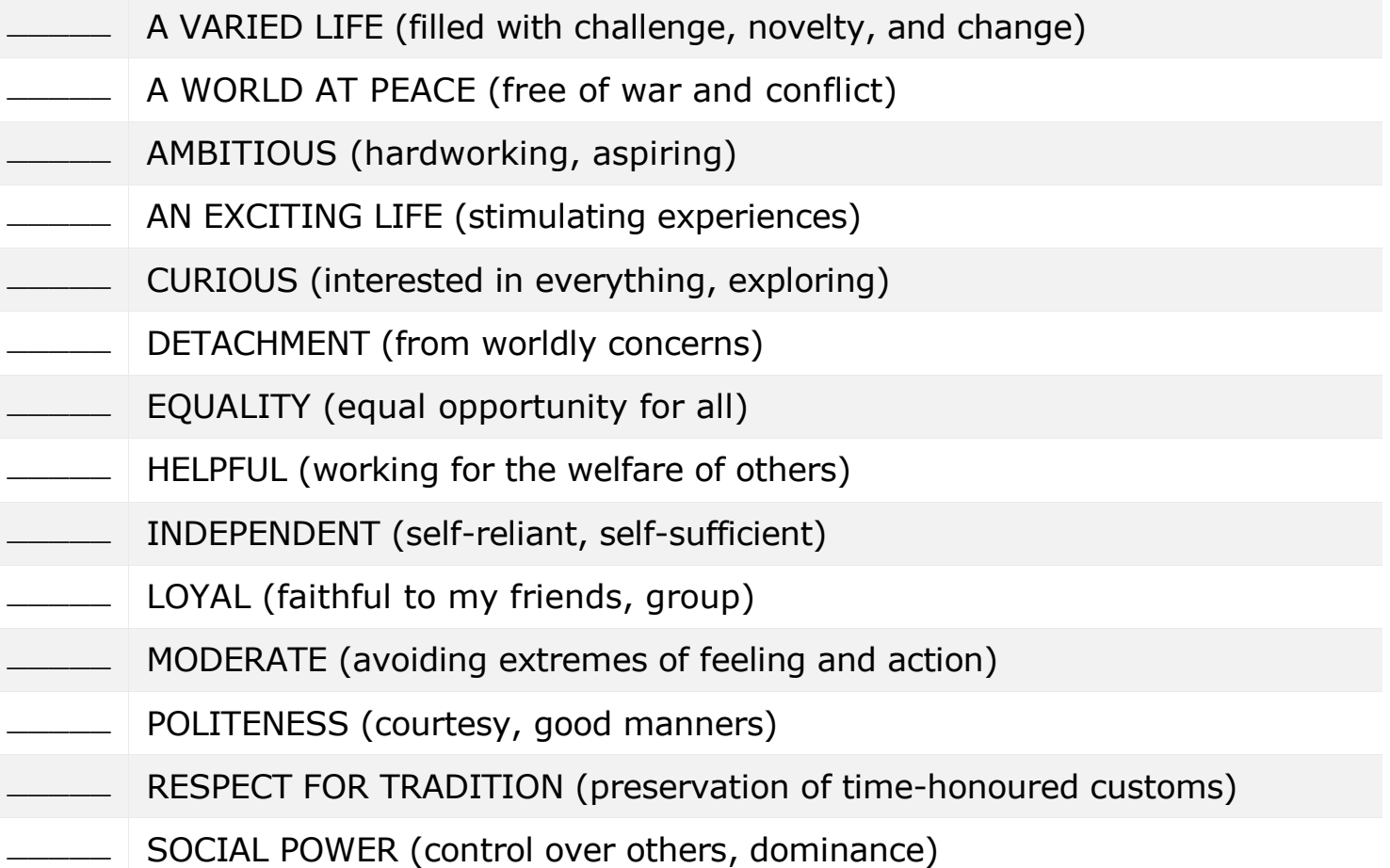


SUCCESSFUL (achieving goals)

Now, I would like to tell you some things we have already found about the value systems of New Zealanders. I am sure that many of you would like to know what they are. The same value system scale that you answered previously was filled out by 298 New Zealanders. The responses of these participants were obtained and averaged together. The table below shows the results.

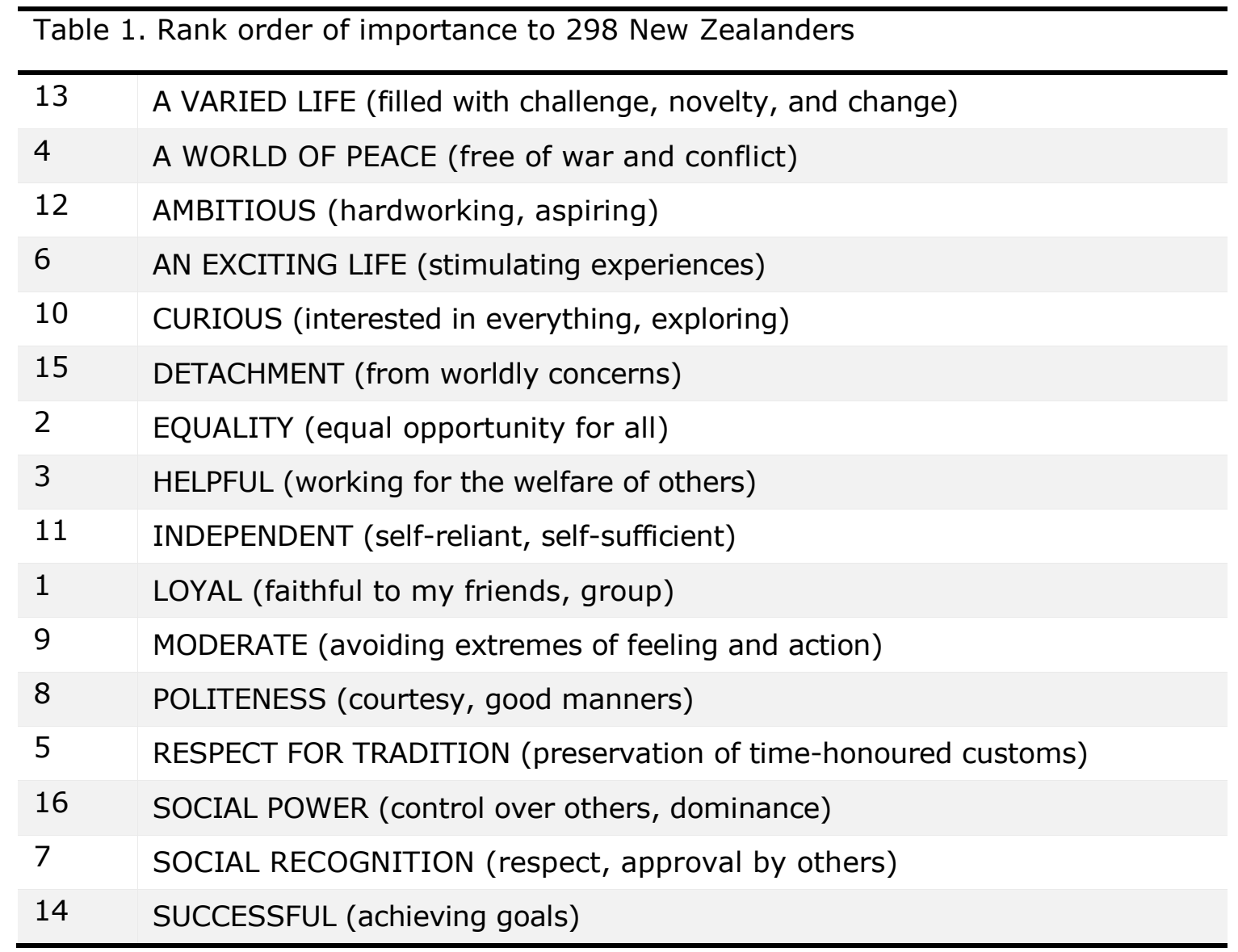

You have your own rankings on the preceding page. Feel free to spend a few minutes comparing your own rankings with those of the 298 New Zealanders, shown in Table 1.

Please identify the four most highly ranked values in the New Zealanders ranking and write the names of the values in a predetermined column below.

The four most highly ranked values for New Zealanders were:

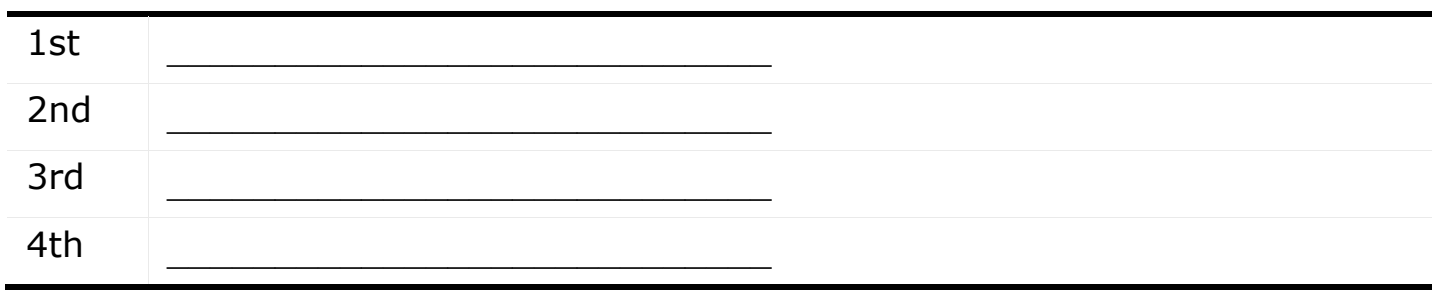


Now, do the same task for your own value rankings. Please identify the four most highly ranked values in your own ranking and write the names of the values in a predetermined column below.

The four most highly ranked values for you were:

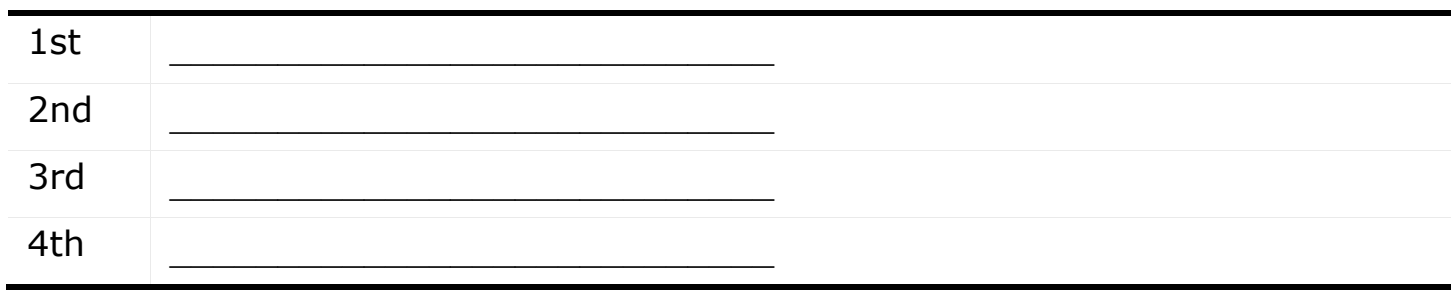

Can you recognize any similarities and differences between your value rankings and New Zealanders' value rankings? Please write down your comments.

Below we have an explanation about the first four preferred values of New Zealanders and about the characteristics of people who rank those values as their most preferred values. Please spend some time reading through the following explanation.

The average of New Zealanders' value ranking shows that the most important values to New Zealanders are Loyalty, Equality, Helpfulness, and A World at Peace. Past research demonstrated that people who believe in these values always emphasise universal human requirements, and are very interested in understanding, appreciating, tolerating, and protecting the welfare of all close others and people in other settings. Therefore, based on the average of New Zealanders' rankings, we can conclude that they have shown their concern for the welfare of all human beings, even those whose way of life differs from theirs.

We are interested to hear from you why you think New Zealanders decided on those four values. Please write your own explanation of why students emphasized the four values. 


\section{PART 3. Personal values (V2)}

Below you will find a new set of 16 values in alphabetical order. These values have not yet been studied in New Zealanders before. We would like to know how important each value is as a guiding principle in your life. We are interested in finding out the relative importance of these values to you.

Study the list carefully. Then place a 1 next to the value which is most important to you, place a 2 next to the second value which is second most important, etc. The value which is least important should be ranked 16 .

When you have completed ranking all the values, go back and check over your list. Feel free to make changes. Please take all the time you need to think about this, so that the end result truly represents your values.

AUTHORITY (the right to lead or command)

BROAD-MINDED (tolerant of different ideas and beliefs)

CAPABLE (competent, effective, efficient)

CHOOSING OWN GOALS (selecting own purposes)

CREATIVITY (uniqueness, imagination)

DARING (seeking adventure, risk)

DEVOUT (holding to religious faith and belief)

FORGIVING (willing to pardon others)

FREEDOM (freedom of action and thought)

HONEST (genuine, sincere)

HONORING OF PARENTS AND ELDERS (showing respect)

INFLUENTIAL (having an impact on people and events)

OBEDIENT (dutiful, meeting obligations)

SOCIAL JUSTICE (correcting injustice, care for the weak)

SOCIAL ORDER (stability of society)

WEALTH (material possessions, money) 


\section{PART 4. Positive and Negative Affect Schedule}

This scale consists of a number of words that describe different feelings and emotions. Read each item and then mark the appropriate answer in the space next to that word. Indicate to what extent you have felt this way during the past few weeks. Use the following scale to record your answers.

\begin{tabular}{|c|c|c|c|c|c|c|}
\hline $\begin{array}{c}1 \\
\text { Very slightly }\end{array}$ & $\begin{array}{c}2 \\
\text { A little }\end{array}$ & & $\begin{array}{l}3 \\
\text { erately }\end{array}$ & $\begin{array}{c}4 \\
\text { Quite a bit }\end{array}$ & & $\begin{array}{c}5 \\
\text { Extremely }\end{array}$ \\
\hline Interested & 1234 & & Irritable & & 1 & 2345 \\
\hline Distressed & 1234 & & Alert & & & 2345 \\
\hline Excited & 1234 & & Ashamec & & & 2345 \\
\hline Upset & 1234 & & Inspired & & & 2345 \\
\hline Strong & 1234 & 5 & Nervous & & & 2345 \\
\hline Guilty & 1234 & 5 & Determir & & 1 & 2345 \\
\hline Scared & 1234 & 5 & Attentive & & 1 & 2345 \\
\hline Hostile & 1234 & 5 & Jittery & & 1 & 2345 \\
\hline Enthusiastic & 1234 & 5 & Active & & 1 & 2345 \\
\hline Proud & 1234 & 5 & Afraid & & & 2345 \\
\hline
\end{tabular}

\section{PART 5. General questions about the environment (Part 2)}

Please indicate the extent to which you would be willing to engage in the following behaviours:

\begin{tabular}{|c|c|c|c|c|c|}
\hline $\begin{array}{c}0 \\
\text { Not at all } \\
\text { willing }\end{array}$ & $\begin{array}{c}1 \\
\text { A little } \\
\text { willing }\end{array}$ & $\begin{array}{c}2 \\
\text { Moderately } \\
\text { willing }\end{array}$ & $\begin{array}{c}3 \\
\text { Very willing }\end{array}$ & & $\begin{array}{c}4 \\
\text { Extremely } \\
\text { willing }\end{array}$ \\
\hline \multicolumn{4}{|c|}{ Collect and recycle used paper. } & 0 & 1234 \\
\hline \multicolumn{4}{|c|}{ Leave the place as clean as it was originally after a picnic. } & 0 & 1234 \\
\hline \multicolumn{4}{|c|}{ Wait until you have a full load before doing your laundry. } & 0 & 1234 \\
\hline \multicolumn{4}{|c|}{ Buy products in refillable packages. } & 0 & 1234 \\
\hline \multicolumn{4}{|c|}{ Ride a bicycle or take public transportation to work or school. } & 0 & 1234 \\
\hline \multicolumn{4}{|c|}{ Boycott companies with an unecological background. } & 0 & 1234 \\
\hline \multicolumn{4}{|c|}{$\begin{array}{l}\text { Use public transportation or ride a bike, when possible in } \\
\text { nearby areas (around } 30 \mathrm{~km} \text {; around } 20 \text { miles). }\end{array}$} & 0 & 1234 \\
\hline
\end{tabular}


Turn down the heat when I leave my apartment for more than $4 \mathrm{~h}$ during winter.

Be a member of an environmental organization.

Drive on freeways at speeds under 100 k.p.h. (62.5 m.p.h.).

$\begin{array}{lllll}0 & 1 & 2 & 3 & 4\end{array}$

Contribute financially to environmental organizations.

$\begin{array}{lllll}0 & 1 & 2 & 3 & 4\end{array}$

Read about environmental issues.

$\begin{array}{lllll}0 & 1 & 2 & 3 & 4\end{array}$

Request an estimate on having solar power installed.

$\begin{array}{lllll}0 & 1 & 2 & 3 & 4\end{array}$

Buy solar panels to produce energy.

$\begin{array}{lllll}0 & 1 & 2 & 3 & 4\end{array}$

\section{PART 6. Group Identification}

Please select the figure below that best describes the extent to which you feel you are a New Zealander.

How much do you identify with being a New Zealander?
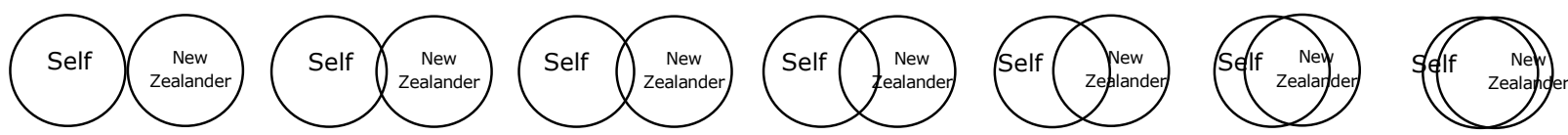

Now, please think about you as a New Zealander and respond to the following statements using the scale below.

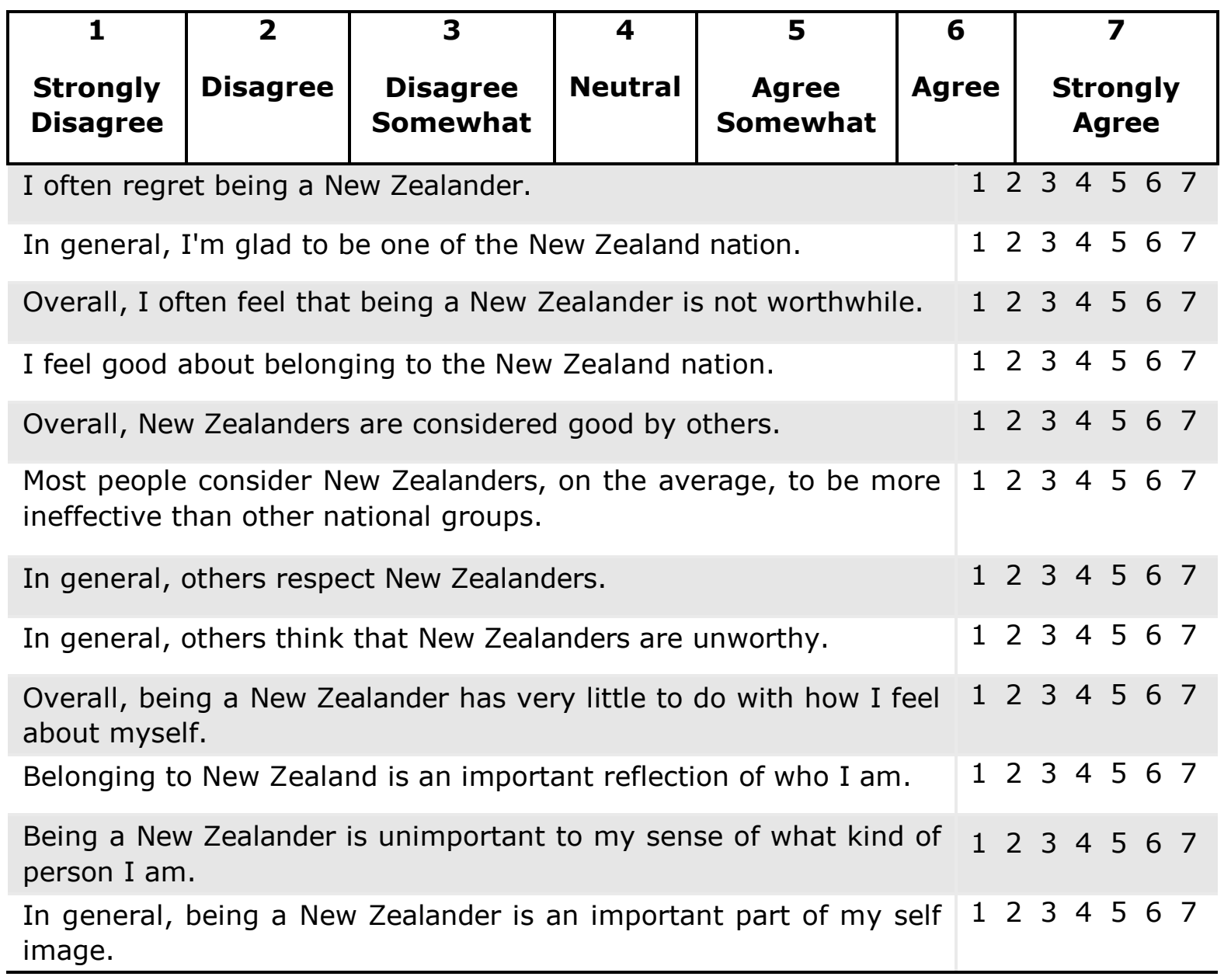




\section{PART 7. General feelings scale}

Below is a list of statements dealing with your general feelings about yourself. If you strongly agree, circle SA. If you agree with the statement, circle A. If you disagree, circle D. If you strongly disagree, circle SD.

\begin{tabular}{|c|c|c|c|}
\hline $\begin{array}{c}\text { SA } \\
\text { Strongly agree }\end{array}$ & $\begin{array}{c}\text { A } \\
\text { Agree }\end{array}$ & $\begin{array}{c}\text { D } \\
\text { Disagree }\end{array}$ & $\begin{array}{c}\text { SD } \\
\text { Strongly disagree }\end{array}$ \\
\hline \multicolumn{3}{|c|}{ On the whole, I am satisfied with myself. } & $S A$ A $D S D$ \\
\hline \multicolumn{3}{|c|}{ At times, I think I am no good at all. } & SA A D SD \\
\hline \multicolumn{3}{|c|}{ I feel that I have a number of good qualities. } & SA A D SD \\
\hline \multicolumn{3}{|c|}{ I am able to do things as well as most other people. } & $S A$ A D SD \\
\hline \multicolumn{3}{|c|}{ I feel I do not have much to be proud of. } & SA A D SD \\
\hline \multicolumn{3}{|c|}{ I certainly feel useless at times. } & SA A D SD \\
\hline \multicolumn{3}{|c|}{$\begin{array}{l}\text { I feel that I'm a person of worth, at least on an equal plane } \\
\text { with others. }\end{array}$} & SA A D SD \\
\hline \multicolumn{3}{|c|}{ I wish I could have more respect for myself. } & SA A D SD \\
\hline \multicolumn{3}{|c|}{ All in all, I am inclined to feel that I am a failure. } & SA A D SD \\
\hline \multicolumn{3}{|c|}{ I take a positive attitude toward myself. } & SA A D SD \\
\hline
\end{tabular}

You will be debriefed about this experiment shortly. However, I'm curious to know your perception of this study. What do you think the purpose of this study was? Did you have any ideas about what kind of results we might be looking at? What did you think we were hoping to learn about? If you are unsure what the purpose is, please state this:

Please write any general comments you would like to make about this questionnaire or the study in general:

\section{Thank you for your time! (:)}




\section{APPENDIX L}

Self-enhancement Values Condition - Experiment 2

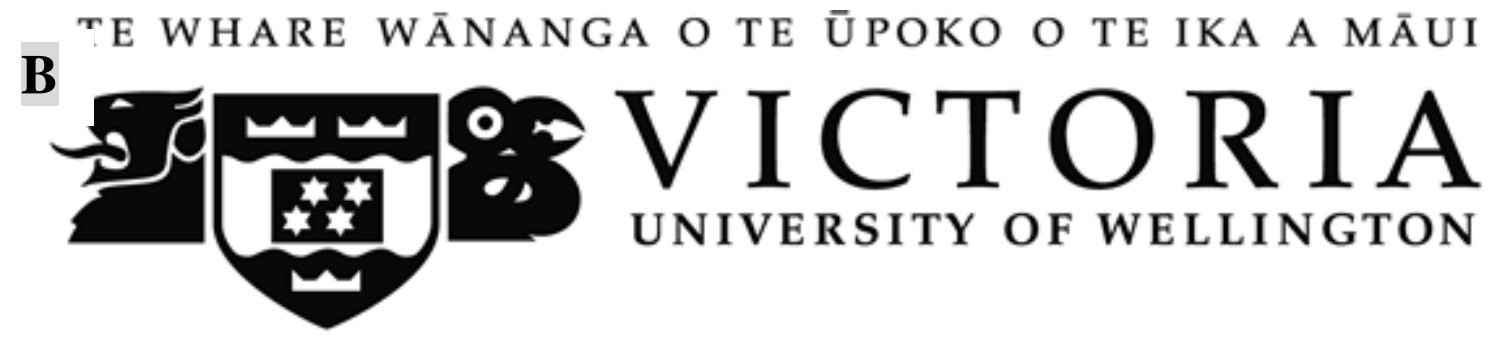

Background questions

(Please remember that your responses are confidential)
1. How old are you?
2. What is your gender?
3. Are you a member of any years.
1. Female
2. Male environmental organisation (e.g., Greenpeace)?
1. Yes
2. No

4. Were you born in New Zealand? Zealand?

5. How long have you been living in New
1. Yes
2. No

6. Which ethnic group do you belong to? Please indicate the group you most strongly identify with.
1. New Zealand European (Pākehā)
4. Māori

2. Pacific Nations

5. Asian

3. Indian

6. Other (please specify):

\section{PART 1. General questions about the environment (Part 1)}

Please indicate the extent to which you would be willing to engage in the following behaviours:

\begin{tabular}{|c|c|c|c|c|}
\hline $\begin{array}{c}0 \\
\text { Not at all } \\
\text { willing }\end{array}$ & $\begin{array}{c}1 \\
\text { A little } \\
\text { willing }\end{array}$ & $\begin{array}{c}2 \\
\text { Moderately } \\
\text { willing }\end{array}$ & $\begin{array}{c}3 \\
\text { Very willing }\end{array}$ & $\begin{array}{c}4 \\
\text { Extremely } \\
\text { willing }\end{array}$ \\
\hline \multicolumn{4}{|c|}{ Reuse your shopping bags. } & $\begin{array}{lllll}0 & 1 & 2 & 3 & 4\end{array}$ \\
\hline \multicolumn{4}{|c|}{ Bring empty bottles to a recycling bin. } & $\begin{array}{lllll}0 & 1 & 2 & 3 & 4\end{array}$ \\
\hline \multicolumn{4}{|c|}{ Buy seasonal produce. } & $\begin{array}{lllll}0 & 1 & 2 & 3 & 4\end{array}$ \\
\hline
\end{tabular}


Drive in such a way as to keep my fuel consumption as low as possible.

Own energy efficient household devices.

$\begin{array}{lllll}0 & 1 & 2 & 3 & 4\end{array}$

Buy domestically grown wooden furniture.

$\begin{array}{lllll}0 & 1 & 2 & 3 & 4\end{array}$

Buy meat and produce with eco-labels.

$\begin{array}{lllll}0 & 1 & 2 & 3 & 4\end{array}$

Look into the pros and cons of having a private source of solar power.

$\begin{array}{lllll}0 & 1 & 2 & 3 & 4\end{array}$

Talk with friends about problems related to the environment.

$\begin{array}{lllll}0 & 1 & 2 & 3 & 4\end{array}$

Own a fuel-efficient automobile (less than 7 I per 100 km; i.e., less than 3 gallons per 100 miles).

Buy milk in returnable bottles.

$\begin{array}{lllll}0 & 1 & 2 & 3 & 4\end{array}$

Point out to someone his or her unecological behaviour.

$\begin{array}{lllll}0 & 1 & 2 & 3 & 4\end{array}$

Use renewable energy sources.

$\begin{array}{lllll}0 & 1 & 2 & 3 & 4\end{array}$

Be a member of a carpool.

$\begin{array}{lllll}0 & 1 & 2 & 3 & 4\end{array}$

$\begin{array}{lllll}0 & 1 & 2 & 3 & 4\end{array}$

\section{PART 2. Personal values (V1)}

We would like to know how important each value is as a guiding principle in your life. Below is a list of 16 values in alphabetical order. We are interested in finding out the relative importance of these values to you.

Study the list carefully. Then place a 1 next to the value which is most important to you, place a 2 next to the second value which is second most important, etc. The value which is least important should be ranked 16 .

When you have completed ranking all the values, go back and check over your list. Feel free to make changes. Please take all the time you need to think about this, so that the end result truly represents your values.

A VARIED LIFE (filled with challenge, novelty, and change)

A WORLD AT PEACE (free of war and conflict)

AMBITIOUS (hardworking, aspiring)

AN EXCITING LIFE (stimulating experiences)

CURIOUS (interested in everything, exploring)

DETACHMENT (from worldly concerns)

EQUALITY (equal opportunity for all)

HELPFUL (working for the welfare of others)

INDEPENDENT (self-reliant, self-sufficient)

LOYAL (faithful to my friends, group)

MODERATE (avoiding extremes of feeling and action)

POLITENESS (courtesy, good manners)

RESPECT FOR TRADITION (preservation of time-honoured customs)

SOCIAL POWER (control over others, dominance)

SOCIAL RECOGNITION (respect, approval by others) 


\section{SUCCESSFUL (achieving goals)}

Now, I would like to tell you some things we have already found about the value systems of New Zealanders. I am sure that many of you would like to know what they are. The same value system scale that you answered previously was filled out by 298 New Zealanders. The responses of these participants were obtained and averaged together. The table below shows the results.

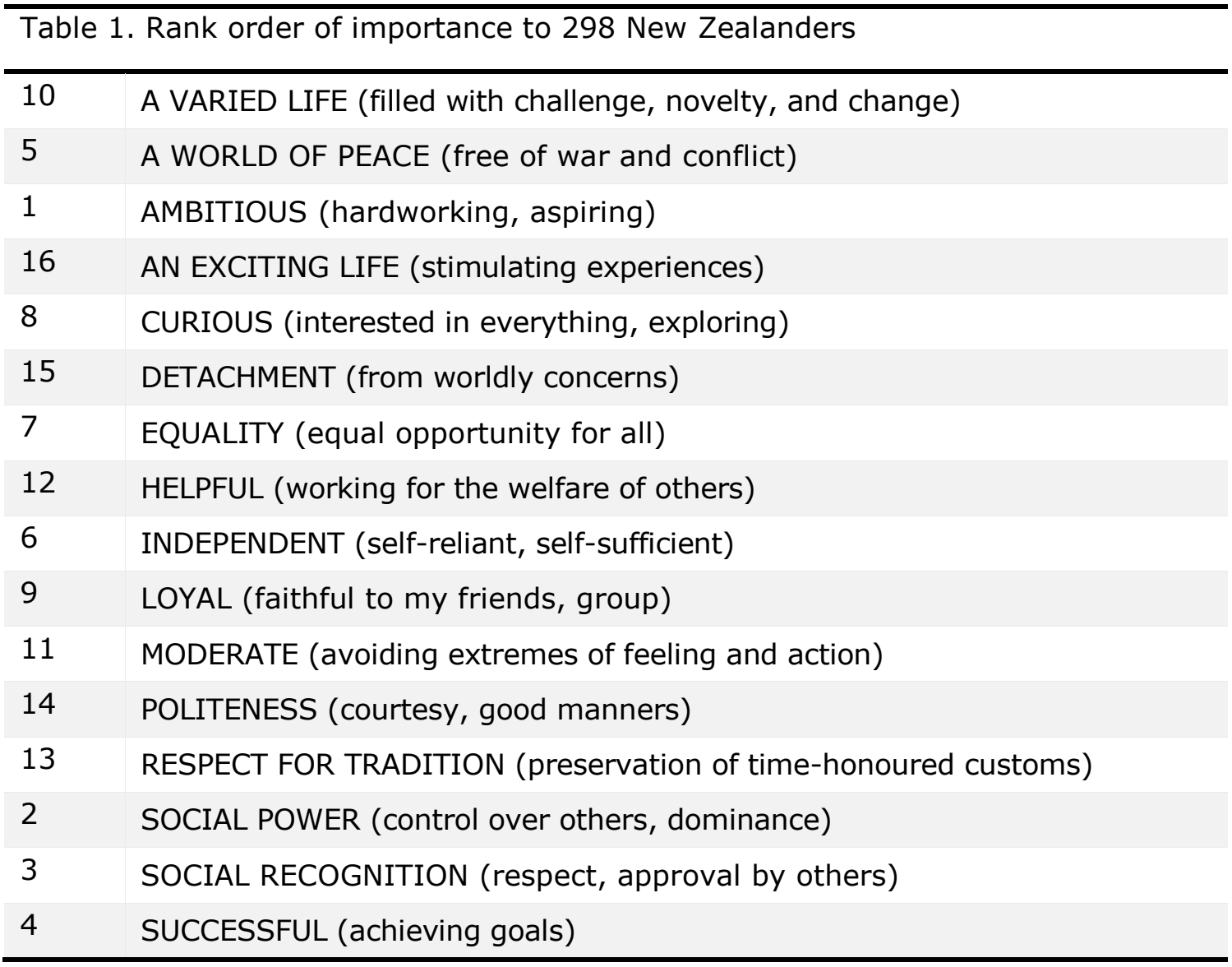

You have your own rankings on the preceding page. Feel free to spend a few minutes comparing your own rankings with those of the 298 New Zealanders, shown in Table 1.

Please identify the four most highly ranked values in the New Zealanders ranking and write the names of the values in a predetermined column below.

The four most highly ranked values for New Zealanders were:

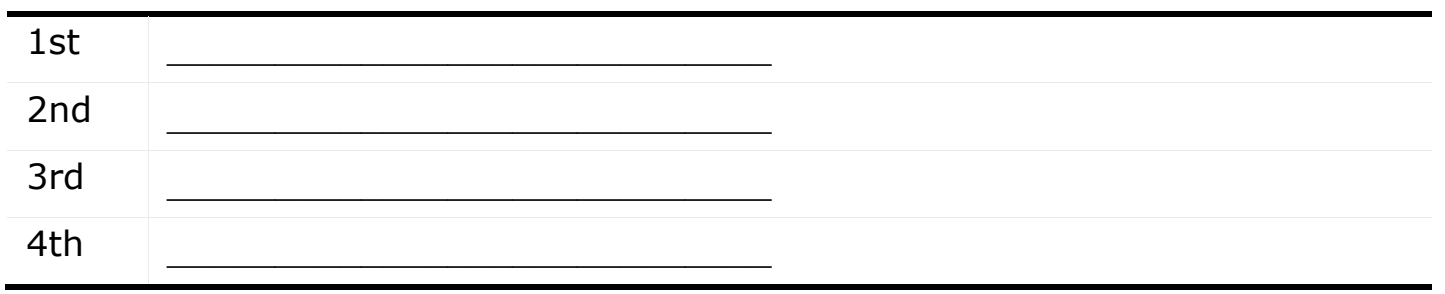


Now, do the same task for your own value rankings. Please identify the four most highly ranked values in your own ranking and write the names of the values in a predetermined column below.

The four most highly ranked values for you were:

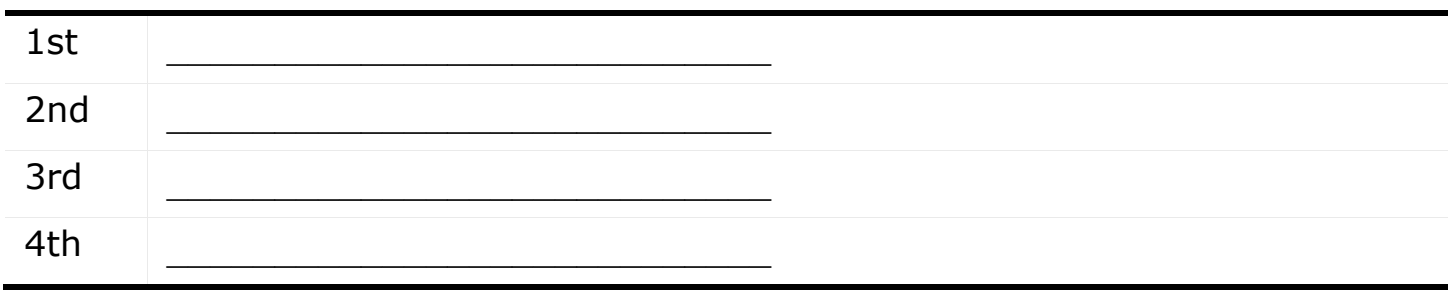

Can you recognize any similarities and differences between your value rankings and New Zealanders' value rankings? Please write down your comments.

Below we have an explanation about the first four preferred values of New Zealanders and about the characteristics of people who rank those values as their most preferred values. Please spend some time reading through the following explanation.

The average of the New Zealanders' value ranking shows that the most important values to New Zealanders are Ambition, Social power, Social recognition, and Successfulness. Past research demonstrated that people who believe in these values always emphasise achievement and personal success through demonstrating competence according to social standards and attainment of social status and prestige. Therefore, based on the average of New Zealanders' rankings, we can conclude that they have shown their concern for active demonstration of competence in concrete interaction and attainment of a dominant position within a social system.

We are interested to hear from you why you think New Zealanders decided on those four values. Please write your own explanation of why students emphasized the four values. 


\section{PART 3. Personal values (V2)}

Below you will find a new set of 16 values in alphabetical order. These values have not yet been studied in New Zealanders before. We would like to know how important each value is as a guiding principle in your life. We are interested in finding out the relative importance of these values to you.

Study the list carefully. Then place a 1 next to the value which is most important to you, place a 2 next to the second value which is second most important, etc. The value which is least important should be ranked 16 .

When you have completed ranking all the values, go back and check over your list. Feel free to make changes. Please take all the time you need to think about this, so that the end result truly represents your values.

AUTHORITY (the right to lead or command)

BROAD-MINDED (tolerant of different ideas and beliefs)

CAPABLE (competent, effective, efficient)

CHOOSING OWN GOALS (selecting own purposes)

CREATIVITY (uniqueness, imagination)

DARING (seeking adventure, risk)

DEVOUT (holding to religious faith and belief)

FORGIVING (willing to pardon others)

FREEDOM (freedom of action and thought)

HONEST (genuine, sincere)

HONORING OF PARENTS AND ELDERS (showing respect)

INFLUENTIAL (having an impact on people and events)

OBEDIENT (dutiful, meeting obligations)

SOCIAL JUSTICE (correcting injustice, care for the weak)

SOCIAL ORDER (stability of society)

WEALTH (material possessions, money) 


\section{PART 4. Positive and Negative Affect Schedule}

This scale consists of a number of words that describe different feelings and emotions. Read each item and then mark the appropriate answer in the space next to that word. Indicate to what extent you have felt this way during the past few weeks. Use the following scale to record your answers.

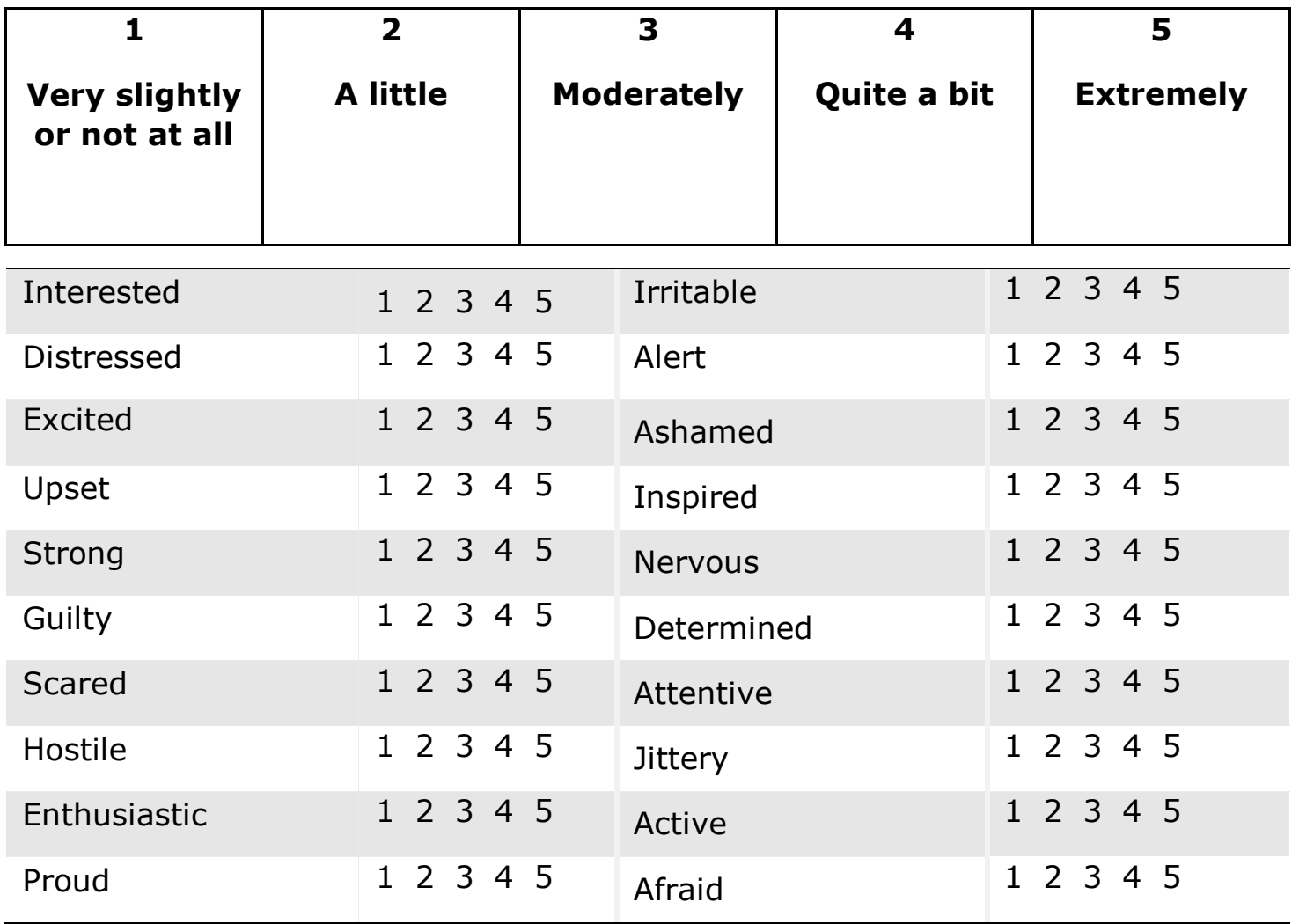

\section{PART 5. General questions about the environment (Part 2)}

Please indicate the extent to which you would be willing to engage in the following behaviours:

\begin{tabular}{|c|c|c|c|c|}
\hline $\begin{array}{c}0 \\
\text { Not at all } \\
\text { willing }\end{array}$ & $\begin{array}{c}1 \\
\text { A little } \\
\text { willing }\end{array}$ & $\begin{array}{c}2 \\
\text { Moderately } \\
\text { willing }\end{array}$ & $\begin{array}{c}3 \\
\text { Very willing }\end{array}$ & $\begin{array}{c}4 \\
\text { Extremely } \\
\text { willing }\end{array}$ \\
\hline \multicolumn{4}{|c|}{ Collect and recycle used paper. } & 01234 \\
\hline \multicolumn{4}{|c|}{ Leave the place as clean as it was originally after a picnic. } & 01234 \\
\hline \multicolumn{4}{|c|}{ Wait until you have a full load before doing your laundry. } & 011234 \\
\hline \multicolumn{4}{|c|}{ Buy products in refillable packages. } & 012234 \\
\hline \multicolumn{4}{|c|}{ Ride a bicycle or take public transportation to work or school. } & 01234 \\
\hline \multicolumn{4}{|c|}{ Boycott companies with an unecological background. } & 012234 \\
\hline \multicolumn{4}{|c|}{$\begin{array}{l}\text { Use public transportation or ride a bike, when possible in } \\
\text { nearby areas (around } 30 \mathrm{~km} \text {; around } 20 \text { miles). }\end{array}$} & 01234 \\
\hline
\end{tabular}


Turn down the heat when I leave my apartment for more than $4 \mathrm{~h}$ during winter.

Be a member of an environmental organization. $\begin{array}{lllll}0 & 1 & 2 & 3 & 4\end{array}$

Drive on freeways at speeds under 100 k.p.h. (62.5 m.p.h.).

$\begin{array}{lllll}0 & 1 & 2 & 3 & 4\end{array}$

Contribute financially to environmental organizations.

$\begin{array}{lllll}0 & 1 & 2 & 3 & 4\end{array}$

Read about environmental issues.

$\begin{array}{lllll}0 & 1 & 2 & 3 & 4\end{array}$

Request an estimate on having solar power installed.

$\begin{array}{lllll}0 & 1 & 2 & 3 & 4\end{array}$

Buy solar panels to produce energy.

$\begin{array}{lllll}0 & 1 & 2 & 3 & 4\end{array}$

\section{PART 6. Group Identification}

Please select the figure below that best describes the extent to which you feel you are a New Zealander.

How much do you identify with being a New Zealander?
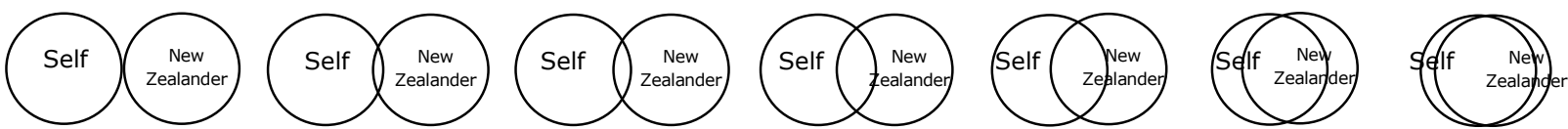

Now, please think about you as a New Zealander and respond to the following statements using the scale below.

\begin{tabular}{|c|c|c|c|c|c|c|}
\hline $\mathbf{1}$ & $\mathbf{2}$ & $\mathbf{3}$ & $\mathbf{4}$ & $\mathbf{5}$ & $\mathbf{6}$ & $\mathbf{7}$ \\
$\begin{array}{c}\text { Strongly } \\
\text { Disagree }\end{array}$ & Disagree & $\begin{array}{c}\text { Disagree } \\
\text { Somewhat }\end{array}$ & Neutral & $\begin{array}{c}\text { Agree } \\
\text { Somewhat }\end{array}$ & $\begin{array}{c}\text { Agree } \\
\text { Strongly } \\
\text { Agree }\end{array}$ \\
\hline
\end{tabular}

I often regret being a New Zealander.

$\begin{array}{lllllll}1 & 2 & 3 & 4 & 5 & 6 & 7\end{array}$

In general, I'm glad to be one of the New Zealand nation.

123345567

Overall, I often feel that being a New Zealander is not worthwhile.

12334567

I feel good about belonging to the New Zealand nation.

123345567

Overall, New Zealanders are considered good by others.

12334567

Most people consider New Zealanders, on the average, to be more $1 \begin{array}{lllllll}2 & 34 & 5 & 6\end{array}$ ineffective than other national groups.

In general, others respect New Zealanders.

12334567

In general, others think that New Zealanders are unworthy.

123345567

Overall, being a New Zealander has very little to do with how I feel $\begin{array}{lllllll}2 & 2 & 4 & 5 & 6 & 7\end{array}$ about myself.

Belonging to New Zealand is an important reflection of who I am. $\quad \begin{array}{lllllll}2 & 3 & 4 & 5 & 6\end{array}$

Being a New Zealander is unimportant to my sense of what kind of $\begin{array}{lllllll}1 & 2 & 34 & 5 & 6\end{array}$ person I am.

In general, being a New Zealander is an important part of my self $1 \begin{array}{lllllll}1 & 2 & 3 & 4 & 5 & 6 & 7\end{array}$ image. 


\section{PART 7. General feelings scale}

Below is a list of statements dealing with your general feelings about yourself. If you strongly agree, circle SA. If you agree with the statement, circle A. If you disagree, circle D. If you strongly disagree, circle SD.

\begin{tabular}{l|c|c|}
\hline \multicolumn{1}{c|}{$\begin{array}{c}\text { SA } \\
\text { Strongly agree }\end{array}$} & \multicolumn{1}{c|}{$\begin{array}{c}\text { D } \\
\text { Disagree }\end{array}$} & $\begin{array}{c}\text { SD } \\
\text { Strongly disagree }\end{array}$ \\
\hline On the whole, I am satisfied with myself. & SA A D SD \\
\hline At times, I think I am no good at all. & SA A D SD \\
I feel that I have a number of good qualities. & SA A D SD \\
I am able to do things as well as most other people. & SA A D SD \\
I feel I do not have much to be proud of. & SA A D SD \\
I certainly feel useless at times. & SA A D SD \\
I feel that I'm a person of worth, at least on an equal plane & SA A D SD \\
with others. & SA A D SD \\
I wish I could have more respect for myself. & SA A D SD \\
All in all, I am inclined to feel that I am a failure. & SA A D SD \\
\hline I take a positive attitude toward myself.
\end{tabular}

You will be debriefed about this experiment shortly. However, I'm curious to know your perception of this study. What do you think the purpose of this study was? Did you have any ideas about what kind of results we might be looking at? What did you think we were hoping to learn about? If you are unsure what the purpose is, please state this:

Please write any general comments you would like to make about this questionnaire or the study in general: 


\title{
APPENDIX M
}

\author{
Control Condition - Experiment 2
}

C

TE WHARE WĀNANGA O TE ŪPOKO O TE IKA A MĀUI $\left.\underbrace{*}_{*}\right|_{*} ^{*}$

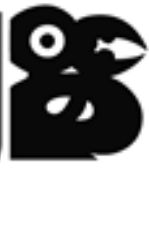

\section{Background questions}

(Please remember that your responses are confidential)

1. How old are you?

2. What is your gender? years.

2. Male

3. Are you a member of any environmental organisation (e.g., Greenpeace)?

$$
\text { 1. Yes 2. No }
$$

4. Were you born in New Zealand? Zealand?

5. How long have you been living in New
1. Yes
2. No

6. Which ethnic group do you belong to? Please indicate the group you most strongly identify with.
1. New Zealand European (Pākehā)
2. Pacific Nations
4. Māori
5. Asian

3. Indian

6. Other (please specify):

\section{PART 1. General questions about the environment (Part 1)}

Please indicate the extent to which you would be willing to engage in the following

\begin{tabular}{|c|c|c|c|c|}
\hline $\begin{array}{c}0 \\
\text { Not at all } \\
\text { willing }\end{array}$ & $\begin{array}{c}1 \\
\text { A little } \\
\text { willing }\end{array}$ & $\begin{array}{c}\mathbf{2} \\
\text { Moderately } \\
\text { willing }\end{array}$ & $\begin{array}{c}3 \\
\text { Very willing }\end{array}$ & $\begin{array}{c}4 \\
\text { Extremely } \\
\text { willing }\end{array}$ \\
\hline \multicolumn{4}{|c|}{ Reuse your shopping bags. } & 012234 \\
\hline \multicolumn{4}{|c|}{ Bring empty bottles to a recycling bin. } & 01234 \\
\hline \multicolumn{4}{|c|}{ Buy seasonal produce. } & 01234 \\
\hline \multicolumn{4}{|c|}{$\begin{array}{l}\text { Drive in such a way as to keep my fuel consumption as low } \\
\text { as possible. }\end{array}$} & 012234 \\
\hline \multicolumn{4}{|c|}{ Own energy efficient household devices. } & 012234 \\
\hline
\end{tabular}
behaviours: 
Buy domestically grown wooden furniture.

$\begin{array}{lllll}0 & 1 & 2 & 3 & 4\end{array}$

Buy meat and produce with eco-labels.

$\begin{array}{lllll}0 & 1 & 2 & 3 & 4\end{array}$

Look into the pros and cons of having a private source of solar power.

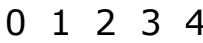

Talk with friends about problems related to the environment.

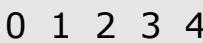

Own a fuel-efficient automobile (less than 7 I per $100 \mathrm{~km}$; i.e., less than 3 gallons per 100 miles).

Buy milk in returnable bottles.

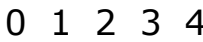

Point out to someone his or her unecological behaviour.

012234

Use renewable energy sources.

$\begin{array}{lllll}0 & 1 & 2 & 3 & 4\end{array}$

Be a member of a carpool.

\section{PART 2. Personal values (V1)}

We would like to know how important each value is as a guiding principle in your life. Below is a list of 16 values in alphabetical order. We are interested in finding out the relative importance of these values to you.

Study the list carefully. Then place a 1 next to the value which is most important to you, place a 2 next to the second value which is second most important, etc. The value which is least important should be ranked 16 .

When you have completed ranking all the values, go back and check over your list. Feel free to make changes. Please take all the time you need to think about this, so that the end result truly represents your values.

A VARIED LIFE (filled with challenge, novelty, and change)

A WORLD AT PEACE (free of war and conflict)

AMBITIOUS (hardworking, aspiring)

AN EXCITING LIFE (stimulating experiences)

CURIOUS (interested in everything, exploring)

DETACHMENT (from worldly concerns)

EQUALITY (equal opportunity for all)

HELPFUL (working for the welfare of others)

INDEPENDENT (self-reliant, self-sufficient)

LOYAL (faithful to my friends, group)

MODERATE (avoiding extremes of feeling and action)

POLITENESS (courtesy, good manners)

RESPECT FOR TRADITION (preservation of time-honoured customs)

SOCIAL POWER (control over others, dominance)

SOCIAL RECOGNITION (respect, approval by others)

SUCCESSFUL (achieving goals) 


\section{PART 3. Dessert preferences (Part 1)}

Below is a list of 16 desserts in alphabetical order. We are interested in finding out the relative preferences of these desserts to you.

Study the list carefully. Then place a 1 next to the dessert which you prefer most, place a 2 next to the second dessert which is second most preferred, etc. The dessert which is least preferred should be ranked 16 .

When you completed ranking all the options, go back and check over your list. Feel free to make changes. Please take all the time you need to think about this, so that the end result truly represents your preferences.

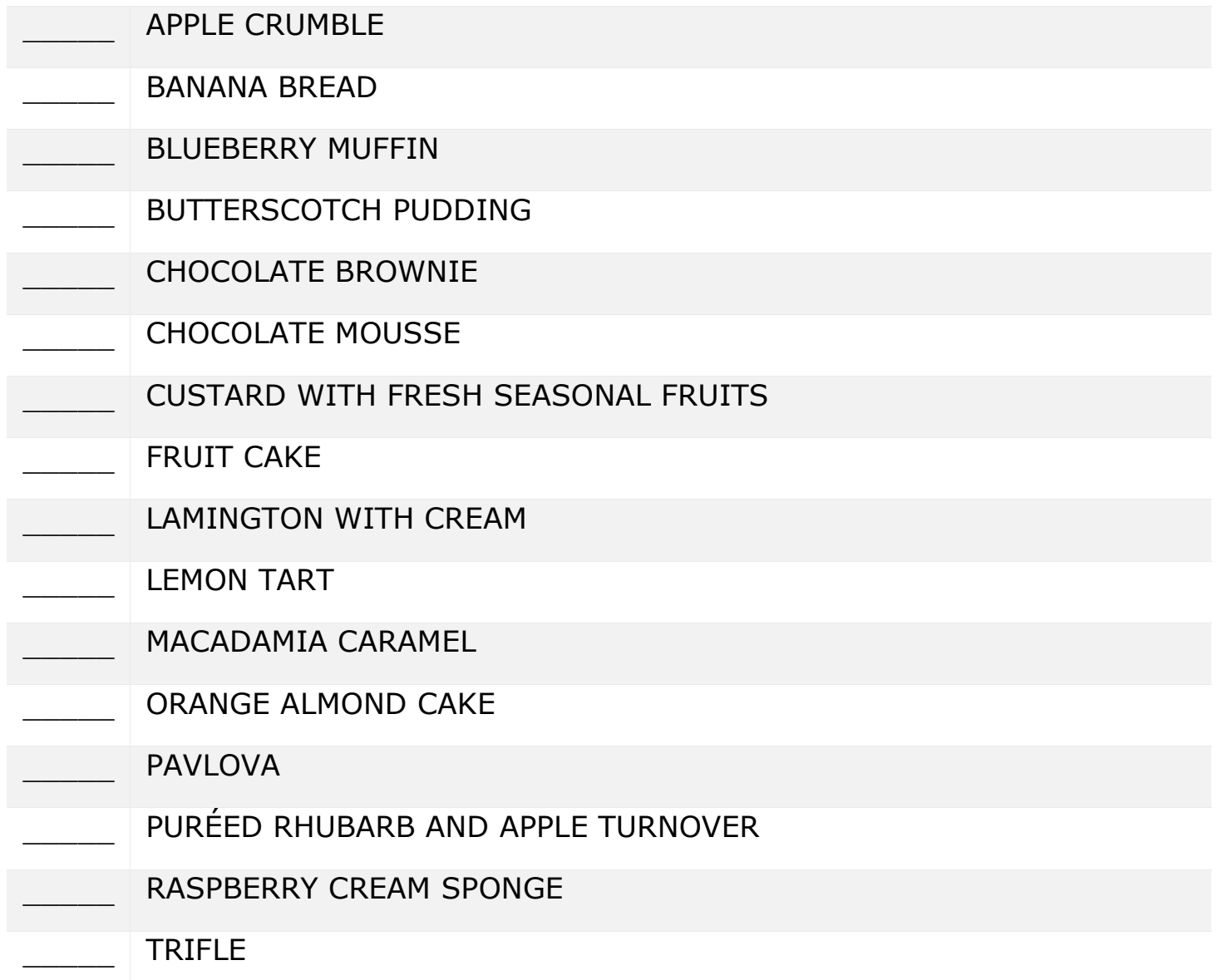

Now, I would like to tell you some things we have already found about the desserts preferences of New Zealanders. I am sure that many of you would like to know what they are.

The same dessert preferences scale that you answered previously was filled out by 298 New Zealanders. The responses of these participants were obtained and averaged together. The table below shows the results. 


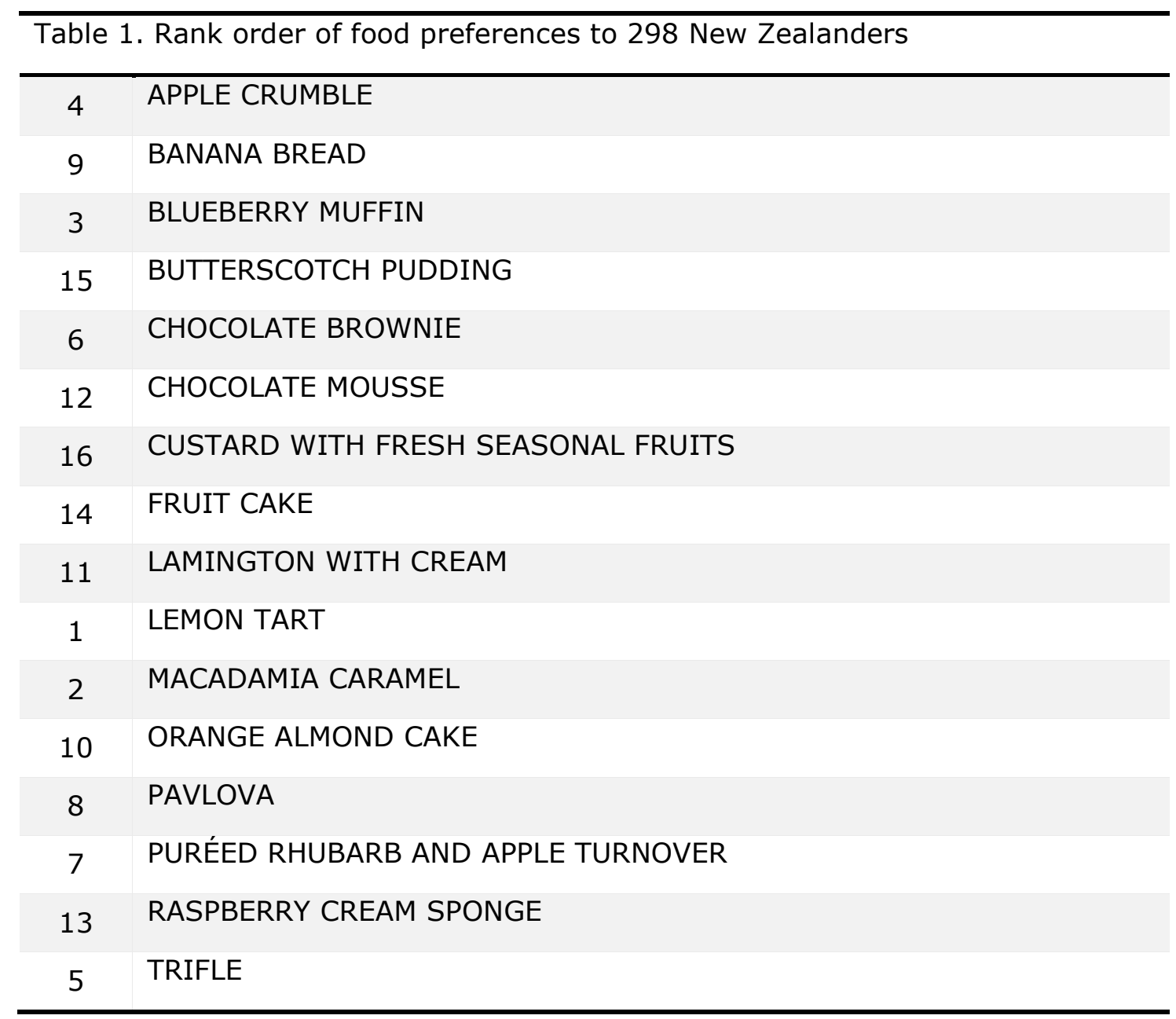

You have your own rankings on the preceding page. Feel free to spend a few minutes comparing your own rankings with those of the 298 New Zealanders, shown in Table 1.

Please identify the four most highly ranked dessert preferences in the New Zealanders ranking and write the names of the dishes in a predetermined column below.

The four most highly ranked desserts for New Zealanders were:

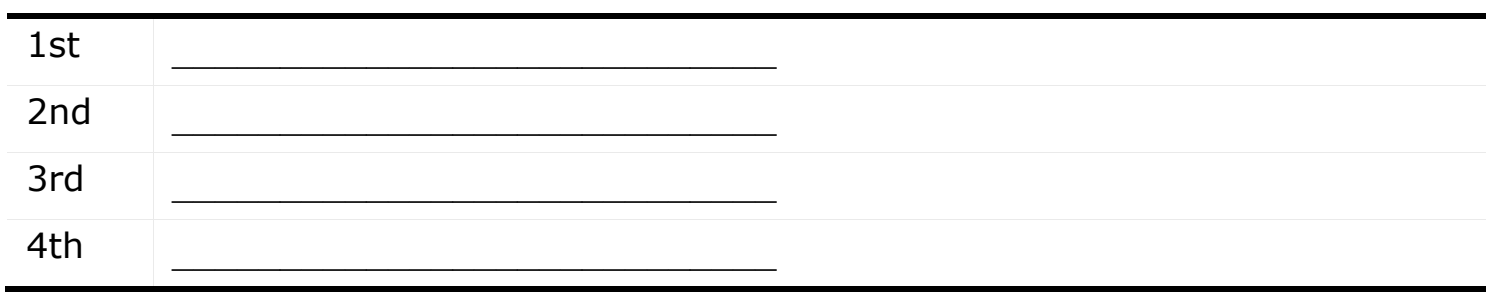

Now, do the same task for your own dessert preferences rankings. Please identify the four most highly ranked dessert preferences in your own ranking and write the names of the desserts in a predetermined column below. 
The four most highly ranked desserts for you were:

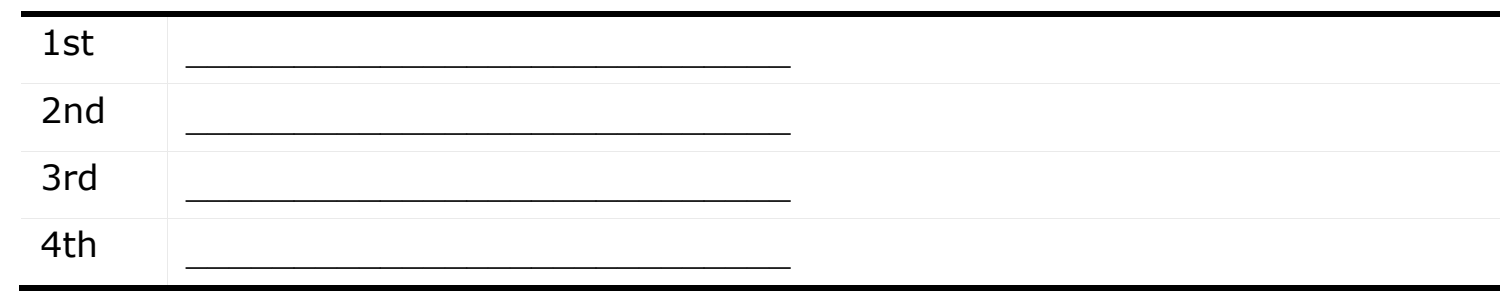

Can you recognize any similarities and differences between your dessert preferences rankings and the New Zealanders rankings? Please write down your comments.

Below we have an explanation about the first four preferred options of desserts for New Zealanders and about the characteristics of people who rank those desserts as their most preferred options. Please spend some time reading through the following explanation.

The average of the New Zealanders' evaluations of what would be the best options for dessert showed lemon tart, macadamia caramel, blueberry muffin and apple crumble as the four favourites. Past research demonstrated that people who decide on flavours of food base their choices on personal preferences and dietary requirements. Therefore, based on the average of New Zealanders' rankings, we can conclude that they have shown their preferences while taking into account their favourite desserts in their country.

We are interested to hear from you why you think New Zealanders chose those four options for desserts. Please write your own explanation of why New Zealanders emphasized the four preferences. 


\section{PART 4. Dessert preferences (Part 2)}

Below you will find a new set of 16 desserts in alphabetical order. These desserts have not yet been studied in New Zealanders before. We are interested in finding out the relative preferences of these desserts to you.

Study the list carefully. Then place a 1 next to the dessert which you prefer most, place a 2 next to the second dessert which is second most preferred, etc. The dessert which is least preferred should be ranked 16 .

When you have completed ranking all the options, go back and check over your list. Feel free to make changes. Please take all the time you need to think about this, so that the end result truly represents your preferences.

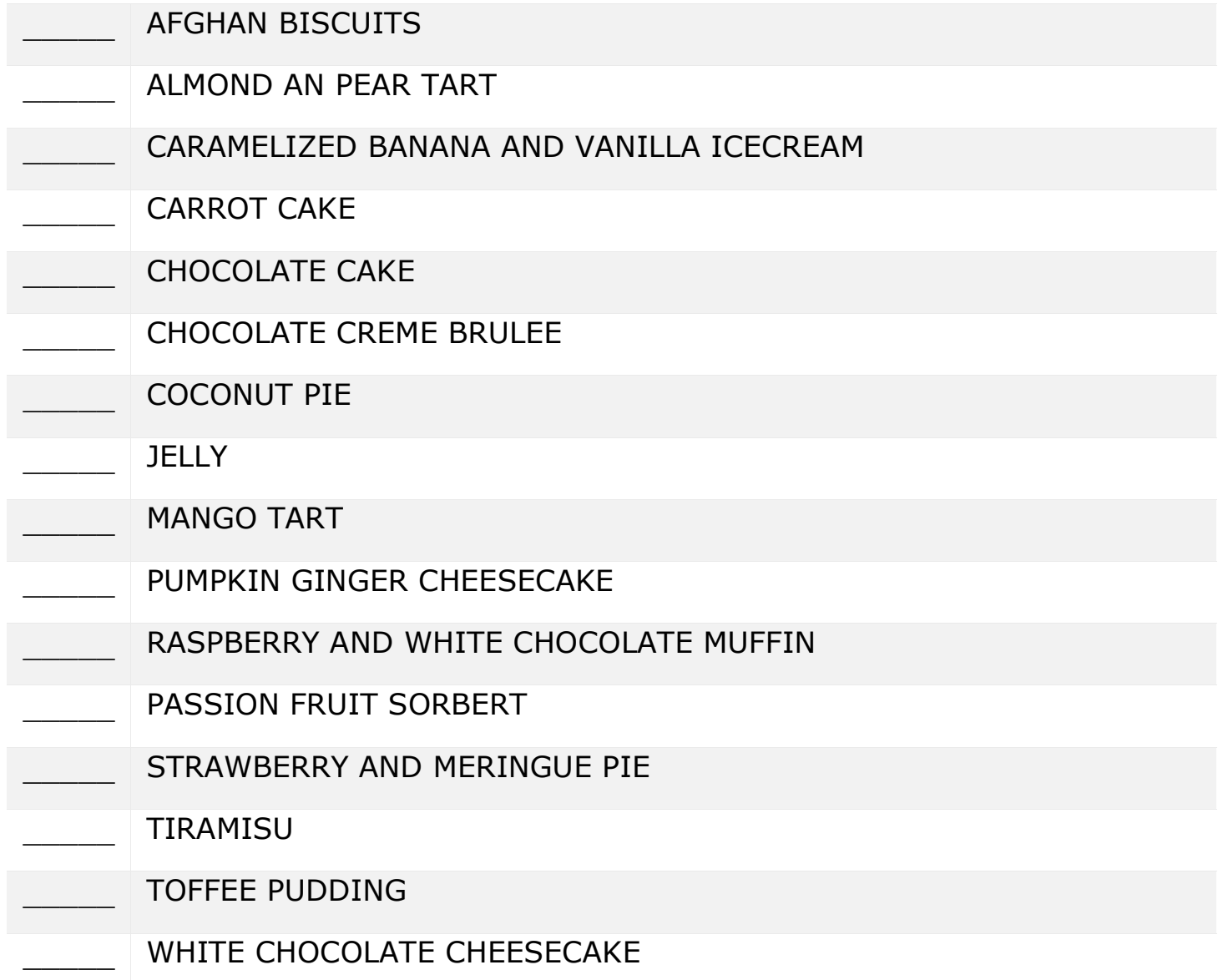

\section{PART 5. Personal values (V2)}

Below you will find a new set of 16 values in alphabetical order. These values have not yet been studied in New Zealanders before. We would like to know how important each value is as a guiding principle in your life. We are interested in finding out the relative importance of these values to you.

Study the list carefully. Then place a 1 next to the value which is most important to you, place a 2 next to the second value which is second most important, etc. The value which is least important should be ranked 16 . 
When you have completed ranking all the values, go back and check over your list. Feel free to make changes. Please take all the time you need to think about this, so that the end result truly represents your values.

AUTHORITY (the right to lead or command)

BROAD-MINDED (tolerant of different ideas and beliefs)

CAPABLE (competent, effective, efficient)

CHOOSING OWN GOALS (selecting own purposes)

CREATIVITY (uniqueness, imagination)

DARING (seeking adventure, risk)

DEVOUT (holding to religious faith and belief)

FORGIVING (willing to pardon others)

FREEDOM (freedom of action and thought)

HONEST (genuine, sincere)

HONORING OF PARENTS AND ELDERS (showing respect)

INFLUENTIAL (having an impact on people and events)

OBEDIENT (dutiful, meeting obligations)

SOCIAL JUSTICE (correcting injustice, care for the weak)

SOCIAL ORDER (stability of society)

WEALTH (material possessions, money)

\section{PART 6. Positive and Negative Affect Schedule}

This scale consists of a number of words that describe different feelings and emotions. Read each item and then mark the appropriate answer in the space next to that word. Indicate to what extent you have felt this way during the past few weeks. Use the following scale to record your answers.

\begin{tabular}{|c|c|c|c|c|}
\hline 1 & 2 & 3 & 4 & 5 \\
$\begin{array}{c}\text { Very slightly } \\
\text { or not at all }\end{array}$ & A little & Moderately & Quite a bit & Extremely \\
& & & & \\
\hline
\end{tabular}

\begin{tabular}{llllll|l|lllll}
\hline Interested & 1 & 2 & 3 & 4 & 5 & Irritable & 1 & 2 & 3 & 4 & 5 \\
\hline Distressed & 1 & 2 & 3 & 4 & 5 & Alert & 1 & 2 & 3 & 4 & 5 \\
\hline Excited & 1 & 2 & 3 & 4 & 5 & Ashamed & 1 & 2 & 3 & 4 & 5 \\
\hline Upset & 1 & 2 & 3 & 4 & 5 & Inspired & 1 & 2 & 3 & 4 & 5 \\
Strong & 1 & 2 & 3 & 4 & 5 & Nervous & 1 & 2 & 3 & 4 & 5 \\
Guilty & 1 & 2 & 3 & 4 & 5 & Determined & 1 & 2 & 3 & 4 & 5
\end{tabular}




\begin{tabular}{|l|lllll|l|lllll|}
\hline Scared & 1 & 2 & 3 & 4 & 5 & Attentive & 1 & 2 & 3 & 4 & 5 \\
\hline Hostile & 1 & 2 & 3 & 4 & 5 & Jittery & 1 & 2 & 3 & 4 & 5 \\
\hline Enthusiastic & 1 & 2 & 3 & 4 & 5 & Active & 1 & 2 & 3 & 4 & 5 \\
\hline Proud & 1 & 2 & 3 & 4 & 5 & Afraid & 1 & 2 & 3 & 4 & 5
\end{tabular}

\section{PART 7. General questions about the environment (Part 2)}

Please indicate the extent to which you would be willing to engage in the following behaviours:

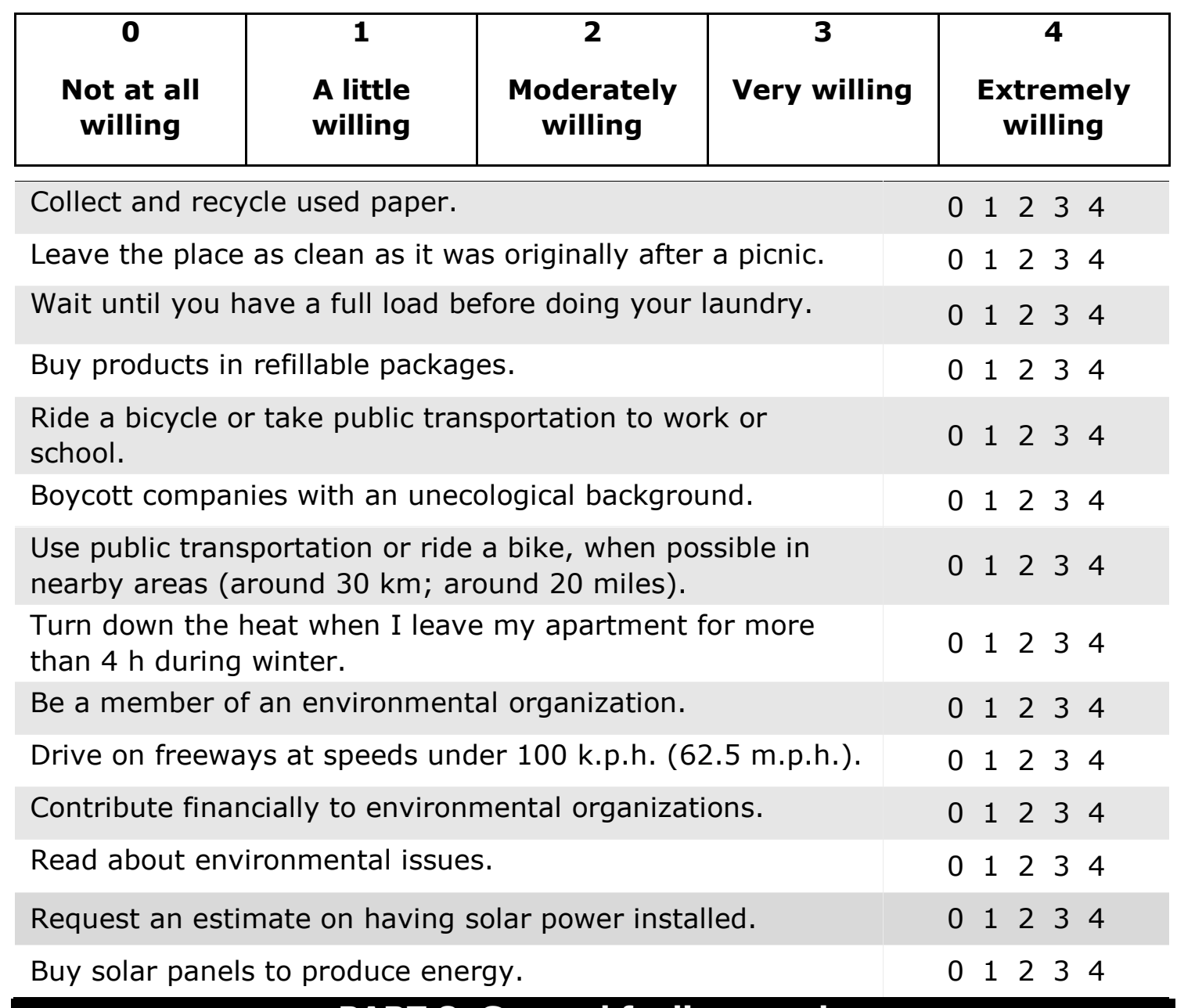

\section{PART 8. General feelings scale}

Below is a list of statements dealing with your general feelings about yourself. If you strongly agree, circle SA. If you agree with the statement, circle A. If you disagree, circle D. If you strongly disagree, circle SD.

\begin{tabular}{|c|c|c|c|}
\hline $\begin{array}{c}\text { SA } \\
\text { Strongly agree }\end{array}$ & $\begin{array}{c}\text { A } \\
\text { Agree }\end{array}$ & $\begin{array}{c}\text { D } \\
\text { Disagree }\end{array}$ & $\begin{array}{c}\text { SD } \\
\text { Strongly disagree }\end{array}$ \\
\hline \multicolumn{3}{|c|}{ On the whole, I am satisfied with myself. } & SA A D SD \\
\hline \multicolumn{3}{|c|}{ At times, I think I am no good at all. } & $S A A D S$ \\
\hline \multicolumn{3}{|c|}{ I feel that I have a number of good qualities. } & $S A$ A D SD \\
\hline
\end{tabular}


I am able to do things as well as most other people.

I feel I do not have much to be proud of.

I certainly feel useless at times.

SA A D SD

I feel that I'm a person of worth, at least on an equal plane with others.

I wish I could have more respect for myself.

SA A D SD

All in all, I am inclined to feel that I am a failure.

SA A D SD

I take a positive attitude toward myself.

SA A D SD

\section{PART 9. Group Identification}

Please select the figure below that best describes the extent to which you feel you are a New Zealander.

\section{How much do you identify with being a New Zealander?}
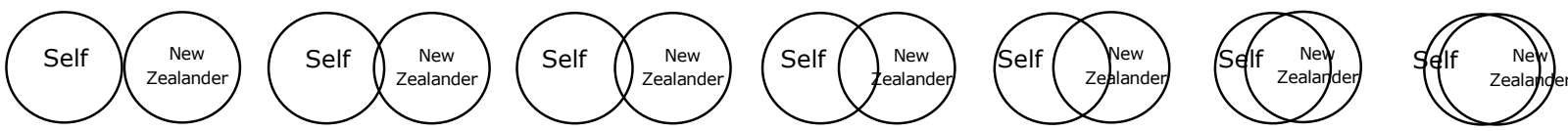

Now, please think about you as a New Zealander and respond to the following statements using the scale below.

\begin{tabular}{|c|c|c|c|c|c|c|}
\hline $\mathbf{1}$ & $\mathbf{2}$ & $\mathbf{3}$ & $\mathbf{4}$ & $\mathbf{5}$ & 6 & $\mathbf{7}$ \\
$\begin{array}{c}\text { Strongly } \\
\text { Disagree }\end{array}$ & Disagree & $\begin{array}{c}\text { Disagree } \\
\text { Somewhat }\end{array}$ & Neutral & $\begin{array}{c}\text { Agree } \\
\text { Somewhat }\end{array}$ & $\begin{array}{c}\text { Agree } \\
\text { Agrongly }\end{array}$ \\
\hline
\end{tabular}

I often regret being a New Zealander.

1223445667

In general, I'm glad to be one of the New Zealand nation.

$\begin{array}{llllllll}1 & 2 & 3 & 4 & 5 & 6 & 7\end{array}$

Overall, I often feel that being a New Zealander is not worthwhile.

$\begin{array}{llllllll}1 & 2 & 3 & 4 & 5 & 6 & 7\end{array}$

I feel good about belonging to the New Zealand nation.

122345567

Overall, New Zealanders are considered good by others.

123345567

Most people consider New Zealanders, on the average, to be more $1 \begin{array}{lllllll}2 & 3 & 4 & 5 & 7\end{array}$ ineffective than other national groups.

In general, others respect New Zealanders.

123345567

In general, others think that New Zealanders are unworthy.

123345567

Overall, being a New Zealander has very little to do with how I feel $1 \begin{array}{lllllll}1 & 2 & 3 & 4 & 5 & 6 & 7\end{array}$ about myself.

Belonging to New Zealand is an important reflection of who I am.

$\begin{array}{llllllll}1 & 2 & 3 & 4 & 5 & 6 & 7\end{array}$

Being a New Zealander is unimportant to my sense of what kind of $\begin{array}{lllllll}1 & 2 & 3 & 4 & 5 & 6 & 7\end{array}$ person I am.

In general, being a New Zealander is an important part of my self $1 \begin{array}{lllllll}1 & 2 & 3 & 4 & 5 & 6 & 7\end{array}$ image. 
You will be debriefed about this experiment shortly. However, I'm curious to know your perception of this study. What do you think the purpose of this study was? Did you have any ideas about what kind of results we might be looking at? What did you think we were hoping to learn about? If you are unsure what the purpose is, please state this:

Please write any general comments you would like to make about this questionnaire or the study in general:

\section{Thank you for your time! :)}




\section{APPENDIX N}

Debriefing Sheet - Experiment 2

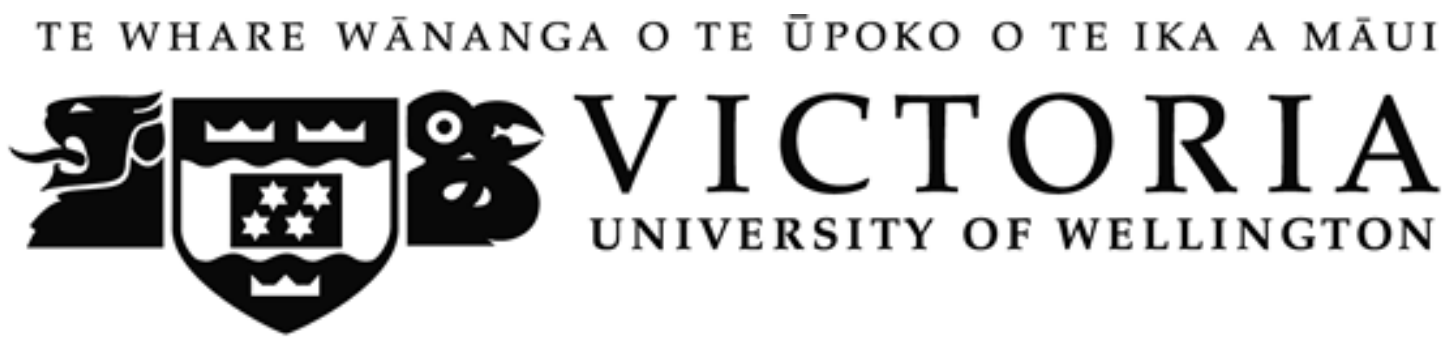

\section{Debriefing statement}

Thank you for participating in this study!

Environmental problems are a result of maladaptive human behaviour. One way to tackle these problems is by fostering values that underlie environmental attitudes and behaviours. This project aims to advance past research by systematically assessing the role of values in motivating individuals' attitudes and behaviours toward the environment.

Past research has shown that there is a strong correspondence between values, attitudes and behavioural intentions. In general, research has shown that self-transcendence values (e.g., social justice, world of peace, loyal, equality, helpful, forgiving, honest, and broad-minded) are positively correlated to environmental attitudes and behaviours. That means people who hold those values are more pro-environmentally friendly. More importantly, research has shown that changes in values can be experimentally manipulated to change behavioural intentions and actual behaviour. I followed this research tradition by using the experimental paradigm developed by Maio et al. (2009) to change behavioural intentions and actual behaviour with the use of values. In their study, Maio et al (2009) tested systematic effects of values, using both manipulation of value change and manipulation of value priming.

My $\mathrm{PhD}$ research will be one of the first endeavours to assess changes in values using an experimental approach while investigating the effect of this on behavioural intentions and actual behaviour towards the environment. My research will contribute to the existing literature by generating more impactful evidence about the relationship between values and environmental behavioural intentions and actual behaviour. The findings will enable strong research-based knowledge that can be used to improve methods to deploy our values in collective decision making.

The present research can also help campaigns that attempt to elicit behavioural change. The findings from my research may offer a more effective solution to current behaviour change programmes that rely almost exclusively on environmental education - a technique that is typically unsuccessful. Finally, it will also include a critical reflection on the way in which campaigns are designed and their impacts on people's values.

A minor deception was used in this research. You were not told that the value ranking or dessert ranking for New Zealanders is fictitious. This deception was necessary because if you knew the rankings were fictitious it might influence your responses. If completing these questionnaires has caused you to feel any distress you can find help at student counselling services at Victoria University of Wellington (http://www.victoria.ac.nz/st_services/counselling/ ; phone number +64 44635310 ).

Thank you again for participating in this research. The research project is being conducted by Pollyane Diniz, Dr. Taciano L. Milfont and Dr. John McClure from the School of Psychology, 
Victoria University of Wellington. The results for this study will be available by the end of the year. If you have any questions regarding your involvement in this research, or issues regarding the research in general, please do not hesitate to contact me via e-mail at Pollyane.Diniz@vuw.ac.nz. 
Information Sheet for Study 3

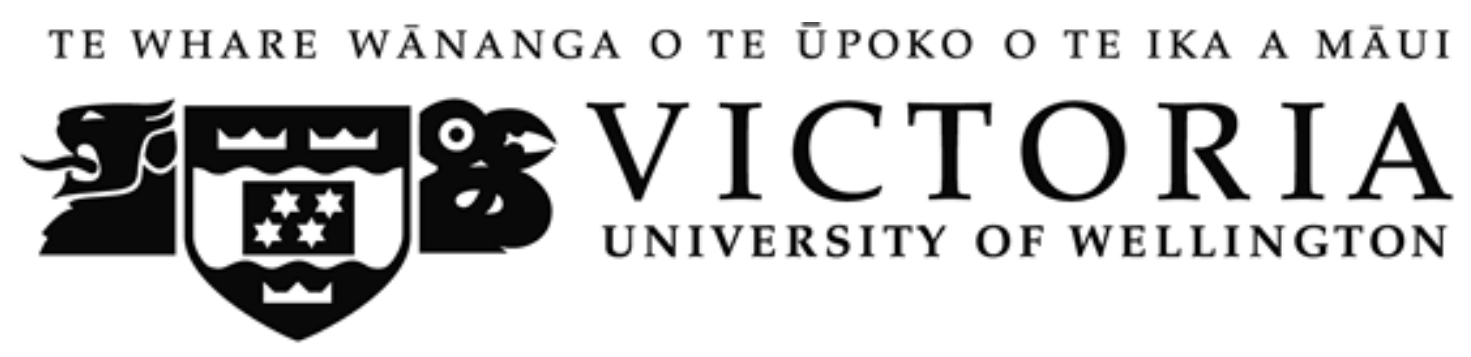

\section{Information Sheet: Data collected for a thesis}

\section{Pollyane Diniz}

\section{Phd Student}

School of Psychology

Victoria University Of

Wellington

Pollyane.Diniz@vuw.ac.nz

Dr. Melanie Vauclair

\section{Research Fellow}

Lisbon University Institute

Melanie.Vauclair@iscte.pt

\section{Dr. Ronald Fischer}

Senior Lecturer

School of Psychology

Victoria University Of

Wellington

Ronald.Fischer@vuw.ac.nz
Dr. John Mcclure

Professor

School of Psychology

Victoria University Of

Wellington

John.Mcclure@vuw.ac.nz
Dr. Taciano L. Milfont

Senior Lecturer

School of Psychology

Victoria University Of

Wellington

Taciano.Milfont@vuw.ac.nz

\section{What is the purpose of this research?}

This research asks about opinions, attitudes, and behaviours on a number of social issues that are relevant to our future. The goal is to understand opinions on these issues.

Who is conducting the research?

Pollyane Diniz is a PhD student. Dr. Fischer, Dr. Milfont and Prof. McClure are supervising this project. This research has been approved by the School of Psychology Human Ethics Committee under delegated authority of Victoria University Wellington's Human Ethics Committee.

\section{What is involved if you agree to participate?}

- If you agree to participate in this study you will be asked to complete a short survey. The survey asks you about your environmental values, specific behaviours, and some demographic questions.

- We anticipate that the survey will take you more than 5 minutes to complete.

- During the research you are free to withdraw, without any penalty, at any point before your survey has been completed.

\section{Privacy and Confidentiality}

- This survey is anonymous. Please do not put your name anywhere on the survey.

- You will never be identified in my research project or in any other presentation or publication. The information you provide will be coded by number only. 
- We will keep your survey for at least five years after publication.

- In accordance with the requirements of some scientific journals and organizations, your coded survey may be shared with other competent researchers.

- Your coded data may be used in other, related studies.

- A copy of the coded data will remain in the custody of Pollyane Diniz in the Environmental Psychology Lab.

\section{What happens to the information that you provide?}

- The data you provide may be used for one or more of the following purposes:

- The overall findings may be submitted for publication in a scientific journal, or presented at scientific conferences.

- The findings will form part of a $\mathrm{PhD}$ thesis that will be submitted for assessment. If you would like to know the results of this study, they will be available approximately next year [2013] upon request via email to Ms. Diniz.

If you have any further questions regarding this study please contact any one of us above.

\section{Statement of consent}

I have read the information about this research and any questions I wanted to ask have been answered to my satisfaction.

I agree to participate in this research. I understand that I can withdraw my consent at any time, without penalty, prior to the end of my participation.

Name:

Signature:

Date:

Student ID:

Copy to:

[a] participant,

[b] researcher (initial both copies below) 


\section{Measures Included in the Online Survey for Study 3}

Note: The survey was administered electronically via a website (www.qualtrix.com). The final layout and item order was only determined when the questions were loaded and setup online. The survey on the following pages gives an indication of the presentation and a list of the items to be used.

\section{General Ecological Behaviour (GEB) Scale}

INSTRUCTION: Please indicate the extent to which you would be willing to engage in the following behaviours:

\begin{tabular}{|c|c|c|c|c|}
\hline $\begin{array}{c}\text { Not at all } \\
\text { willing }\end{array}$ & $\begin{array}{c}1 \\
\text { A little } \\
\text { willing }\end{array}$ & $\begin{array}{c}\text { Moderately } \\
\text { willing }\end{array}$ & Very willing & $\begin{array}{c}\mathbf{4} \\
\text { Extremely } \\
\text { willing }\end{array}$ \\
\hline
\end{tabular}

$\underline{\text { Items }}$

\section{Energy conservation}

Own energy efficient household devices

Wait until I have a full load before doing my laundry

Wash dirty clothes without prewashing

In hotels, I have the towels changed daily

Use a clothes dryer

Buy solar panels to produce energy

Use renewable energy sources

In the winter, keep the heat on so that I do not have to wear a sweater

In the winter, leave the windows open for long periods of time to let in fresh air

In winter, turn down the heat when I leave my apartment for more than $4 \mathrm{~h}$

Prefer to shower rather than to take a bath

\section{Mobility and transportation}

Drive my car in or into the city

Drive on freeways at speeds under $100 \mathrm{kph}(1 / 462.5 \mathrm{mph})$

Keep the engine running while waiting in front of a railroad crossing or in a traffic jam 
At red traffic lights, keep the engine running

Drive to where I want to start my hikes

Refrain from owning a car

Be a member of a carpool

Drive in such a way as to keep my fuel consumption as low as possible

Own a fuel-efficient automobile (less than 71 per $100 \mathrm{~km}$; i.e., less than 3 gallons per 100 miles)

For longer journeys (more than $6 \mathrm{~h}$ ), take an airplane

In nearby areas (around $30 \mathrm{~km}$; around 20 miles), use public transportation or ride a bike

Ride a bicycle or take public transportation to work or school

\section{Waste avoidance}

Buy milk in returnable bottles

Take a plastic bag if it is offered in a store.

Reuse my shopping bags

Buy beverages in cans

Buy products in refillable packages

\section{Consumerism}

Use fabric softener with my laundry

Use an oven cleaning spray to clean my oven

Kill insects with a chemical insecticide

Use a chemical air freshener in my bathroom

Buy convenience foods

Buy seasonal produce

Buy bleached and colored toilet paper

Buy meat and produce with eco-labels

Buy domestically grown wooden furniture

\section{Recycling}

Collect and recycle used paper

Bring empty bottles to a recycling bin

Put dead batteries in the garbage 
After meals, dispose of leftovers in the toilet

Vicarious, social behaviours toward conservation

After a picnic, leave the place as clean as it was originally

Be a member of an environmental organization

Read about environmental issues

Contribute financially to environmental organizations

Talk with friends about problems related to the environment

Have pointed out unecological behaviour to someone

Boycott companies with an unecological background

Have already looked into the pros and cons of having a private source of solar power

Requested an estimate on having solar power installed 


\section{Portrait Values Questionnaire - PVQ}

\section{PVQ-R2 Male}

INSTRUCTION: Here we briefly describe different people. Please read each description and think about how much that person is or is not like you. Put an X in the box to the right that shows how much the person described is like you.

\begin{tabular}{|c|c|c|c|c|}
\hline & & & & \\
\hline $\begin{array}{l}\text { Jot like } \\
\text { me at } \\
\text { all }\end{array}$ & $\begin{array}{l}\text { Not like } \\
\text { me }\end{array}$ & $\begin{array}{l}\text { A li } \\
\text { like }\end{array}$ & $\begin{array}{l}\text { Moder- } \\
\text { ately } \\
\text { like me }\end{array}$ & $\begin{array}{c}\text { Like } \\
\text { me }\end{array}$ \\
\hline
\end{tabular}

1. It is important to him to form his own understanding of things

2. It is important to him that there is stability and order in the wider society .

3. It is important to him to have a good time.

4. It is important to him to avoid upsetting other people.

5. It is important to him to protect the weak and vulnerable people in society.

6. It is important to him that people do what he says they should.

7. It is important to him never to be boastful or selfimportant.

8. It is important to him to care for nature.

9. It is important to him that no one should ever shame him.

10. It is important to him always to look for different things to do.

11. It is important to him to take care of people he is close to.

12. It is important to him to have the money to do whatever he wants.

13. It is very important to him to avoid disease and protect his health.

14. It is important to him to be tolerant toward all kinds of people and groups.

15. It is important to him never to violate rules or regulations.

16. It is important to him to make his own decisions about his life.

17. It is important to him to have ambitions in life.

18. It is important to him to maintain traditional values and ways of thinking. 


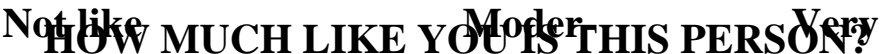 \\ me at Not like A little ately Like much all me like me like me me like me}

19. It is important to him that people he knows have full confidence in him.

20. It is important to him to be wealthy.

21. It is important to him to take part in activities to defend nature.

22. It is important to him never to annoy anyone.

23. It is important to him to have his own original ideas.

24. It is important to him to protect his public image.

25. It is very important to him to help the people dear to him.

26. It is important to him to be personally safe and secure.

27. It is important to him to be a dependable and trustworthy friend.

28. It is important to him to take risks that make life exciting.

29. It is important to him to have the power to make people do what he wants..

30. It is important to him to plan his activities independently.

31. It is important to him to follow rules even when noone is watching.

32. It is important to him to be very successful.

33. It is important to him to follow his family's customs or the customs of a religion.

34. It is important to him to listen to and understand people who are different from him.

35. It is important to him to have a strong state that can defend its citizens.

36. It is important to him to enjoy life's pleasures.

37. It is important to him that every person in the world have equal opportunities in life.

38. It is important to him to be humble.

39. It is important to him always to keep learning.

40. It is important to him to honor the traditional practices of his culture.

41. It is important to him to be the one who tells others what to do..

42. It is important to him to obey all the laws. 


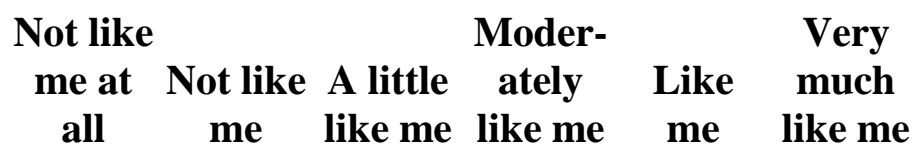

43. It is important to him to have all sorts of new experiences..

44. It is important to him to own expensive things that show his wealth

45. It is important to him to protect the natural environment from destruction or pollution.

46. It is important to him to take advantage of every opportunity to have fun.

47. It is important to him to concern himself with every need of his dear ones.

48. It is important to him that people recognise what he achieves.

49. It is important to him never to be humiliated.

50. It is important to him that his country protect itself against all threats.

51. It is important to him never to make other people angry.

52. It is important to him that everyone be treated justly, even people he doesn't know.

53. It is important to him never to do anything dangerous.

54. It is important to him never to seek public attention or praise.

55. It is important to him that all his friends and family can rely on him completely.

56. It is important to him to be free to choose what he does by himself.

57. It is important to him to accept people even when he disagrees with them. 


\section{PVQ-R2 Female}

INSTRUCTION: Here we briefly describe different people. Please read each description and think about how much that person is or is not like you. Put an X in the box to the right that shows how much the person described is like you.

HOW MUCH LIKE YOU IS THIS PERSON?

Not like

Moder-

me at Not like A little ately Like

Very HO all me like me like me me like me

1. It is important to her to develop her own understanding of things.

2. It is important to her that there is stability and order in the wider society .

3. It is important to her to have a good time.

4. It is important to her to avoid upsetting other people.

5. It is important to her to protect the weak and vulnerable people in society.

6. It is important to her that people do what she says they should.

7. It is important to her never to be boastful or selfimportant.

8. It is important to her to care for nature.

9. It is important to her that no one should ever shame her.

10. It is important to her always to look for different things to do.

11. It is important to her to take care of people she is close to.

12. It is important to her to have the money to do whatever she wants.

13. It is very important to her to avoid disease and protect her health.

14. It is important to her to be tolerant toward all kinds of people and groups.

15. It is important to her never to violate rules or regulations.

16. It is important to her to make her own decisions about her life.

17. It is important to her to have ambitions in life.

18. It is important to her to maintain traditional values and ways of thinking.

19. It is important to her that people she knows have full confidence in her. 
HOW MUCH LIKE YOU IS THIS PERSON?

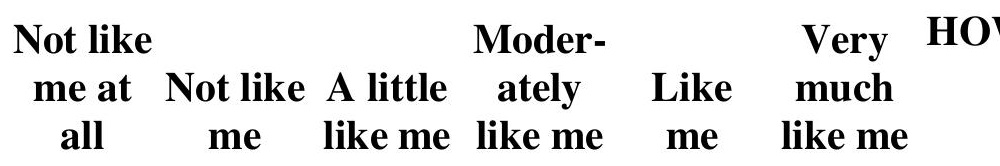

20. It is important to her to be wealthy.

21. It is important to her to take part in activities to defend nature.

22. It is important to her never to annoy anyone.

23. It is important to her to have her own original ideas.

24. It is important to her to protect her public image.

25 . It is very important to her to help the people dear to her.

26. It is important to her to be personally safe and secure.

27. It is important to her to be a dependable and trustworthy friend.

28. It is important to her to take risks that make life exciting.

29. It is important to her to have the power to make people do what she wants..

30. It is important to her to plan her activities independently.

31. It is important to her to follow rules even when noone is watching.

32. It is important to her to be very successful.

33. It is important to her to follow her family's customs or the customs of a religion.

34. It is important to her to listen to and understand people who are different from her.

35. It is important to her to have a strong state that can defend its citizens.

36. It is important to her to enjoy life's pleasures.

37. It is important to her that every person in the world have equal opportunities in life.

38. It is important to her to be humble.

39. It is important to her always to keep learning.

40. It is important to her to honor the traditional practices of her culture.

41. It is important to her to be the one who tells others what to do..

42. It is important to her to obey all the laws.

43. It is important to her to have all sorts of new experiences.. 
HOW MUCH LIKE YOU IS THIS PERSON?

Not like

Moder-

Very

HO

me at Not like A little ately Like much

all me like me like me me like me

44. It is important to her to own expensive things that show her wealth

45. It is important to her to protect the natural environment from destruction or pollution.

46. It is important to her to take advantage of every opportunity to have fun.

47. It is important to her to concern herself with every need of her dear ones.

48. It is important to her that people recognise what she achieves.

49. It is important to her never to be humiliated.

50. It is important to her that her country protect itself against all threats.

51. It is important to her never to make other people angry.

52. It is important to her that everyone be treated justly, even people she doesn't know.

53. It is important to her never to do anything dangerous.

54. It is important to her never to seek public attention or praise.

55. It is important to her that all her friends and family can rely on her completely.

56. It is important to her to be free to choose what she does by herself..

57. It is important to her to accept people even when she disagrees with them. 


\section{Self-control measure}

INSTRUCTION: Please indicate the extent to which each affirmation below describes you:

Not at all

1

2

Partially

Completely

4

5

6

7

I am good at resisting temptation.

I have a hard time breaking bad habits.

I am lazy.

I say inappropriate things.

I do certain things that are bad for me, if they are fun.

I refuse things that are bad for me.

I wish I had more self-discipline.

People would say that I have iron self-discipline.

Pleasure and fun sometimes keep me from getting work done.

I have trouble concentrating.

I am able to work effectively toward long term goals.

Sometimes I can't stop myself from doing something, even if I know it is wrong.

I often act without thinking through all the alternatives. 


\section{Self-Efficacy measure}

INSTRUCTION: Please indicate the extent to which each affirmation below describes you.

\begin{tabular}{|c|c|c|c|}
\hline Not at all true & Hardly true & Moderately true & Exactly true \\
\hline
\end{tabular}

I can always manage to solve difficult problems if I try hard enough.

If someone opposes me, I can find the means and ways to get what I want.

It is easy for me to stick to my aims and accomplish my goals.

I am confident that I could deal efficiently with unexpected events.

Thanks to my resourcefulness, I know how to handle unforeseen situations.

I can solve most problems if I invest the necessary effort.

I can remain calm when facing difficulties because I can rely on my coping abilities.

When I am confronted with a problem, I can usually find several solutions.

If I am in trouble, I can usually think of a solution.

I can usually handle whatever comes my way. 


\section{Moral identity scale}

INSTRUCTION: Listed below are some characteristics that might describe a person:

Caring, Compassionate, Fair, Friendly, Generous, Helpful, Hardworking, Honest, and Kind

The person with these characteristics could be you or it could be someone else. For a moment, visualise in your mind the kind of person who has these characteristics.

Imagine how that person would think, feel, and act. When you have a clear image of what this person would be like, please indicate to what extent you agree with the following statements by using the following rating scale ranging from 'strongly disagree' to 'strongly agree'.

Strongly disagree

Undecided

Strongly agree

1

2

3

4

5

6

7

It would make me feel good to be a person who has these characteristics.

Being someone who has these characteristics is an important part of who I am.

I often wear clothes that identify me as having these characteristics.

I would be ashamed to be a person who had these characteristics.

The types of things I do in my spare time (e.g., hobbies) clearly identify me as having these characteristics.

The kinds of books and magazines that I read identify me as having these characteristics.

Having these characteristics is not really important to me.

The fact that I have these characteristics is communicated to others by my membership in certain organizations.

I am actively involved in activities that communicate to others that I have these characteristics.

I strongly desire to have these characteristics. 


\section{Consideration of Future Consequences - CFC Scale}

Please read each of the following statements and, as honestly as you can, answer the following question: How characteristic or true is this of you?

\begin{tabular}{|c|c|c|c|c|c|c|c|c|c|}
\hline $\begin{array}{c}1 \\
\text { Very } \\
\text { uncharacteristic }\end{array}$ & $\begin{array}{c}2 \\
\text { Uncharacteristic }\end{array}$ & $\begin{array}{c}3 \\
\text { Neutral }\end{array}$ & $\begin{array}{l}\quad 4 \\
\text { Characteristic }\end{array}$ & \multicolumn{6}{|c|}{$\begin{array}{c}5 \\
\text { Very characteristic }\end{array}$} \\
\hline \multicolumn{4}{|c|}{$\begin{array}{l}\text { 1. I consider how things might be in the future, and try to influence } \\
\text { those things with my day to day behaviour. }\end{array}$} & 1 & 2 & 3 & & & 5 \\
\hline \multicolumn{4}{|c|}{$\begin{array}{l}\text { 2. Often I engage in a particular behaviour in order to achieve } \\
\text { outcomes that may not result for many years. }\end{array}$} & 1 & 2 & 3 & & 4 & 5 \\
\hline \multicolumn{4}{|c|}{$\begin{array}{l}\text { 3. I only act to satisfy immediate concerns, figuring the future will } \\
\text { take care of itself. }\end{array}$} & 1 & 2 & 3 & & 4 & 5 \\
\hline \multicolumn{4}{|c|}{$\begin{array}{l}\text { 4. My behaviour is only influenced by the immediate (i.e., a matter of } \\
\text { days or weeks) outcomes of my actions. }\end{array}$} & 1 & 2 & 3 & & 4 & 5 \\
\hline \multicolumn{4}{|c|}{$\begin{array}{l}\text { 5. My convenience is a big factor in the decisions I make or the } \\
\text { actions I take. }\end{array}$} & 1 & 2 & 3 & & 4 & 5 \\
\hline \multicolumn{4}{|c|}{$\begin{array}{l}\text { 6. I am willing to sacrifice my immediate happiness or well-being in } \\
\text { order to achieve future outcomes. }\end{array}$} & 1 & 2 & 3 & & 4 & 5 \\
\hline \multicolumn{4}{|c|}{$\begin{array}{l}\text { 7. I think it is important to take warnings about negative outcomes } \\
\text { seriously even if the negative outcome will not occur for many years. }\end{array}$} & 1 & 2 & 3 & 4 & 4 & 5 \\
\hline \multicolumn{4}{|c|}{$\begin{array}{l}\text { 8. I think it is more important to perform a behaviour with important } \\
\text { distant consequences than a behaviour with less-important immediate } \\
\text { consequences. }\end{array}$} & 1 & 2 & 3 & & + & \\
\hline \multicolumn{4}{|c|}{$\begin{array}{l}\text { 9. I generally ignore warnings about possible future problems because } \\
\text { I think the problems will be resolved before they reach crisis level. }\end{array}$} & 1 & 2 & 3 & & 4 & 5 \\
\hline \multicolumn{4}{|c|}{$\begin{array}{l}\text { 10. I think that sacrificing now is usually unnecessary since future } \\
\text { outcomes can be dealt with at a later time. }\end{array}$} & 1 & 2 & 3 & & 4 & 5 \\
\hline \multicolumn{4}{|c|}{$\begin{array}{l}\text { 11. I only act to satisfy immediate concerns, figuring that I will take } \\
\text { care of future problems that may occur at a later date. }\end{array}$} & 1 & 2 & 3 & & 4 & 5 \\
\hline \multicolumn{4}{|c|}{$\begin{array}{l}\text { 12. Since my day to day work has specific outcomes, it is more } \\
\text { important to me than behaviour that has distant outcomes. }\end{array}$} & 1 & 2 & 3 & & + & 5 \\
\hline
\end{tabular}




\section{$\underline{\text { Social Demographic questions }}$}

\section{Background questions}

(Please remember that your responses are confidential)

\begin{tabular}{|c|c|c|c|}
\hline \multirow{3}{*}{$\begin{array}{l}\text { 1. How old are you? } \\
\text { years. }\end{array}$} & \multicolumn{2}{|c|}{ 2. What is your gender? } & \multirow{2}{*}{$\begin{array}{l}\text { 3. Are you a member of any } \\
\text { environmental organisation } \\
\text { (e.g., Greenpeace)? }\end{array}$} \\
\hline & 1. Femal & 2. Male & \\
\hline & & & 1. Yes \\
\hline
\end{tabular}

4. Were you born in New Zealand?

2. No

5. Which ethnic group do you belong to? Please indicate the group you most strongly identify with.
1. New Zealand European (Pākehā)
4. Māori
2. Pacific Nations
5. Asian
3. Indian
6. Other (please specify):

\section{Thank you for your time! (:)}




\title{
APPENDIX Q
}

\author{
Immediate Debriefing for Study 3
}

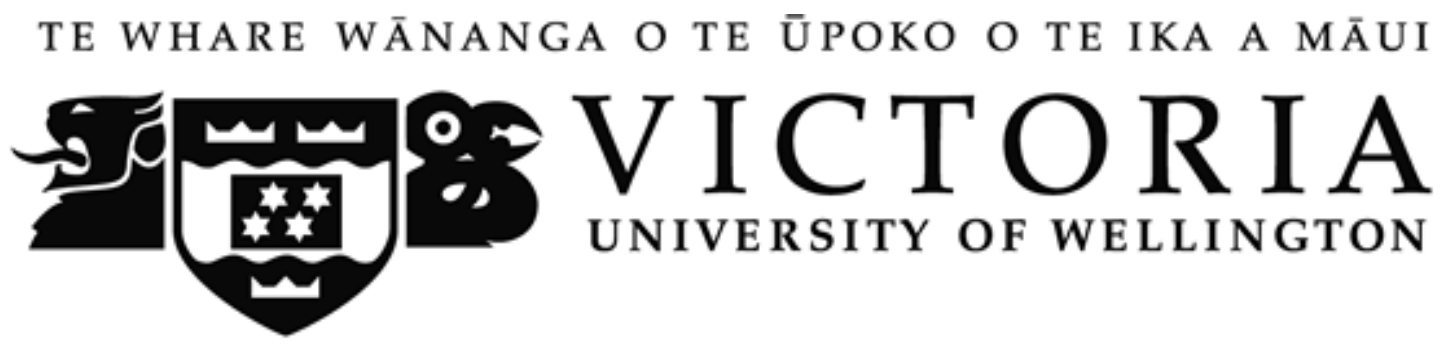

\section{Debriefing statement: Immediate debriefing.}

Thank you for participating in this study.

Environmental problems are a result of maladaptive human behaviour. One way to tackle these problems is to foster values that underlie environmental attitudes and behaviours. This project aims to advance past research by assessing the role of values in individuals' attitudes and behaviours toward the environment.

A number of recent studies have examined the links between values and environmental engagement. This research has shown that there is a strong correspondence between values, attitudes and behavioural intentions, and that self-transcendence values (e.g., social justice and world of peace) are positively related to environmental attitudes and behaviours. So people who hold those values are more environmentally friendly. However, this relationship may be moderated by individual characteristics such as selfcontrol, self-efficacy, moral identity and consideration of future consequences. The main goal of this study was test how these characteristics strength or weaken the relation between values and environmental past behaviour.

My research will contribute to the literature by generating more impactful evidence about the relationship between values and environmental behavioural intentions and actual behaviour. The findings will provide research-based knowledge that can be used to improve ways to activate our values in decision making.

This research may also help campaigns that attempt to generate behavioural change. The findings may offer a more effective solution than current programmes that rely almost exclusively on environmental education - a technique that is typically unsuccessful. Finally, it will also include a critical reflection on the way in which campaigns are designed and their impacts on people's values.

Thank you again for participating in this research. The research project is being conducted by Pollyane Diniz, Dr. Ronald Fischer, Professor John McClure, Dr. Taciano L. Milfont from the School of Psychology, Victoria University of Wellington, and Melanie Vauclair from Lisbon University Institute. If completing these questionnaires has caused you to feel any distress you can find help at student counselling services at Victoria University of Wellington (http://www.victoria.ac.nz/st_services/counselling/ ; phone number +64 44635310 ). If you have any questions regarding your involvement 
in this research, or issues regarding the research in general, please do not hesitate to contact me via e-mail at Pollyane.Diniz@vuw.ac.nz. 